\title{
Augmented Propeller Design with the use of a Passive Circulation Control Pressurization System
}

Jonathan Kweder

West Virginia University

Follow this and additional works at: https://researchrepository.wvu.edu/etd

\section{Recommended Citation}

Kweder, Jonathan, "Augmented Propeller Design with the use of a Passive Circulation Control Pressurization System" (2012). Graduate Theses, Dissertations, and Problem Reports. 444.

https://researchrepository.wvu.edu/etd/444

This Dissertation is protected by copyright and/or related rights. It has been brought to you by the The Research Repository @ WVU with permission from the rights-holder(s). You are free to use this Dissertation in any way that is permitted by the copyright and related rights legislation that applies to your use. For other uses you must obtain permission from the rights-holder(s) directly, unless additional rights are indicated by a Creative Commons license in the record and/ or on the work itself. This Dissertation has been accepted for inclusion in WVU Graduate Theses, Dissertations, and Problem Reports collection by an authorized administrator of The Research Repository @ WVU.

For more information, please contact researchrepository@mail.wvu.edu. 


\title{
Augmented Propeller Design with the use of a Passive Circulation Control Pressurization System
}

Submitted by: Jonathan Kweder

Dissertation Submitted to the

Benjamin M. Statler College of Engineering and Mineral Resources

at West Virginia University

in Partial Fulfillment for the Degree of

Doctorate of Philosophy in Mechanical Engineering

\author{
Submitted to: \\ James E. Smith, Ph.D., Chairperson \\ MaryAnn Clarke, Ph.D. \\ Kenneth H. Means, Ph.D. \\ Andrew C. Nix, Ph.D. \\ William S. Wayne, Ph.D.
}

Department of Mechanical and Aerospace Engineering

Morgantown, West Virginia

2012

Keywords: Circulation Control, Propeller, Passive Pressurization Copyright 2012 Jonathan Kweder 


\section{Abstract \\ Augmented Propeller Design with the use of a Passive Circulation Control \\ Pressurization System}

\section{Jonathan Kweder}

Circulation control is a high-lift device used on the main wing of an aircraft. This technology has been in the research and development phase for over sixty years primarily for fixed-wing aircraft when the early models were referred to as "blown flaps." Circulation control works by increasing the near surface velocity of the airflow over the leading edge and/or trailing edge of a specially designed aircraft wing using a series of blowing slots that eject high velocity jets of air. The wing has a rounded trailing edge, and ejects the air tangentially, through these slots inducing the Coandă Effect. This phenomenon keeps the boundary layer jet attached to the wing surface longer than a conventional wing and thus increases the lift generated on the wing surface due to the relaxation of the Kutta Condition for the rounded trailing edge. The circulation control airflow adds to the lift force through conventional airfoil lift production, by altering the circulation of stream lines around the airfoil.

The main purpose of the circulation control for fixed wing aircraft is to increase the lifting force when large lifting forces and/or slow speeds are required, such as at take-off and landing. Wing flaps and slats are currently used during landing on almost all fixed wing aircraft and on take-off by larger jets. While flaps and slats are effective in increasing lift, they do so with a penalty of increased drag. The benefit of the circulation control wing is that no extra drag is created from the movement of surfaces into the airflow around the wing and the lift coefficient is greatly increased.

Taking advantage of circulation control generally includes the addition of extra weight to an aircraft or siphoning some of the power off the engine to run the circulation control hardware. It was the goal of this research to find an alternative way of pumping circulation control pressurization air to the trailing edge slot located on an unmanned aerial vehicle propeller. The design called for the rerouting of stagnation pressure on the frontal propeller area, through the inside of the propeller blades, to an ejection slot on the training edge of the propeller blade. This allowed for the forward velocity of the aircraft to drive the pressurization of the circulation control plenum passively, or without additional hardware.

For this study, a Clark-Y airfoil section propeller with an overall diameter of 24 inches was designed and tested in both a West Virginia University wind tunnel and the vertical wind tunnel at Wright-Patterson Air Force Base as well as computationally through the use of Fluent software and Blade Element Analysis methods. The comparison of both the augmented propeller and the unaugmented (baseline) propeller showed a 5.74 percent increase in efficiency by using the circulation control augmentation. This increase in efficiency is shown to act over the entire range of flight envelopes of the aircraft. It is shown to be particularly beneficial at advance ratios above 0.30 , normal operating conditions of propeller driven $U A V$ 's. 


\section{Table of Contents}

ABSTRACT ........................................................................................................................ II

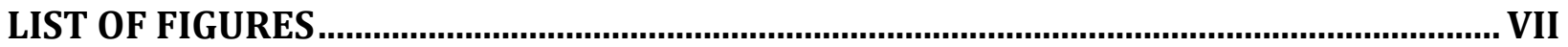

LIST OF TABLES..........................................................................................................XV

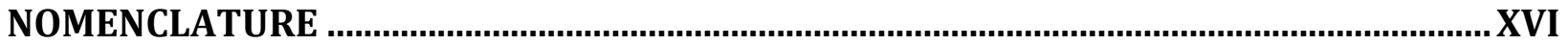

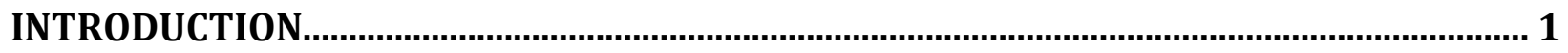

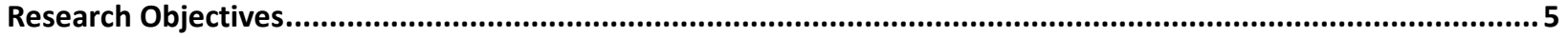

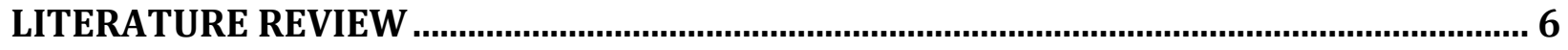

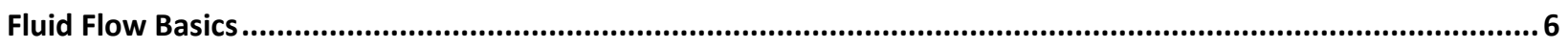

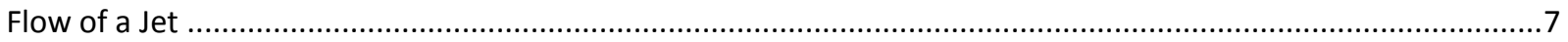

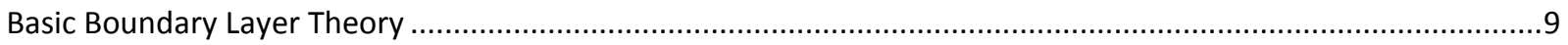

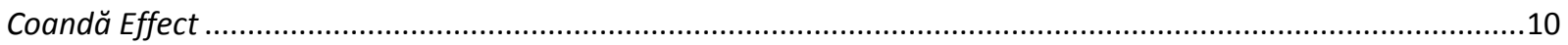

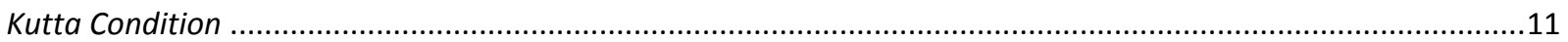

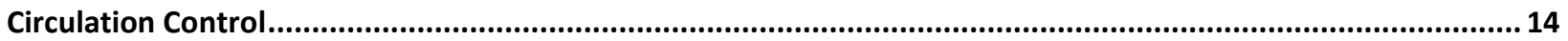

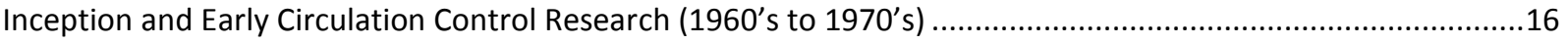

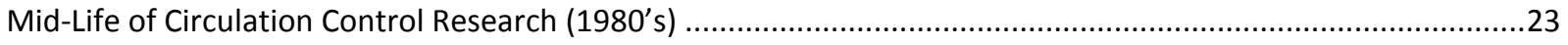

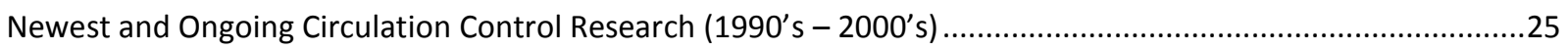

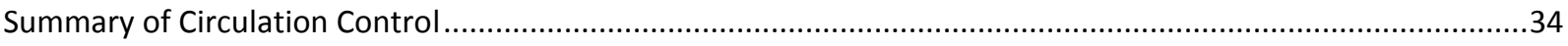

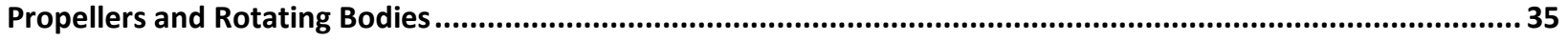

Experimental Testing Facilities................................................................................................47

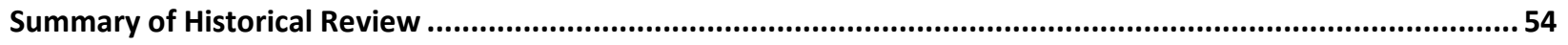

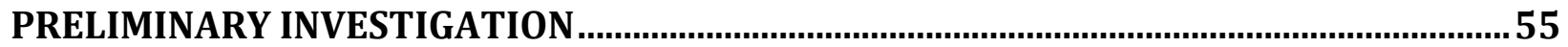

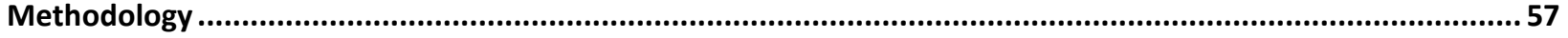

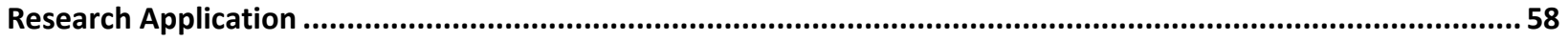

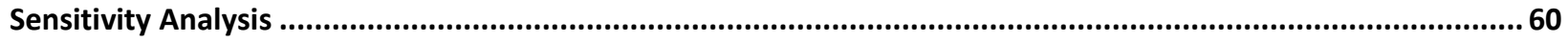

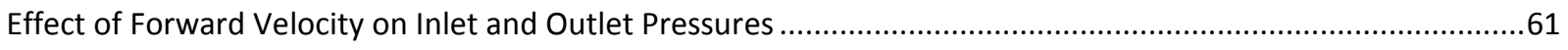

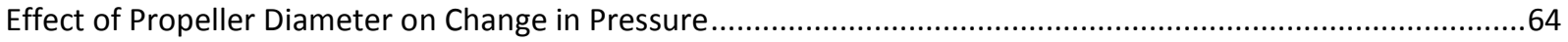




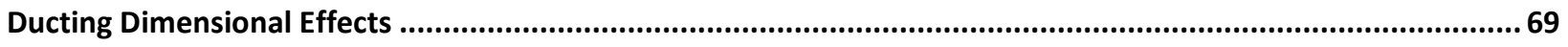

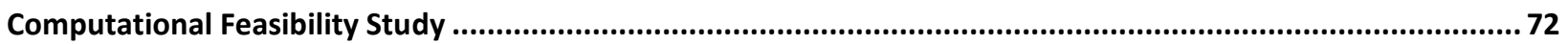

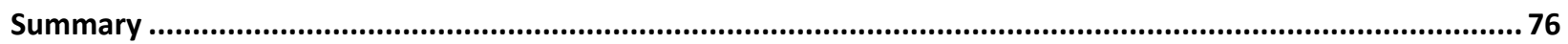

PROPELLER DESIGN ................................................................................................................ 78

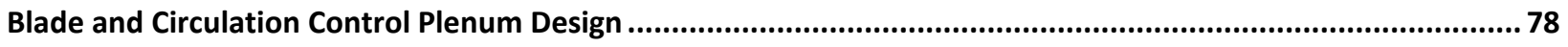

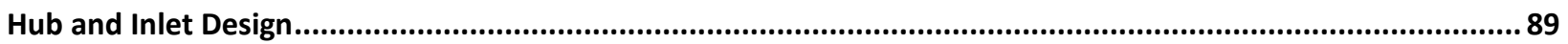

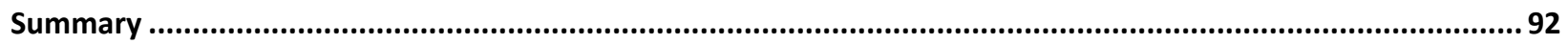

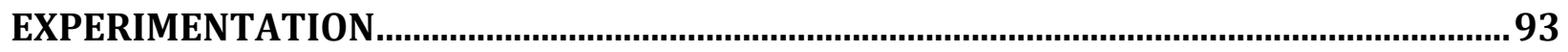

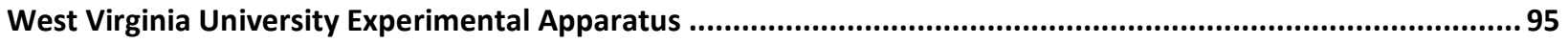

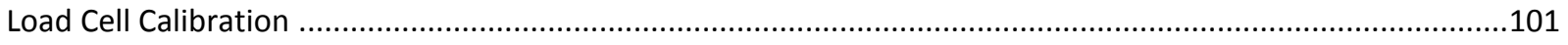

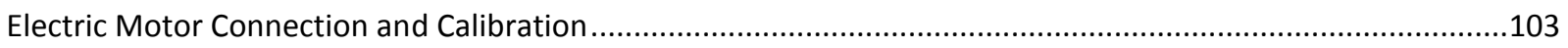

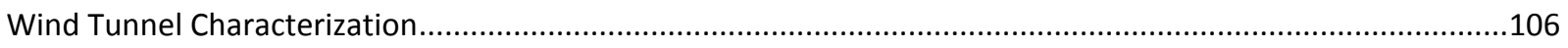

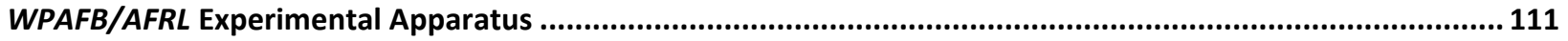

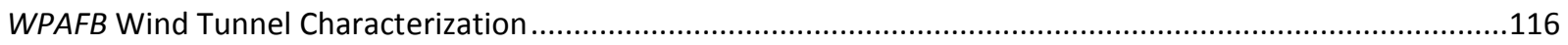

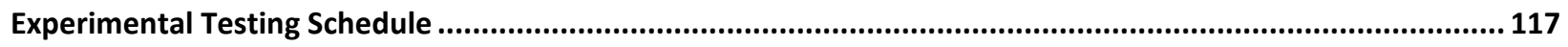

Computational Modeling Design and Evaluation ..................................................................................... 123

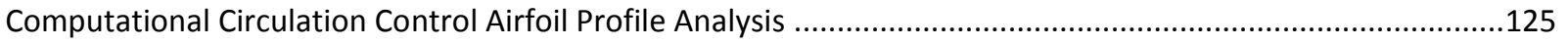

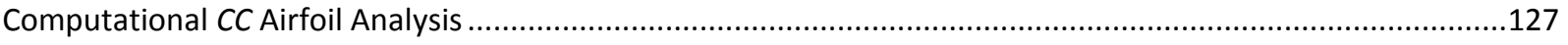

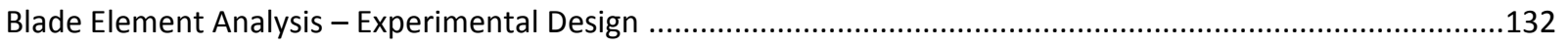

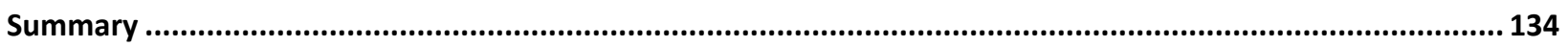

EXPERIMENTAL, COMPUTATIONAL, AND ANALYTICAL RESULTS ............................... 135

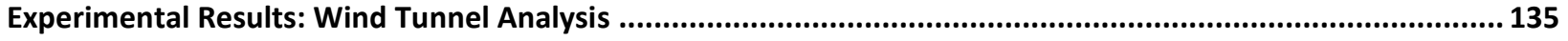

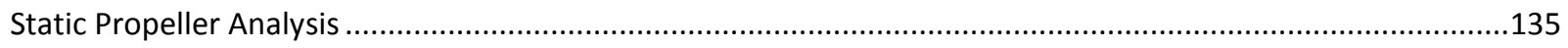

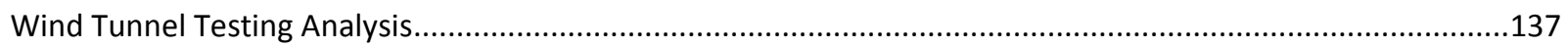

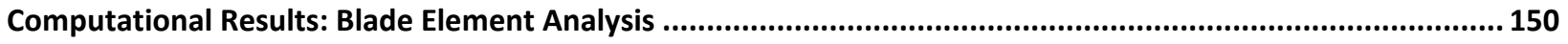

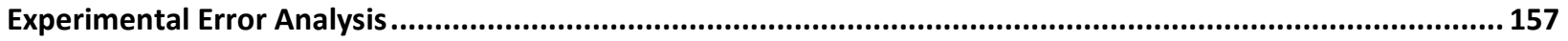


REFERENCES

ACKNOWLEDGEMENTS

APPENDIX A - CINCINNATI SQAF - 270 SPECIFICATIONS

APPENDIX B - RAPID PROTOTYPING MACHINE SPECIFICATIONS

182

APPENDIX C - THREE-DIMENSIONAL SCANNING CAMERA

APPENDIX D - ROTATIONAL STUDY OF AUGMENTED PROPELLER 186

APPENDIX E - BRUSHLESS MOTOR SPECIFICATIONS 190

APPENDIX F - ELECTRONIC SPEED CONTROLLER SPECIFICATIONS 191

APPENDIX G - CLARK-Y SIMULATED AIRFOIL DATA 196

Angle-of-Attack Sweep at $R E=90,000$ 196

Angle-of-Attack Sweep at $R E=900,000$ 200

APPENDIX H - TWO-DIMENSIONAL CLARK-Y AIRFOIL DATA ….................................. 203

APPENDIX I - EXPERIMENTAL THRUST COMPARISONS 205

WVU Experiments. 205

WPAFB Experiments 206

APPENDIX J - EXPERIMENTAL TORQUE COMPARISONS 208

WVU Experiments. 208 210 
APPENDIX L - CASTLE LINK SPEED CONTROLLER DATA LOGGER ................................ 214

Logged Data from WPAFB Experiment - Unaugmented Propeller .......................................................214

Logged Data from WPAFB Experiment - Augmented Propeller............................................................. 217

APPENDIX M - ADDITIONAL COMPUTATIONAL CFD IMAGES ..................................... 219

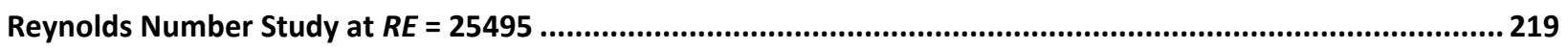

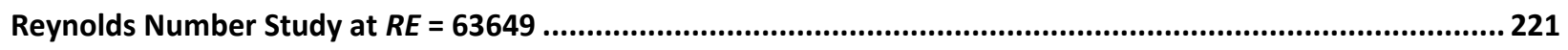

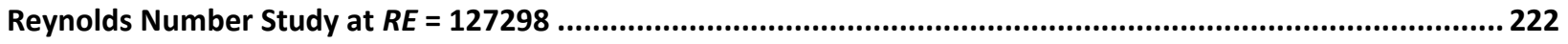




\section{List of Figures}

Figure 1. Effect of a Wall Jet on Boundary Layer (Meyer, 1972) ................................................ 8

Figure 2. Flow of a Two-Dimensional Jet Around a Circular Cylinder (Newman, 1961) ........... 11

Figure 3. Potential Rear Stagnation Locations over Airfoils with Sharp Trailing Edges (Katz, 2001) 13

Figure 4. Typical Circulation Control Airfoil Lift Capability per Momentum Coefficient (Englar, 1973) 20

Figure 5. Reverse Blowing Circulation Controlled Rotor Hub (Reader, 1977) ............................ 22

Figure 6. Concept Drawing of $C C$ Controlled Tractor Trailer (Englar, 2006) ............................. 28

Figure 7. Circulation Control Tractor Trailier being Road-Tested (Englar, 2006) ....................... 29

Figure 8. CC Wind Turbine Blade Planfom Dimensions (Tongchitpakdee, 2007) ...................... 32

Figure 9. Circulation Control Plenum and Rib Spacing of CCHB Model (Angle, 2008) ............ 33

Figure 10. Vortex Model Velocity Field with Vortices and Blade Positions (Wilhelm, et al., 2009) 34

Figure 11. Leonardo da Vinci's Helicopter Design (Vezzosi, 1997) ............................................. 35

Figure 12. Different Propeller Planforms (Dommasch, Sherby, and Connolly, 1961) ................. 38

Figure 13. Physical Representation of Disk Actuator Theory (Nelson, 1944 ) ............................. 40

Figure 14. Generic Rotational Path of a Propeller in Two Dimensions (Dommasch, et. al, 1961)

Figure 15. Blade Element Angular Relationships (Nelson, 1944)............................................. 42

Figure 16. How the Wright Brother's Propeller was Cut from Three Planks (Ash, 2003) ........... 43

Figure 17. Propeller Manufacturing Techniques (Nelson, 1944) ………………………........... 43

Figure 18. Slipstream of a Generic Propeller (Nelson, 1944).................................................... 44 
Figure 19. Force Breakdown on a Conventional Four-Bladed Propeller (Stepniewski, 1984) .... 45

Figure 20. Wind Tunnel Propeller Test Stand (National Aerospace Laboratory) ...................... 48

Figure 21. Water Tunnel Propeller Test Stand (Aria Wind Power Systems Inc.) ...................... 49

Figure 22. WPAFB-AFRL Vertical Wind Tunnel .......................................................... 50

Figure 23. Cross Sectional View of WPAFB Vertical Wind Tunnel..................................... 52

Figure 24. Side View of General CC Propeller Blade (Not to scale) ..................................... 56

Figure 25. Top View of General CC Propeller Blade (Not to scale) ....................................... 57

Figure 26. Trend Study of Forward Velocity Effect on Circulation Control Inlet Pressure

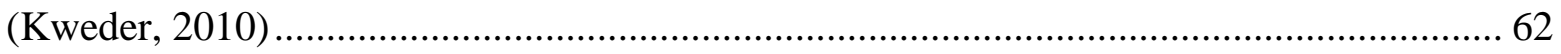

Figure 27. Effect of Forward Speed on Plenum Pressure (Kweder, 2010).............................. 63

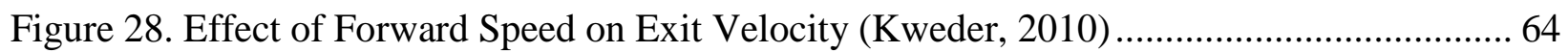

Figure 29. Change in Pressure as it Relates to Varying Propeller Diameter (Kweder, 2010) ...... 66

Figure 30. Plenum Pressure versus Blade Diameter Trend Study as a Function of Propeller

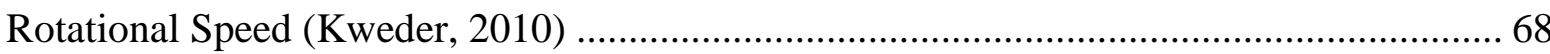

Figure 31. Effect of Change in Plenum Cross-sectional Area on Outlet Pressure (Kweder, 2010)

Figure 32. Effect of Interior Duct Length on Outlet Pressure (Kweder, 2010) ........................ 71

Figure 33. Predicted $C_{1}$ Increase due to Pressurization of Internal Circulation Control Plenum . 72

Figure 34. Fluent Airfoil Model and Mesh Spacing ...................................................... 73

Figure 35. Velocity Contour Plot at $15^{\circ}$ AoA (Unmodified Airfoil) ........................................ 73

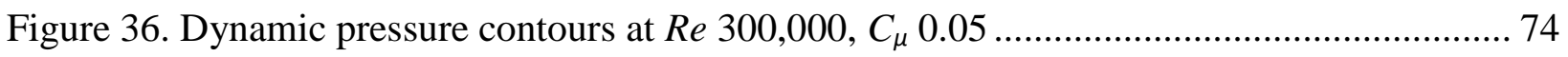

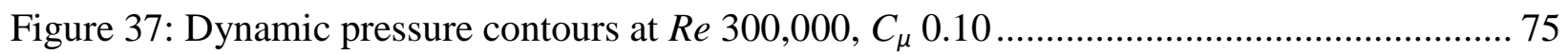

Figure 38. Computational Lift Coefficient Augmentation Prediction .................................... 76 
Figure 39. Propeller Design Technique 78

Figure 40. Region of Most Effective Circulation Control Geomerty (Kweder, Panther, Smith, 2010) 80

Figure 41. Clark-Y Dimensionless Cross-Sectional Area 82

Figure 42. Rounded Trailing Edge Modification for Clark-Y Airfoil ................................... 83

Figure 43. Radial Locations of Section Sweeps of Pressurization Plenum .............................. 84

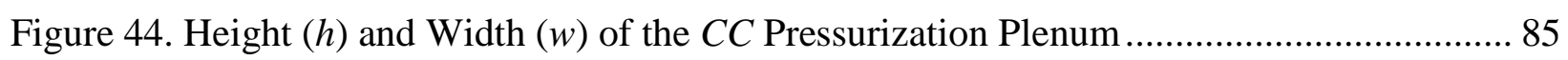

Figure 45. Exit Slot and Plenum of Circulation Controlled Propeller Blade............................. 86

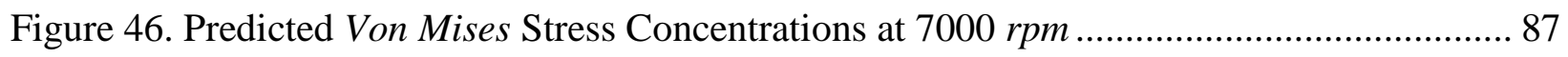

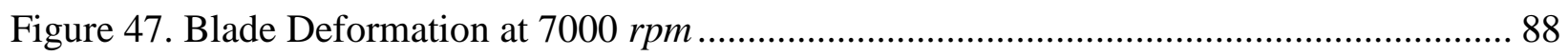

Figure 48. Increased Detail of the Predicted Circulation Control Exit Jet Deformation ............. 89

Figure 49. ProEngineer Representation of Circulation Control Augmented Propeller ............... 90

Figure 50. Fundamental Design of Capture Apparatus (not to scale).................................... 92

Figure 51. Experimental Flow Chart of Measurable Data and Testing outputs ....................... 94

Figure 52. Free-Stream Velocity Corrections Based on Tunnel Interference (Glauert, 1943) ..... 97

Figure 53. Wind Tunnel Assembly and Centripetal Fan, Pro-ENGINEER Model .................... 98

Figure 54. Motor and Propeller Experimental Test Stand Model......................................... 99

Figure 55. Relationship of Input Voltage to Shunt Voltage Reading .................................... 100

Figure 56. Test Stand and Motor Mounted in Wind Tunnel............................................ 101

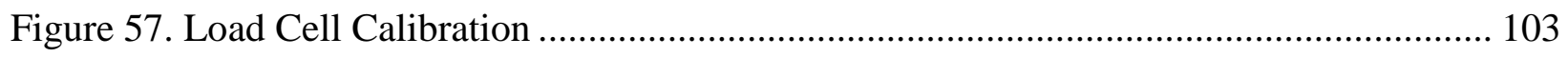

Figure 58 Unloaded Electric Motor Calibration ........................................................... 104

Figure 59. ESC Mounted on Experimental Test Stand .................................................... 106

Figure 60. Normal Flow inside the entrance of a Tunnel (White, 2006) ............................... 107 
Figure 61. Bubble Simulation of Velocity Cross-Section Inside a Tunnel (White, 2006) ......... 108

Figure 62. Velocity Measurements Taken at Centerline ( 0.737 meters from Bottom) ............ 109

Figure 63. Velocity Measurements Taken Above Centerline ( 1.143 meters from Bottom)...... 110

Figure 64. Velocity Measurements Taken Below Centerline ( 0.381 meters from Bottom)...... 110

Figure 65. Test Apparatus for Mounting Propeller........................................................... 111

Figure 66. Change in Angle-of-Attack in WPAFB Wind Tunnel........................................ 112

Figure 67. Force Balance Engineering Drawings ........................................................ 113

Figure 68. Propeller, Motor, and Force Balance Mounted in WPAFB Wind Tunnel................ 114

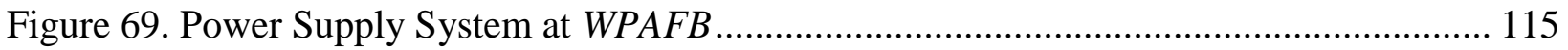

Figure 70. Characteristic Velocity of the Tunnel at WPAFB ............................................ 117

Figure 71. Close View of Airfoil Mesh Spacing for CFD analysis ..................................... 126

Figure 72. Complete CFD Analysis Grid Spacing ....................................................... 126

Figure 73. Initial Pressure Distribution of $C C$ Airfoil .................................................. 127

Figure 74. Velocity Magnitude of Airfoil Geometry at $R e=63649$ and $A o A=20^{\circ} \ldots \ldots \ldots \ldots \ldots . . . . .129$

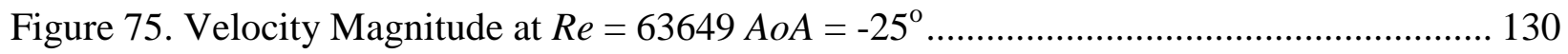

Figure 76. Circulation Control Augmented Lift Coefficient for Clark-Y Cross-Section ........... 131

Figure 77. Circulation Control Augmented Drag Coefficient for Clark-Y Airfoil Geometry.... 132

Figure 78. Theoretical Static Loading Compared to Experimental Results ............................ 137

Figure 79. Unaugmented Propeller Efficiency Performance Data ....................................... 139

Figure 80. Augmented Propeller Efficiency Performance Data .......................................... 139

Figure 81. Output Thrust Coefficient Comparison Between Augmented and Un-augmented

Propeller Experiments - WVU and WPAFB Reduced Results ................................... 140 
Figure 82. Input Torque Coefficient Comparison Between Augmented and Un-augmented Propeller Experimental Data - WVU and WPAFB Reduced Results

Figure 83. Power Coefficient Variation across Advance Ratio - WVU and WPAFB Reduced

Results 143

Figure 84. Full Spectrum Propeller Efficiency with Respect to Advance Ratio 144

Figure 85. Effect of Propeller Efficiency as Angle-of-Attack Changes 146

Figure 86. Full-Scale Ramp-Down Thrust Comparison with Respect to Time..... 148

Figure 87. Full-Scale Ramp-Down Torque Comparison with Respect to Time. 148

Figure 88. Full-Scale Ramp-Up Thrust Comparison with Respect to Time 149

Figure 89. Full-Scale Ramp-Up Torque Comparison with Respect to Time 150

Figure 90. Gathered Lift Coefficient Data on Unaugmented Clark-Y Two-Dimensional Airfoil 152

Figure 91. Unaugmented Two-Dimensional Drag Coefficient Data for Clark-Y Airfoil 153

Figure 92. Thrust Force Analysis of Unaugmented and Augmented Propellers 154

Figure 93. Input Torque Required for Unaugmented and Augmented Propellers..... 155

Figure 94. Overall Efficiency of Propellers Analyzed through Blade Element Model 156

Figure 95. Experimental vs Computational Propeller Efficiency Results 157

Figure 96. Manufacturer Performance Factors of the Rimfire Electric Motor (http://www.peakeff.com) 158

Figure 97. Unaugmented Propeller Efficiency Data Collection Error Analysis 160

Figure 98. Augmented Propeller Efficiency Data Error Analysis 161

Figure 99. Statistical Analysis of Gathered Experimntal Data 162

Figure 100. Cincinnati Fan Dimensions 181 
Figure 101. Specifications of Materials used in the FORTUS 900mc Rapid Prototyping Machine 183

Figure 102. Specifications of the Konica Minolta Range 7 Laser Scanner ............................ 185

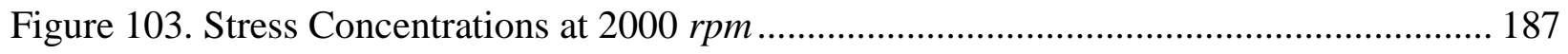

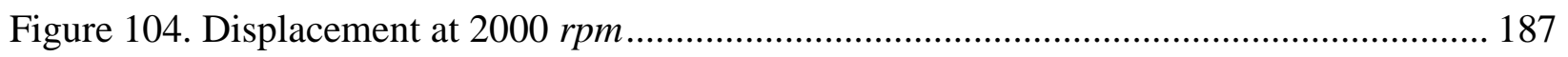

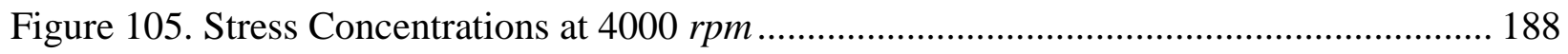

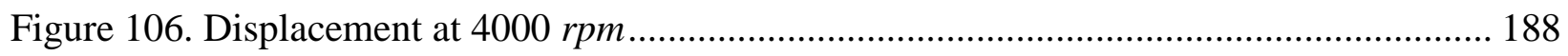

Figure 107. Stress Concentrations at 9000 rpm (Failure Predicted) ...................................... 189

Figure 108. Displacements at 9000 rpm (Failure Predicted) ............................................. 189

Figure 109. Brushless Motor Specifications ................................................................. 190

Figure 110. Hobbywing Platinum Pro ESC Wiring Diagram ............................................. 191

Figure 111. Governor Mode Throttle Volume.................................................................. 192

Figure 112. Computer to Program Box to ESC Wiring Diagram ......................................... 193

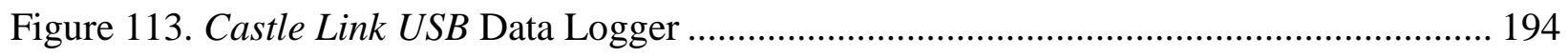

Figure 114. Castle Creations Phoneix ICE-50 Electronic Speed Controller............................ 195

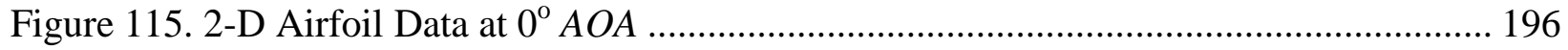

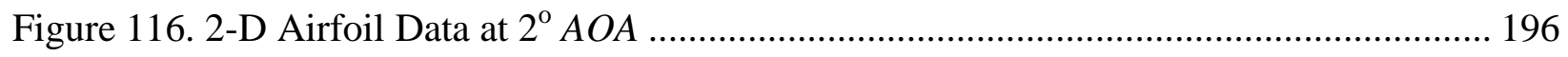

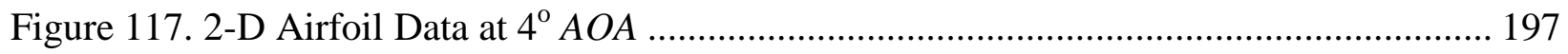

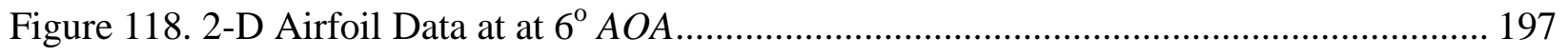

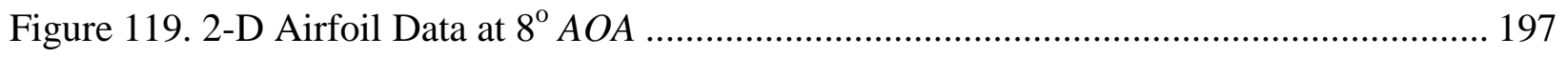

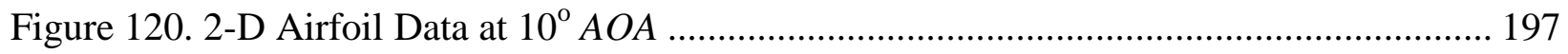

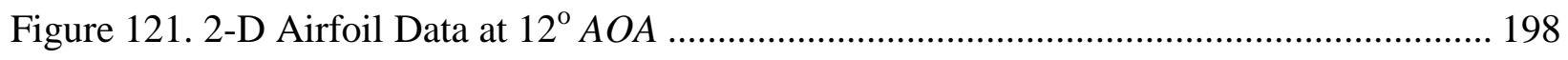

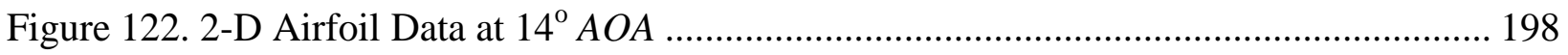




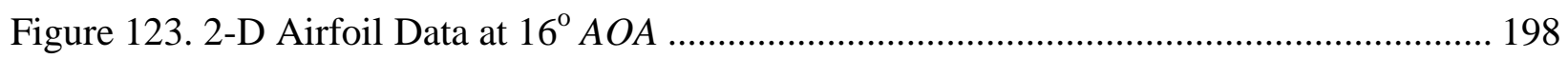

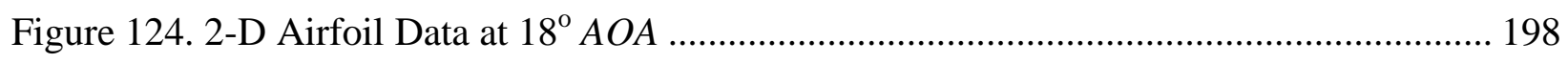

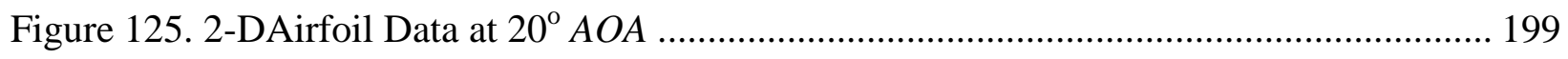

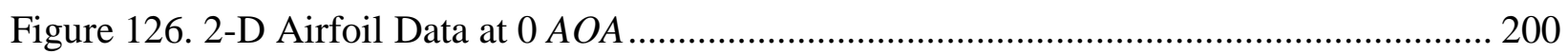

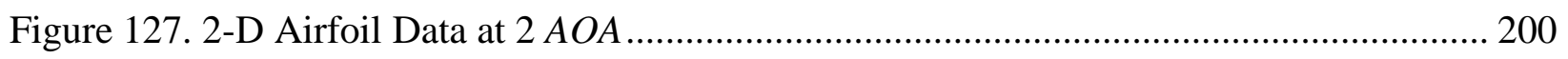

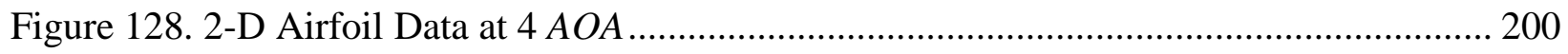

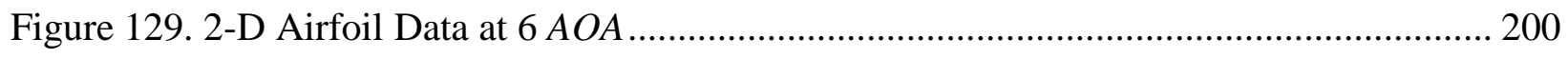

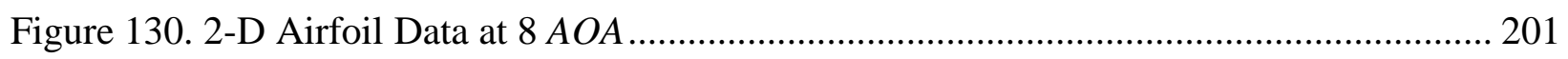

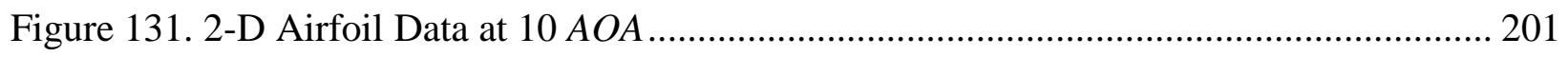

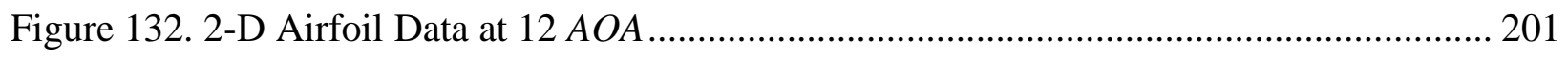

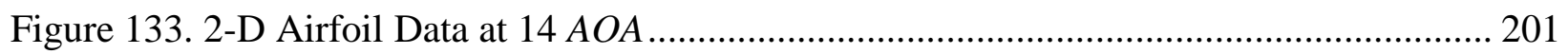

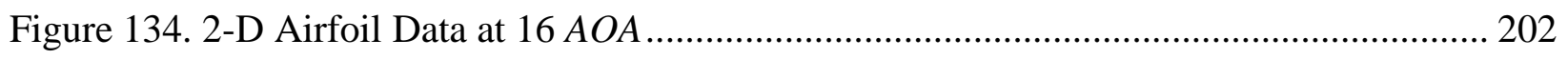

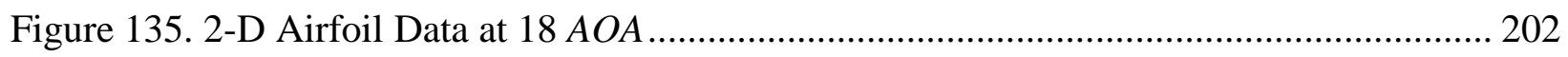

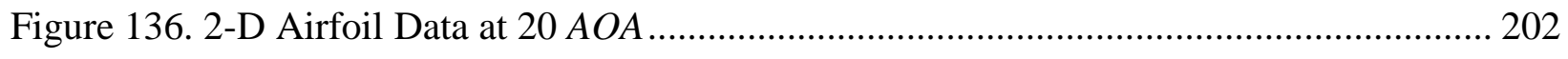

Figure 137. $C_{l}$ vs Angle-of-Attack for Clark-Y Cross-Section ……………………................. 203

Figure 138. $C_{d}$ vs Angle-of-Attack for Clark-Y Cross-Section.................................................. 204

Figure 139. Output Thrust versus Advance Ratio at Tunnel Speed (Unaugmented Prop) ......... 205

Figure 140. Output Thrust Versus Advance Ratio at Tunnel Speed (Augmented Prop)............ 206

Figure 141. Output Thrust versus Advance Ratio at Tunnel Speed (Unaugmented Prop) ......... 207

Figure 142. Output Thrust versus Advance Ratio at Tunnel Speed (Augmented Prop)............. 207

Figure 143. Input Torque Versus Advance Ratio at Tunnel Speed (Unaugmented Prop) .......... 209

Figure 144. Input Torque Versus Advance Ratio at Tunnel Speed (Augmented Prop) ............. 209 
Figure 145. Input Torque with Respect to Advance Ratio Over Tunnel Speed (Unaugmented Prop) 210

Figure 146. Input Torque with Respect to Advance Ratio Over Tunnel Speed (Augmented Prop)

Figure 147. Thrust Coefficient versus Advance Ratio....................................................... 212

Figure 148. Coffecient of Torque versus Advance Ratio of Experimental Propellers ............. 213

Figure 149. Propeller Power Coefficient versus Advance Ratio ............................................ 213

Figure 150. ESC Data at Tunnel Velocity of 10 Miles per Hour ......................................... 215

Figure 151. ESC Data at Tunnel Velocity of 20 Miles per Hour ......................................... 215

Figure 152. ESC Data at Tunnel Velocity of 30 Miles per Hour ........................................ 216

Figure 153. ESC Data at Tunnel Velocity of 10 Miles per Hour .......................................... 217

Figure 154. ESC Data at Tunnel Velocity of 20 Miles per Hour ......................................... 218

Figure 155. ESC Data at Tunnel Velocity of 30 Miles per Hour ....................................... 218

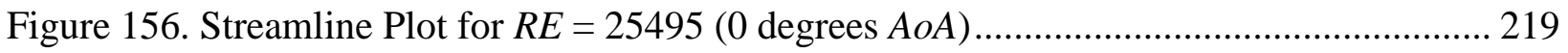

Figure 157. Pressure Contour Plot for $R E=25495(0$ degrees $A o A)$.................................. 220

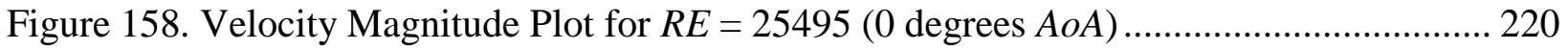

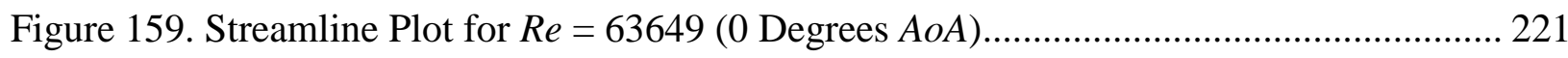

Figure 160. Pressure Contour Plot for $R e=63649$ (0 Degrees $A o A)$................................... 221

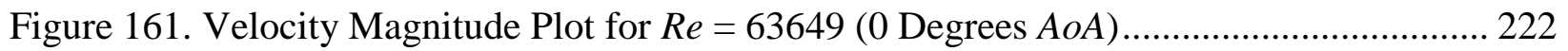

Figure 162. Stream Line Plot at $R E=127298(0$ Degrees $A o A)$......................................... 222

Figure 163. Pressure Contour Plot at $R E=127298$ (0 Degrees $A o A)$.................................. 223

Figure 164. Velocity Magnitude Plot at $R E=127298$ (0 Degrees $A o A)$............................... 223 


\section{List of Tables}

Table 1. Blade Plenum Reference Geometries ................................................................ 85

Table 2. Testing Schedule and Experimental Data Runs.................................................. 119

Table 3. Advance Ratio Testing Schedule at WPAFB/AFRL Wind Tunnel ............................ 121

Table 4. Angle-of-Attack Test Schedule at WPAFB/AFRL Vertical Tunnel........................... 122

Table 5. Baseline Propeller Thrust Capabilities- ( $D=24$ Inch, $\boldsymbol{P} *=10)$ (Zinger, Inc., 2010) .... 124 


\section{Nomenclature}

Symbol

A

$A B S$

AC

AFRL

AHS

AIAA

AoA

ASEE

ASME

BET

c

C $\underline{\text { Description }}$

Disk Area

Acrylonitrile Butadine Styrene

Alternating Current

Air Force Research Laboratory

American Helicopter Society

American Institute of Aeronautics and Astronautics

Angle-of-Attack

American Society of Engineering

Education

American Society of Mechanical

Engineers

Blade Element Theory

Chord

Tunnel Area

$\underline{\text { Units (Imperial) }}$

$\underline{\text { Units (Metric) }}$

Foot $^{2}$

Degree/Radian Degree/Radian

Foot

Meter

Foot $^{2}$

Meter $^{2}$ 


\begin{tabular}{|c|c|}
\hline$C C$ & Circulation Control \\
\hline $\mathrm{CCHB}$ & Circulation Control Helicopter Blade \\
\hline$C_{d}$ & Two-Dimensional Drag Coefficient \\
\hline$C_{D}$ & Three-Dimensional Drag Coefficient \\
\hline$C F D$ & Computational Fluid Dynamics \\
\hline$C_{l}$ & Two-Dimensional Lift Coefficient \\
\hline$C_{L}$ & Three-Dimensional Lift Coefficient \\
\hline$C_{m}$ & Moment Coefficient \\
\hline$C_{p}$ & Pressure Coefficient \\
\hline$C_{P *}$ & Power Coefficient \\
\hline$C_{Q}$ & Torque Coefficient \\
\hline$C_{T}$ & Thrust Coefficient \\
\hline$C_{\mu}$ & Blowing Coefficient \\
\hline$D$ & Diameter \\
\hline$D C$ & Direct Current \\
\hline$E S C$ & Electronic Speed Controller \\
\hline
\end{tabular}


FORT Facility for Outdoor Rotor Testing

$g$

$h / c$

$I$

$J$

$k V$

$L i-P o$

$L / r$

$M$

$n$

$N A C A$

NAL National Aeronautical Laboratory

NASA

$\mathrm{Ni}-\mathrm{MH}$

Gravitational Constant

Slot Height to Chord Ratio

Current

Advance Ratio

Rotations per Input Volt

Lithium Polymer

Tunnel Location

Mach Number

Rotational Speed

National Advisory Committee for Aeronautics

National Aeronautics and Space

Administration

Nickel-Metal Hydride
Foot/Second ${ }^{2} \quad$ Meter/Second ${ }^{2}$

Ampere

Ampere

RPM/Volt

RPM/Volt

Rotations/Second Rotations/Second 


\begin{tabular}{|c|c|c|c|}
\hline NSRDC & $\begin{array}{l}\text { Naval Ship Research and } \\
\text { Development Center }\end{array}$ & - & - \\
\hline NSWC & Naval Surface Warfare Center & - & - \\
\hline$P$ & Pressure & Pound/Foot ${ }^{2}$ & Newton/Meter ${ }^{2}$ \\
\hline$P_{*}$ & Propeller Pitch & Inch/Revolution & Meter/Revolution \\
\hline$P C$ & Poly-Carbonate & - & - \\
\hline PIV & Particle Image Velocimetry & - & - \\
\hline $\operatorname{Pr}$ & Prandtl Number & - & - \\
\hline$Q$ & Torque & Foot-Pounds & Newton-Meter \\
\hline$R$ & Total Radius & Foot & Meter \\
\hline$R_{*}$ & Resistance & Ohm & Ohm \\
\hline$R_{x}$ & Ideal Gas Constant & $\begin{array}{l}\text { Foot-Pound/Slug- } \\
\text { Rankine }\end{array}$ & $\begin{array}{l}\text { Joule/Kilogram- } \\
\text { Kelvin }\end{array}$ \\
\hline$r$ & Radial Element & Foot & Meter \\
\hline$r / c$ & $\begin{array}{c}\text { Trailing Edge Radius to Airfoil } \\
\text { Chord Ratio }\end{array}$ & - & - \\
\hline
\end{tabular}




\begin{tabular}{|c|c|c|c|}
\hline$r / R$ & Blade Radial Location & - & - \\
\hline$S A E$ & Society of Automotive Engineers & - & - \\
\hline Temp & Temperature & Rankine & Kelvin \\
\hline$T$ & Thrust & Pound & Kilogram \\
\hline$t$ & Time & Second & Second \\
\hline$t$ & Thickness & Foot & Meter \\
\hline$t / c$ & $\begin{array}{l}\text { Airfoil Maximum Thickness-to- } \\
\text { Chord-Ratio }\end{array}$ & - & - \\
\hline$u$ & X-Velocity Component & Foot/Second & Meter/Second \\
\hline$V$ & Velocity & Foot/Second & Meter/Second \\
\hline$v$ & Y-Velocity Component & Foot/Second & Meter/Second \\
\hline$V^{*}$ & Voltage & Volt & Volt \\
\hline V/STOL & Vertical/Short Take-Off and Landing & - & - \\
\hline$w$ & Z-Velocity Component & Foot/Second & Meter/Second \\
\hline$W P A F B$ & Wright-Patterson Air Force Base & - & - \\
\hline$y$ & Distance Above the Surface & Foot & Meter \\
\hline
\end{tabular}




\section{Greek Symbols}

Symbol

$\alpha$

$\beta$

$\gamma$

$\frac{\partial}{\partial t}$

$\frac{\partial}{\partial x}$

$\frac{\partial}{\partial y}$

$\frac{\partial}{\partial z}$

$\vartheta$

$\mu$

$\eta$

$v$

$\rho$ $\underline{\text { Description }}$

Angle-of-Attack

Pitch Angle

Specific Heat Ratio

Partial Derivative with Respect to Time

Partial Derivative X-Direction

Partial Derivative Y-Direction

Partial Derivative Z-Direction

Induced Angle

Dynamic Viscosity

Efficiency

Kinematic Viscosity

Density $\underline{\text { Units (Imperial) }}$ Units (Metric)

Degree/Radian Degree/Radian

Degree/Radian Degree/Radian

$-$

$-$

$-$

Degrees

Degrees

Pound- Kilogram-

Second/Foot ${ }^{2} \quad$ Second/Meter ${ }^{2}$

Foot ${ }^{2} /$ Second $\quad$ Meter $^{2} /$ Second

Pound/Foot ${ }^{3} \quad$ Kilogram/Meter ${ }^{3}$ 


\begin{tabular}{|c|c|c|c|}
\hline$\tau_{x x}$ & Normal Shear Stress X-Direction & Pound/Foot ${ }^{2}$ & Kilogram/Meter ${ }^{2}$ \\
\hline$\tau_{y y}$ & Normal Shear Stress Y-Direction & Pound/Foot ${ }^{2}$ & Kilogram/Meter ${ }^{2}$ \\
\hline$\tau_{x y}$ & XY Shear Stress & Pound/Foot ${ }^{2}$ & Kilogram/Meter ${ }^{2}$ \\
\hline$\psi$ & Effective Pitch Angle & Degrees & Degrees \\
\hline$\Omega$ & Rotational Velocity & Rotations/Minute & Rotations/Minute \\
\hline
\end{tabular}




\section{Subscripts and Superscripts}

$\underline{\text { Symbol }}$

A

$b$

$c$

$D$

$d$

$j$

$L$

$P_{\infty}$

$r$

$T$

$v_{\infty}$

$x$

$y$

$z$ $\underline{\text { Description }}$

$\underline{\text { Units }}$

With Respect to Wing Area

Span

Chord

With Respect to Drag

Plenum Exit

Jet

With Respect to Lift

With Respect to Ambient Pressure

Required

With Respect to Temperature

With respect to Free-stream Velocity

X-direction

Y-direction

Z-direction 
Free-stream 


\section{Introduction}

Circulation control $(C C)$ is a lift augmentation methodology that can be used in a variety of fluid dynamic systems, such as, on the wing of an aircraft. Applications of this technology have been in the research and development phase since the 1960's with the primary emphasis on fixed-wing aircraft where the initial implementations of this technology were referred to as "blown flaps." Circulation control increases the near surface velocity of airflow over a rounded surface of an object, typically a slightly modified airfoil. This is primarily achieved though the addition of a jet of air to a specially designed aircraft wing using a series of blowing slots that eject pressurized high velocity (faster than the free-stream velocity) jets of air over the trailing edge and/or leading edge.

The most prevalent design for a circulation controlled wing, has a rounded trailing edge, and ejects the air tangential to the surface, inducing the Coandă Effect. The Coandă Effect is typically characterized by a boundary layer jet that remains attached to the wing surface longer than a conventional wing and thus increases the virtual length, camber, and the angle-of-attack of the airfoil by entraining the free-stream air near the jet. The jet blowing over the rounded trailing edge increases the circulation of the free-stream around the airfoil, or similar object, increasing the aerodynamic forces experienced. As the circulation control airflow entrains free-stream air, the interaction between the object and the surrounding fluid alters the pressure distribution along the surface of the airfoil, another indication of the enhanced forces experienced by the object.

The principal motivation for the use of circulation control has been to increase the lifting force when large lifting forces and/or slow speeds are beneficial, such as at take-off and landing. On current fixed-wing aircraft, wing flaps and leading edge slats are used during landing and on 
take-off to increase lift generation. The benefit of the circulation control wing is that no additional skin friction drag is created by the increased surface area produced by the movement of conventional surfaces into the airflow around the wing. This results in a greatly increased lift coefficient. However, as with any lifting surface, the use of circulation control increases the induced drag of the airfoil in proportion to the square of the lift coefficient (Bertin, 2002). Additionally, these active circulation control systems require energy to pump the fluid, along with the additional hardware, weight and system complexity which can reduce the effectiveness achieved by the additional lift.

Circulation control has also been adapted for use on rotary-wing aircraft. In the early years of circulation control there were two critical design issues with the addition of circulation control to a rotating body such as a propeller or helicopter rotor. The first issue was the exposure of the rotors on a helicopter to a moderate range of angles-of-attack, $0-50^{\circ}$ (Stepniewski, 1981) caused by the inflow of air through the rotor plane. Through the study of high angles-of-attack $\left(20-35^{\circ}\right)$ in wind tunnel testing, it is now possible to predict the behavior of the rotor blade through its rotation (Stepniewski, 1981).

The second obstacle in the prior applications of circulation control to a helicopter main rotor was the inability to achieve the response times necessary to effectively use circulation control on a rotary-wing aircraft to accommodate the asymmetry of lift experienced during maneuvering flight. With the recent development (in the last 10 years) of smart materials, a specifically designed near surface piezoelectric valve (Smith, et al, 2002) was conceived, enabling the concept of using circulation control on the blades of a rotary-wing aircraft. Through the use of these and similar types of smart materials, the response time of circulation control can 
be reduced, opening circulation control to a wider array of applications including rotorcraft and propellers.

A further consideration of circulation control applications to rotorcraft is managing the power needed to supply the augmented airflow. An active circulation control system uses an internal pumping system which can draw power from the original aircraft power plant or from another additional power source, such as a compressor, to pressurize the air plenums in order to implement circulation control on the aerodynamic body. In the past, for some of these applications, the additional weight and cost of components far surpassed the benefits derived from the use of circulation control. While new materials and control strategies have made available a great variety of applications, using active circulation control for some simpler systems can still be problematic.

According to a report published by National Aeronautics and Space Administration (NASA), in conjunction with The Bionetics Corporation in June 1981, early research was completed examining the effect that circulation control had on propeller applications (Braslow, 1981). This report specifically found that the overall system provided "greater than 7 percent reduction in fuel usage at cruise." This result was found on a system applied to a "puller-type" 1.83 meter (6 foot) diameter propeller, with an added external pumping system (actively pressurized circulation control plenum). This added pumping system siphons power from the engine and adds weight to the overall aircraft, rendering the trade-offs of adding the system to the aircraft unreasonable. However, without the weight and complexity of another pressurization system it may be more than adequate to warrant a passively pressurized system, the object of this study, to be used in future research applications. 
With the development and continuous improvement of numerous unmanned aerial vehicles $(U A V s)$, wing and propeller performance enhancement is desirable. Though potentially beneficial, the application of the active circulation control to either a $U A V$ 's wing or propeller, may create technical difficulties by interfering with the supply of air to the circulation control blowing slot. Thus, a passive system in which air can be supplied to a strategically placed circulation control blowing slot could be used to enhance the performance of these aircraft. Although the use of circulation control slots on the wing will require the addition of an auxiliary pumping system, it is envisioned that the use of circulation control on the main propeller would not require this extra system and might create a self-contained air supply of its own.

This passive system can take advantage of the pressure differential upstream and downstream of the propeller plane, forward air velocity, and centripetal acceleration to pressurize the internal plenum of the circulation control system. In doing so, no additional power source will be necessary to augment the propeller of the aircraft. 


\section{Research Objectives}

The goal of this study was to implement a passive circulation control system inlet to an already existing propeller design. As such, it was necessary to optimize the propeller system dimensions based on available materials for construction as well as industry standards for propeller geometry. It was necessary to study the pertinent dimensions and sizing criteria of the propeller, listed below, on an application basis, and take into account the individual effect each had on the entire system. This method was employed in order to identify the most influential of the geometry constraints and ultimately to build a working experimental model.

- Exit Slot Placement Along the Radius

- Spectrum of Rotational Speeds

- Exit Slot Geometry

- Internal Plenum Dimensions

It was also the objective of this research to prove that the application of a passive circulation control system can be beneficial in increasing the overall thrust of an aircraft over a full spectrum of advance ratios experienced by the propeller system. The hypothesis being that the capture and rerouting of stagnation air from the front plane of the propeller while in use would decrease the pressure drag on the rotating body, and then be used to enhance the circulation around the propeller blade at the trailing edge exit slot. This study focused on the availability of using this type of system in instances where an external mechanical/electrically driven pumping device was not available. In order to accurately prove this premise, both an experimental model, and an analytical model needed to be constructed and analyzed. 


\section{Literature Review}

This section summarizes several basic aerodynamic concepts and flow physics for use in wind tunnel testing, as well as prior research into circulation control wings, and stall angles. This summary starts with basic fluid flow understanding, boundary layer theory, and summarizes some of the applicable sciences and theories used when dealing with circulation control, such as Coandă surfaces and the Kutta Condition. Prior research on circulation control is then discussed in detail summarizing the beginnings of research into this science and continuing into more recent applications. Some basic propeller and airscrew sciences are discussed in the final section of this review, including design basics as well as insight into previous research and development.

\section{Fluid Flow Basics}

Wind tunnel investigations typically begin with an understanding of the fundamental fluid flow relationships, namely, the continuity and conservation of momentum equations. The two-dimensional, flow form of the shear stress equations, as shown in Equation 1, Equation 2, and Equation 3 are functions of density $(\rho)$, viscosity $(\mu)$, time $(t)$, the surface radius of curvature $(r)$, height above the surface $(y)$, the $\mathrm{x}$-directional fluid velocity $(u)$, and the $\mathrm{y}$-component of velocity $(v)$ (Ambrosiani, 1971). These equations define the normal $\left(\tau_{x x}, \tau_{y y}\right)$ and shear stresses $\left(\tau_{x y}\right)$ to be used in the conservation of momentum equations.

$$
\begin{array}{cc}
\tau_{x x}=2 \mu\left[\frac{r}{r+y} \frac{\partial u}{\partial x}+\frac{u}{R+y}-\frac{1}{3}\left(\frac{r}{r+y} \frac{\partial u}{\partial x}+\frac{\partial v}{\partial y}+\frac{v}{r+y}\right)\right] & \text { Equation 1 } \\
\tau_{y y}=2 \mu\left[\frac{\partial v}{\partial y}-\frac{1}{3}\left(\frac{r}{r+y} \frac{\partial u}{\partial x}+\frac{\partial v}{\partial y}+\frac{v}{r+y}\right)\right] & \text { Equation 2 } \\
\tau_{x y}=\mu\left[\frac{r}{r+y} \frac{\partial v}{\partial y}+\frac{r+y}{r} \frac{\partial\left(\frac{r}{r+y} u\right)}{\partial y}\right] & \text { Equation 3 }
\end{array}
$$


The continuity, Equation 4, and conservation of momentum equations, Equation 5 and Equation 6, provide the basic steps for theoretical calculation of the directional velocities predicted for a moving fluid. The x-direction conservation of momentum, Equation 5, uses the results of Equation 1 and Equation 3. Conversely, the y-direction conservation, Equation 6, uses the results from Equation 1 and Equation 2, illustrated above.

$$
\begin{array}{cc}
\frac{\partial \rho}{\partial t}+\frac{r}{r+y} \frac{\partial(\rho u)}{\partial x}+\frac{r}{r+y} \frac{\partial\left(\frac{r+y}{r}(\rho v)\right)}{\partial y}=0 & \text { Equation 4 } \\
\rho\left[\frac{\partial u}{\partial t}+\frac{r}{r+y} u \frac{\partial u}{\partial x}+v \frac{\partial u}{\partial y}+\frac{u v}{r+y}\right]+\frac{r}{r+y} \frac{\partial P}{\partial x}-\frac{r}{r+y} \frac{\partial \tau_{x x}}{\partial x}-\frac{\partial \tau_{x y}}{\partial y}-\frac{2}{r+y} \tau_{x y}=0 & \text { Equation } 5 \\
\rho\left[\frac{\partial v}{\partial t}+\frac{r}{r+y} u \frac{\partial v}{\partial x}+v \frac{\partial v}{\partial y}+\frac{u^{2}}{r+y}\right]+\frac{\partial P}{\partial x}-\frac{r}{r+y} \frac{\partial \tau_{x x}}{\partial x}-\frac{\partial \tau_{y y}}{\partial y}-\frac{1}{r+y}\left[\tau_{y y}-\tau_{x x}\right]=0 & \text { Equation } 6
\end{array}
$$

These equations predict how particular particles or objects behave in a closed system. The momentum of any non-deformable object is conserved and therefore the center of mass will move with a uniform velocity unless acted upon by an outside force. This theory includes the movement of air molecules in a wind tunnel environment, where the particles will behave in a consistent manner until influenced by an object outside of its original closed system in the wind tunnel, such as an aerodynamic body.

\section{Flow of a Jet}

A jet is a continuous stream of fluid that is injected into a surrounding fluid, usually via some nozzle or similar opening. Frequently, an aerodynamicist's goal is to increase the kinetic energy of the flowing medium at the expense of its pressure energy and/or internal energy. The jet can also be used to energize the fluid it is flowing into (i.e. circulation control). Figure 1 shows the interaction between the boundary layer around an object and a wall jet (Meyer, 1972). The figure illustrates how a jet can add energy to the boundary layer in the form of increased velocity which allows the flow to move around a curved surface instead of separating. 


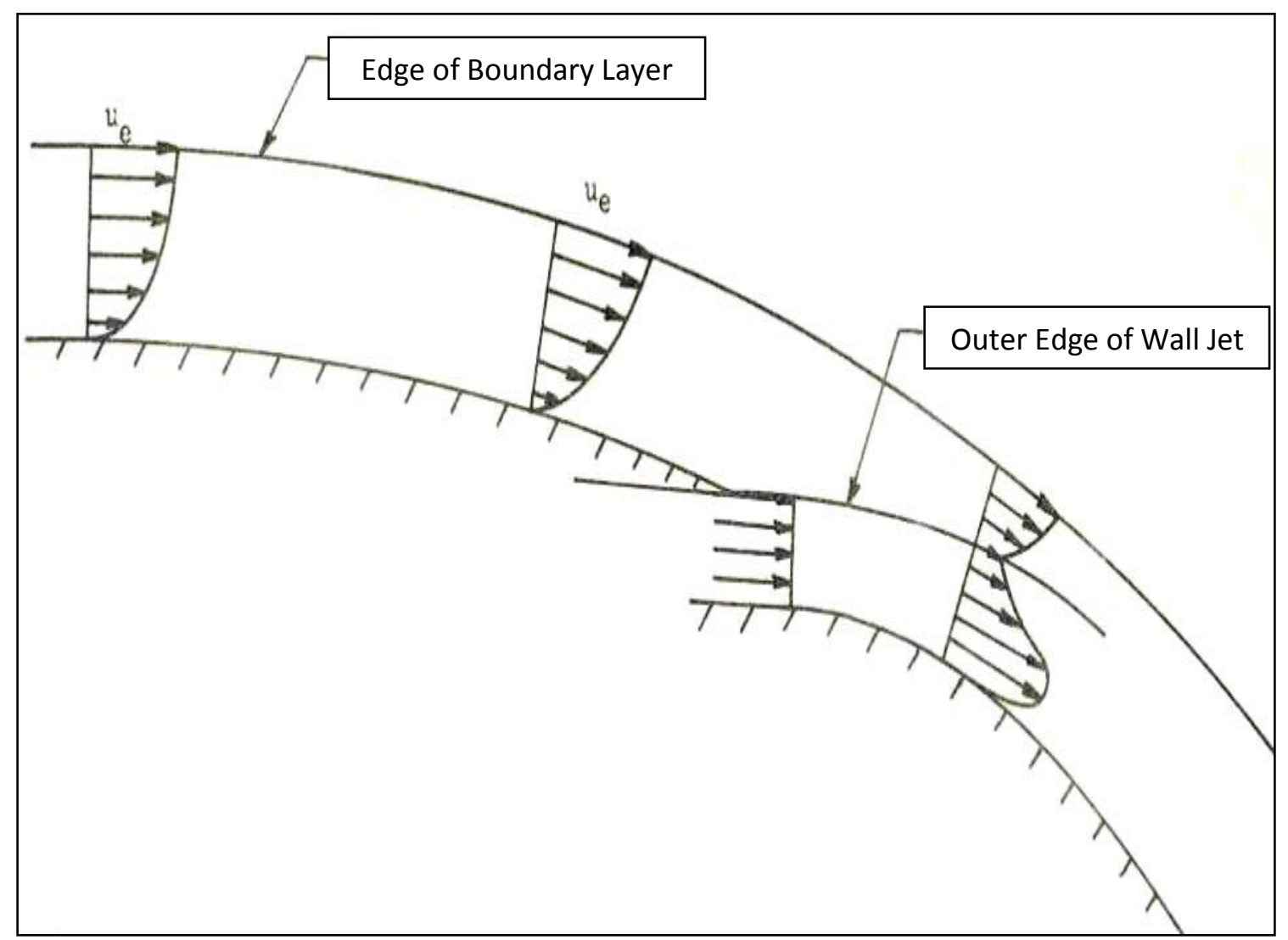

Figure 1. Effect of a Wall Jet on Boundary Layer (Meyer, 1972)

There are several different types of nozzles used in aerodynamics. Most can be described as convergent (narrowing down from a wide diameter to a smaller diameter in the direction of the flow) or divergent (expanding from a smaller diameter to a larger one). Convergent nozzles accelerate subsonic fluids, until the nozzle pressure ratio is such that the flow reaches sonic velocity at the choke, or narrowest point of the flow. Increases in the nozzle pressure ratio above this point no longer increases the throat Mach Number (limited to a value of unity). Downstream (i.e. external to the nozzle) the flow is free to expand to supersonic velocities. Conversely, divergent nozzles decrease the velocity of subsonic flows.

There is also a third type, de Laval Nozzle, which has a convergent section followed by a divergent section which is often called a 'convergent-divergent' nozzle. Convergent-divergent nozzles can accelerate fluids that have choked in the convergent section to supersonic speeds. 
This acceleration process is more efficient than allowing a convergent nozzle to expand supersonically externally, thus a 'convergent-divergent' nozzle is often used as a velocity control in wind tunnels. In addition to being used in supersonic applications, the 'convergent-divergent' nozzle is also efficient at less than sonic speeds.

\section{Basic Boundary Layer Theory}

First discovered in 1904 by Ludwig Prandtl, the boundary layer of an object immersed in a fluid is one of the two regions of flow (White, 2006). The boundary layer is the portion immediately touching the surface of the object and is the region in which the viscous force of the fluid is the most predominant. Because of the domination of the viscous force on the object, this is also where the greatest amount of drag force is experienced. There are two different thicknesses used to describe the boundary layer around an aerodynamic body: the velocity and the thermal boundary layers. The thickness of the velocity boundary layer is defined as the distance from the solid body at which the flow velocity is 99 percent of the free-stream velocity, that is, the velocity that is calculated at the surface of the body in an inviscid flow solution (White, 2006).

The no-slip condition requires the flow velocity at the surface of a solid object be equal to

the speed of the object and the fluid temperature be equal to the temperature of the object's surface. The flow velocity will then increase rapidly within the boundary layer, governed by the Navier-Stokes equations, given as, Equation 7, Equation 8, Equation 9, and Equation 10.

The thermal boundary layer thickness is similarly defined as the distance from the body at which the temperature is 99 percent of the temperature found from an inviscid solution (White, 2006). The ratio of the two thicknesses is governed by the Prandtl Number $(\operatorname{Pr})$. The simplified form of the three-dimensional Navier-Stokes equations, for estimating the forces produced by fluids, again, are shown below (Mills, 1999). 


$$
\begin{array}{cc}
\frac{\partial u}{\partial x}+\frac{\partial v}{\partial y}+\frac{\partial w}{\partial z}=0 & \text { Equation 7 } \\
\frac{\partial u}{\partial t}+u \frac{\partial u}{\partial x}+v \frac{\partial u}{\partial y}+w \frac{\partial u}{\partial z}=-\frac{1}{\rho} \frac{\partial p}{\partial x}+v\left(\frac{\partial^{2} u}{\partial x^{2}}+\frac{\partial^{2} u}{\partial y^{2}}+\frac{\partial^{2} w}{\partial z^{2}}\right)+\rho g_{x} & \text { Equation 8 } \\
\frac{\partial v}{\partial t}+u \frac{\partial v}{\partial x}+v \frac{\partial v}{\partial y}+w \frac{\partial v}{\partial z}=-\frac{1}{\rho} \frac{\partial p}{\partial y}+v\left(\frac{\partial^{2} v}{\partial x^{2}}+\frac{\partial^{2} v}{\partial y^{2}}+\frac{\partial^{2} w}{\partial z^{2}}\right)+\rho g_{y} & \text { Equation 9 } \\
\frac{\partial w}{\partial t}+u \frac{\partial w}{\partial x}+v \frac{\partial w}{\partial y}+w \frac{\partial w}{\partial z}=-\frac{1}{\rho} \frac{\partial p}{\partial z}+v\left(\frac{\partial^{2} w}{\partial x^{2}}+\frac{\partial^{2} w}{\partial y^{2}}+\frac{\partial^{2} w}{\partial z^{2}}\right)+\rho g_{z} & \text { Equation 10 }
\end{array}
$$

These equations are used to model a large number of gas and liquid flow phenomena in the world including weather patterns, ocean currents, and flow over an aerodynamic body. These equations have been the backbone of aerodynamic research since their introduction by Claude Louis Navier and George Gabriel Stokes in 1822 (White, 2006). Coupled with Newton's conservation of momentum, when explicitly solved, they relate variables such as velocity, pressure, and viscosity, to one another.

\section{Coandă Effect}

A complex type of flow, where there is a deflection of a fluid jet by a curved surface near the boundary, is known as the Coandă Effect. When fluids act under this principle, they move in streamlines over a convex surface away from the original path. This resulting deflection is created by the balance of the inertial force in the jet and the reduced pressure at the wall, caused by the jet velocity (Englar, 1975). One application which can be replicated in everyday life, demonstrating the Coandă Effect, is by turning on a faucet and moving the convex side of a tablespoon toward the jet of water. As the spoon gets close enough to the fluid's boundary, the streamlines will change, thus, deflecting the water over the spoon's surface.

The Coandă Effect can also be explained as a wall jet which energizes the boundary layer creating a lower pressure near the surface than in the free-stream. This pressure sink, delays the separation of the free-stream from the aerodynamic surface. As the wall jet thickens due to the additional entrained mass, from free-stream and viscous dissipation, the low pressure at the wall 
increases until the surface and free-stream pressures equalize, at which point jet separation then occurs (Ambrosiani, 1971). The early stages of circulation control research were based on the premise that the specially designed wings with ejectors would help adhere the flow to the airfoil surface through the Coandă Effect and increase the distance along the wing chord at which the separation point occurred.

A physical representation of the Coand $\breve{a}$ Effect is shown by the two-dimensional flow over a circular cylinder in Figure 2 (Newman, 1961). In this situation, the jet is shown on the bottom of the cylinder as having a velocity $(U)$. The jet makes the flow bend upwards and consequently it flows further around the cylinder, delaying the separation of the boundary layer.

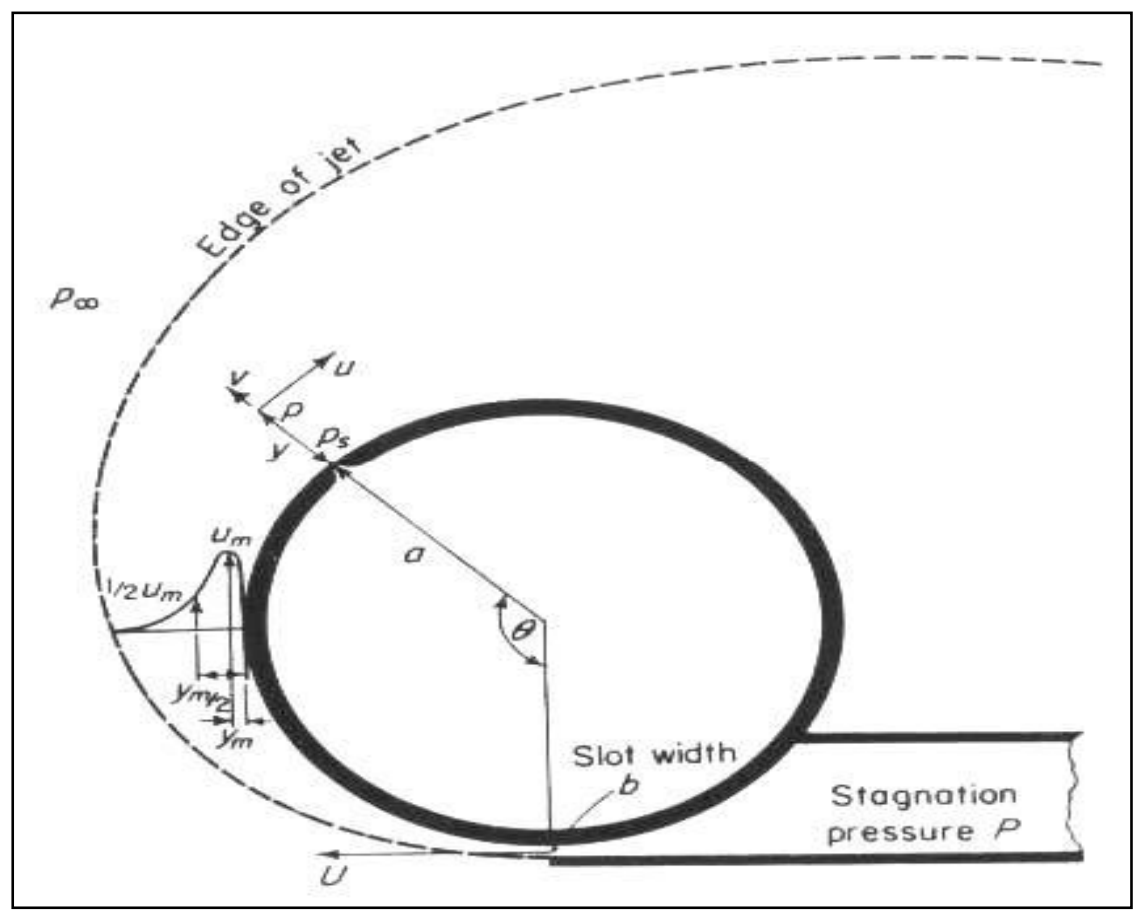

Figure 2. Flow of a Two-Dimensional Jet Around a Circular Cylinder (Newman, 1961)

\section{Kutta Condition}

M. W. Kutta was a German mathematician who developed the widely accepted theory defining the location of the rear stagnation point of an airfoil as being dependent upon the circulation around the airfoil. Kutta's condition states that if the flow leaves a sharp trailing edge 
of an airfoil smoothly, the velocity there is finite (Katz, 2001). Figure 3 shows the three different ways that the flow can theoretically leave the trailing edge of an airfoil. Following Kutta's hypothesis, it can be seen how the rear stagnation point shifts to a different place on the airfoil depending on the amount of circulation over the wing. Figure 3 (a) shows the resultant flow when there is no circulation, Figure 3 (b) shows the perfect amount of circulation around an airfoil, and Figure 3 (c) shows more than enough circulation to satisfy the Kutta Condition. Because the airfoil tested for this work has a rounded trailing edge, it is expected that the flow over the trailing edge of the airfoil, while circulation control is being employed, will act similar to the situation seen in Figure 3 (c) (Katz, 2001), entraining airflow and taking advantage of the Coandă Effect. 


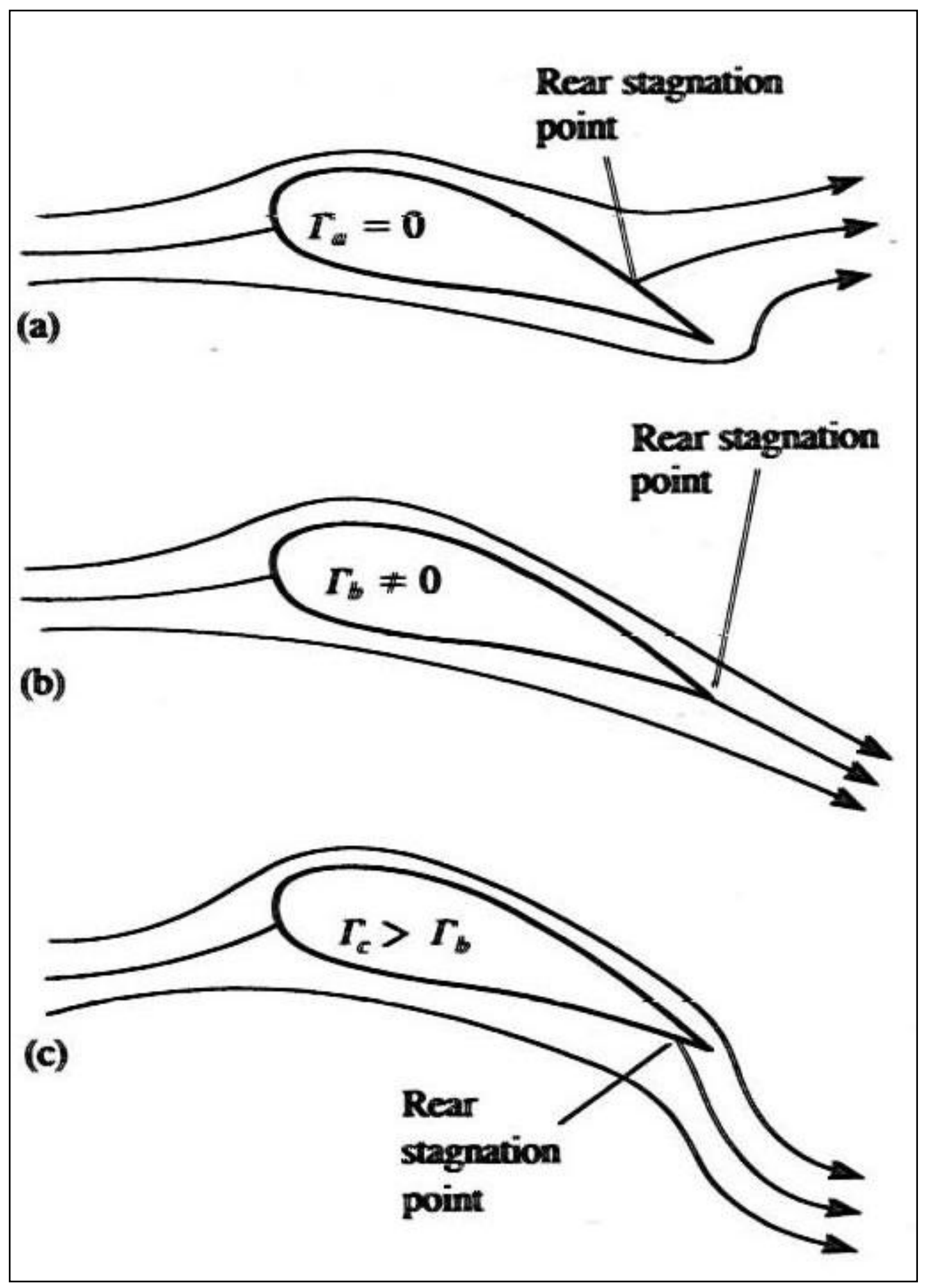

Figure 3. Potential Rear Stagnation Locations over Airfoils with Sharp Trailing Edges (Katz, 2001) 


\section{Circulation Control}

Circulation control as a high-lift device is traditionally used on the main wing of a fixedwing aircraft. This technology has been in the research and development phase for over sixty years, primarily for fixed-wing aircraft when the early models were referred to as "blown flaps." The first reported use of blowing slots to control lift is attributed to H. Hagedorn and P. Ruden, in 1938, who noticed an unaccountable increase in lift at high blowing rates during investigations into boundary layer control on a flap (Maskell and Spence, 1959).

Interest in active blowing systems increased with the advent of the turbojet engine, initially in Great Britain and France with a jet flap configuration. The flow of a fluid over curved surfaces has since been studied for a variety of applications. Circulation control works by increasing the near surface velocity of the airflow over the leading edge and/or the trailing edge of a specially designed aircraft wing using a series of blowing slots that eject high velocity jets of air (Kind, 1968). These wings have a rounded trailing edge, which eject the air tangentially through these slots inducing the Coandă Effect.

This phenomenon keeps the boundary layer jet attached to the wing surface longer than on a conventional wing and thus increases the lift generated on the wing surface due to the relaxation of the Kutta Condition for the rounded trailing edge. The circulation control airflow adds to the lift force through conventional airfoil lift production by altering the circulation of stream lines around the airfoil.

The main purpose of circulation control for fixed-wing aircraft is to increase the lifting force when large lifting forces and/or slow speeds are required, such as at take-off and landing. Wing flaps and slats are currently used during landing on almost all fixed-wing aircraft and on take-off by larger jets. While flaps and slats are effective in increasing lift, they do so with a penalty of increased friction drag. The benefit of the circulation control wing is that no extra 
friction drag is created from the movement of surfaces into the airflow around the wing and the lift coefficient is greatly increased (Loth, 1984). However, with the use of circulation control to increase the lift coefficient, there is a corresponding increase in the induced drag over the airfoil.

In past trials, a circular airfoil shape was used to analyze the potential fluid flow, (Theodorsen, 1931). The original methods are for frictionless, incompressible fluids, thus not truly valid for circulation control airfoils, especially those using active circulation control, due to the compression of air at the blowing slot. In 1975, more experimentation was done using the Theodorsen method in a potential flow analysis of circulation controlled airfoils (Gibbs, 1975). A Coandă Effect simulation was then conducted which under-predicted the decay of the maximum jet velocity (Churchill, 1992).

From previous experimentation, four main benefits were achieved by using an active circulation control method on fixed-wing aircraft to control moment augmentation (Ambrosiani, 1971 and Englar, 2005). These benefits are:

- Very small movement, or even non-moving, control surfaces are required,

- Lift augmentation can be achieved, independent of the airfoil angle-of-attack,

- Jet turning angle is no longer limited by physical jet exit angle/flap deflection angle, or

- Very high force augmentation can be achieved per unit blowing momentum input.

The pitching moment $\left(C_{m}\right)$ and center-of-pressure $\left(C_{p}\right)$ of the model are very important parameters when testing the stall angles in a wind tunnel environment. In 1970, two models were examined for the change in the pitching moment and center-of-pressure; the results concluded that at three different angles-of-attack, neither of these two parameters is significantly affected by a change in lift coefficient (Harness, 1970). 
Very few stall experimentations were conducted in past work with circulation control airfoils. However, an experiment was conducted on a 20.0 percent $t / c$, elliptical airfoil with 5.0 percent camber where the blowing coefficient $\left(C_{\mu}\right)$ was varied between 0.006 and 0.119 with a constant free-stream velocity of $100 \mathrm{~m} / \mathrm{sec}$ (Harness, 1970). Stall angles of this airfoil were increased from $9.0-14.0^{\circ}$ through this range of $C_{\mu}$. This stall study did not, however, take into account leading or trailing edge separation effects, only the effects of the entire suction surface of the airfoil. This airfoil's trailing edge was also unaltered, so the Kutta Condition still held true. It was found that adding a slight camber to the airfoils produced an increase in the lift augmentation ratio over the results found when testing a pure elliptical shaped airfoil.

\section{Inception and Early Circulation Control Research (1960's to 1970's)}

Circulation control has been implemented in various applications since its inception. Among these applications that have been studied in the past are high-lift fixed-wing aircraft, trailing edge, vertical/short take-off and landing aircraft (V/STOL), and anti-torque systems for rotorcraft.

In 1969, a study of 20.0 percent $t / c$, elliptical airfoils having circulation control systems were examined and tested under various angles-of-attack and free-stream velocity conditions. These airfoils showed most notably, that when the blowing coefficient is varied and angle-ofattack is held constant, a violent leading edge stall occurs, only correctable with a change in angle-of-attack (Williams, 1969). A second stall phenomenon occurred when the blowing coefficient was held fixed and the angle-of-attack was varied through a number of positions. This test also produced a trailing edge stall which was not as severe as the leading edge stall situation (Williams, 1969).

A series of testing scenarios of vertical/short take-off and landing vehicles was conducted in order to study low speed applications and wind tunnel capabilities. It was shown through this 
study that there is a minimum speed for testing, as determined by the wind tunnel geometry in which the flow seems to break down and the testing is no longer valid. Testing below this minimum tunnel speed produces a recirculation flow within the tunnel and is also shown to be a function of the downwash, configuration and size of the model (Rae, 1969).

In 1970, at West Virginia University ( $W V U$ ), a study was conducted on two circulation controlled cambered airfoils, Model A (20.0 percent $t / c$, elliptic, and 5.0 percent camber) and Model $B$ (20.0 percent $t / c$, elliptic, and 5.0 percent camber with a modified 5.80 percent $r / c$ trailing edge) in the hopes of proving that the addition of a high velocity jet of air ejected tangentially around the airfoil would produce a greater lifting force on the airfoil due to the Kutta-Joukowski Theorem. This theorem, expressed via Equation 11, states that the overall circulation around the airfoil $(\Gamma)$ is directly related to the lifting force $(L)$ when expressed in terms of the free-stream velocity $\left(V_{\infty}\right)$ and fluid density $\left(\rho_{\infty}\right)$ (Harness, 1970).

$$
L=\rho_{\infty} V_{\infty} \Gamma
$$

Equation 11

In the conducted studies, it was found that Model A produced a lift-to-drag ratio $(L / D)$ of 30.0 and Model $B$ produced a $L / D$ of 55.0 implying that circulation impacted the lift directly. This study also determined that the trailing edge shape is the major parameter that should be considered when dealing with a circulation controlled airfoil.

A second study conducted at West Virginia University used the aforementioned Model B, to determine how the rounded trailing edge of the airfoil impacts the addition of lifting forces on the aerodynamic body (Myer, 1972). Through this study, it was shown that the airfoil tested could attain a maximum lifting coefficient of 4.58 with a blowing coefficient of 0.17 . The author also claimed that the rounded trailing edge of 5.80 percent of the overall chord length was as optimal a design as could be used on this particular airfoil. The author further stated that the 
rounded trailing edge is the most important parameter when dealing with circulation control airfoils.

Adding to the studies conducted by Harness and Myer, a further experimentation of Model B was conducted in 1972 using the principle of pulsed flow to study the effect of turning the circulation controlled jets on and off in a repetitive manner (Holt, 1972). This investigation showed that providing pulsed air to feed the circulation control exit jets provided a 25.0 percent decrease in mass flow rate but still provided a 15.0 percent overall increase in lifting forces. This study showed that the feasibility of a circulation controlled airfoil for use in fixed-wing aircraft was not as dependent as previously thought on the addition of excess weight from mass regulation and pressurization equipment (Holt, 1972).

With the introduction of circulation control to rotary aircraft, a study conducted at $W V U$ was performed on a shrouded propeller in order to attempt to increase the static thrust of the propeller. The apparatus for this study used circulation control theory and the addition of blowing slots around the propeller shroud to change the effective velocity and pressure near the rotor (Ashworth, 1973). The study concluded that the circulation controlled shroud produced the effect of being a cambered shroud while circulation control was activated. Another result of this study showed that circulation control applied to this model successfully increased the overall thrust produced by the motor with increasing blowing coefficients.

Circulation control was applied to a helicopter rotor model in 1973, and was tested by means of installing the model into a wind tunnel. This "first generation" helicopter application demonstrated the use of circulation control over the entire rotor field as well as demonstrating rotor trim, lift augmentation, and maneuver moment generation through the use of cyclic blowing and circulation control over the model (Wilkerson, 1973). This was one of the first steps researched into physically applying circulation control principles to rotary powered aircraft. 
The second instance of attempting to apply circulation control principles to rotating bodies was made later in 1973. This study examined the inherent issues of using circulation control on a rotor, such as boundary layer separation due to an adverse pressure gradient, the inaccuracy of measurements, and the use of flow visualization methods. This study showed that the use of pressure taps on the model in order to measure lifting and drag forces experienced, is a much more accurate way of collecting necessary two-dimensional data than using a more conventional force balance method (Englar, 1973). Figure 4 (Englar, 1973) shows the typical distribution for the lifting forces attained when $C C$ is used on the trailing edge of an experimental airfoil. 


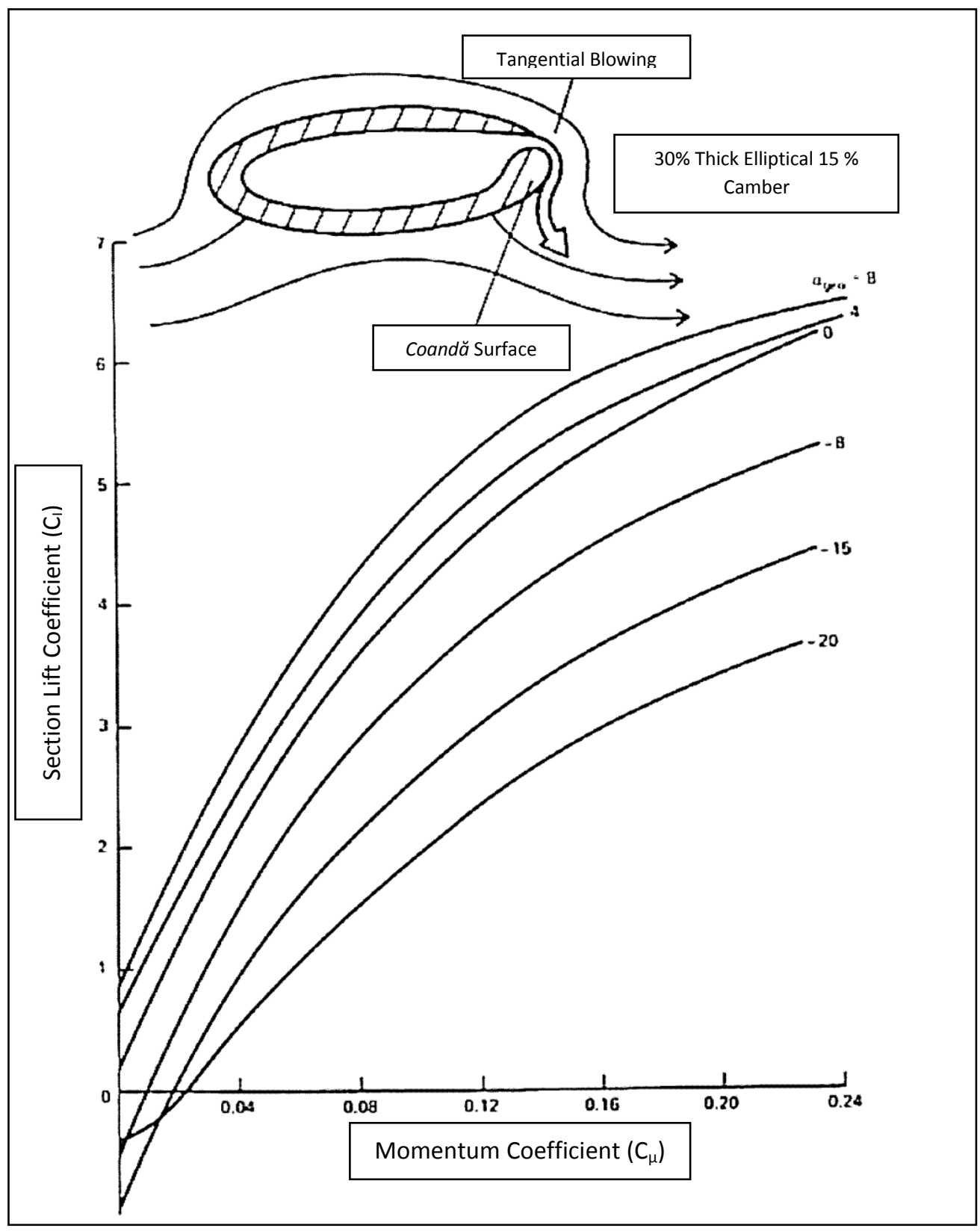

Figure 4. Typical Circulation Control Airfoil Lift Capability per Momentum Coefficient (Englar, 1973)

Again in 1975, two-dimensional circulation control wing sections were examined and found to produce maximum lifting coefficients of three times that of an unaltered airfoil, with a sharp trailing edge (Englar, 1975). These models were fabricated using existing National Advisory Committee for Aeronautics (NACA) shape profiles (NACA 66-210 and NACA 64A212), removing the sharp trailing edge and replacing one profile with a rotated flap configuration 
and the other profile with a rounded trailing edge of 0.875 inches. At various angles-of-attack and momentum coefficients, these test models produced lifting coefficients of 5.50. For the case of the rounded trailing edge model using a 0.20 blowing coefficient, one test model saw a lifting coefficient value as high as 5.95.

Another series of evaluations were performed on a 20.0 percent $t / c$ circulation control airfoil in 1975. In this testing scenario, an elliptic airfoil was tested at subsonic speeds in a two dimensional wind tunnel environment producing lift coefficients of 5.0 and above while the blowing coefficient was set at 0.241. Likewise, the lift-to-drag ratio $(L / D)$ of the activated circulation control model, reached a maximum value of 30.0 (Abramson, 1975).

In the mid 1970's, an analysis was done on arbitrary airfoils with the addition of circulation control abilities in order to predict the blowing slot characteristics for a given airfoil at known operating conditions. This study had a powerful effect on the study of circulation control in that it used potential flow, laminar boundary layer, turbulent boundary layer and turbulent wall jet theories applied together in order to approximate the geometry needed for the circulation control exit slots (Gibbs, 1975). The application of these aerodynamic theories to circulation control provided a basis on which to design a circulation controlled airfoil for a specific use without the need for several failed designs, including physical tests, and ultimately redesigns of the particular $C C$ airfoil.

Another rotary application of circulation control was conducted at the David W. Taylor Naval Ship Research and Development Center (NSRDC) in 1976, where CC hardware was applied to a high speed helicopter rotor and tested during reverse blowing conditions (i.e. while the rotor is in retreat in comparison to the free-stream velocity along the rotary path). This study employed a leading and trailing edge slotted airfoil in which circulation control was activated and seen to improve the overall performance of the helicopter during hover applications and 
through advance ratios of 4.0 (Reader, 1976). The design concept for the $C C$ rotor hub is shown below in Figure 5

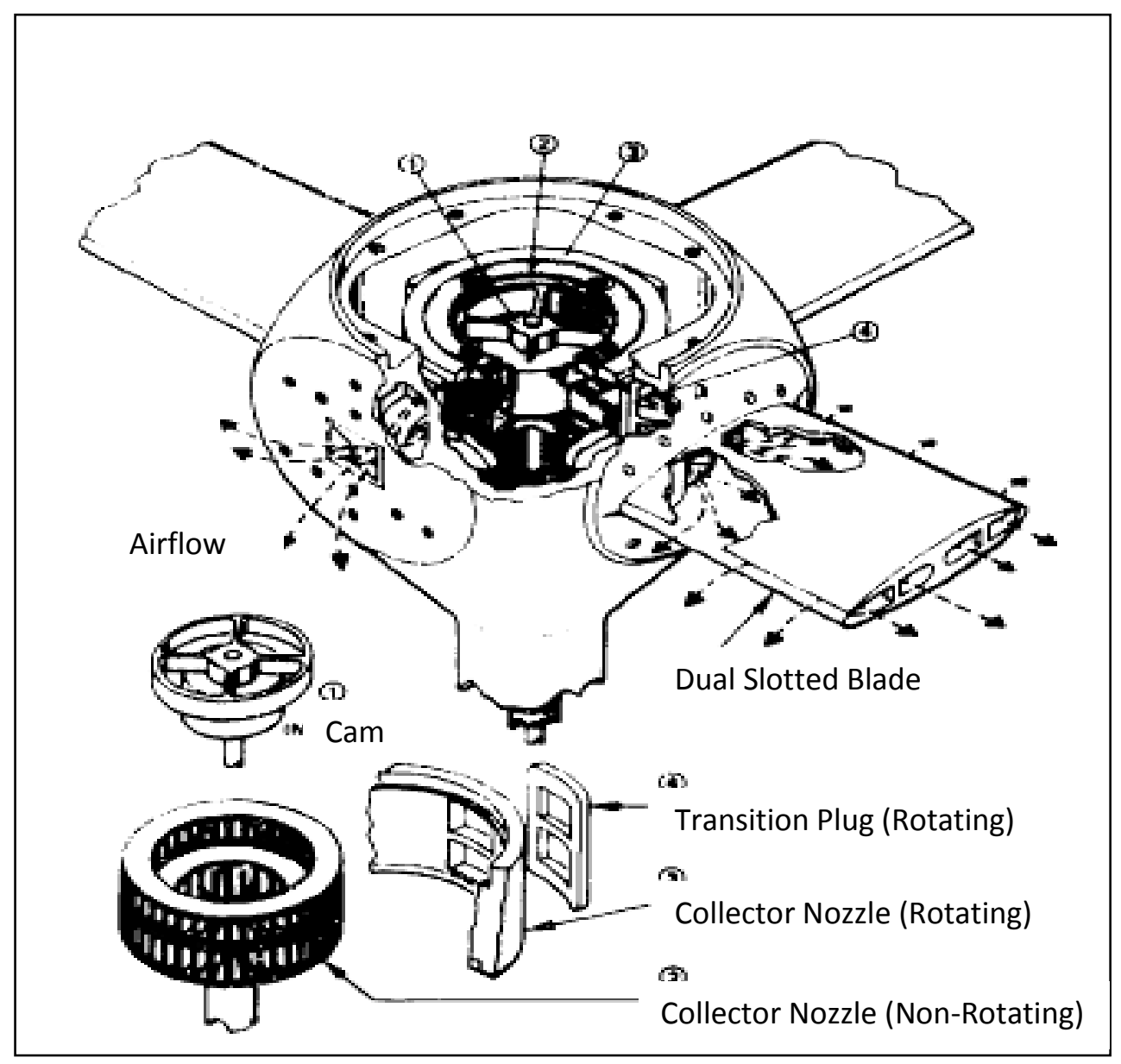

Figure 5. Reverse Blowing Circulation Controlled Rotor Hub (Reader, 1977)

This system also proved beneficial while flying through the critical advance ratio of 0.70 . Equation 12 shows the means of calculating the advance ratio of the rotor, using the free-stream velocity $\left(V_{\infty}\right)$ related to the rotational speed $(\Omega)$ and radius of the propeller $(r)$.

$$
\mu=\frac{V_{\infty}}{\Omega r}
$$

In the late 1970's circulation control was applied to the trailing edge of a fixed-wing aircraft, specifically on the A-6 flight demonstrator (Englar, 1979) in order to achieve larger lift 
coefficients at times of need, such as take-off and landing. A compressor was used to pressurize a NACA 64A008.4 (modified) model and exit pressure test cases were examined between 15.0 and $25.0 \mathrm{psf}(0.10-0.17 \mathrm{psi})$. In this testing, lift coefficients of 6.50 were found in twodimensional testing, and a three-dimensional lift coefficient was found to be a factor of 2.20 greater than without circulation control.

\section{Mid-Life of Circulation Control Research (1980's)}

As the development of circulation control technologies continued, research work and experimentation gained regard in the scientific community. With the new understanding of $C C$ technology, the applications of circulation control technologies began to expand from simply being an addition to existing aircraft to being seriously studied to improve performance on rotary powered aircraft. Also gaining interest were novel ways of producing the pressure for the $C C$ plenums located inside the airfoils, where it was shown that the addition of large scale pumping devices often negated the additional lifting forces achievable by adding circulation control.

After the introduction of circulation controlled rotor blades, in the 1970's, a study was designed to look at the differences in the noise levels of using such high-lift devices in place of conventional helicopter blades. The testing models included (1) the $C C R$ - circulation control rotor (ejects high velocity jets of air tangentially around a rounded trailing edge), (2) X-Wing (designed to stop and act as a fixed-wing at high forward speeds, and still employed circulation control technology on the upper surface) and, a (3) control scenario (a conventional helicopter rotor). In studies which used similar rotational speeds, advance ratios, and forward speeds, the $\mathrm{X}$-Wing rotor showed the most promise for eliminating blade/vortex noise as well as impulse noise, both common with conventional helicopter rotors. In testing the $C C R$, it was found that there was an abundance of broadband noise at high blowing velocities which produced high decibel levels during testing (Mosher, 1981). 
A study conducted at $W V U$ optimized the wall jet velocity used for circulation control applications in short take-off and landing aircraft. The information collected in this experiment showed that the addition of a Coandă surface and jet ejectors to a circulation control airfoil improved the $C C$ performance. This study also determined the need for a "stow-able" Coandă surface needed to be examined and implemented so that the $C C$ surface would be able to be taken out of the flow during cruise conditions when the high values for lift were not needed (Loth, 1984).

From 1984 to 1987, a series of experiments were conducted in conjunction with NSRDC (1984) and Lockheed Martin (1987), the first of which attempted to redirect the engine thrust of an aircraft to deflect the flow around a Coand $\breve{a}$ surface of the trailing edge of a wing. An above wing mounted propulsive device was used in this experiment. In a series of full-scale tests a deflection angle of 90 degrees was achieved, producing a lift coefficient of almost 6.0 (Englar, et al, 1984). This system also achieved lift augmentation equal to or in some cases greater than that of the multi-flap systems commonly used in commercial aircraft and offered this similar augmentation without the addition of weight and complexity of several moving parts. In 1987, the upper jet deflection apparatus was combined with a flow entraining system $(C C$ wing) which demonstrated the ability of the exhaust flow and the circulation control jet to work together to increase the lifting capability of the aircraft even further than the experiments conducted in 1984, yielding a $C_{L}$ upwards of 7.0 (Englar, 1987).

After completing the previous study of circulation control in attempting to optimize the jet velocity, the next step in the process of studying this technology at $W V U$ was to analyze the thrust savings that the addition of circulation control could provide to aircraft using blown jet high-lift wings. It was found that high-lift devices such as circulation control and boundary layer control apparatuses have a distinct upper lifting coefficient limit that can be achieved. Any 
attempt to exceed this optimum lifting coefficient, as in high speed applications, would cause rapid deterioration of the boundary layer control objectives (Loth, 1987).

\section{Newest and Ongoing Circulation Control Research (1990's - 2000's)}

In the most recent historical time period, circulation control techniques are shown to have become more predictable, as well as, they are shown to be productive in several previously under-researched scenarios, including water/other fluid mediums, vehicular uses other than aircraft (i.e. tractor trailers and automobiles), and wind turbines. It can also be shown that the future uses of circulation control are expansive, in comparison to the original ideas for the uses of this technology. Further research in this period includes the ability to reduce the amount of additional hardware to the system and attempts to enhance the control of the system, or increase its effectiveness.

In an assessment using a stopped rotor in conjunction with circulation control in the early 1990 's, a study was performed to analyze and predict the effects of the addition of circulation control benefits. This study produced a software tool which predicts trade-offs in sizing appropriate stopped rotor systems based on horsepower, forward flight speed, mass flow requirements as well as predicting some of the transition performance while the aircraft travels at high speeds (Tai, et al. 1996).

In 1996, at the Georgia Institute of Technology, a new application of circulation control was envisioned. It was hypothesized that because the modern ground vehicle, automobiles and long haul tractor trailers, are influenced greatly by vortex shedding and separated flow fields, the addition of circulation control technologies might positively impact the fuel consumption and efficiency of these vehicles. The experimentation conducted, on a generic fiberglass automobile model, showed that a 35.0 percent reduction in overall drag in comparison to an already streamlined vehicle without circulation control. Another facet of this research concluded that the 
application of lower surface blowing, as opposed to upper surface activation can provide an increase in down-force on the vehicle as well as reducing the instability of the automobile while in a yawing motion, thus showing that active blowing can be used to restore the lateral stability of the model (Englar, et al. 1996).

Although circulation control was always envisioned to be a productive system for enhancing lift in any fluid medium, the vast majority of studies up until the mid 1990's examined the system within the constraints of using air as the pressurized fluid medium. In 2004, a water tunnel experiment was conducted in the Large Cavitation Chamber, located in Memphis, TN. This study used a circulation controlled wing having a 20.0 percent $t / c$ elliptic cross-section, an aspect ratio of 2.0, and dual upper and lower jet exit slots. This model also employed a slight amount of taper, overall, 0.76 across the span. Unlike the previous tests which used air as the pressurization medium, this model provided a visual representation of the behavior of the exit jet at various angles-of-attack and plenum pressures (Rogers, 2004).

One unique finding of this particular research was the fact that when cavitation was forced to occur on the Coandă surface, there was no abrupt stall of the airfoil. The model showed signs of a slow, even decrease in the lifting forces. This shows that the reduction of the pressure around the exit jet and Coandă surface while decreasing the lifting force as expected, will not become detrimental, and will still allow the wing, propeller, or airfoil to produce a lifting force, even at conditions of higher speed, greater depth/altitude, and increased pressure. Also shown in this study was the possibility of producing a positive lifting force at a geometric pitch angle of negative 40.0 degrees. In comparison to ship surfaces of the same geometry, this particular $C C$ enhanced model produced a lifting coefficient of 3.0, nearly double that of its unmodified counterpart. 
In 2004, again at the Naval Surface Warfare Center (NSWC), an elliptic airfoil section of 1.0 percent camber, and 0.17 thickness-to-chord ratio was evaluated to examine the low speed characteristics of performance. This model was tested with both upper and lower $C C$ exit slots and at a zero angle-of-attack so as to experiment with the blowing efficiency of the system. This model produced a positive 3.60 lift augmentation coefficient during employment of the upper slot exit jet, while the lower slot produced a negative 4.0 lift coefficient. This result showed that using a negative effective camber on a circulation control airfoil produces a greater lift augmentation ratio and lifting force (Abramson, 2004).

Similar studies to that of the fiberglass model automobile studied in 1996, were expanded in 2005, however, the circulation control system was directly applied to a tractor trailer and examined in real highway conditions. With the use of heavy vehicles to transport goods, and the fact that a typical tractor trailer averages 175,000 miles a year, fuel costs alone average upwards of \$40,000 each year (Englar, 2006). The application of circulation control technologies to these types of vehicles, although offering only a small benefit in comparison to traditional $C C$ applications, can still be quite meaningful. The idea for this technology was to use $C C$ slots at the rear end of the trailer and try to "pull" the airflow around the truck in order to reduce the overall drag (Figure 6). 


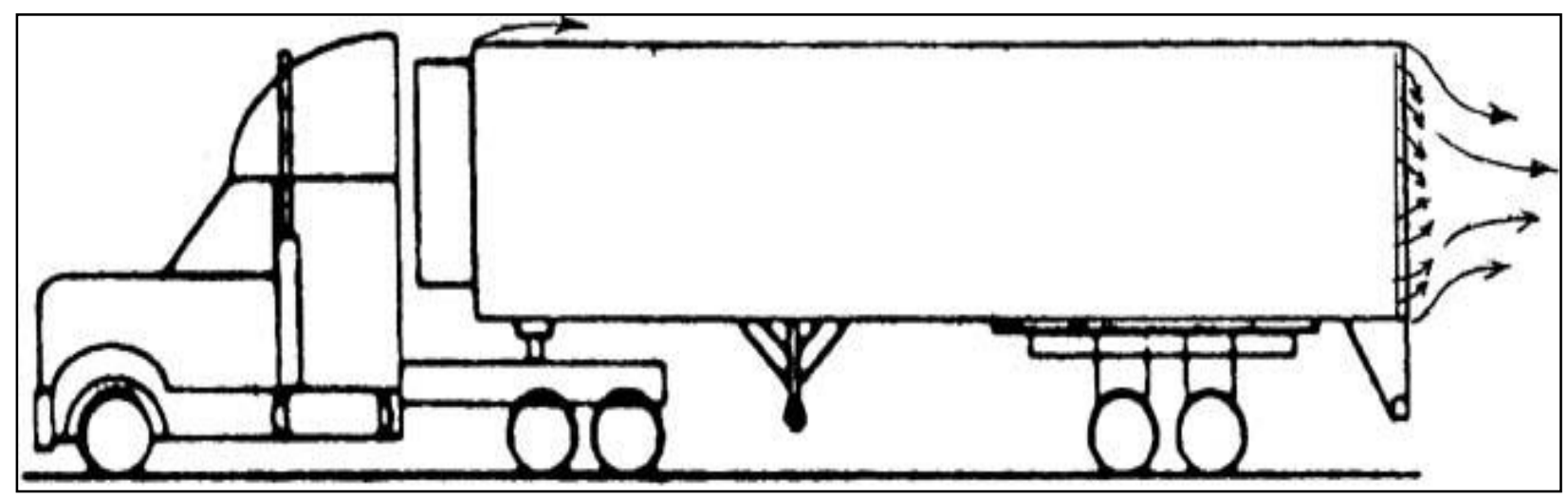

Figure 6. Concept Drawing of $C C$ Controlled Tractor Trailer (Englar, 2006)

Preliminary testing of this type of application was conducted in a wind tunnel environment in order to get an idea of design feasibility. The next step in the testing process lead to an actual tractor trailer being retro-fitted with appropriate circulation control hardware along the length and around the back door of the trailer. A second replica tractor trailer was also examined in order to produce a baseline comparison test. The results showed that activation of certain blowing slots can reduce drag (in the wind tunnel model) up to 84.0 percent by preventing flow separation at the rear end of the vehicle. It was also noted that the activation of top blowing slots only can increase the lifting force experienced by the wheels of the truck and thus increase its fuel economy. Several ideas for the supply of circulation control pressurized air, without the addition of heavy, complicated after-market parts, include a turbo/super charger onboard or already existing auxiliary engines (such as cabin generators and refrigeration motors). The test truck which was fitted with a rounded edge, blowing slots and wheel shrouds can be seen in Figure 7. 


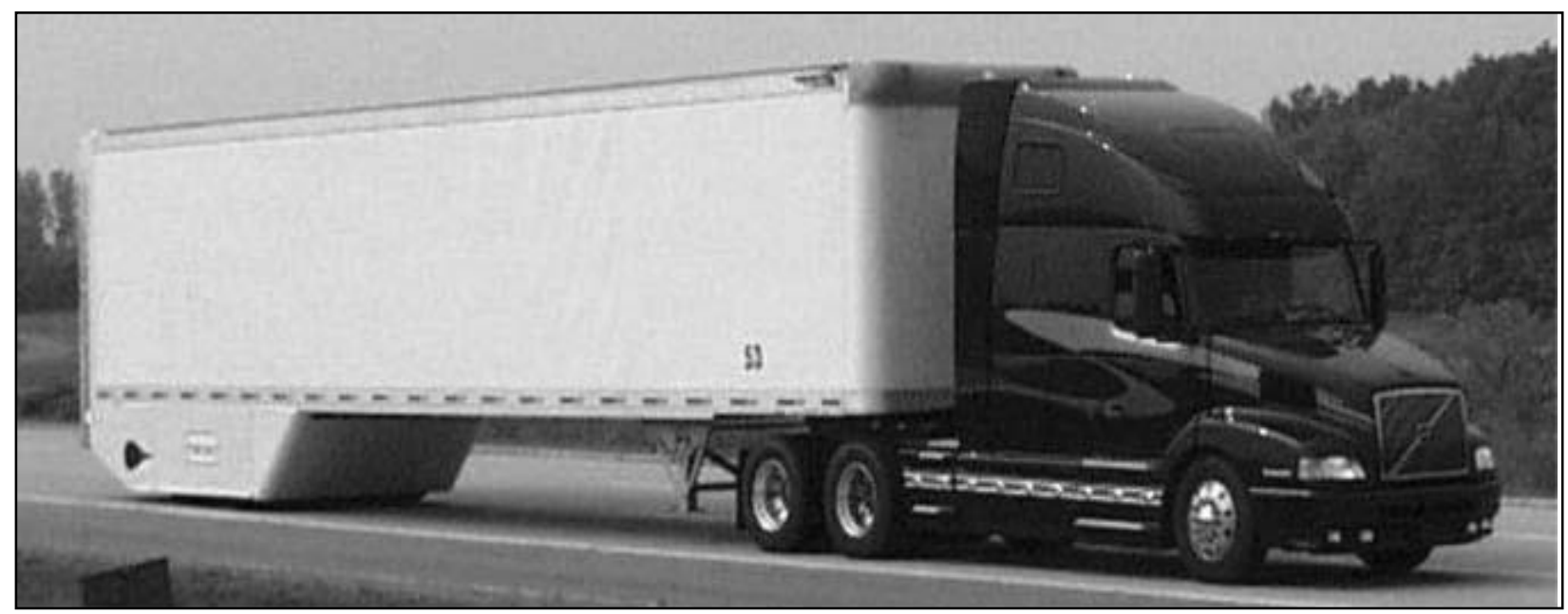

Figure 7. Circulation Control Tractor Trailier being Road-Tested (Englar, 2006)

In 2006, a rapid predictive method was studied for the implementation of circulation control techniques at the Georgia Institute of Technology. In this study, two-dimensional circulation control performance calculations were made using the Navier-Stokes Equations for fluid flow (Equation 7, Equation 8, Equation 9, and Equation 10 above). Coupled with a computer code to calculate circulation control airfoil characteristics, this method set out to predict the interaction between variables involved with circulation control systems. Using flow visualization, quantitative, and qualitative methods, it was possible to compare the aerodynamic forces achievable by adding circulation control. Although the particular experiment is only useful for selected ranges of variables such as pressure and density, the overall modeling can be used to predict the effect of adding circulation control to a model operating at any range of variables, so long as these variables are all called out at the beginning of the simulation (Naqvi, 2006)

In the interest of forwarding the study of circulation control effects in alternate fluid mediums, an experiment was conducted at the University of Strathclyde, England. Here they implemented a modified marine propeller duct with circulation control capabilities. The study focused on using a propeller duct to eliminate the use of conventional control planes on 
autonomous underwater vehicles (AUV's). The use of this altered propeller duct showed an effective increase of the maneuvering force produced, of about 600 percent, and an increase in the efficiency of the overall ability to maneuver the craft by 9.50 percent over the ability of the conventional lifting surfaces (Ward, 2006). This study further showed the availability of a $C C$ application to a wider array of uses, particularly uses involving hydrodynamics and other water applications.

In 2006, at West Virginia University, a conceptual design of a new helicopter, with the addition of circulation control blowing slots, was studied, in the hopes of using this technology to reduce or eliminate the need for a swashplate design. This application used $C C$ slots on the helicopter blades to increase the lifting forces of the aerodynamic surfaces, thus increasing the payload of the system while eliminating the need to articulate the blade angle continuously throughout the rotation of the blade. Although this design is still in the development stages, it suggests that with the use of $C C$ sciences, the reliance on the major limiting factor and failure point of helicopters, the swashplate, can be reduced or even eliminated (Angle, et al. 2006).

Further research work was conducted at NSWC in 2006, on the dual slotted $C C$ wing, in the Large Cavitation Channel in Memphis, TN. In order to measure the performance of the elliptic airfoil, a six component load cell and laser-doppler velocimetry data sets, were taken in the wake of the airfoil (Donnelly, et al. 2006). An extensive number of tests were run in order to produce characteristic loading of the model due to angle-of-attack, slot exit velocity, and cavitation impact. With the results of this test documented, a computational model was envisioned to be built, simulated, and compared to the data found in testing in order to get a general understanding of the effect of $C C$ on several different cross-sectional hydrodynamic surfaces. 
In another study of a unique implementation of circulation control sciences, active (leading edge and trailing edge slots and exit jets) and passive (Gurney Flap) $C C$ systems were applied to a horizontal axis wind turbine $(H A W T)$. In the application of this system to a leading edge ejection slot model, shown in Figure 8, the results showed that at high wind speeds the enhancement with circulation control techniques breaks down and is no longer applicable. When the passive Gurney Flap system, as well as the trailing edge activated slot was employed on the model, a circulation increase around the airfoil was observed with a net increase in power generation capability in comparison to the unaltered model. The activation of trailing edge blowing slots (Figure 8) exhibited a larger aerodynamic force output as well as a larger power output available than the model employing a passive Gurney flap system. However, the application of the gurney flap without the need for an external pumping system gave it versatility in applications where no additional weight or added complex hardware is allowable (Tongchitpakdee, 2007). This selected study, although focusing on HAWT configurations, could also be applied to vertical axis wind turbines (VAWT) as well as water turbines and coaxial, multi-stage compressors. 


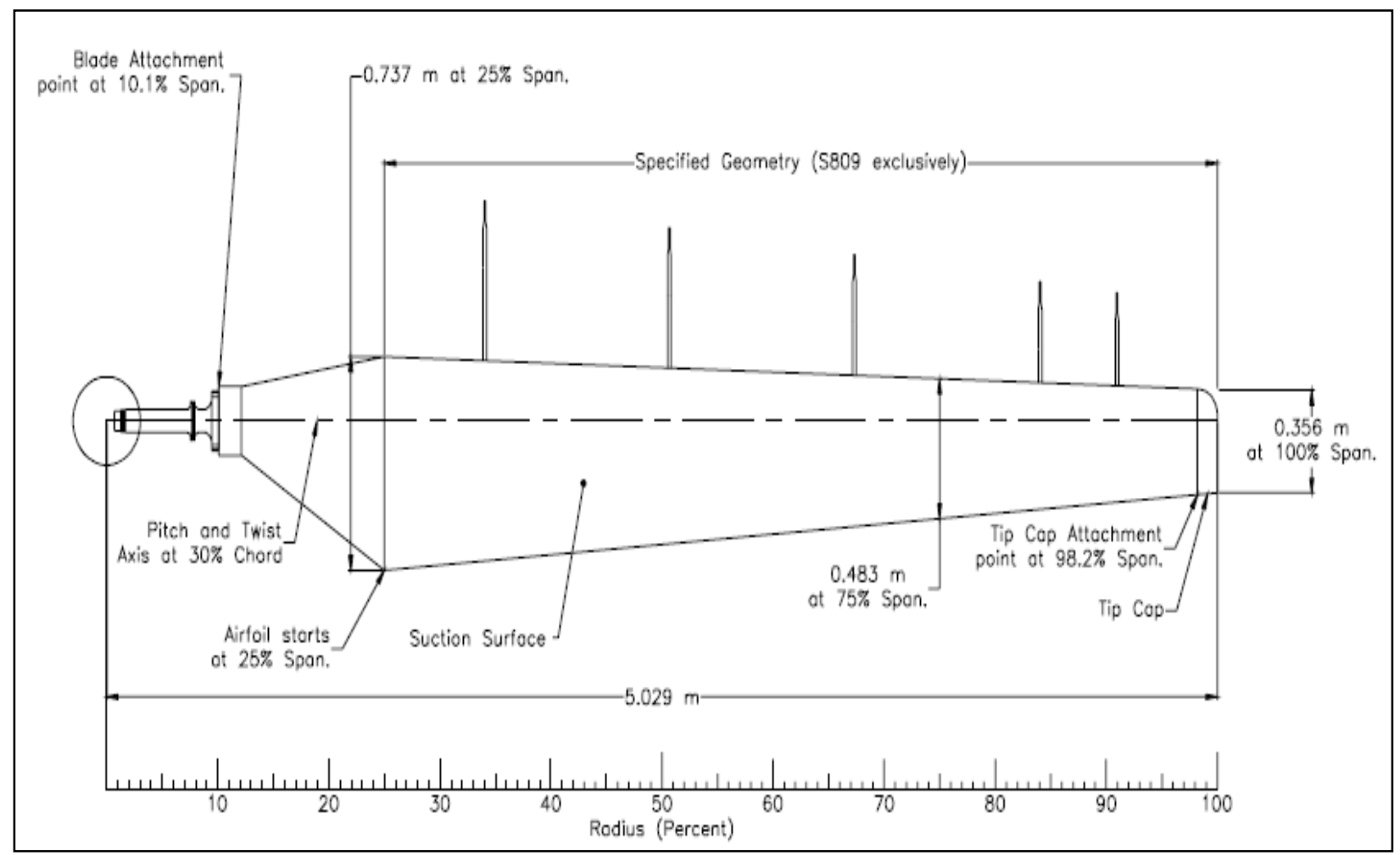

Figure 8. CC Wind Turbine Blade Planfom Dimensions (Tongchitpakdee, 2007)

A more recent testing scenario, of near surface actuation, circulation control for use on a rotorcraft main rotor was conducted at West Virginia University (Angle, 2008). Again, in this scenario, an external compressor was used to pressurize the plenums of the experimental model, shown in Figure 9, to 10.0-15.0 psig, resulting in a slot exit velocity of $1000 \mathrm{ft} / \mathrm{s}$. The circulation control velocity has a bearing on several factors including slot height, slot length and plenum pressure. As with Englar's work, this study produced results which showed a lift coefficient in excess of 5.0 is achievable. Also in this work, the response times of a circulation control model of a 10:1 $\mathrm{h} / \mathrm{c}$ ratio elliptical airfoil was determined to be $55.0-60.0 \mathrm{~ms}$. 


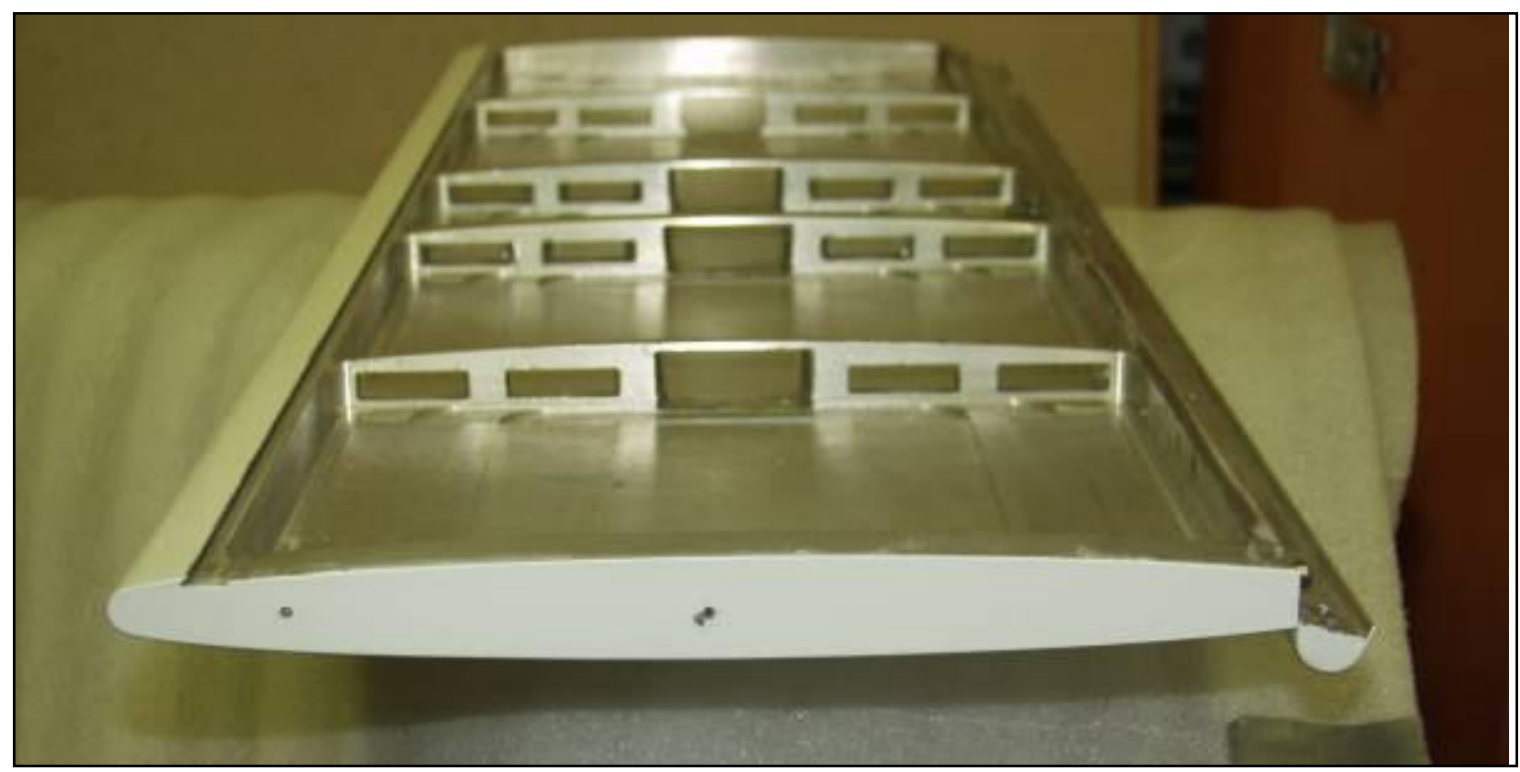

Figure 9. Circulation Control Plenum and Rib Spacing of $\mathrm{CCHB}$ Model (Angle, 2008)

In 2009, preliminary studies were conducted at West Virginia University to add circulation control technology to a vertical axis wind turbine (VAWT). Selection of a circulation control airfoil was completed to be used on an H-Type VAWT. Performance predictions were studied after adding circulation control to a computationally simulated vortex generation model. Blowing coefficients of 0.0 percent, 1.0 percent, and 10.0 percent were studied over a range of solidity ratios, 0.01-0.4. It was found that the overall power output performance of the turbine would be increased 24.0 percent at a blowing coefficient of 10.0 percent (Wilhelm, et al., 2009).

This work continued through the implementation of a momentum model of the wind turbine in order to begin development of the control algorithms needed to use circulation control most efficiently around the wind turbine's blade path. The initial two-dimensional version of the model proved successful in modeling the interaction and effects of the turbine and its wake region (Wilhelm, et al., 2009). Below, in Figure 10, the model of the VAWT is shown and most significantly exhibits the vortex production at the 90 and 270 degree position of the rotation, as well as the wake confinement to the original diameter of the turbine. 


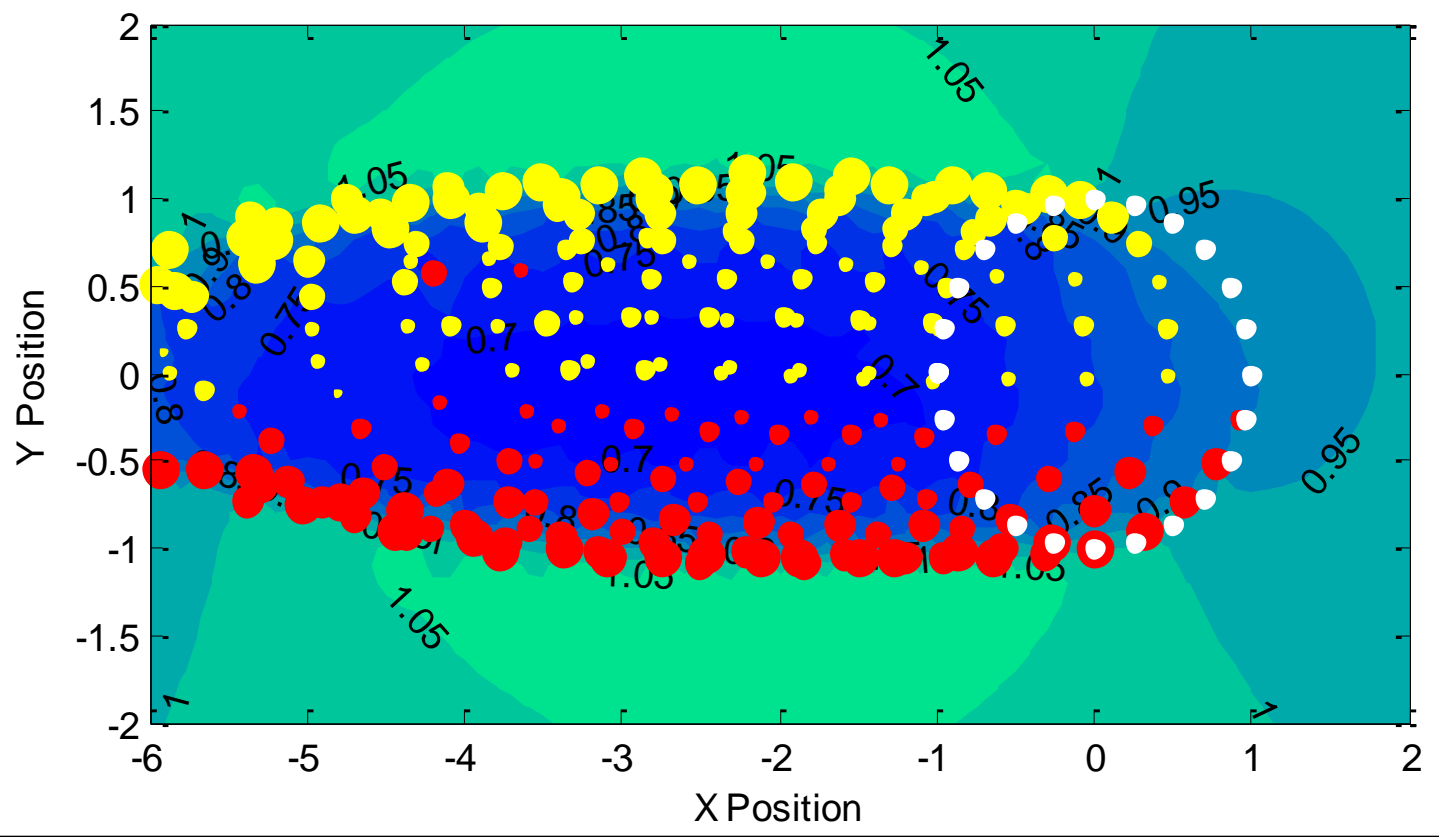

Figure 10. Vortex Model Velocity Field with Vortices and Blade Positions (Wilhelm, et al., 2009)

\section{Summary of Circulation Control}

High-lift and boundary layer augmentation devices have progressed through their history as mechanically deployable and stow-able surfaces to air entrainment and pressurized systems used to give the appearance of an aerodynamic surface without the need for heavy mechanical moving systems. Some of these systems have been used in the past as merely lift generation systems employed at specific times when most needed, and others have been used in conjunction with other aerodynamic systems to provide both an increase in lifting forces and/or a decrease in drag improving the overall efficiency of the vehicle. With the interest in fuel efficiency and increasing overall vehicle performance to become more energy independent, there is renewed vigor in research into high-lift and high-efficiency systems for addition to current aircraft, watercraft, and even ground transportation vehicles. 


\section{Propellers and Rotating Bodies}

The concept of a screw propeller is not new. In 950 BC, the Egyptians used a screw-like device for irrigation purposes. Archimedes (287-212 BC), the first scientist whose work had a lasting effect on ship propulsion is credited with the invention of the screw (Carlton, 2007). His screw pump, created to pump out flooded ships and for supplying water to irrigation ditches, was the forerunner of the screw propeller. Archimedes' screw applied the spiral movement in a determined space in order to automatically lift the water to a new location. Later, Leonardo da Vinci adopted the same principle to drive his theoretical helicopter (Figure 11) in which his sketches show a large canvas airscrew overhead of the cockpit of his flying machine (Vezzosi, 1997). The same principle found in early propellers can also be seen in "sculling."

The skill of "sculling" is a time honored talent used to operate Venetian gondolas. The rower, using a single blade, moves the blade through the water in a gentle arc, taking care to present the blade at the most effective angle to the water. In today's world the simplest activity of rowing a canoe or kayak can be harkened back to early propeller principles, with what is referred to as the " $\mathrm{j}$-stroke."

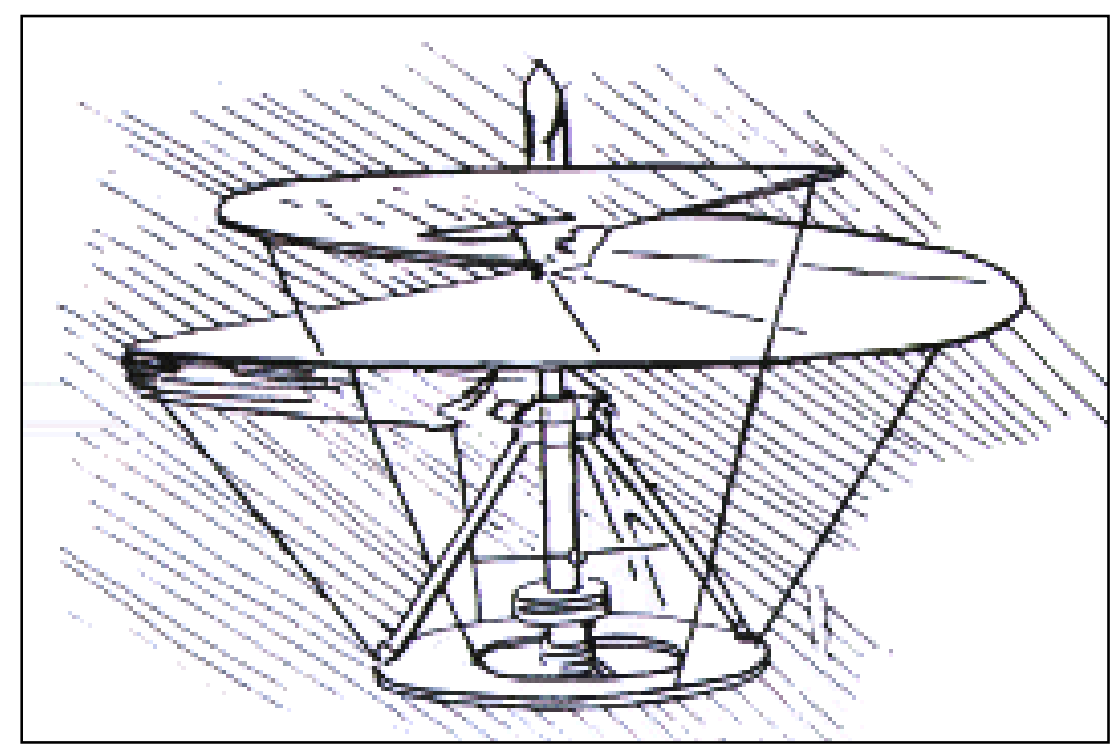

Figure 11. Leonardo da Vinci's Helicopter Design (Vezzosi, 1997) 
In 1784, J. P. Paucton proposed a gyrocopter-like aircraft using similar screws for both lift and propulsion, and at about the same time, James Watt proposed using screws to propel boats, although he did not use them for his steam engines. This was not Watt's own invention, though; Toogood and Hays had patented it a century earlier, and it had become a common use as a means of propelling boats since that time (Seif, 2004). By 1827, Austrian-Czech constructor Josef Ressel had invented a screw propeller which had multiple blades fastened around a conical base. This new method of propulsion allowed steam ships to travel at much greater speeds without using sails thereby making ocean travel faster.

Propellers remained extremely inefficient and rarely used until 1841, when Francis Pettit Smith discovered a new way of building propellers (US Pat. 2353, 1841). Up to that time, propellers were literally screws, of considerable length. But during the testing of a boat propelled by one, the screw snapped off, leaving a fragment shaped much like a modern boat propeller. The boat was found to have moved faster with the broken propeller than it did with the entire screw assembly intact. At about the same time, Frédéric Sauvage and John Ericsson (US Patent 4,181, 1845) applied for patents similar, although less efficient shortened-screw propellers, leading to a permanent controversy as to who is the official inventor among those three men. Ericsson became widely famous when he built the Monitor, a US Federal armored battleship that in 1862 fought the Confederate States' vessel Virginia in an American Civil War sea battle.

Several propellers have been designed and employed over the years, including: constant speed propellers, variable pitch propellers, constant pitch propellers, contra-rotating, dual shaft, high speed and low speed varieties, all employing different planforms to maximize efficiency, or tailor the flight characteristics to a specific use. Typically, propellers can have anywhere from two to eight blades depending on the work schedule. 
The constant pitch propeller, commonly used in hobby and small aircraft applications, uses a solid propeller to gain a maximum amount of thrust and greatest efficiency rating at cruise speeds. It is generally the most simplistic type of propeller, mainly because, the same planform and pitch is used throughout the rest of the flight envelope at the cost of some efficiency. This reduces the amount of moving parts that are associated with the propeller and allow for easy maintenance of the aircraft. Conversely, a variable pitch propeller uses feedback information from the flight to change the pitch of the propeller and increase the efficiency to the maximum amount. These propellers use servo-like mechanics under the nose cone to move the propeller plane while in use.

In the case of a constant speed propeller, similar flight feedback is used, however, the pilot chooses a specific airspeed to fly at and the propeller pitch mechanisms control the propeller planes to provide a constant forward speed at any altitude. Again, sometimes this constant velocity comes at the sacrifice of efficiency of the system. Figure 12 shows some visual representation of a few other propeller blade planforms, including low-speed (a), high-speed (b), and transonic (c) varieties. 


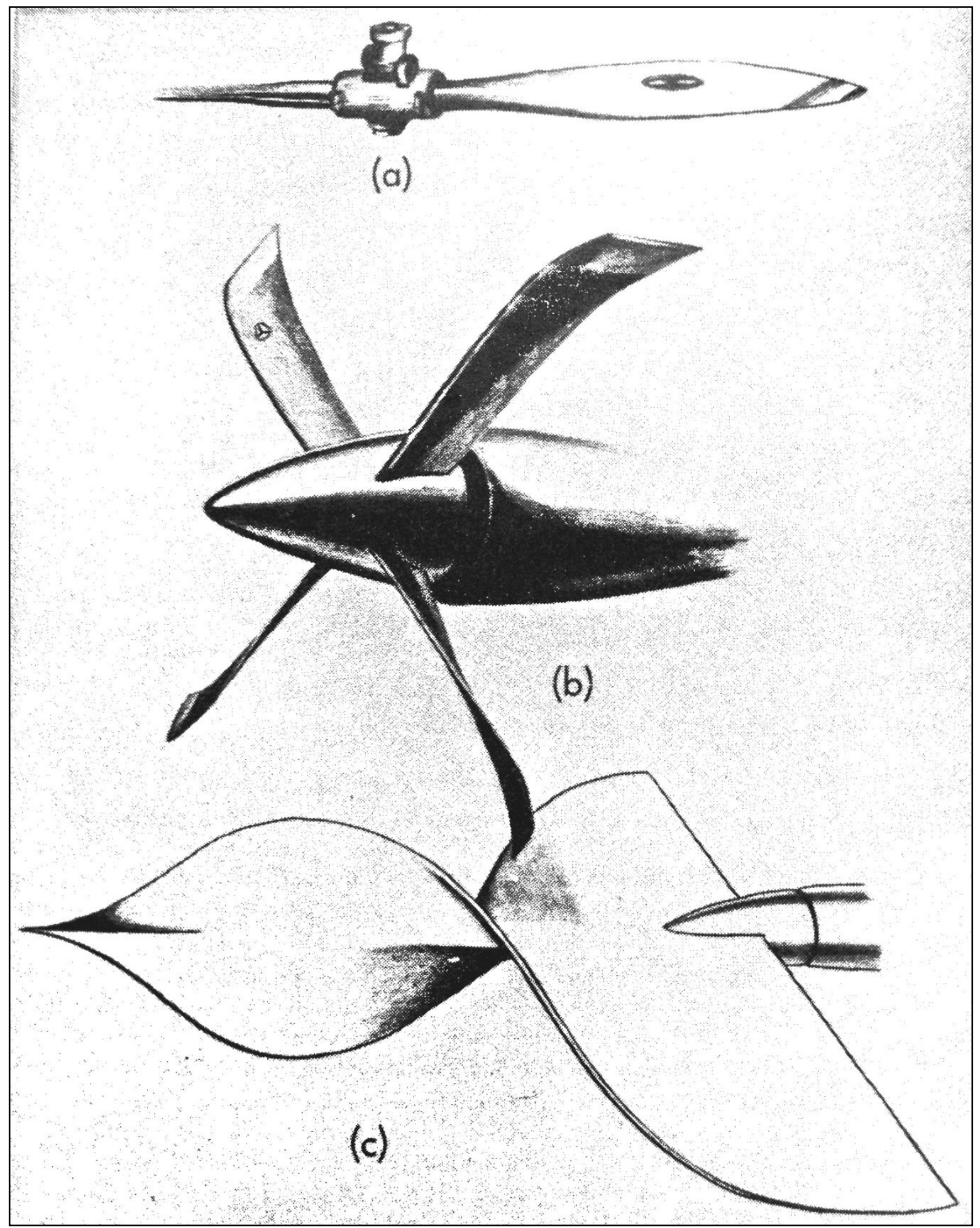

Figure 12. Different Propeller Planforms (Dommasch, Sherby, and Connolly, 1961)

In the second half of the nineteenth century, several propeller theories were developed.

The momentum theory or disk actuator theory (Figure 13), a theory describing a mathematical 
model of an ideal propeller, was developed by W.J.M. Rankine (1865), Alfred George Greenhill (1888) and R.E. Froude (1889). The propeller is modeled as an infinitely thin disc, inducing a constant velocity along the axis of rotation. This disc creates a flow around the propeller. Under certain mathematical premises of the fluid, there can be extracted a mathematical connection between power, radius of the propeller, torque and induced velocity. The omission of frictional forces made this a simplistic view of the dynamics of a propeller (Reissner, 1942).

This simplest way of examining the propeller is used mainly to visualize the propeller and the reactionary forces it produces without having to analyze very detailed information and angular geometries of the propeller. This method assumes that the measured velocity in front of the propeller plane and the measure velocity behind the propeller plane, is directly correlated to the thrust output of the propeller disk with the application of a simplified version of Bernoulli's Equation (Equation 13) and Newton's Second Law (Equation 14) (Dommasch, Sherby, and Connolly, 1961). The application of these two relationships can estimate the thrust produced by a propeller at a given operational environment as to well as estimate the flow characteristic velocities through the propeller plane itself.

$$
\begin{array}{cc}
T=A \Delta P & \text { Equation } 13 \\
T=m a=\frac{m d V}{d t} & \text { Equation } 14
\end{array}
$$




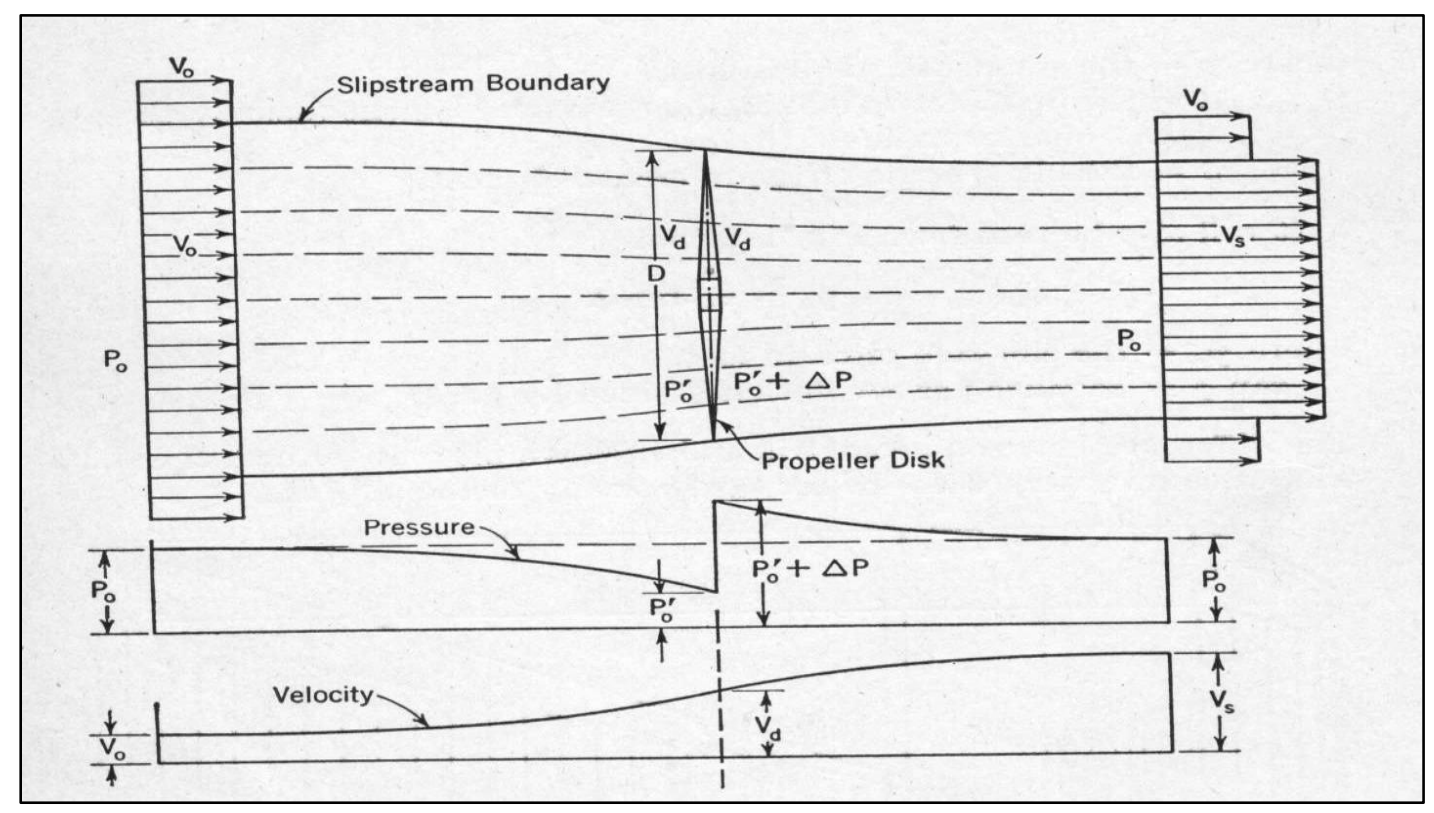

Figure 13. Physical Representation of Disk Actuator Theory (Nelson, 1944 )

Later in the nineteenth century, blade element theory, a mathematical process originally designed by William Froude (1878), David W. Taylor (1893) and Stefan Drzewiecki (1892), was first conceived to determine the behavior of propellers. This theory involves breaking an airfoil down into several small parts and then determining the forces on those smaller sections individually. These separate forces are then converted into accelerations, which can be integrated into velocities and positions (Glauert, 1943). This less simplistic view of estimating the parameters involved with the analysis of a propeller is the blade element theory (BET). According to historical sources, the theory involves looking at the propeller as a rotating airfoil so that each point on the airfoil follows a helical path throughout its rotation, as seen in Figure 14, below (Dommasch, Sherby, and Connolly, 1961). 


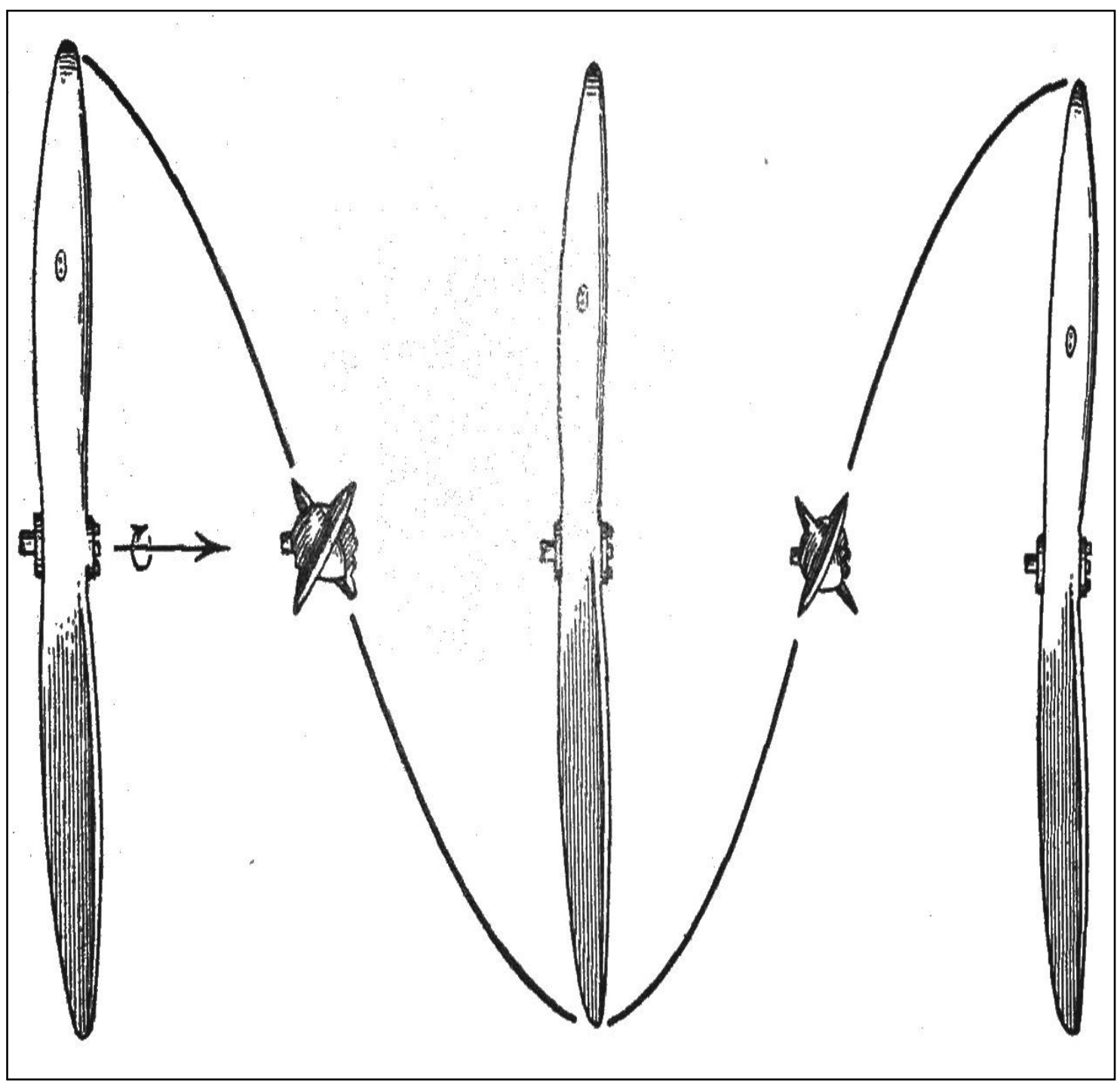

Figure 14. Generic Rotational Path of a Propeller in Two Dimensions (Dommasch, et. al, 1961)

This simplifies to a series of angular measurements throughout the radial locations of the blade as well as throughout its rotational path with relation to the free-stream velocity (Dommasch, Sherby, and Connolly, 1961). Below, in Figure 15, it can be seen that the angle-ofattack of the airfoil $(\alpha)$ changes throughout the rotation of the blade based on the sweep and pitch angle of the propeller plane $(\beta)$. This method more accurately estimates the impact of the propeller on the surrounding air with the correct airfoil cross-sectional data input into the 
programming. With the ability to know the geometry at a seemingly infinite number of blade locations across the radial span of the propeller blade, the accuracy of this method can be enhanced further and further. Generally, blade elements taken at each 10.0 percent of the radial distance traversing out to the tip of the propeller is sufficient enough to estimate accurately (Nelson, 1944).

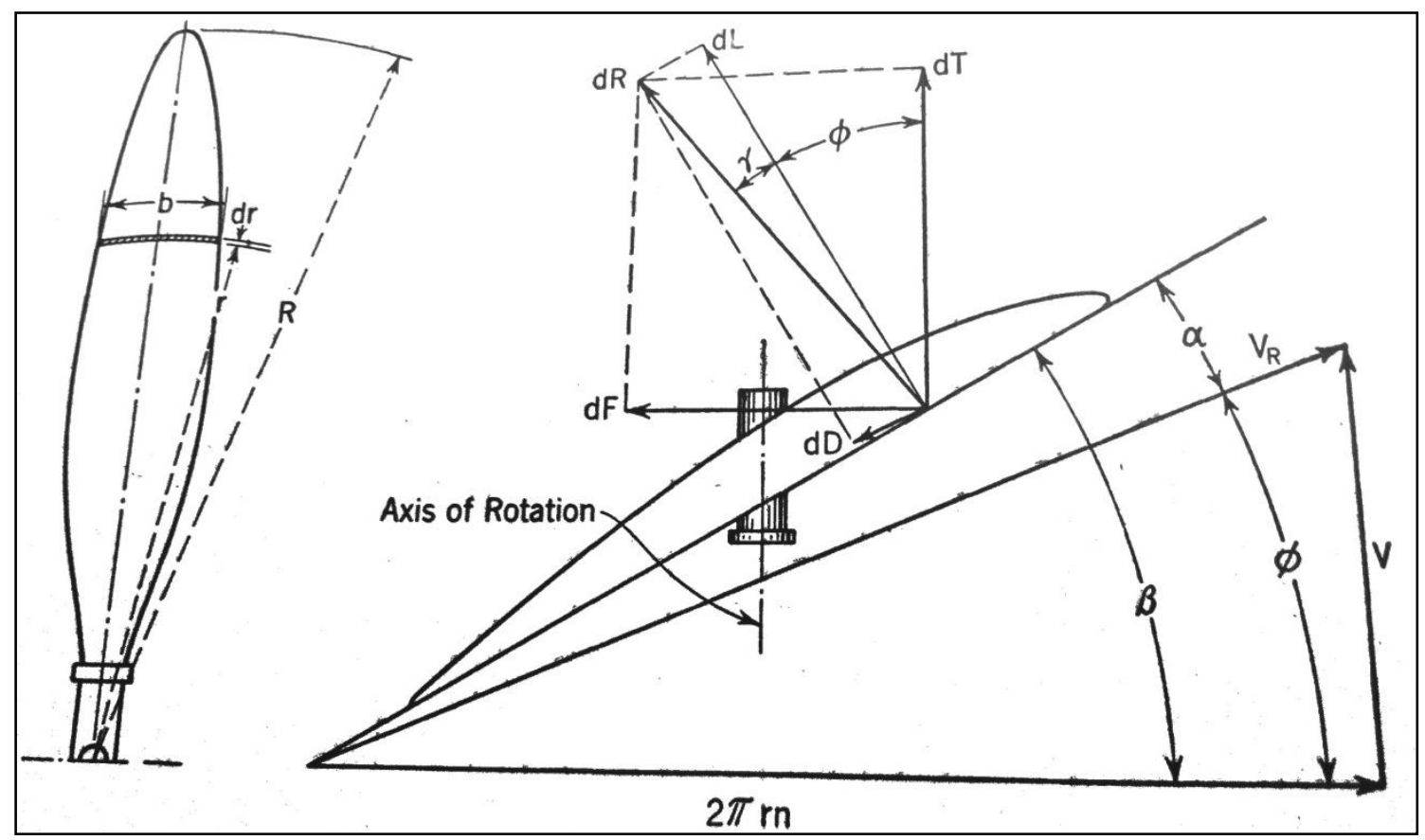

Figure 15. Blade Element Angular Relationships (Nelson, 1944)

The twisted airfoil shape of modern aircraft propellers was pioneered by the Wright brothers in the early years of the twentieth century. While both the blade element theory and the momentum theory had their supporters, the Wright brothers were able to combine both theories. They found that a propeller is essentially the same as a wing and so they were able to use data collated from their earlier wind tunnel experiments on wings. They also found that the relative angle-of-attack from the forward movement of the aircraft was different for all points along the length of the blade, thus it was necessary to introduce a twist along its length (Figure 16). Their 
original propeller blades are only about 5.0 percent less efficient than the modern equivalent, some 100 years later (Ash, et al., 2003).

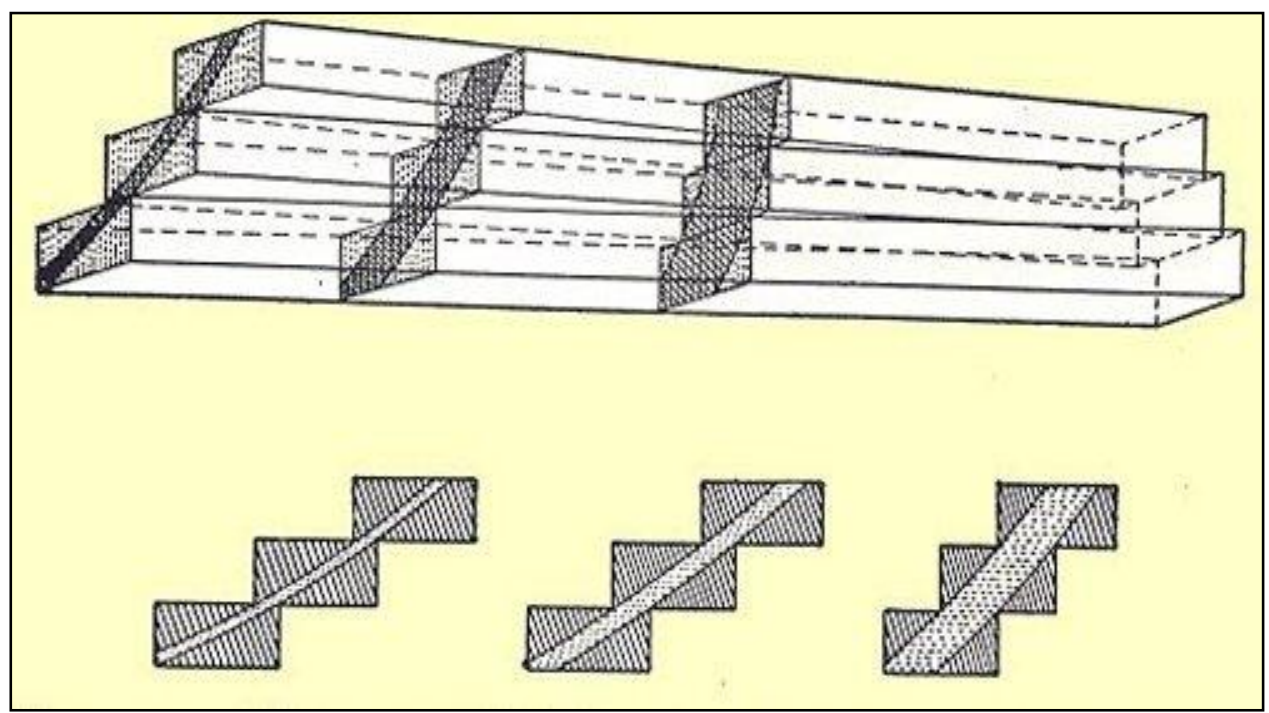

Figure 16. How the Wright Brother's Propeller was Cut from Three Planks (Ash, 2003)

Several newer methods of producing propeller blades are shown in Figure 17 (Nelson, 1944), below, with the most prevalent method in the recent days being made of some form of hardwood with reinforcements or made of a solid aluminum machined or in rare occasions an extruded blade.

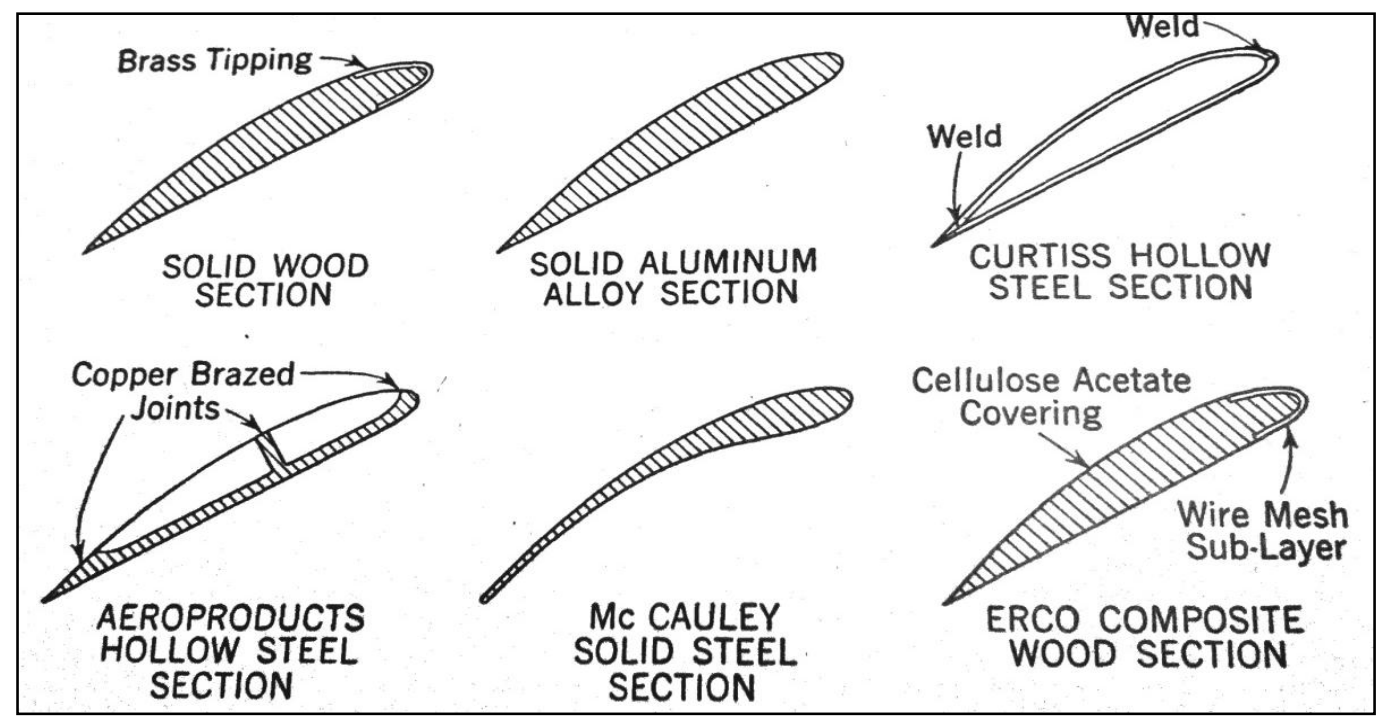

Figure 17. Propeller Manufacturing Techniques (Nelson, 1944) 
A new wave of research into propeller theory surfaced in the second half of the $20^{\text {th }}$ century, as multi-bladed devices for changing rotational power of an aircraft engine into thrust power became a more recognized method of propulsion. An air propeller operates in a relatively thin medium compared to a marine propeller, therefore, is characterized by relatively large diameters and higher rotational speeds (Dommasch, 1953). They are usually mounted directly on the engine drive shaft in front of, or behind, the engine housing and can have two, three, or four blades. In high speed or high powered airplanes, six or more blades can be used and in some cases these propellers have an equal number of opposite rotating blades on the same shaft. These are known as dual-rotation propellers.

A propeller blade advances through the air along an approximate helical path which is the result of its forward and rotational velocity components which impart a force on the surrounding fluid (see Figure 19). This action is similar to a screw being turned into a solid surface, except that in the case of the propeller a slippage (Figure 18) occurs because air is a fluid, and shows the physical relationship between the pitch angle $(\beta)$ and the effective pitch angle $(\varphi)$ (Nelson, 1944).

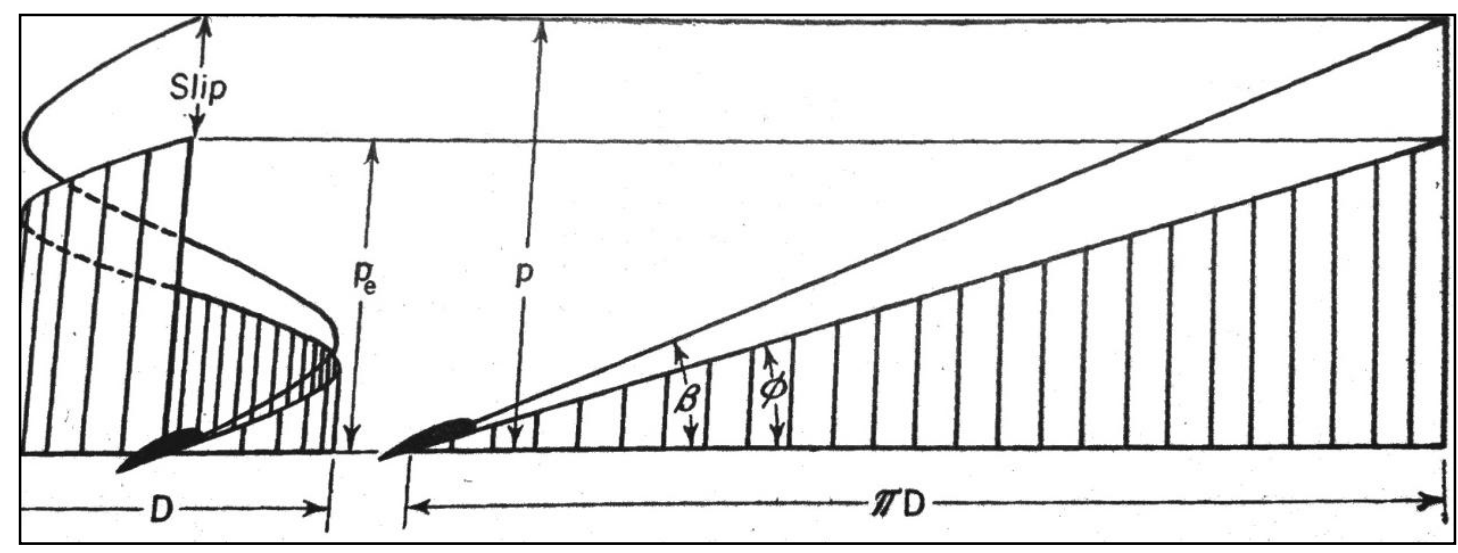

Figure 18. Slipstream of a Generic Propeller (Nelson, 1944)

To rotate the propeller blade, the engine exerts a torque force. This force is reacted by the blade in terms of lift and drag force components produced by the blade sections in the opposite direction. As a result of the rotational forces reacting on the air, a rotational velocity remains in 
the propeller wake with the same rotational direction as the propeller. This rotational velocity times the mass of the air is proportional to the power input. The sum of all the lift and drag components of the blade sections in the direction of flight are equal to the thrust produced. These forces react on the air, giving an axial velocity component opposite to the direction of flight (Figure 19). By the momentum theory, this velocity times the mass of the air going through the propeller is equal to the thrust (Stepniewski, 1984).

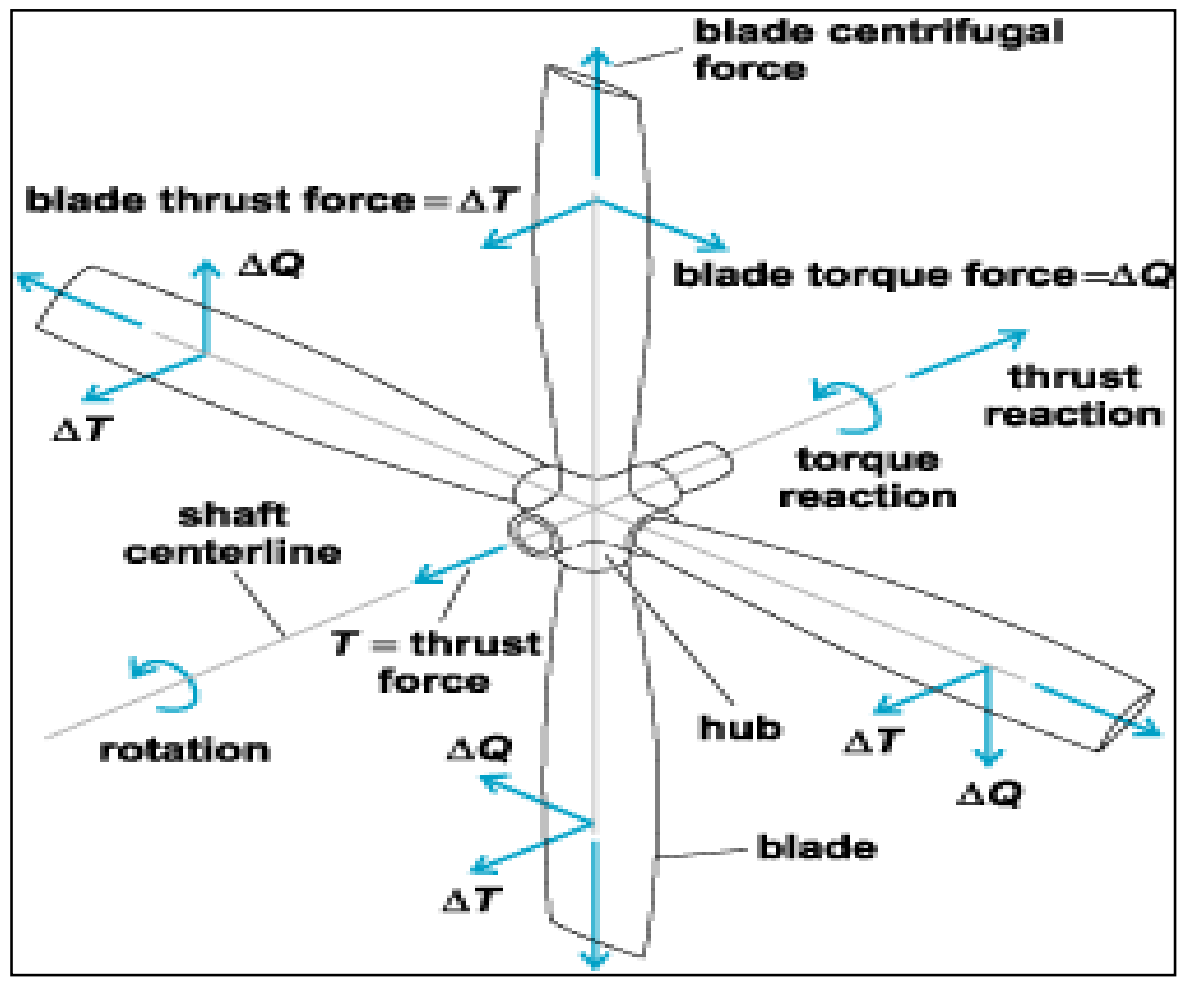

Figure 19. Force Breakdown on a Conventional Four-Bladed Propeller (Stepniewski, 1984)

A propeller blade must be designed to withstand very high centrifugal forces. The blade also must withstand the thrust force produced plus any vibratory forces generated, such as those due to uneven flow fields or the force transferred from the power plant. To withstand the high stresses due to rotation, propeller blades have been made from a number of materials, including wood, aluminum, hollow steel, or plastic composites. The most common material used in the past has been solid aluminum because of its ease of machining and high strength-to-weight ratio. 
However, in today's propeller research and development, composite blade constructions are the new norm for turboprop applications. It has been found that given the correct mixture of resin and layered materials, composite material propellers are more light-weight and have higher strength characteristics than the traditional aluminum blades. Also, since it requires very little post processing once the pattern is made, the cost to make composite blades is reduced in comparison to spending hours having a robot machine a propeller blade out of a solid piece of aluminum (Bedell, 2008).

Some of the historical formulae that are helpful when experimentation with propellers in both a wind tunnel environment and a computational environment are numerous and depend on what outputs the research trials are based around. For this study the main formulae will center on the study of thrust $(T)$, torque $(Q)$, and efficiency $(\eta)$ (Nelson, 1944, Lowery, 1999, Diehl, 1934). The equations generalized for this study are given below as Equation 15 through Equation 20. Comparison of these values between both the experimental propeller and the theoretical (original un-augmented) propeller will give insight into the effect circulation control has on the propeller dynamics. Specifically, the interest of this study is the effect on thrust force produced and torque required to turn the propeller, leading to an overall efficiency change between the unaugmented and the $C C$ activated propeller models.

$$
\begin{array}{cc}
T=C_{T} \rho n^{2} D^{4} & \text { Equation } 15 \\
Q=C_{Q} \rho n^{2} D^{5} & \text { Equation 16 } \\
P_{r}=2 \pi n Q=V^{*}(I)=I^{2} R_{*} & \text { Equation } 17 \\
P_{r}=C_{P *} \rho n^{3} D^{5} & \text { Equation 18 } \\
\eta=\frac{\text { Output }}{\text { Input }}=J \frac{C_{T}}{C_{P *}}=\frac{T V}{2 \pi n Q} & \text { Equation 19 } \\
J=\frac{V}{n D} & \text { Equation 20 }
\end{array}
$$


When examining propeller aerodynamics, it can be easily paralleled to an airfoil in a flow field. The thrust force of the propeller is synonymous to the lifting force, and the input torque is analogous to the drag force. From the motor perspective, the force required to turn the propeller is the torque, i.e. the aerodynamic drag. Efficiency of the propeller, like most similar calculations is merely the ratio of the output power to the input power. This can be a useful calculation if the efficiency rating is based on the forward advance ratio $(J)$ providing a general relation to the range of the aircraft based on power input. The application and study of the aerodynamic forces across a range of advance ratios also allows for comparison of performance over many different blade planforms and propeller geometries due to the unit-less nature of the coefficients of thrust, torque, power, advance ratio and efficiency.

\section{Experimental Testing Facilities}

Since propeller design is a highly publicized scientific art, several previously employed testing methods were studied for potential use in experimental trials. Figure 20 shows the National Aerospace Laboratory (NAL) test set-up found in the Netherlands where scientists used a pressure rake directly behind the propeller plane to measure pressure forces experienced downstream of the rotating plane. Using equations relating to disk actuator theory, as defined in previous sections (Nelson, 1944), it is possible to extrapolate the thrust produced by the propeller being tested according to the pressure change across the blade plane. Another important note about the NAL experimental set-up is that the attachment device is not rigidly connected to the wind tunnel, in order to reduce vibration readings. Also, the mounting sting is positioned downstream of the rotating body in order to minimize the effect it has on the upstream air the propeller interacts with. Both of these particular design aspects were incorporated into this experiment, to reduce the amount of vibrations that the model experienced and to remove the motor and mounting sting from the airflow entering the propeller plane. 


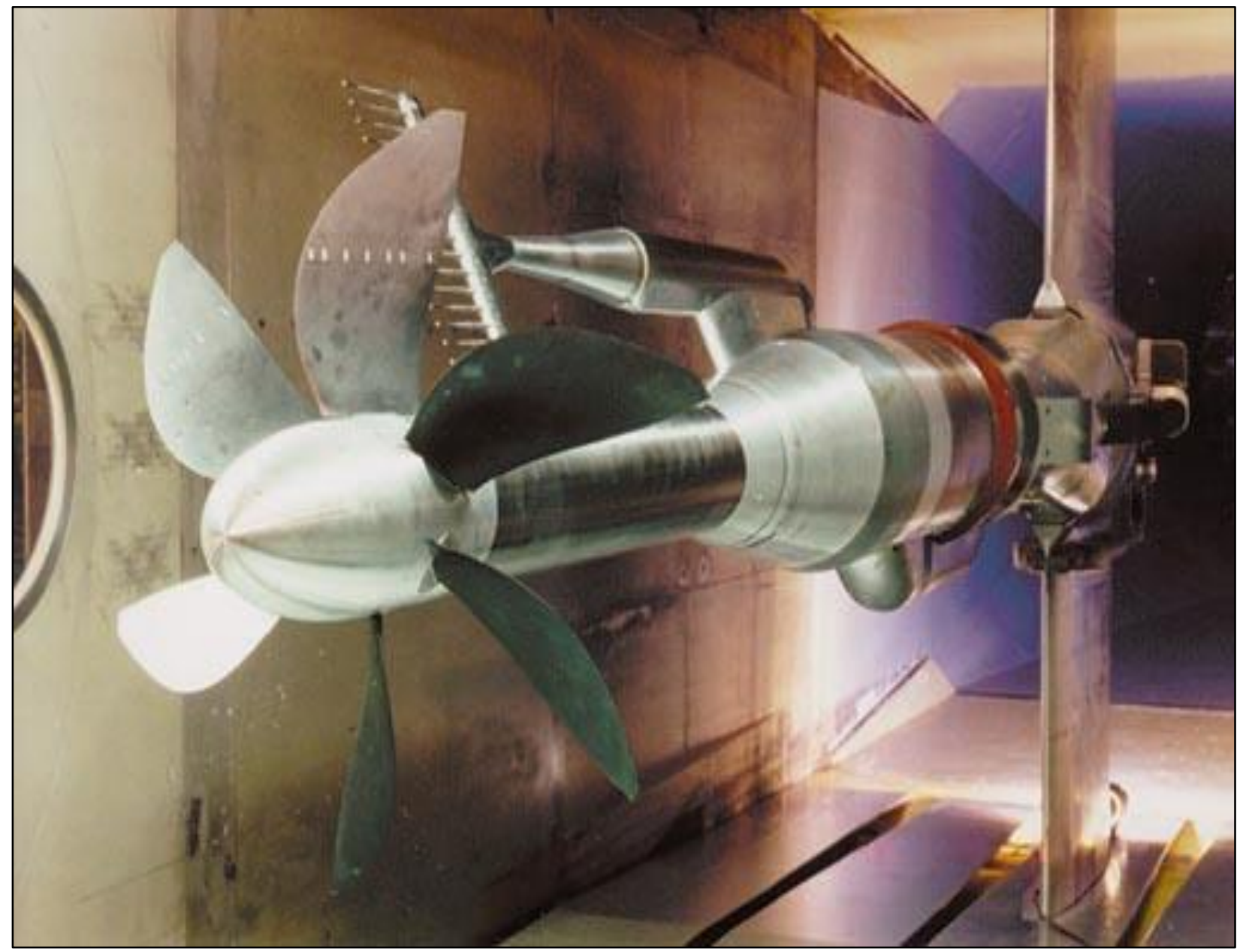

Figure 20. Wind Tunnel Propeller Test Stand (National Aerospace Laboratory)

In Figure 21 below, engineering students from the University of Victoria published work regarding propeller dynamometer experiments, where, like the test stand in The Netherlands, a propeller was mounted upstream of the nacelle with rigid connections in order to reduce streamline interference. Here the apparatus used to move the model in the flow and the sensor equipment is housed outside of the test section. The other major difference between this system and the previous $N A L$ system is the fluid medium. The $N A L$ experimental components focused primarily on airscrew performance testing, while the students at the University of Victoria used their experimental setup in a water tunnel to test aquatic propellers. 


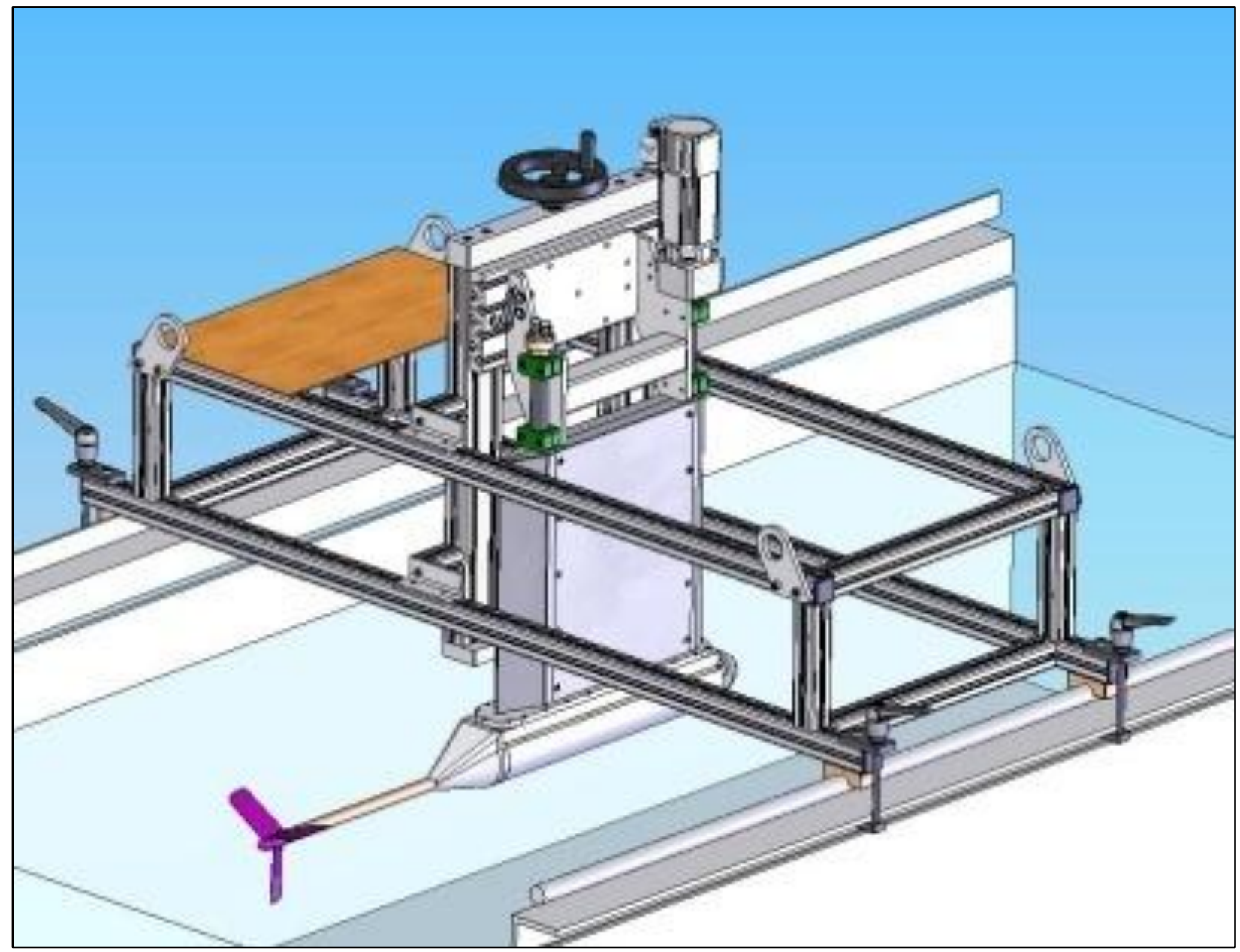

Figure 21. Water Tunnel Propeller Test Stand (Aria Wind Power Systems Inc.)

These are just two of the many propeller test facilities employed in the world. What should be taken from this is that the test fluid is independent of compressibility effects and does not necessarily hold a bearing on the final test results, especially since the final results can be explained in non-dimensional terms and performance curves can then be produced accordingly. As the circulation controlled propeller is predicted to be effective in any fluid medium, and testing commenced as the propeller applied to $U A V$ 's and $A U V$ 's, both air and water can be an effective experimental medium for this test scenario depending on availability.

Another experimental facility is the Vertical Tunnel at the Air Force Research Laboratory, located on Wright-Patterson Air Force Base in Dayton, OH (WPAFB-AFRL). This 
tunnel, houses a vertically oriented cylindrical wind tunnel test section with a diameter of 3.6 meters (12 feet). The air then circulates through the building along the outside walls of the test section funneling back up through the floor and returns through the test area. The outside view of the tunnel is shown in Figure 22.

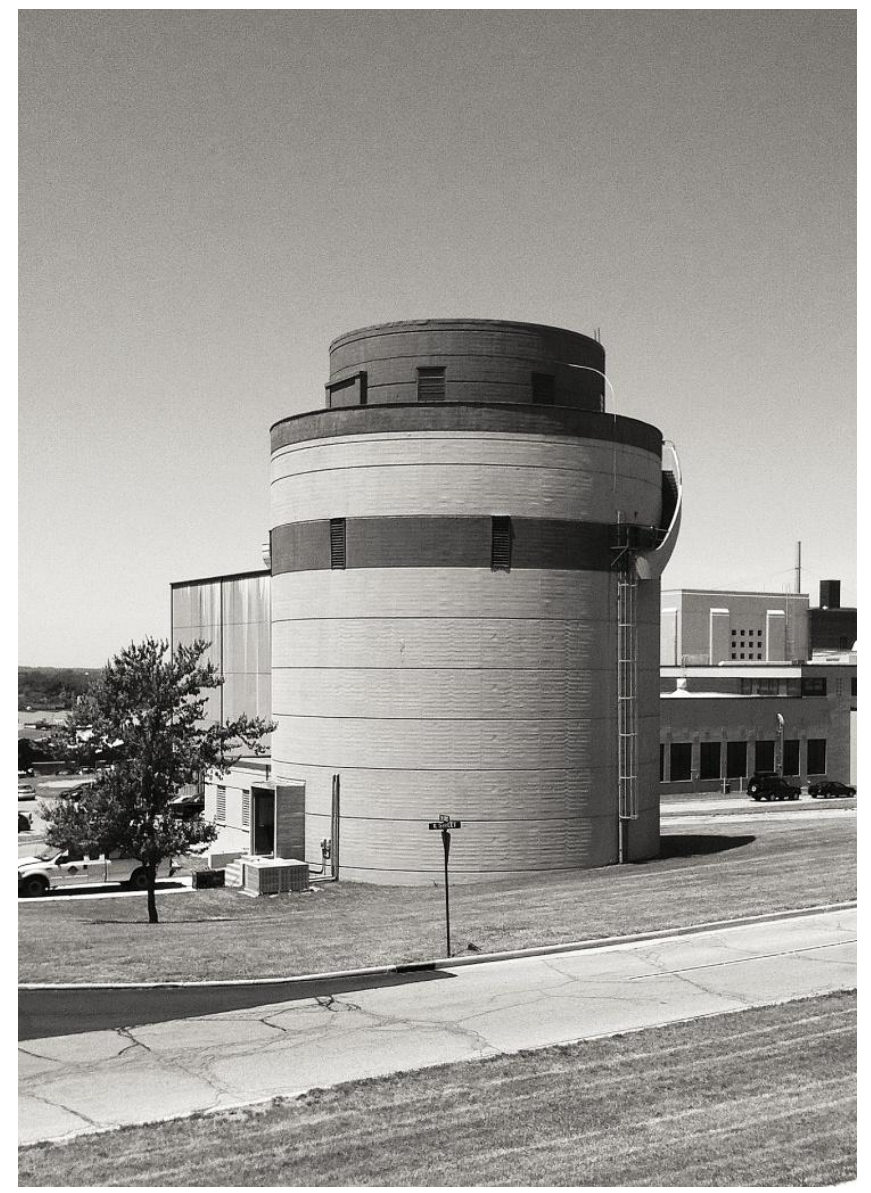

Figure 22. WPAFB-AFRL Vertical Wind Tunnel

The maximum forward speed achievable for testing in this tunnel was 46.1 meters per second (103.0 miles per hour), and has been used previous for rotational models as well as entire fixed-wing aircraft model studies. The test stand in this tunnel is equipped with an 89 Newton (20 pound) force balance with output directly to a data collection interface. It has the ability to be translated in an arc (-30 to 30 degrees with respect to free-stream) mechanically, changing the angle-of-attack of the model with respect to the free-stream velocity allowing for more accurate 
simulations of aerodynamic models. The mechanical nature of the system can also be controlled by the data acquisition system and can be set to log at specific points throughout the translation for specific amounts of time allowing for easy collection of dynamic response data. The test rig can also be manually angled in the off-axis angle between -90 and 90 degrees with respect to the free-stream velocity. The application of this research facility to propeller testing makes experimentation and data collection extremely simple. With the exception of taking into account the wall interference for large scale models, the use of this tunnel can be very helpful in gathering the experimental data needed for a propeller design effort. 


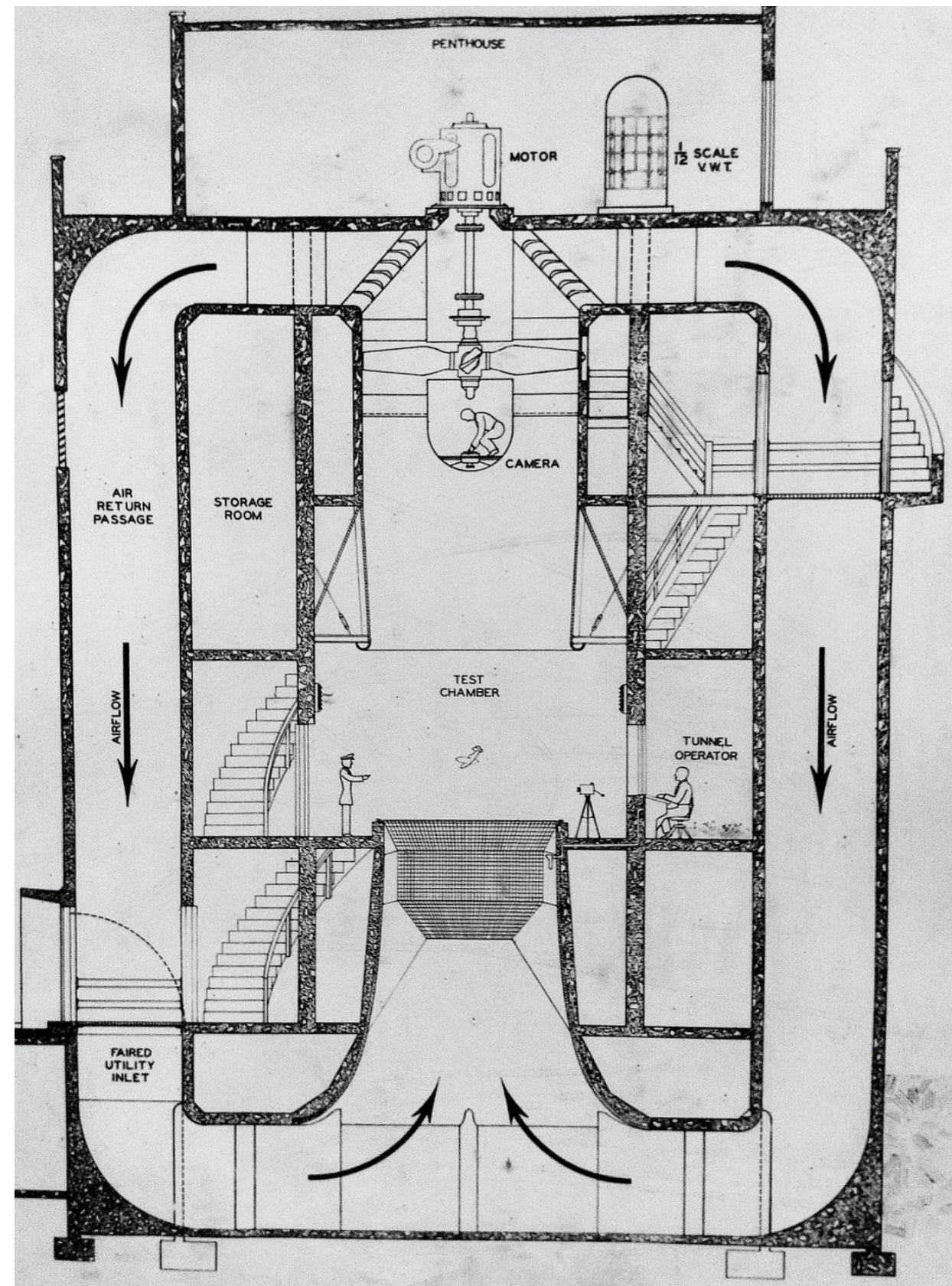

Figure 23. Cross Sectional View of WPAFB Vertical Wind Tunnel

The research laboratories owned by NASA have several tunnels which have been in service for decades used for experimentation on low speed wind tunnel models. These tunnels are scattered throughout the country and all have different capabilities and severely depend on what the input parameters of the model are, specifically the cross-sectional area. Aside from several small-scale subsonic wind tunnels with test section dimensions measured in inches and 
many transonic and supersonic tunnels located throughout the country, there are two specific wind tunnels with large model capabilities available for experimentation, one in Langley, Virginia (NASA-Langley Research Laboratories) and the other in Mountain View, California (NASA-Ames Research Laboratory). Both of these tunnels have historically been used to experiment on models which thoroughly evaluated low-speed characteristics, high-lift application, turboprop performance characteristics, and wake/airflow surveys. Reliability and accuracy of the results studied in these two tunnels are considered among the best in the world.

The NASA-Langley facility houses a subsonic wind tunnel with maximum test section dimensions of 3 meters ( 14 feet) by 6 meters ( 22 feet). This tunnel has the ability to test at forward velocities up to 106 meters per second (237 miles per hour), and Reynolds Numbers values up to and including 2,200,000. This facility is driven by a 12 meter (40 foot) diameter fan with nine blades. Recently this tunnel has been heavily used for $V / S T O L$ research and provides a greater understanding of the theoretical and experimental background involved in high-lift aerodynamics. The availability of this tunnel is the major obstacle in using it for experimentation, while the cost of building the models and renting the space could also become troublesome. The schedule for experimental studies is backed up for years. It is not feasible to wait the extra time to experiment in this facility for a proof-of-concept experiment. Generally this type of experimentation is left for later stages of prototypes.

The NASA-Ames location boasts the largest available test section in the world, with the ability to open one of two test sections one with maximum dimensions of 36 meters by 24 meters (120 feet by 80 feet), and the other with a maximum test section size of 12 meters by 24 meters (40 feet by 80 feet) in the secondary test section. The tunnel uses six fifteen bladed fans, each 12 meters (40 feet) in diameter to pull atmospheric air through the test section. The enormous size of the test sections in this wind tunnel allow for full-scale testing of models up to 30 meters (100 
feet wide), 426 meters (1400 feet) long, and 36 meters (80 feet) high. This allows for testing to be done on real prototypes and avoids the need to use Reynolds Number or Prandtl Number matching schemes to analyze data on small-scale models. The use of this particular wind tunnel is attractive for this experiment because it allows for larger propeller model geometry as well as larger plenum geometry, thus making the manufacturing process much easier. Downsides to experimenting at this facility basically lie in the overall cost of use, specifically, the cost of renting the tunnel, the instrumentation and model design and manufacturing costs of large-scale models as well as the cost of traveling to California for testing scenarios.

\section{Summary of Historical Review}

With the introduction of blown flaps and circulation control in the 1960's the overall articulation of the airfoil has changed at a steady pace from mechanically driven actuators, to using the ability to change the aerodynamics using forcibly blown air located at strategically located ejection slots. Coupled with the Coandă Effect, and understanding of fluid dynamics and mechanics, this principle is the basis for the following research and has the possibility to enhance some of the basic principles of aerodynamics. Using the research background into basic principles of aerodynamics, coupled with propellers, experimental testing techniques, and computational methods, an experimental design has been created, and conducted in order to study the effects of this passively pressurized, circulation control augmented propeller. 


\section{Preliminary Investigation}

Due to the constant environmental and technological need for improvements in propeller performance, it is envisioned that the use of a novel circulation control system can increase the endurance experienced by an aircraft via augmentation of flight efficiency throughout the flight envelope. In this study the application of a passive circulation control system for an aircraft propeller was evaluated. The use of a passive system for circulation control, and the corresponding system to provide air to the blowing slot, are the distinguishing features of this research. The use of the term passive system indicates that mechanical and/or electrical power is not used to supply the airflow directly to the circulation control sub-system. The use of this system can be applied to any rotating object that generates a fluid dynamic force in any fluid medium. However, for simplicity it will be described based on the first envisioned application: a propeller operating in air for an $U A V$.

In traditional flow control applications blowing/suction slots are located on the surface, on the leading and/or trailing edge of an airfoil, that are coupled to a device to create a pressure differential with the surrounding air. In order for an aircraft to gain the amount of lift needed at take-off, and potentially landing, an increase in the thrust potential of the propeller can decrease the runway length required with a relatively small amount of circulation control enhancement. It is currently believed that the rotational nature of a propeller can provide limited pumping capability to supply the circulation control sub-system, mitigating the need for the additional weight of an external pumping system and the associated ducting.

In conventional propeller theory, the free-stream air that is "pulled" into the propeller imparts a pressure on the hub of the propeller. This pressure on the front side of the propeller is greater than the local atmospheric pressure. In fluid dynamics, a fluid flows from high pressure to low pressure, thus for air, or similar fluid, a local velocity can be generated. By strategically 
placing a channel within the hub and through the blades of the propeller the higher pressure at the center of the hub was utilized to drive a flow to the circulation control blowing slot. In addition, the fluid was accelerated due to the centripetal forces applied by the rotational nature of the propeller.

The high pressure at the hub is loosely dependent on the propeller performance, from the standpoint that a higher static thrust propeller will impart more velocity to the surrounding fluid. Thus, any airfoil shape and any propeller diameter can be utilized with the passive circulation control propeller system. The mass flow rate supplied to the circulation control sub-system will vary. The variation in mass flow may require slightly different sized blowing slots and ducting configurations. However, the passive circulation control air, or fluid supply concept remains unchanged. For simplicity in the described experimentation, the model has a nearly symmetric profile, as shown in Figure 24, and uniform taper, Figure 25. For the remaining preliminary analysis a propeller diameter of 4 feet (1.22 meters) has been chosen as the initial apparatus, based on availability of historical data. By implementing an inlet to a fluid flow channel near the hub of the propeller, the elevated pressure supplies the circulation control sub-system with air.

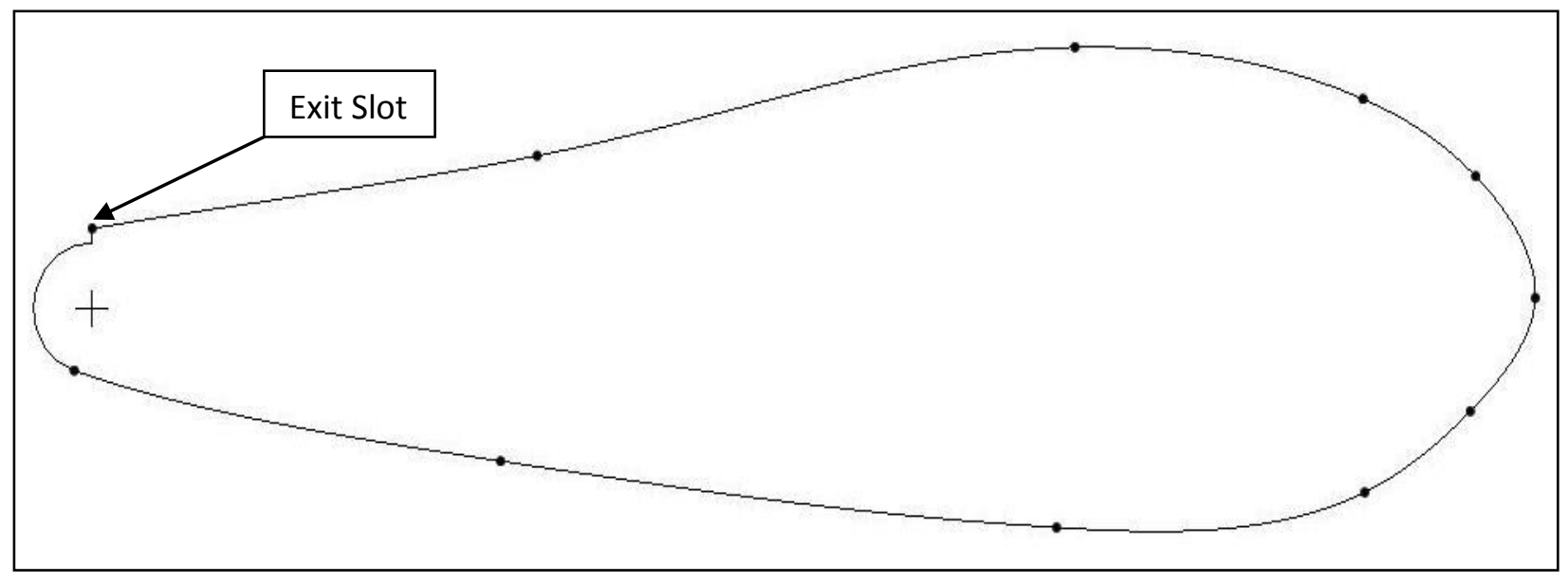

Figure 24. Side View of General CC Propeller Blade (Not to scale) 


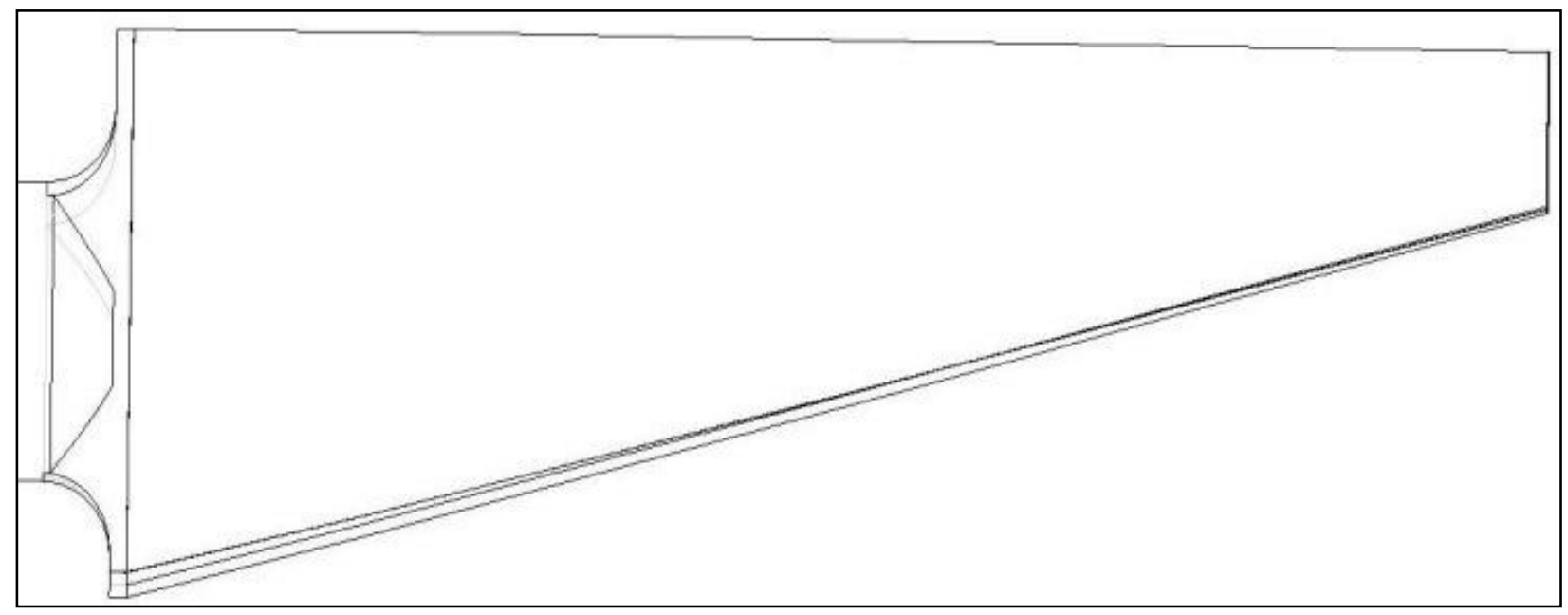

Figure 25. Top View of General CC Propeller Blade (Not to scale)

The passive nature of the system also has an advantage of being constantly enabled, or with a simple valve or gate the use can be restricted only to times where high-lift is desired.

\section{Methodology}

Preliminary impetus for a passive circulation control system installed on a propeller was to address a need for increased flight distance/range of unmanned aerial vehicles, as well as the possibility for applications to autonomous underwater vehicles. Background literature research into the application of a passive circulation control system revealed the amount of potential variables that will have an impact on the design. Among these variables are, slot exit dimensions (length, height, placement along the propeller radius), internal plenum dimensions (choke point, length, volume), capture area for mass flow (to provide necessary mass flow), airfoil shape (radius of rounded trailing edge), effective rotational speed (centrifugal pressurization), altitude effects (density and pressure changes), and finally, the effect of forward velocity on pressurization. The study of these variables shows a general effect that each has on the entire system as well as how they affect one another as a component in the overall circulation control system. 
An acceptable way of studying these factors as components in the design as well as how they affect one another is to conduct a sensitivity analysis. The analysis includes all of the variables which are predicted to have an influence in order to find a predicted trend from previous known historical data. Although this type of analysis highlights the variables which have the greatest degree of impact on the system, and thus eliminates the variables which have little impact, this form of study cannot take the place of conventional testing of a new system. It can be powerful using theoretical data and predictions to obtain an idea of what may happen when a prototype is built and examined. This method is also helpful in predicting the necessary hardware needed to complete testing and analysis of a prototype, including data acquisition components, testing schedule, and experimental setup.

\section{Research Application}

The feasibility of this device is closely related to several areas of interest including the pressurization availability, the increase of induced drag forces due to the addition of a rounded trailing edge, and finally the addition of a mass capture device on the front plane of a propeller.

The rounded trailing edge of circulation control devices has been shown to increase the drag in comparison to that of non-circulation control bodies of the same basic shape, with a sharp trailing edge. In general circulation control cases, the increase in lift greatly overcomes the increase in drag on the aerodynamic body and the increased thrust needed to drive the circulation control system is not significant in comparison to the advantages of the greater capacity for lift. It is also a well-studied fact that the drag increase on the rounded trailing edge or the circulation control body is greatest when the circulation control system is first turned on or cycled between on and off positions. The proposed system, again, is a passive system and is never turned off. With this idea in mind, it is predicted that without needing to turn the system on and off it will perform in a better capacity to increase lift forces of the propeller, and/or reduce the amount of 
input power needed to turn the propeller, thus increasing overall efficiency and aircraft flight range.

Although there are several references in the literature for the use of twice the free-stream velocity as a standard minimum, as the exit velocity for circulation control slots, it has also been shown in more recent studies that is it not necessary to have such an exit velocity to have an impact on the circulation of the streamlines around an aerodynamic body (Englar, 1987, Loth 1984, Franke, 1987). Assuming the internal plenum of the circulation control airfoil can only be pressurized to slightly above atmospheric pressure, the exit jet of the circulation control slot will still be able to produce a velocity greater than the original free-stream velocity of the airfoil. This increases the value of the velocity ratio $\left(V_{\infty} / V_{j}\right)$ to above 1.0 .

Because this proposed system is only attempting to produce a single digit percentage thrust/range improvement it does not need to improve the overall lift by 3 to 4 times as can be achieved with most active circulation control systems. The traditional circulation control types of systems previously studied provided an exit jet velocity twice that of the free-stream systems and looked to multiply the lifting performance of the aerodynamic body by more than single digit percentages, as is desired in this particular application.

The addition of a mass capture device on the frontal stagnation area of the propeller hub at this point of the research is merely a concept and needs to be studied further. In the preferred design, the capture device would be situated inside the existing hub space and not add an additional frontal area to the propeller hub. The addition of drag forces due to this apparatus will be less than even the current cone design because of the suction forces predicted to siphon mass and pressure into the internal plenum of the circulation control system which is predicted to reduce the overall stagnation force on the hub. It is the pressure force from this stagnation area 
added to the centrifugal forces from the rotating propeller that provides the motive force for implementation of this passive system.

According to previous studies and Equation 21, a jet momentum coefficient $\left(C_{\mu}\right)$ of 0.1 can be used to provide a lift coefficient increase of 3.0 at a plenum pressure to free-stream pressure ratio of $1.25\left(P / P_{\infty}\right)$, similar to the maximum pressure calculated due to stagnation forces (Englar, 2004).

$$
C_{\mu}=2 h_{j} V_{j}^{2} /\left(c V_{\infty}^{2}\right)
$$

Equation 21

\section{Sensitivity Analysis}

In order to begin the process of designing and conducting an experiment, it is necessary to look at all of the variables that are considered to have a potential impact on the final result of the propeller performance. In the case of a passively pressurized, circulation controlled propeller, as in this research, there are several factors which needed to be studied. Among these important variables are parameters such as free-stream velocity, rotational speed of the propeller, propeller diameter, sweep, area and volume of the circulation control ducts and plenums, slow exit area, exit pressure/velocity, mass flow rate, and fluid density. Also needed to be examined are certain blade characteristics such as twist, chord length, and cross-section. The final area of concern in this research was the design of a capture device which will need to efficiently capture free-stream air to supply pressurization to the internal circulation control plenum of the propeller. The capture device areas of interest included inlet and outlet areas, mechanical design, and rate of fluid rejection. This section explains some of the preliminary work conducted in order to determine the impact these variables will have on the entire system, in preparation for computational and experimental studies.

Before the results of this analysis are discussed, it should be noted that all of the preliminary investigations of the variables were studied as they related to their use in conjunction 
with $U A V / A U V$ design ranges. Simply, the preliminary idea of use for this technology is to apply it to unmanned aerial vehicles and possibly to autonomous underwater vehicles. This is just regarded as a starting point for the research, and it is hypothesized that this technology will be advantageous in any fluid medium and in various arrays of implementation.

\section{Effect of Forward Velocity on Inlet and Outlet Pressures}

As explained earlier in the fluid mechanics background, the inward velocity through the rotor plane can be converted to a pressure force on the propeller hub, and through an analysis of the Bernoulli Equation the relationship between pressure and velocity is defined by Equation 22 (Dommasch, 1953). With an inlet on the propeller hub feeding to a channel in the propeller

blade, pressurized air induced from forward velocity $\left(V_{\infty}\right)$ can move through the inlet at the high pressure stagnation location, on the front of the propeller, to a low pressure region (atmospheric, or less) at the channel exit.

The Bernoulli Equation can be used to estimate the inlet pressure based on the assumption that there is a stagnation point at the hub and the initial flow conditions at an elevation $(z)$ are seen as atmospheric pressure $(P)$, density $(\rho)$, and specific heat ratio $(\gamma)$ and the forward speed $(V)$ of the inflow of air is zero. Conversely, when the propeller operates in a compressible fluid, similar to air above a speed where the Mach Number reaches 0.30 or more, it is necessary to calculate the effect inside the plenum using Equation 23, where the gravitational constant $(g)$ can be taken as the rotational component of the mass spinning with the propeller and the radial distance $(r)$ is the distance away from the center hub along the propeller. Using this equation, the pressure can be predicted along the length of the blade at several different radial locations incorporating the effect of centripetal forces on the mass of air.

$$
P+\frac{1}{2} \rho V^{2}+\gamma z=\text { constant }
$$

Equation 22 
As an estimate of the forward flight speed impact on the pressure changes on the hub of the propeller, the total pressure as determined from the Bernoulli Equation was used to generate Figure 26 which indicates that at a forward flight speed of $120 \mathrm{~m} / \mathrm{s}(393.7 \mathrm{ft} / \mathrm{s})$ a pressure of 1.10 atm is predicted due to stagnation forces directly upstream of the propeller plane. This trend is shown for a rotational speed of $1,000 \mathrm{rpm}$ and predicted for a blade diameter of 0.40 meters (1.3 feet). This increase in pressure due to the flight speed allows for a greater flow rate of the captured air for circulation control uses.

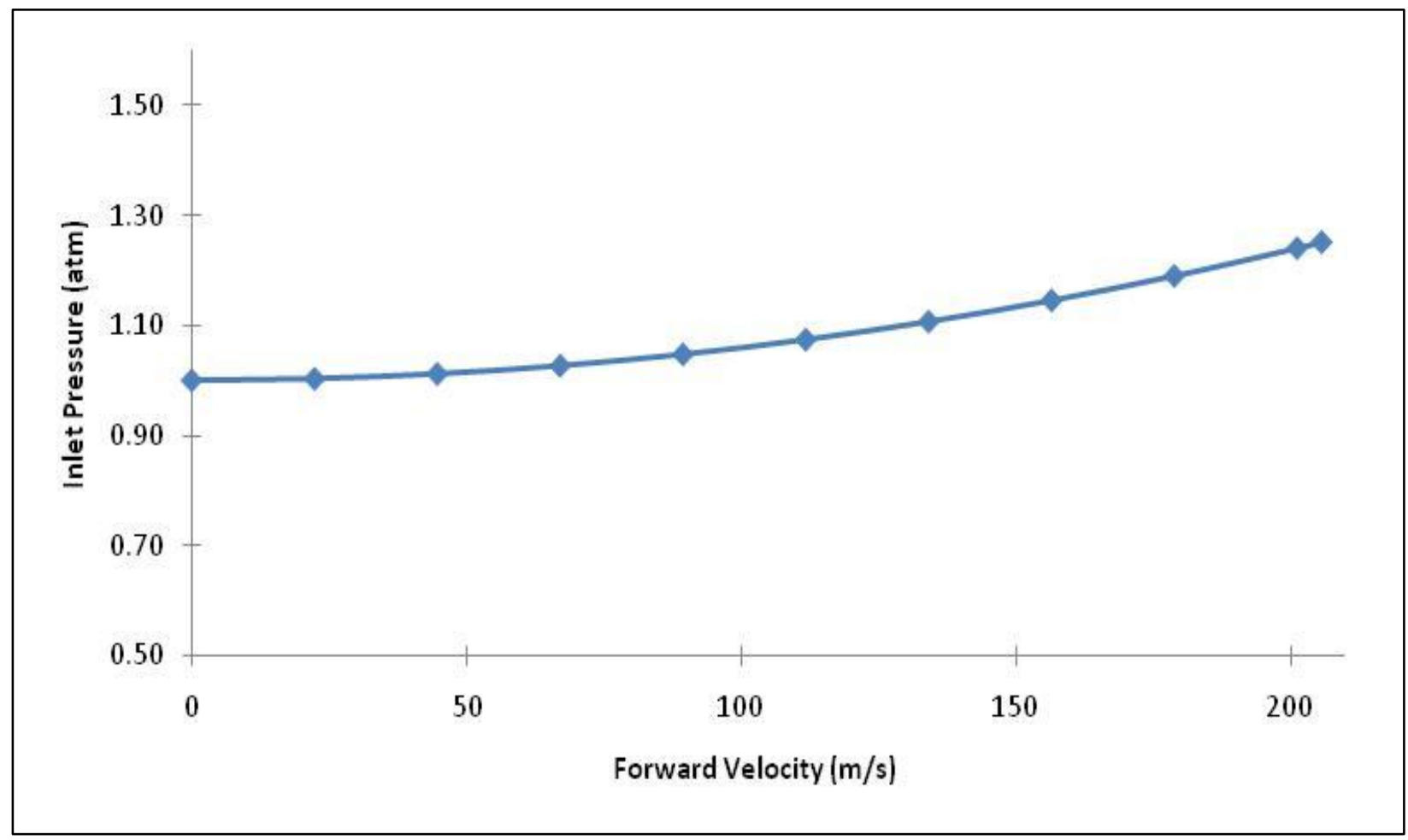

Figure 26. Trend Study of Forward Velocity Effect on Circulation Control Inlet Pressure (Kweder, 2010)

In Figure 27, several propeller rotational speeds are shown having similar trends. The trend studied at 1,000 rpm is shown again as in the previous plot, however, two other rotational speeds are given as well, 8,000 and 16,000 rpm. This also shows a similar predicted trend of 
pressure addition to the atmospheric value due to forward flight speed. At a similar forward velocity of $120 \mathrm{~m} / \mathrm{s}(394 \mathrm{ft} / \mathrm{s})$ the study at 8,000 rpm predicts an increase of $0.10 \mathrm{~atm}$ where the study also predicts a $0.09 \mathrm{~atm}$ increase at 16,000 rpm. Differences in the predicted pressure increases are caused by suction forces on the propeller at each rotational speed as well as a change in the stagnation force on the propeller. A higher rotational speed produces a greater suction force on the upstream face of the propeller. Each of these three studies was performed using a blade diameter of 0.40 meters ( 1.31 feet $)$. This estimation does not, however, take into account the effect that the centripetal acceleration has on the mass of air in the circulation control slot. The effect centripetal acceleration has on the internal plenum pressure will be discussed in depth further in this project.

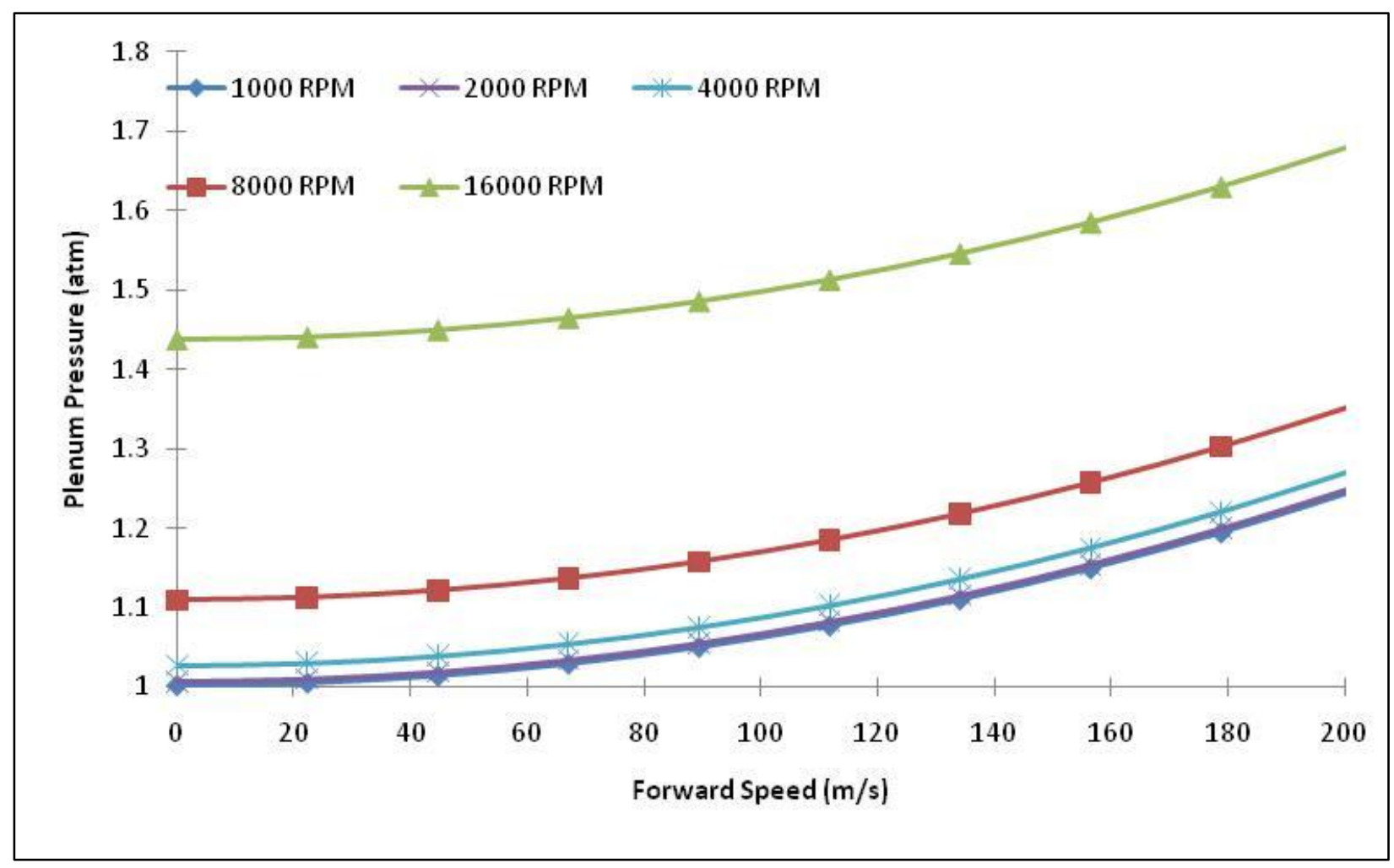

Figure 27. Effect of Forward Speed on Plenum Pressure (Kweder, 2010)

In order to verify the results of the trend study found above, it was necessary to use previous experimental data (Englar, 2005). The results of the study used to verify the predicted 
results are given in Figure 28, below. In this figure, it is shown that a much larger jump in jet exit velocity is predicted at between $2000-8000 \mathrm{rpm}$ in comparison to the difference in output pressure while the propeller operates between 1000-2000 rpm. In the historical study, the results show the slot exit velocity over a range of airspeeds. Assuming similar ducting areas and rotational speeds, Figure 28 shows an approximate fit to the data studied by Englar in 2005. Englar's work, as in this study, shows that as the forward speed increases, the exit velocity of air used in circulation control applications also increases, therefore having a greater effect on the streamlines of the airfoil. Using the results of this 2005 study, it is possible to predict that at higher forward velocities, higher exit velocities are available for circulation control.

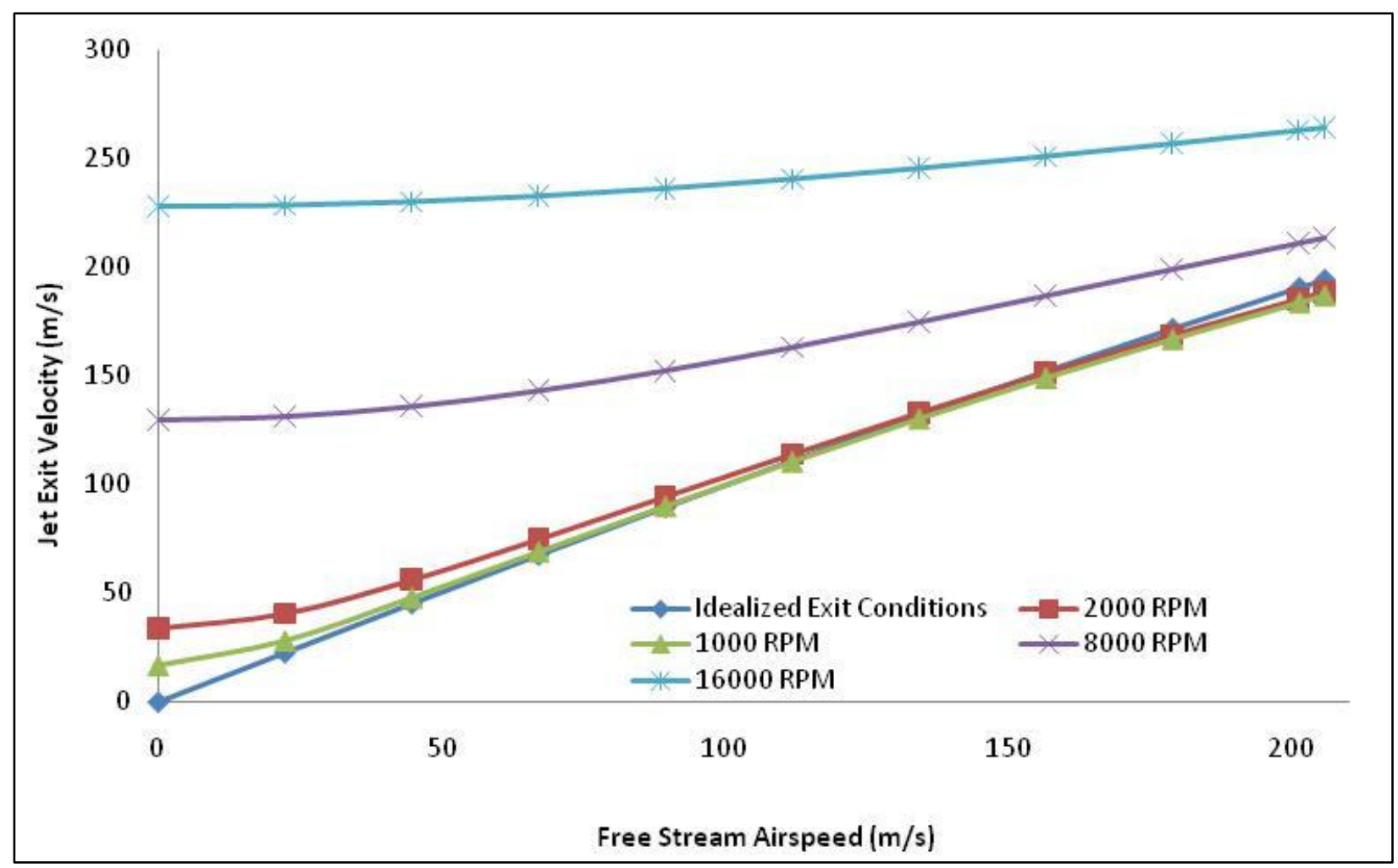

Figure 28. Effect of Forward Speed on Exit Velocity (Kweder, 2010)

\section{Effect of Propeller Diameter on Change in Pressure}

According to propeller theory, the thrust of a specified propeller can be related to a change in pressure between the upstream and downstream pressures of the propeller disc. 
Idealizing the propeller plane as a thin disk in a slipstream field of flow, the thrust $(T)$ produced by the propeller is directly proportional to the frontal area on the propeller $(A)$ and the squares of the upstream $\left(V_{s}\right)$ and downstream $\left(V_{o}\right)$ velocities as shown in Equation 24 (Nelson, 1944).

$$
T=\frac{A \rho}{2}\left(V_{s}^{2}-V_{o}^{2}\right)
$$

In order to relate the thrust to the pressure differential, the Bernoulli Equation, Equation 12, is solved for the pressure difference $(\Delta P)$ in Equation 25, which, when substituted into Equation 24, results in Equation 26 for finding the thrust output $(T)$ of the propeller (Nelson, 1944).

$$
\begin{gathered}
\Delta P=\frac{1}{2}\left(V_{s}^{2}-V_{o}^{2}\right) \\
T=A \Delta P
\end{gathered}
$$

Equation 26

With a value for thrust related to the pressures upstream and downstream of the propeller disk, Figure 29 was created to show the effect that the propeller diameter has on the pressure change across the disc. As the propeller diameter increases, the difference in pressure upstream and downstream of the blade decreases significantly. This suggests the pressure differences that are most useful in passive circulation control applications are found toward the lower end of the propeller diameters. This is due to the fact that this particular circulation control application takes advantage of the pressure difference between the upstream and downstream sides of the propeller in order to pressurize the plenums inside the blades.

In keeping with $U A V$ applications, the diameters of the propellers used are generally between 0.20 and 0.50 meters $(0.66-1.64$ feet). According to this trend, the propellers in this area see around $0.50-0.75$ atm pressure change. This change is predicted to be adequate air pressure to run boundary layer control and/or circulation control applications assuming the exit slot can be designed to provide the required exit slot ejection velocity. The trend shown in Figure 
29 predicts that there is little effect on the change in pressure over the entire blade due to horsepower changes, the effective doubling of the output horsepower only changes the differential pressure a maximum of $0.01 \mathrm{~atm}$ over a 0.10 meter $(0.33$ feet $)$ diameter propeller.

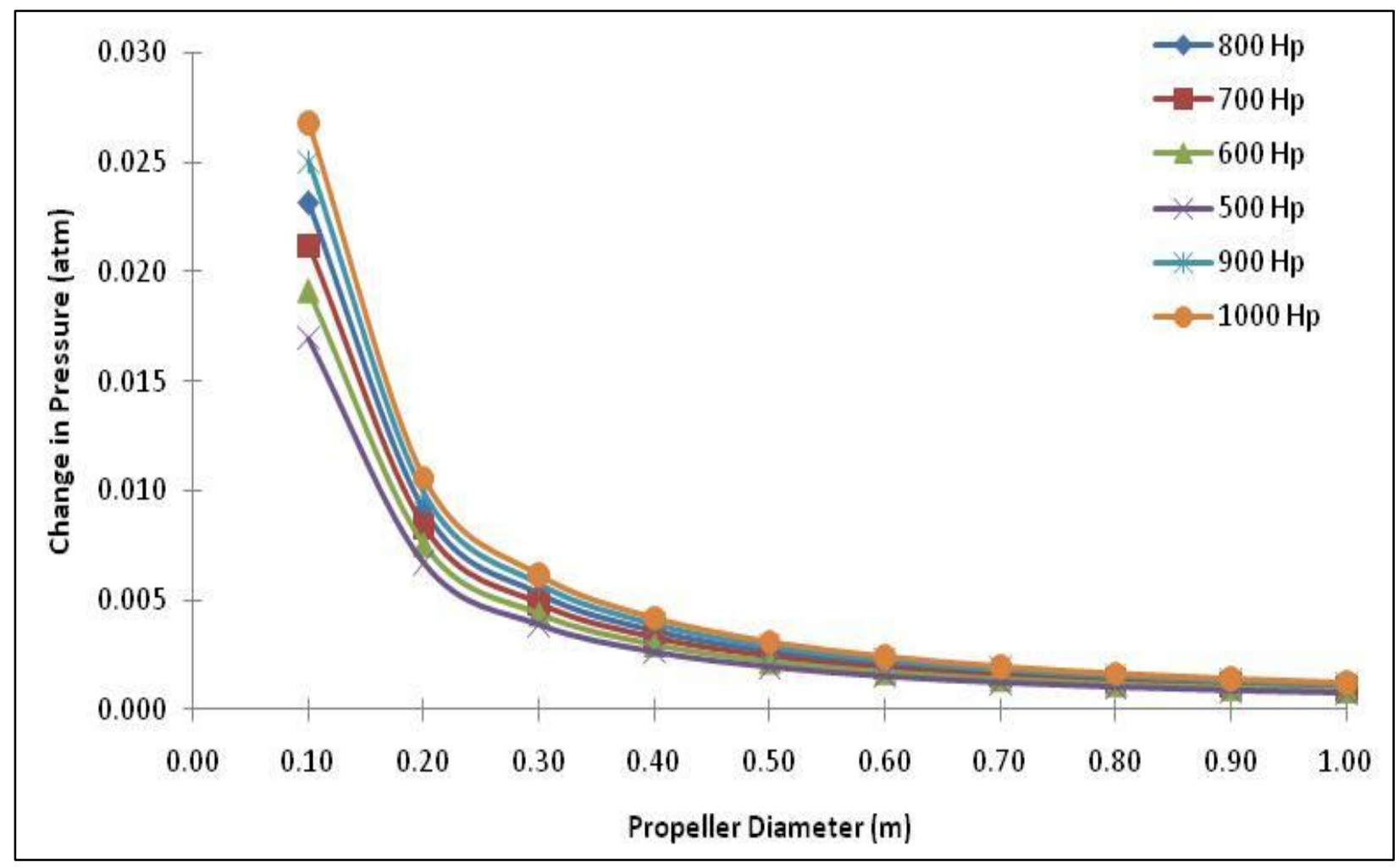

Figure 29. Change in Pressure as it Relates to Varying Propeller Diameter (Kweder, 2010) Effect of Blade Diameter and Centripetal Acceleration on Plenum Pressure

It is currently envisioned that a constant cross-section capillary will be used initially throughout the blades of the propeller, minimizing the regions in which pressure can buildup and restrict the flow. When a full design is undertaken, it will be necessary to attempt to make the exit slot the limiting dimensions of the channel in order to prevent flow deceleration through the capillaries of the propeller blades. This can become a challenging task as there is only so much room available for use on the interior of a propeller blade.

It is important to note that the use of a constant cross-section is not a requirement of the design, it was chosen for simplicity and ease of fabrication. The static pressure outside the 
blowing slot was considered to be standard atmospheric conditions, thus the stagnation pressure is elevated and the blowing slot analyzed as a nozzle with a specified back pressure (inside the flow system) and surroundings. Another form of the Bernoulli Equation was used to determine the exit velocity of the passive circulation control augmented propeller. Equation 27, from (Englar, 2005), which is a derived expression based on the Bernoulli Principle, Ideal Gas Law, and Isentropic Fluid Equations, was also used to verify the magnitude of the jet exit velocity. This equation is based on the specific heat ratio $(\gamma)$, and the gas constant $(R)$ of the fluid as well as the temperature (Temp) and internal pressure $(P)$ of the plenum. As noted above, it is necessary to take into account the pressurization of the plenum due to centripetal forces using Equation 23 when the propeller operates above sonic speeds and a normally incompressible fluid, such as air, becomes a compressible fluid.

$$
V=\sqrt{\frac{2 \gamma R(\text { Temp })}{1-\gamma}\left[1-\left(\frac{P_{\infty}}{P}\right)^{\frac{\gamma-1}{\gamma}}\right]}
$$

Below in, Figure 30, a trend study of plenum pressurization capabilities due to centripetal acceleration as well as forward velocity was performed on varying blade sizes ranging from 0.10 meters to 1.00 meter $(0.33-3.30$ feet $)$ in diameter. Also, for simplicity, the channel area is assumed to be square at 0.05 by 0.05 meters ( 0.164 by 0.164 feet). The trend shows a linear-like increase in the amount of pressure in the plenum as the rotational speed increases as well as when the propeller diameter increases. It should be noted that in historical studies it has been shown that, as the tip speed of the airfoil reaches transonic or supersonic speeds, the tip speed surpasses the local speed of sound and the performance of the propeller is no longer predicted to increase. 


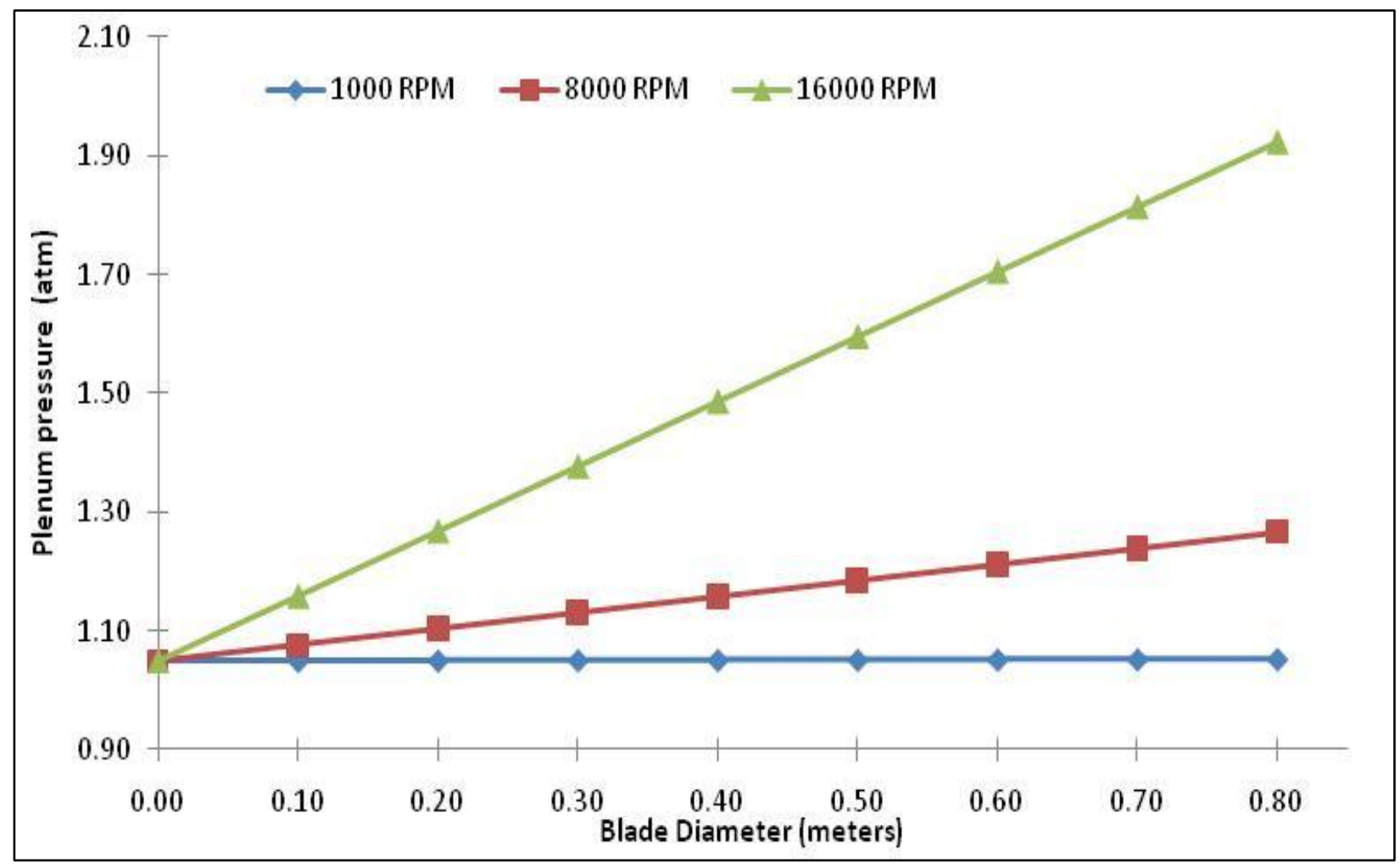

\section{Figure 30. Plenum Pressure versus Blade Diameter Trend Study as a Function of Propeller Rotational Speed (Kweder, 2010)}

Centripetal force on the fluid medium used for circulation control is another area of interest in understanding how the fluid reacts inside the plenum. Figure 30 shows the effect predicted to be experienced by the fluid due to rotation. This preliminary study shows that the centripetal force experienced by the fluid while rotating has a large impact on the internal pressurization of the circulation control plenum of the proposed augmentation. This trend study was completed using predicted data from a 1.2 meter (4.0 foot) diameter propeller spinning at three different rotational speeds and predicts that the majority of the internal pressure useful as a circulation control air supply will be provided by the centrifugal accelerations acting on the volume of air while the blades are turning and not due to the forward velocity of the aircraft. 
Ideally, the data in Figure 28 and Figure 30 would be combined in order to see the full effect on the plenum, however, the final important design effect still needs to be incorporated, that being the duct sizing and exit slot dimensions (described in the next section).

\section{Ducting Dimensional Effects}

As mentioned earlier, the circulation control capillary design inside the rotating body of an aircraft propeller can prove to be a challenging portion of the design effort. The most common mistake found is that the plenum design does not allow for the circulation control exit to be the limiting dimension of the fluid duct. This causes areas inside the pressurized plenum to become the smallest dimension orifice, acting as the area which restricts the flow rate of the fluid through the system.

In order to avoid this problem, most previous circulation control designs have allowed for the entirety of the internal volume of the airfoil to be pressurized, making the circulation control ejection slot the limiting cross-sectional area. As such, for this study, it was necessary to establish the effects that some of the interior dimensions of ducting will have on the internal pressurization of the proposed propeller. In Figure 31 and Figure 32, again, three different rotational speeds of a 0.406 meter (1.3) diameter propeller were studied and the interior slot volume was varied by changing the cross-sectional area while keeping the internal slot length constant (Figure 31), as well as varying the internal slot length while keeping the cross-sectional area constant (Figure 32). This study was conducted in order to view the predicted plenum pressure change that would be experienced inside the proposed circulation control blade. As assumed, as the interior space able to be used for circulation control increases the amount of circulation control pressure that is available due to forward speed and centripetal forces acting on the captured air increases drastically. The highest amount of interior plenum pressure can be affected by increasing the duct length inside the proposed propeller and allowing the centripetal 
forces to act on the fluid to a greater extent as shown in Figure 32; here outlet pressures are predicted to get as high as $1.28 \mathrm{~atm}$.

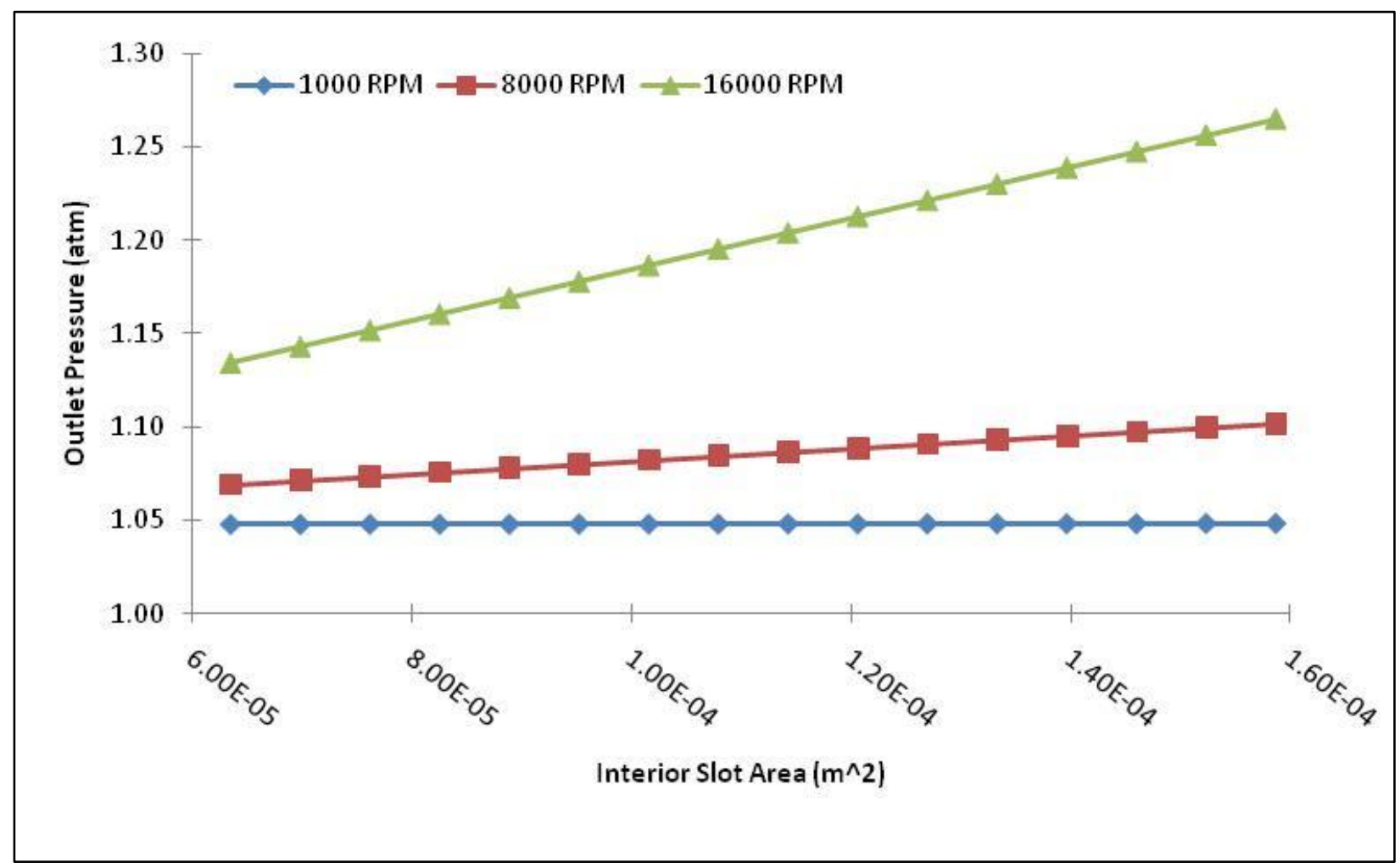

Figure 31. Effect of Change in Plenum Cross-sectional Area on Outlet Pressure (Kweder, 2010) 


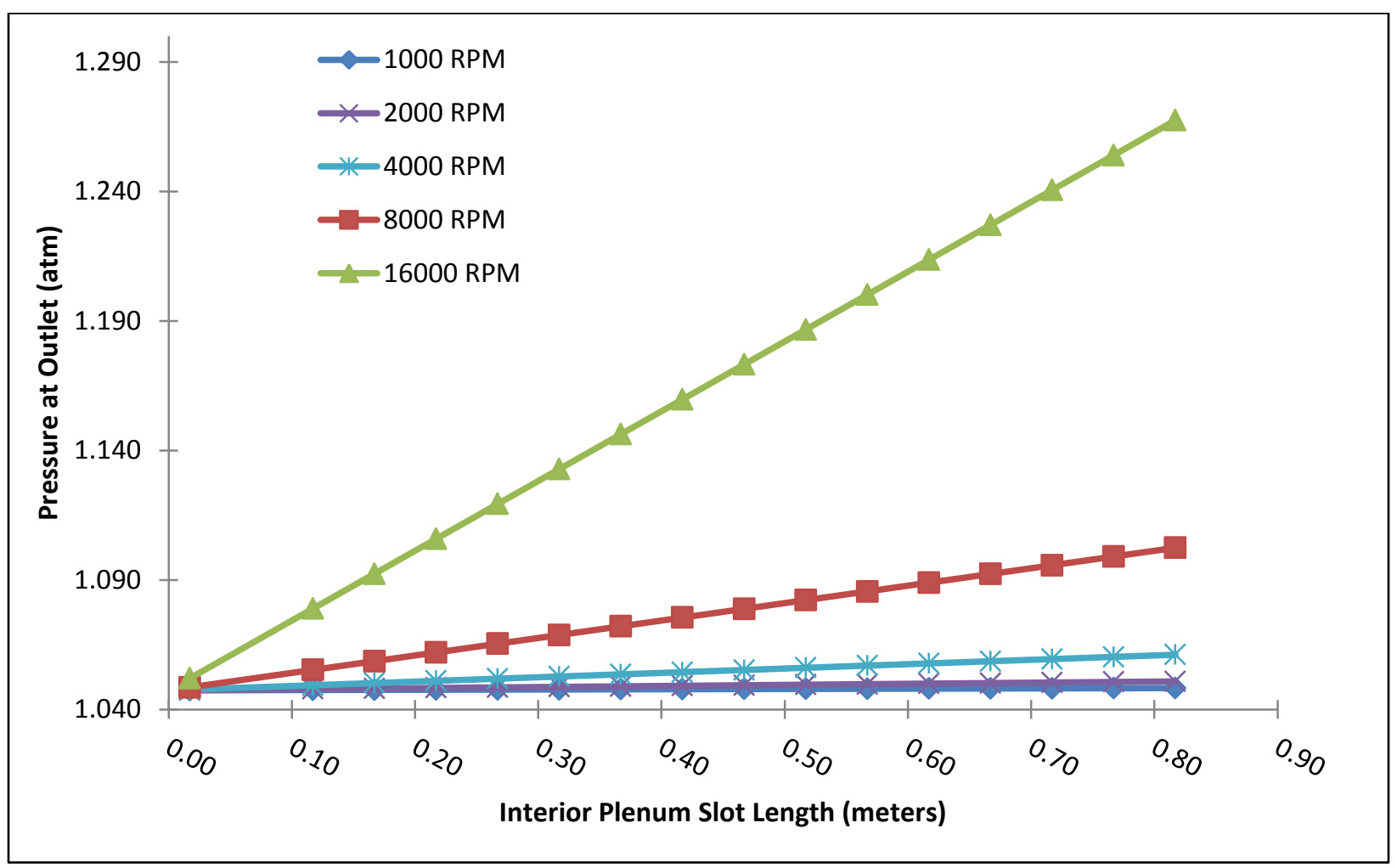

Figure 32. Effect of Interior Duct Length on Outlet Pressure (Kweder, 2010)

Preliminary investigation of the proposed passive pressurization system applied to one of the blades of the NACA 4-(5)(08)-03 ( $C_{l}$ design of 0.500$)$ two bladed propeller, predicts a pressurization design change in lift coefficient averaged over the propeller blade of 0.071 and an overall change at the design $C_{l}$ of 0.090 , as shown in Figure 33.

The predicted lifting force change can be found using an empirical relation, shown in Equation 28 (Loth, 1984).

$$
\Delta C_{l}=40\left(\frac{h}{c}\right)^{0.64}\left(\frac{V_{j}}{V_{\infty}}-1\right)
$$

Equation 28

In this empirically derived equation, the change in lifting coefficient, $\Delta C_{l}$ is a function of the slot height-to-chord-ratio $(h / c)$ of the airfoil and the jet exit velocity-to-free-stream-velocityratio $\left(V_{j} / V_{\infty}\right)$ ratio. This increase is purely in the lifting forces applied to the propeller and with a slot exit velocity between $200-350 \mathrm{~m} / \mathrm{s}(656-1148 \mathrm{f} / \mathrm{s})$ due to a constant slot area positioned 
along the total blade span. This result shows a predicted lift gain of 8.99 percent at the design $C_{l}$ location, at blade station $(x=r / R)$ of 0.75 .

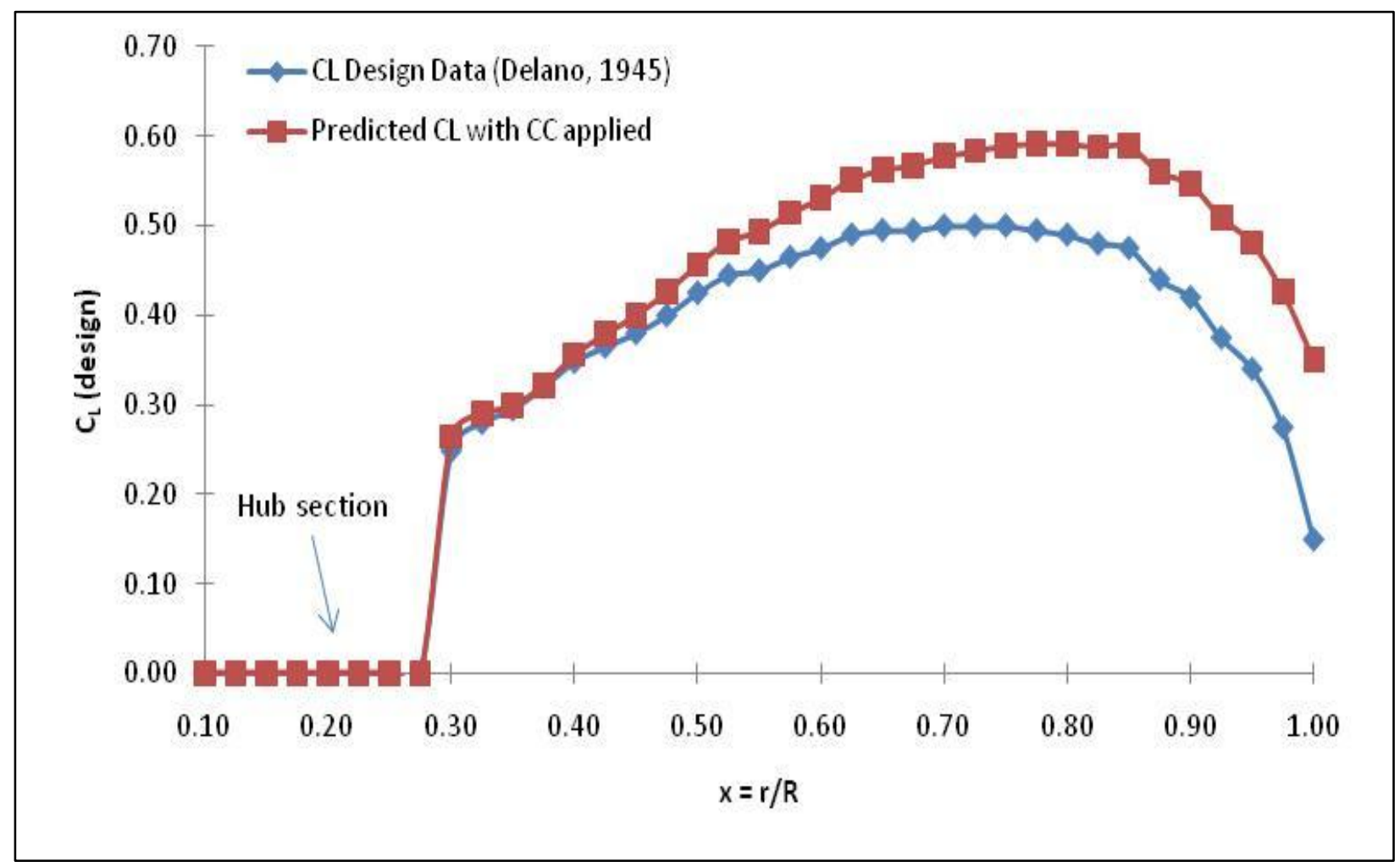

Figure 33. Predicted $C_{1}$ Increase due to Pressurization of Internal Circulation Control Plenum

\section{Computational Feasibility Study}

In order to see the effect of adding circulation control to a rotating body, such as a propeller, the aerodynamic forces were studied. Examining a propeller blade is similar to an airfoil in geometry, however, a propeller blade sees an increased angle-of-attack as the blade location approaches to the central hub. This imparts a "twist" on the propeller blade.

A computational model was built using Fluent software, with a typical grid and fluid mesh shown in Figure 34. This model, of a two-dimensional airfoil was studied to show the increase in lift across a full spectrum of angles-of-attack. For this study, a NACA 0018 crosssection airfoil was chosen, and a rounded training edge was added to simulate the Coandă 
surface. The model was built to have a span of 8 inches matching the maximum span the actual propeller has. Both the unmodified version (NACA 0018 with a rounded trailing edge) and modified version (addition of trailing edge exit jet velocity) were analyzed. A resulting velocity contour simulation of the unmodified airfoil at 15 degrees $A O A$ is shown in Figure 35, and depicts the stagnation point (red) as well as the low velocity areas toward the trailing edge of the airfoil (blue).

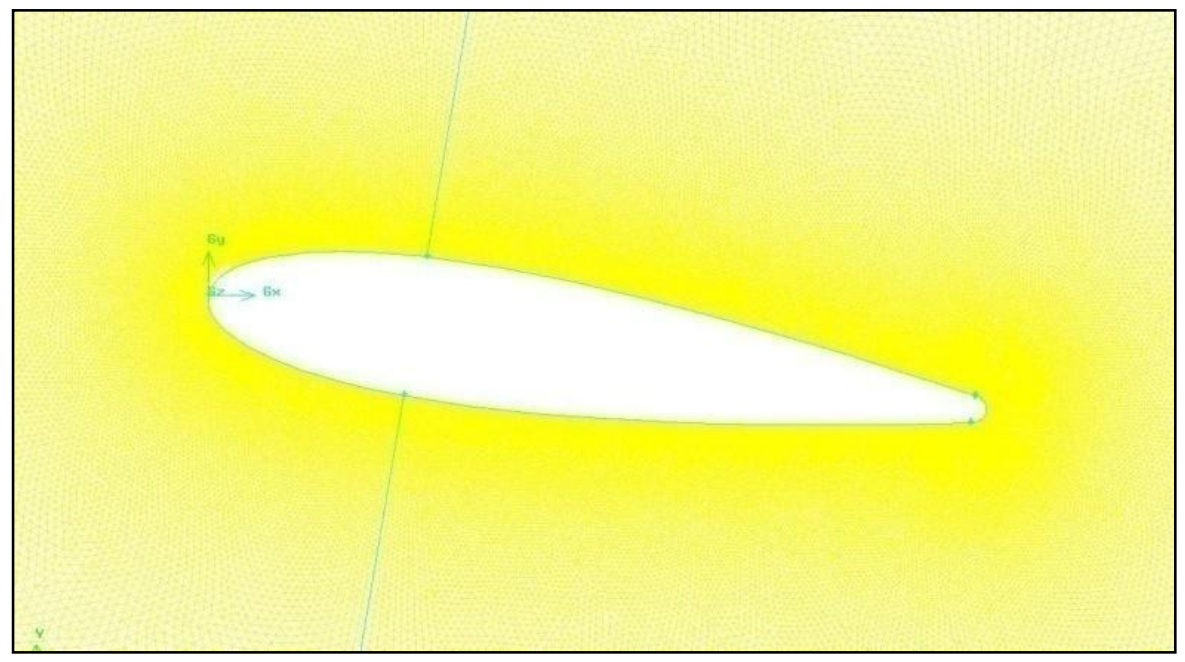

Figure 34. Fluent Airfoil Model and Mesh Spacing

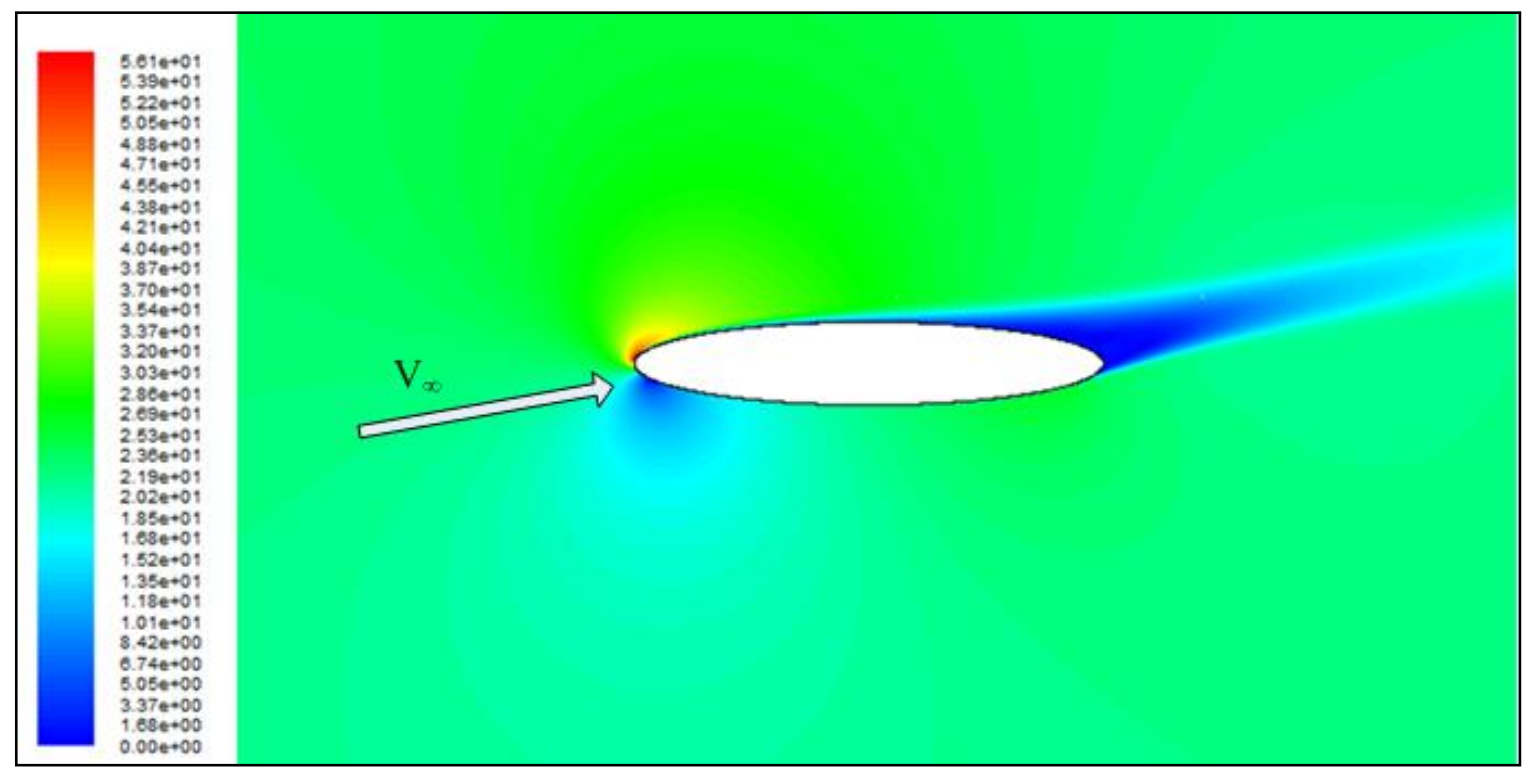

Figure 35. Velocity Contour Plot at $15^{\circ} \mathrm{AoA}$ (Unmodified Airfoil) 
The NACA 4-(5)(08)-03 two-bladed propeller has a maximum twist angle of 66.0 degrees at a radial location of $0.30(r / R)$ (Delano, 1945). This blade station is also where the propeller spinner ends and only the propeller blade experiences the free-stream velocity. The computational study was done throughout a series of angles-of-attack ranging from 0-100 degrees, in order to predict the lift increase throughout the entire radial location of the blade.

In comparing Figure 36 and Figure 37, it can be seen that the leading edge stagnation point has moved further to the underside of the airfoil for the 10 percent case. The air traveling over the airfoil for the $C \mu 0.10$ case has also increased in velocity, while the underside has decreased, illustrating the greater streamline curvature of the flow. Also seen in comparing Figure 36 and Figure 37, the trailing edge jet stays attached to the rounded trailing edge for a greater distance when the $C \mu$ increases from 0.05 to 0.10 . The longer adherence of the exit jet significantly changes the virtual camber of the airfoil increasing the $C_{l}$ generation of the airfoil (Englar, 2005).

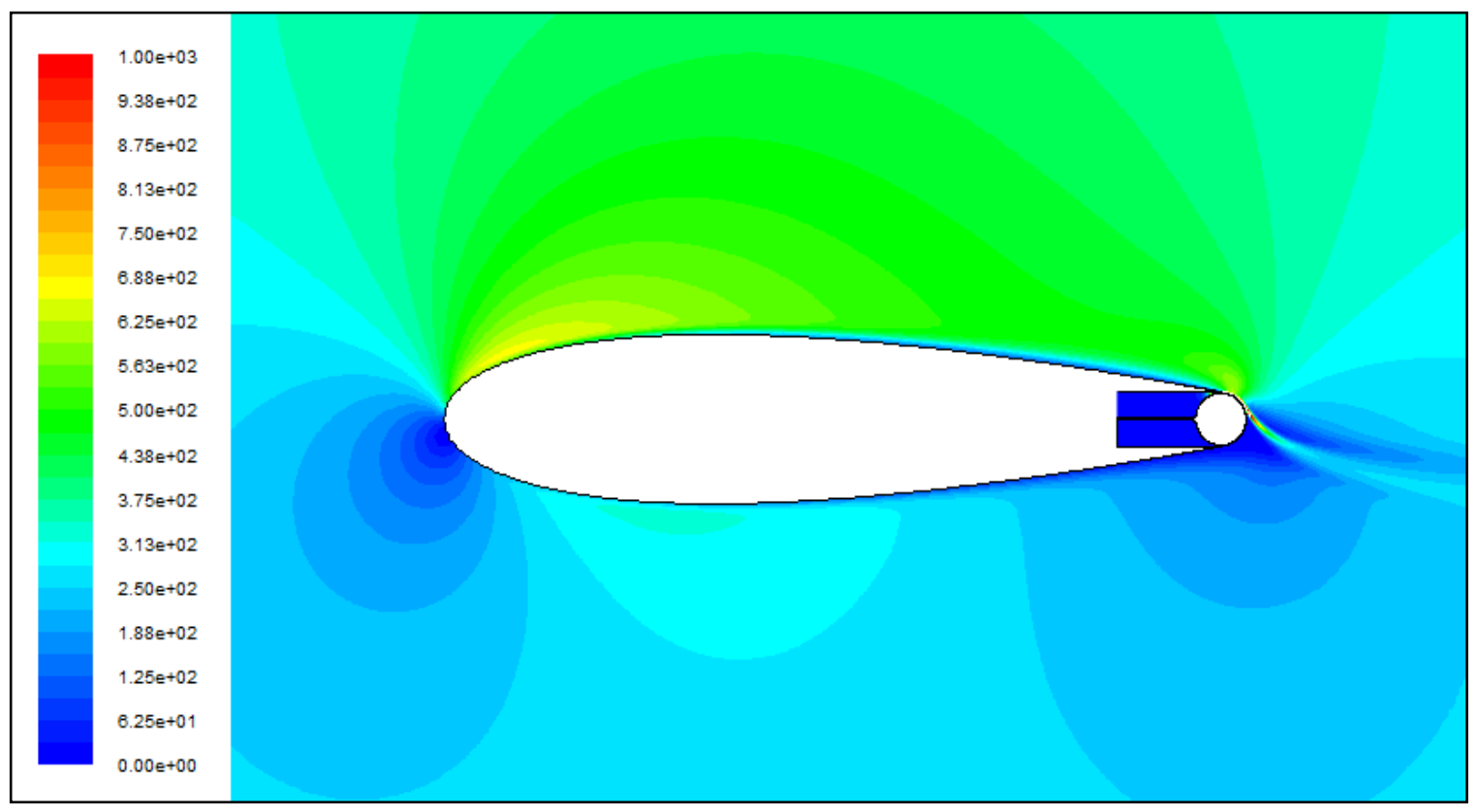

Figure 36. Dynamic pressure contours at $\operatorname{Re} 300,000, C_{\mu} 0.05$ 


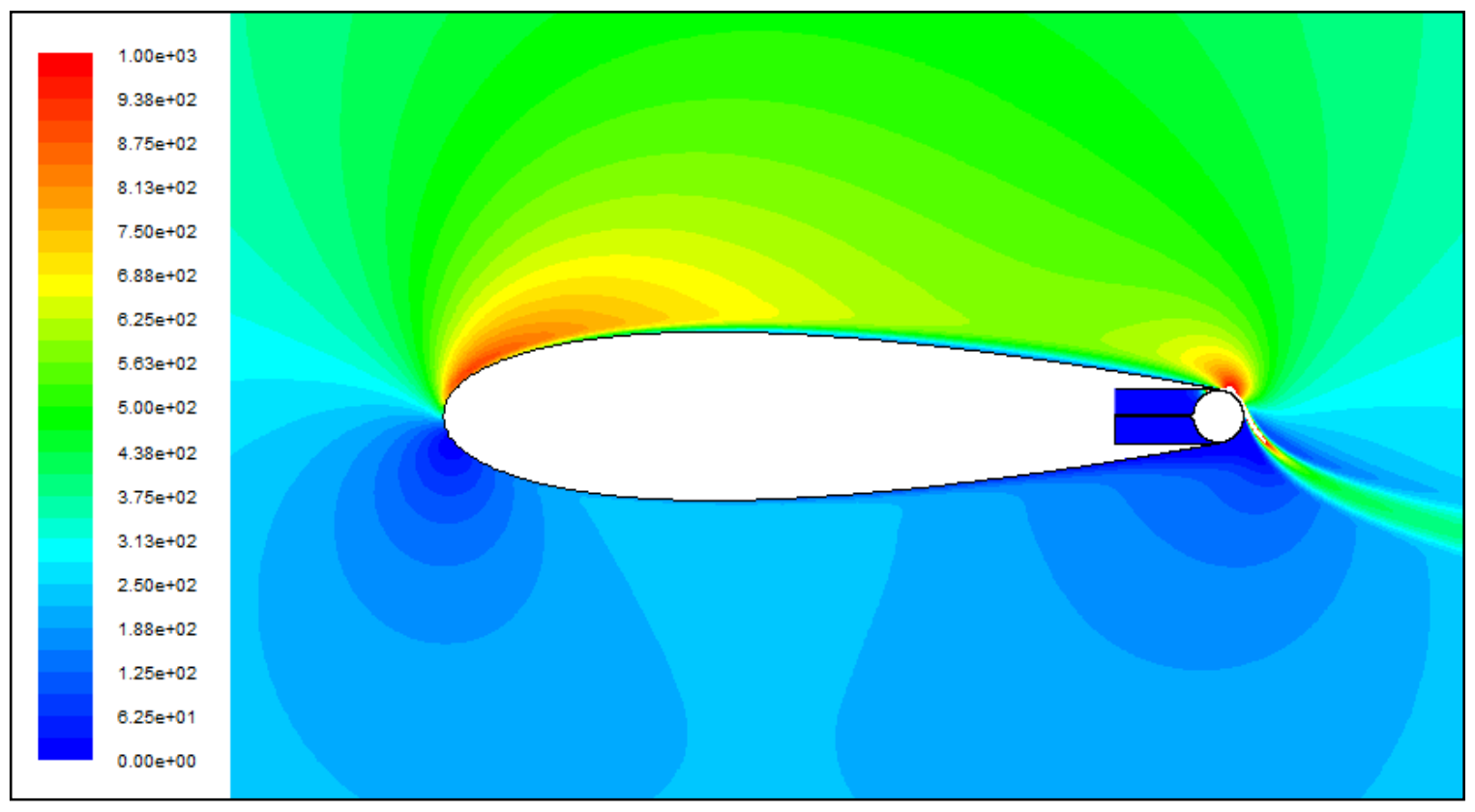

Figure 37: Dynamic pressure contours at $\operatorname{Re} 300,000, C_{\mu} 0.10$

As it has yet to be determined where the $C C$ ejection slot will be located to best augment the propeller, each available location needed to be analyzed throughout the radius. In Figure 38, the lift force profile for both the un-augmented and augmented models are shown as it corresponds to each angle-of-attack. This study was conducted at a $R e$ of 40,000 which corresponds to a free-stream velocity of 22.4 meters per second (50.0 miles per hour), at sea level. The values of 0.01 and 0.10 , corresponding to exit jet velocities of 7.59 and 38.9 meters per second (17.0 and 87.0 miles per hour, respectively) are shown on the plot below and can be seen to increase the lifting force available to the model by a maximum of 20 percent in the region of angle-of-attack the propeller blade will experience. The most effective area the $C C$ is predicted to have on the propeller blade is shown between the angles of 15-55 degrees. This suggests that the effective slot location along the propeller blade radius will be between 0.33 and $0.66(r / R)$. Although some of the dimensions of this model are ideally modeled (i.e. exit slot and chord) it illustrates an effective trend that the $C C$ technology is predicted to have on the propeller. 


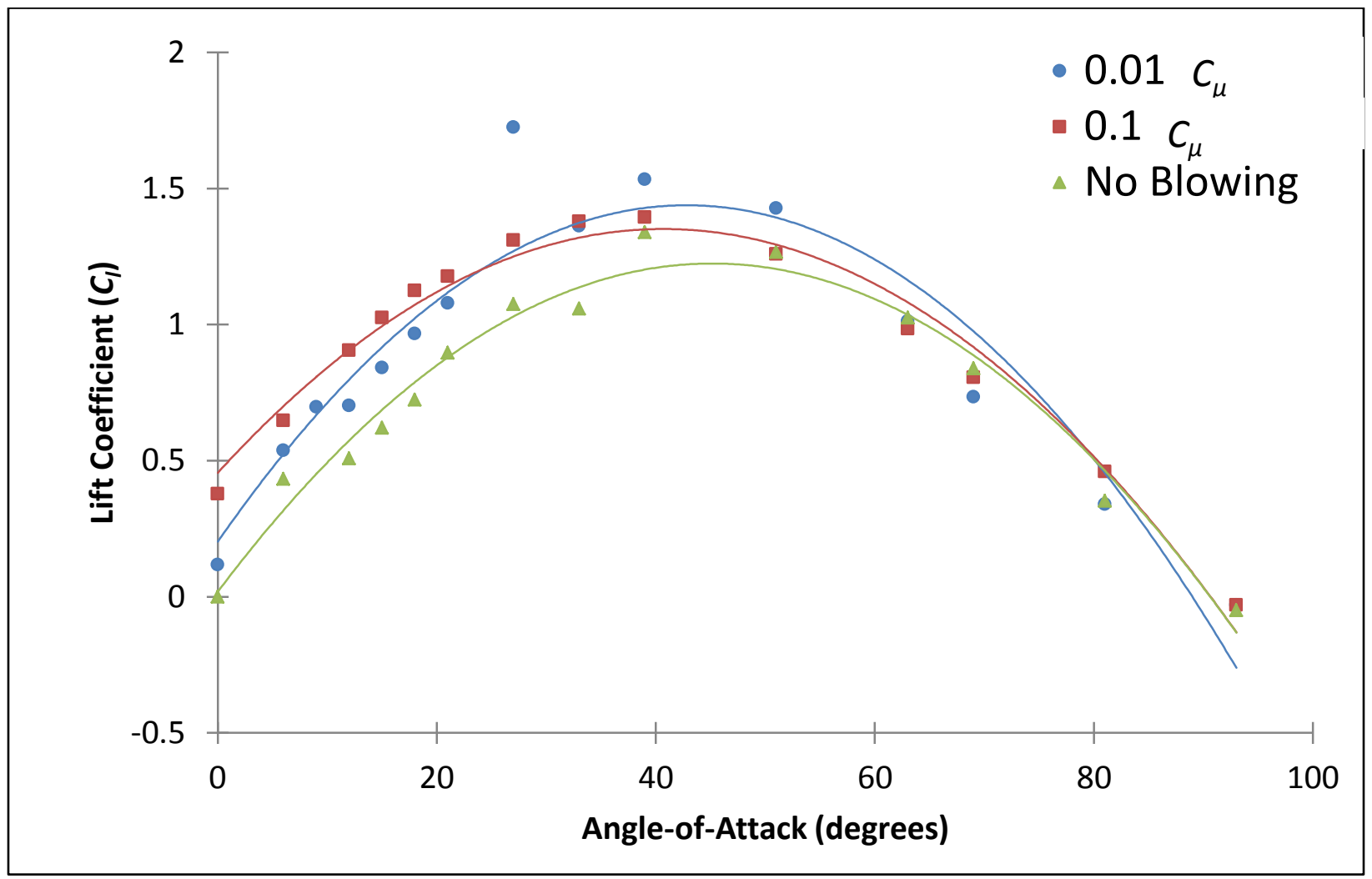

Figure 38. Computational Lift Coefficient Augmentation Prediction

\section{Summary}

It is clear that this system required a systems approach for utilization and can force a redesign of the entire propeller blade and the way it is used. Numerous studies have been conducted in the past on propellers including the use of active blowing slots to create large increases in thrust delivery. As with most active circulation control systems used, the hardware, weight, and pumping penalties far outweigh the benefits for all but a few unique applications. In contrast, and particularly regarding fuel consciousness, any passive improvements, even single digit improvements will provide value to the industry. It is believed that this technology will provide such a benefit.

Preliminary research, conducted in this sensitivity analysis, into this passive circulation control technology shows that a maximum increase in lift coefficient of 0.20 is achievable at 75 percent of the radius of the propeller blade without drastically changing any of the existing 
hardware on the aircraft. A retro-fitted propeller is currently envisioned as the only hardware change to the entire aircraft. The use of this passive circulation control system avoids the need for excess weight additions, such as pumping systems, to control the augmented jet velocity. The selection of the position along the radius and the jet exit dimensions will allow for optimization of the entire system, as well as making the exit area the limiting dimension of the system to provide consistent pressurization for the jet velocity. 


\section{Propeller Design}

Design of a testing model and apparatus can be both time consuming and expensive. In order to reduce the time and cost spent on the modeling of the experimental demonstrator, the design work was completed with the help of a three-dimensional scanner, an engineering modeling software and a rapid prototyping machine. The use of all three of these applications made the modeling faster, easier and less expensive than traditional casting and machining methods. This section will describe the process of finalizing the model with which the experimentation was completed. Figure 39 shows the process taken and tools used to create the experimental model.

\section{Choose Propeller - Purchased as Replacement Part}

\section{Zinger Model Propeller - 24 Inch Diameter}

\section{D Scan with Digitizer - Range 7 Camera}

Assemble Scans - Konica Minolta Rapidform Software
Computer Model of Augmented

Propeller - ProEngineer Software

Export .stl File

Prototyping Machine - FORTUS 900mc

Experimental Prototype

Figure 39. Propeller Design Technique

\section{Blade and Circulation Control Plenum Design}

The inside slot geometry and propeller blade plenum proved to be a difficult portion of the design of this particular propeller. The un-augmented propeller chosen for this 
experimentation was purchased as an off-the-shelf replacement part, was 0.60 meters ( $2.0 \mathrm{feet})$ in diameter and at its largest point was only 0.001 meters (0.04 inches) in thickness. After extensive background research into the use of circulation control on other airfoils, wings, and aerodynamic bodies it was found that the most important parameters for circulation control to be effective was the exit-slot-height-to-trailing-edge-radius ratio $\left(h_{j} / r\right)$, trailing-edge-radius-to-chord ratio $(r / c)$ and exit-slot-height-to-chord ratio $\left(h_{j} / c\right)$. The most useful of these parameters were plotted and can be found in the Figure 40 (Englar and Williams, 1971). The ratios of this particular propeller experiment fall just within the bounds of the region of effectiveness, with the values for this particular model being $0.04 r / c, 0.002 h_{j} / c$ and $0.05 h_{j} / r$, shown below in Figure 40, highlighted with a yellow circle, just at the upper most bound of the region of most effective Coandă operation. 


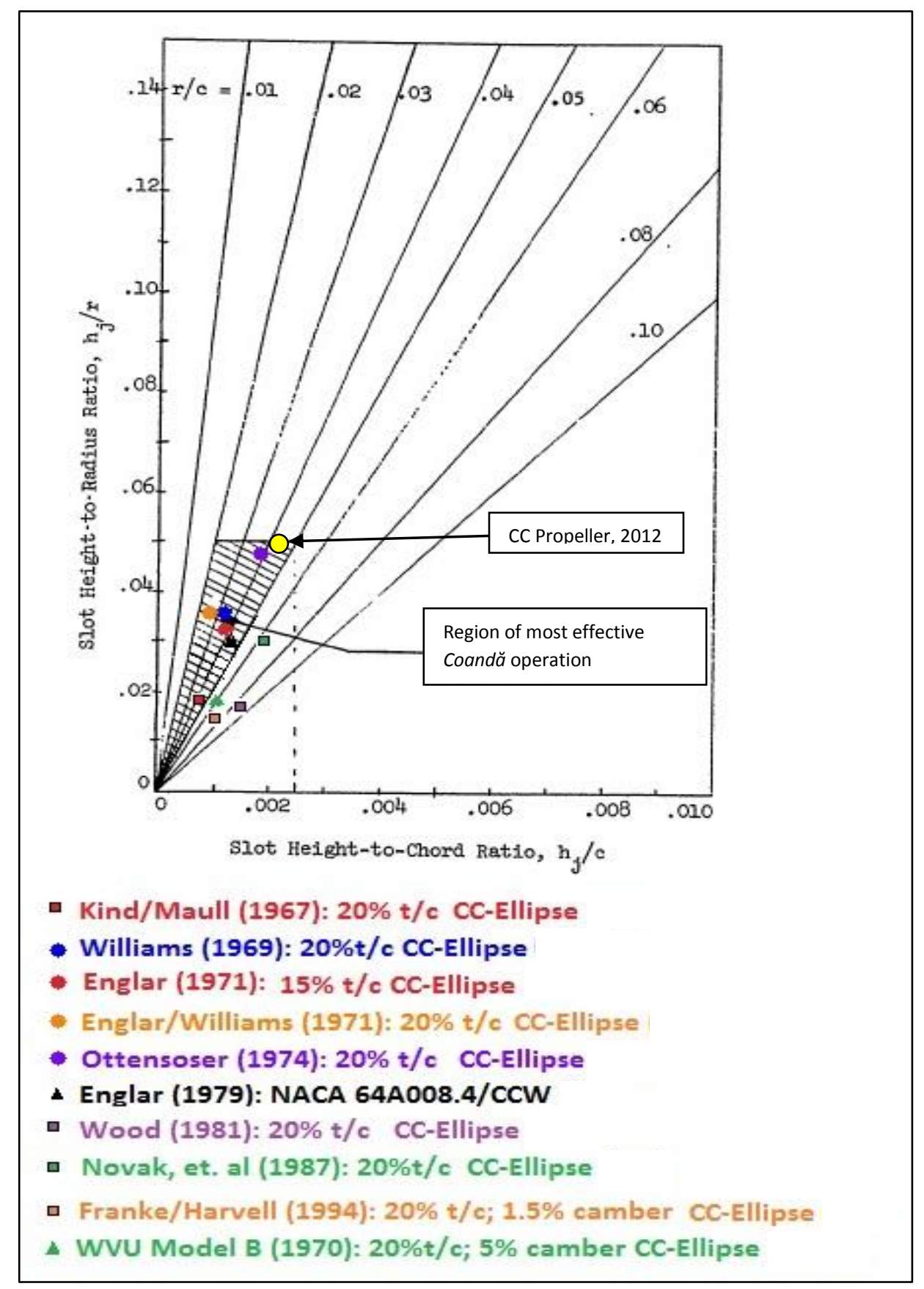

\section{Figure 40. Region of Most Effective Circulation Control Geomerty (Kweder, Panther, Smith, 2010)}

In order to model the geometry of the propeller accurately in an engineering software package, to be able to manipulate the dimensions and provide a circulation control plenum inside the propeller blades, the first step was to scan the un-augmented model with a three-dimensional camera. The scan was done with a Konica Minolta Range 7 with some smoothing and after 
processing done inside the Konica Minolta proprietary software. When the resulting model was oriented and surfaced appropriately, as a solid, with no camera artifacts, it was exported as a stereolithography file and then imported into Pro-ENGINEER. The three-dimensional modeling software aided in the ability to model the circulation control plenum to the inside of the propeller blades without the need to waste time and resources on building several different models. This software allowed for fast variation of slot geometry, and coupled with a flow visualization software the models were checked for flow criteria and a final dimension was chosen based on the flow characteristics predicted at the circulation control exit.

Through contact with the propeller manufacturer, it was found that the un-augmented propeller had a Clark-Y cross-sectional area, shown in Figure 41, with the data points fit to a unity chord length. The new augmented propeller also had a similar cross-sectional shape, less the volume of the circulation control plenum. The size of this type of airfoil illustrates the small amount of space within the propeller blades that the augmented propeller needed to be designed around. 


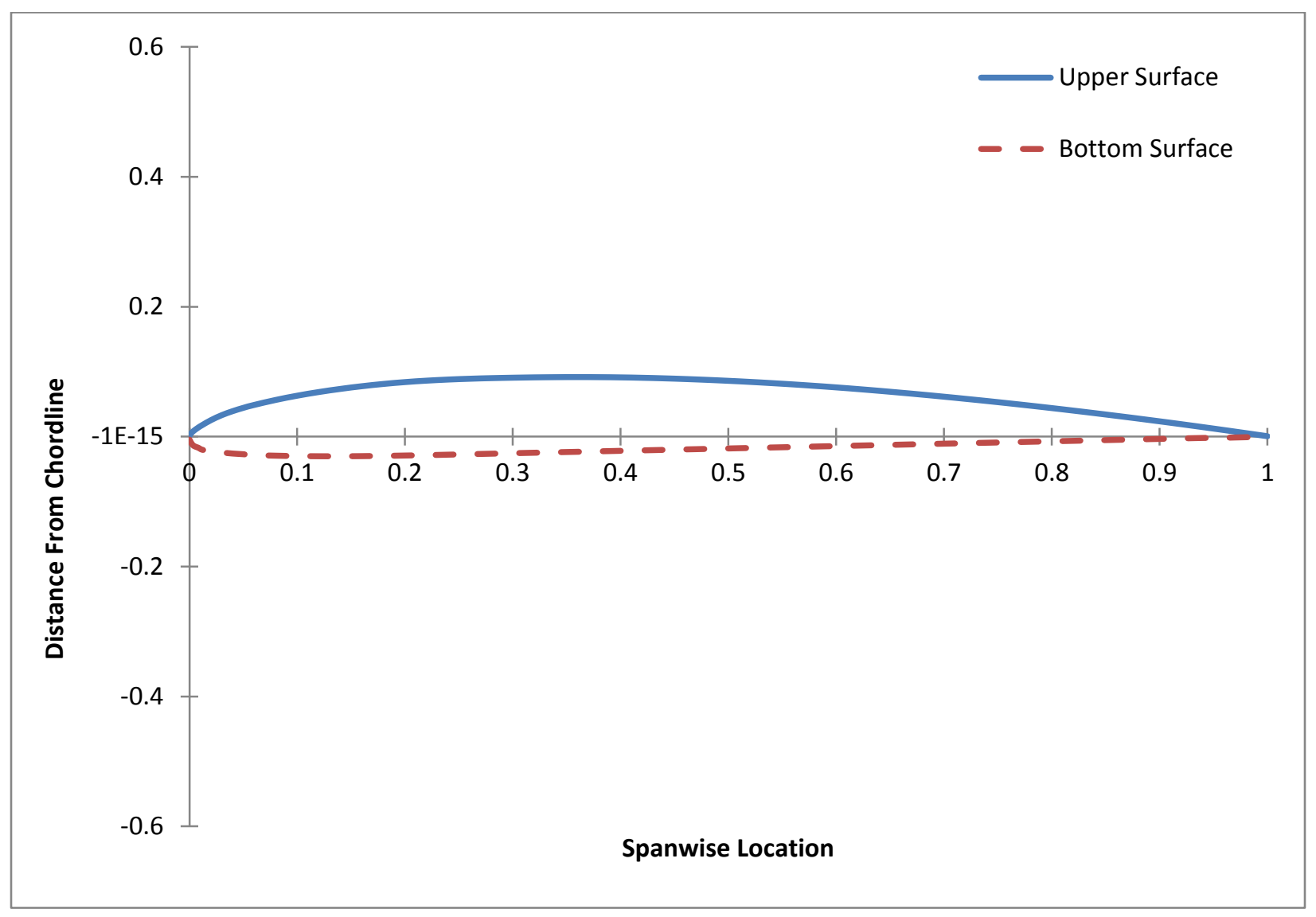

Figure 41. Clark-Y Dimensionless Cross-Sectional Area

In order to drive the airflow through the propeller blades to the exit slot, the choke area of the circulation control system needed to be the cross-section of the exit slot. The height of this slot also depended on the accuracy of the three-dimensional printer that would eventually make the prototype. The rapid prototyping machine employed to make this part was the Fortus 900mc (full specification found in Appendix B - Rapid Prototyping Machine Specifications). Taking the three-dimensional model from the computer software and modeling the propeller layer-by-layer (0.005 inches in accuracy, Hansen, 2009) two prototypes were made, one constructed of a polycarbonate $(P C)$ and the second out of ULTEM 9085 plastic. The latter is a material conceived by the rapid prototyping company and was tested to withstand bending better than the $P C$ type materials as well as having the ability to be printed in smaller layers. The ULTEM material was considered the strongest material available for the printer both in shear strength and in flexure 
resistance providing the best available choice for experimentation at high rotational and thrust loads. Below, in Figure 42, is a comparison of the original Clark-Y cross sectional geometry plotted over the $C C$ augmented chord-wise geometry. Because of the addition of a rounded trailing edge and $C C$ ejection slot, the overall chord length of the airfoil was reduced to accommodate the radius of the trailing edge.

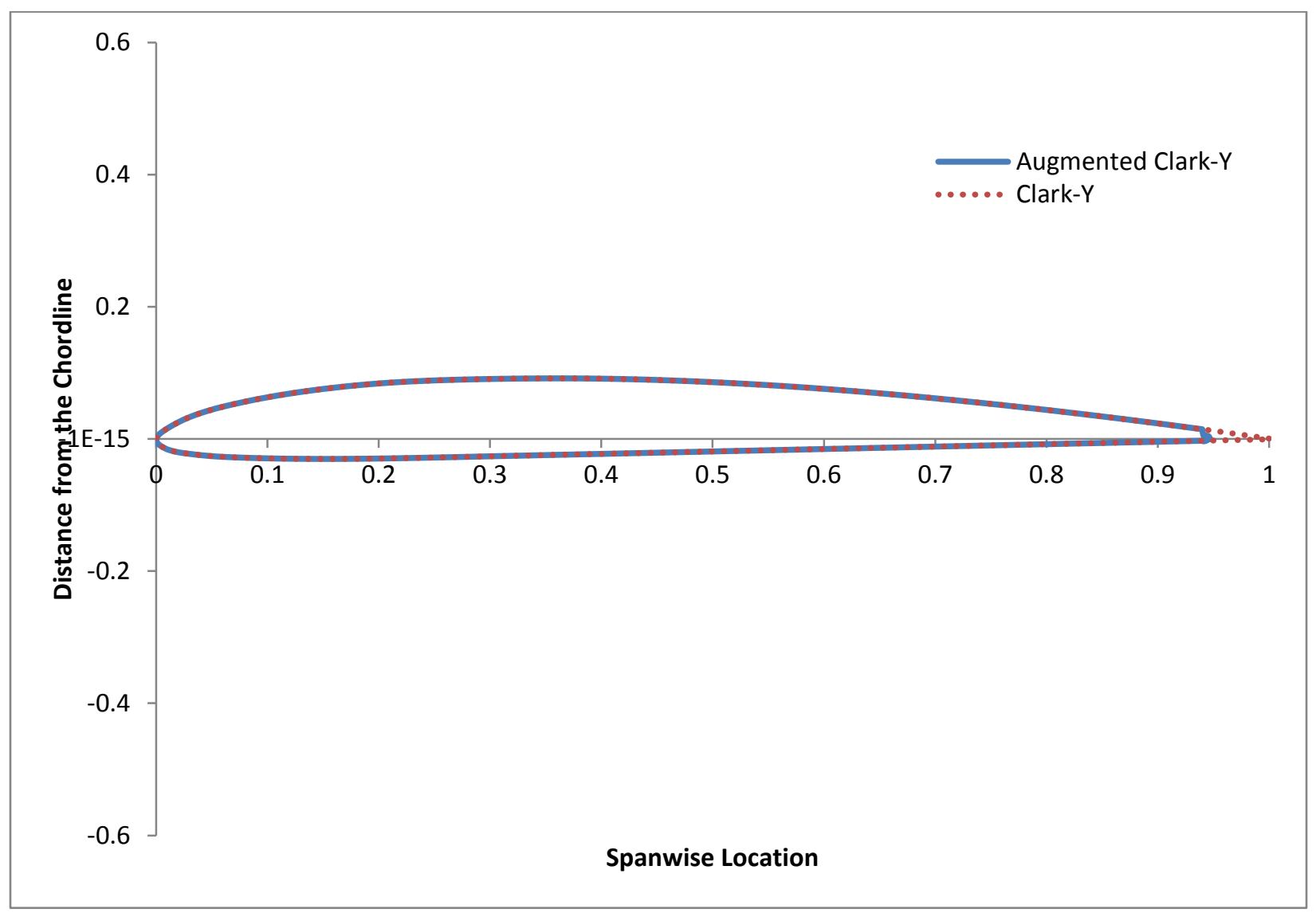

Figure 42. Rounded Trailing Edge Modification for Clark-Y Airfoil

The internal plenum of the propeller was designed in the Pro-ENGINEER software package using the Konica Minolta scanned files as a starting point. The propeller scans were all merged together and assembled as a solid model file. This model was then manipulated, using variable-section sweeps and splines to create an internal geometry to allow the pressurization air to flow from the front plane of the propeller to the trailing edge ejection slot. The geometry of the plenum had to be varied throughout the radial locations in order to allow for the twist of the 
blade and still keep the plenum relatively constant distance away from the surface walls. This was done in order to prevent any unusual bending stresses while the blade was under load while testing. Figure 43 shows the radial locations for the internal plenum slot geometry changes. In order to keep the geometry changes as uniform as possible and to avoid any abrupt crosssectional changes that would impact the airflow characteristics, there were four different sections designed (Sections A through D).

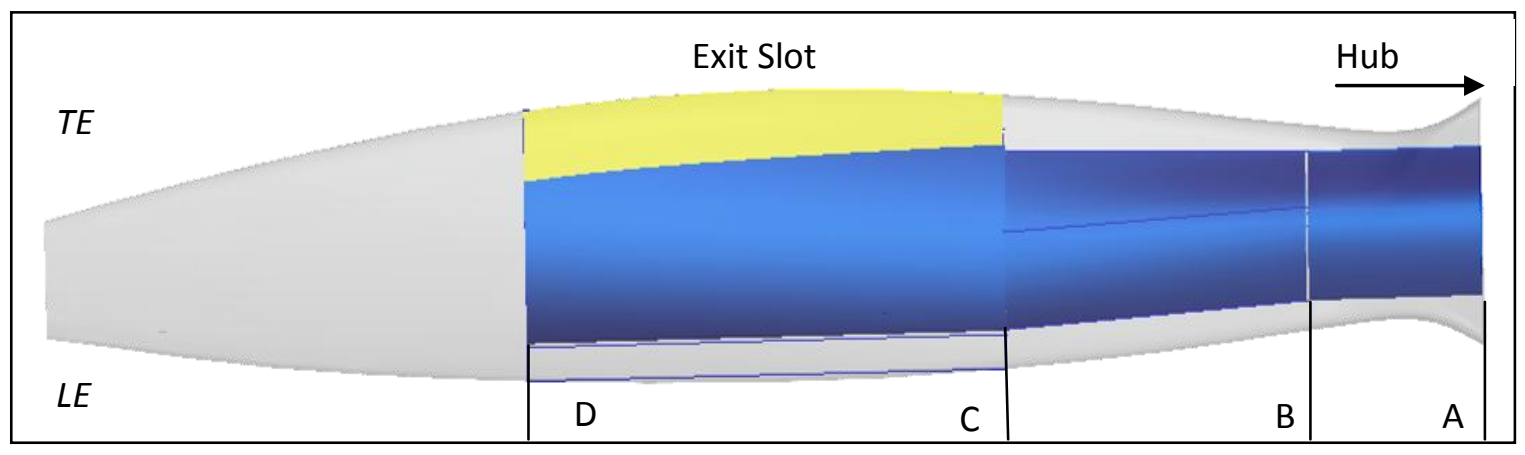

Figure 43. Radial Locations of Section Sweeps of Pressurization Plenum

In Figure 44, the root-to-tip geometry of the plenum is shown with regards to the overall dimensions of width and height. The generic shape of the section sweep did not change over the radial locations, however, as shown in Table 1, the overall width and height dimensions changed as a function of radial location toward the tip of the propeller. From section A to section B the dimensions are constant, providing a channel for the pressurization air. 


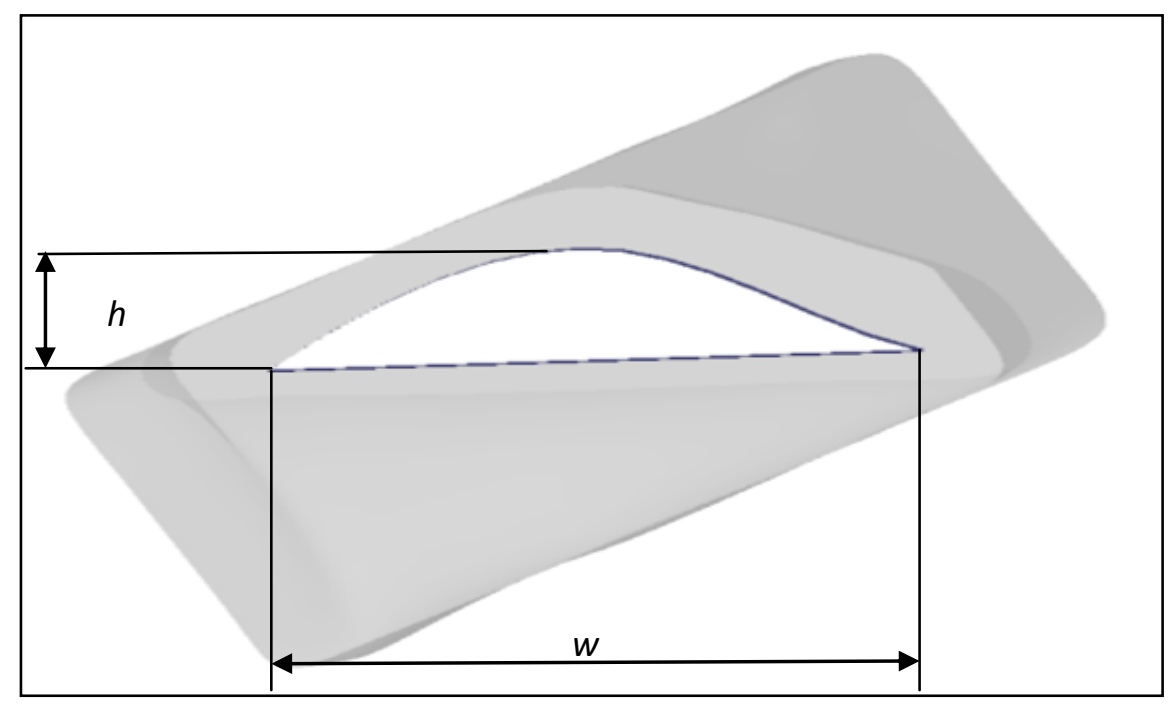

Figure 44. Height $(h)$ and Width $(w)$ of the $C C$ Pressurization Plenum

Then, the dimensions increase in the chord direction (the width of the slot) to account for the change in blade twist, forming a nozzle type of flow condition for the air to flow through. Finally between Station C and Station D, the plenum begins to reduce in size again, both in height and width dimensions, accounting for the ejection slot which spans the trailing edge of the entire 0.088 meter (3.76 inch) segment. At Station D, the plenum ends with the ejection slot, giving way to a solid blade planform as in the original propeller.

Table 1. Blade Plenum Reference Geometries

\begin{tabular}{|l|l|l|l|l|}
\hline & A & B & C & D \\
\hline Width (in) & 1.04 & 1.04 & 1.18 & 1.12 \\
\hline Height (in) & 0.25 & 0.25 & 0.17 & 0.16 \\
\hline Width (m) & 0.026 & 0.026 & 0.030 & 0.028 \\
\hline Height (m) & 0.0063 & 0.0063 & 0.0043 & 0.0041 \\
\hline
\end{tabular}

\begin{tabular}{|l|l|l|l|l|}
\hline Segment & A-B & B-C & C-D & A-D \\
\hline Length (in) & 1.35 & 2.38 & 3.76 & 7.49 \\
\hline Length (m) & 0.034 & 0.061 & 0.096 & 0.19 \\
\hline
\end{tabular}

The sensitivity analysis done on a computer model of the circulation control propeller showed that the middle third of the propeller blade provides the most useful enhancement to the output lift/thrust of the propeller. Therefore in order to minimize the effect of friction drag when 
adding a circulation control slot and rounded trailing edge to the propeller, only the middle third of the propeller was retro-fitted with cc ejection slots. In the following image, Figure 45, the exit slot of one propeller blade can be seen as well as the plenum cross-section throughout the length of the blade. To enhance the strength of the propeller as best as possible, the circulation control plenum was not extended past the ejection slot providing a solid blade cross-section along the outer third of each blade.

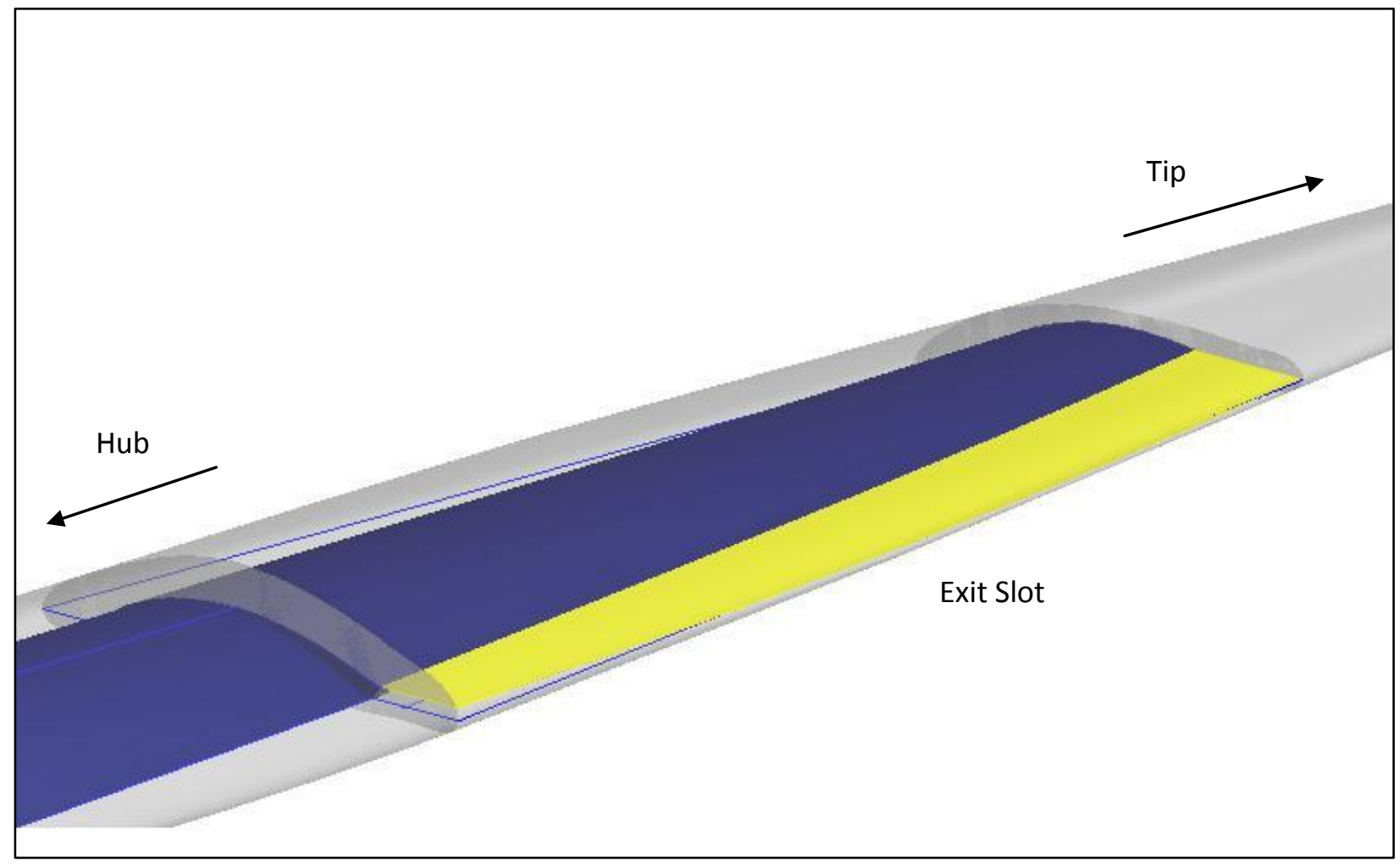

Figure 45. Exit Slot and Plenum of Circulation Controlled Propeller Blade

Several preliminary experimental rotational studies were conducted to see what kinds of stresses and displacements were predicted while the blades of the propeller were turning during the wind tunnel tests. This data was used to get a feel for what types of speeds that the propeller would be able to handle without failing due to rotational stresses during testing. As stated earlier, for ease of design and construction, and in order to prove that the physics in employing an augmented propeller, a small wooden model propeller was chosen from which to base the design. 
As this un-augmented propeller was made of a single piece of wood and the augmented propeller was printed in a three-dimensional plotter, it was assumed from the start that the augmented propeller would not be able to withstand the same rotational speeds before failing as the wooden propeller would. In conducting this rotational analysis, it was anticipated that failure of the augmented propeller would occur between 6000 and $7000 \mathrm{rpm}$. According to the manufacturer of the un-augmented propeller, this was $2000 \mathrm{rpm}$ less than the solid wooden propeller used for the original design (Zinger, Inc., 2010). Predicted Von Mises stress concentrations at 7000 rpm rotational speed can be visualized in Figure 46 and is shown to most readily affect the inside section of the propeller blade closest to the center hub.

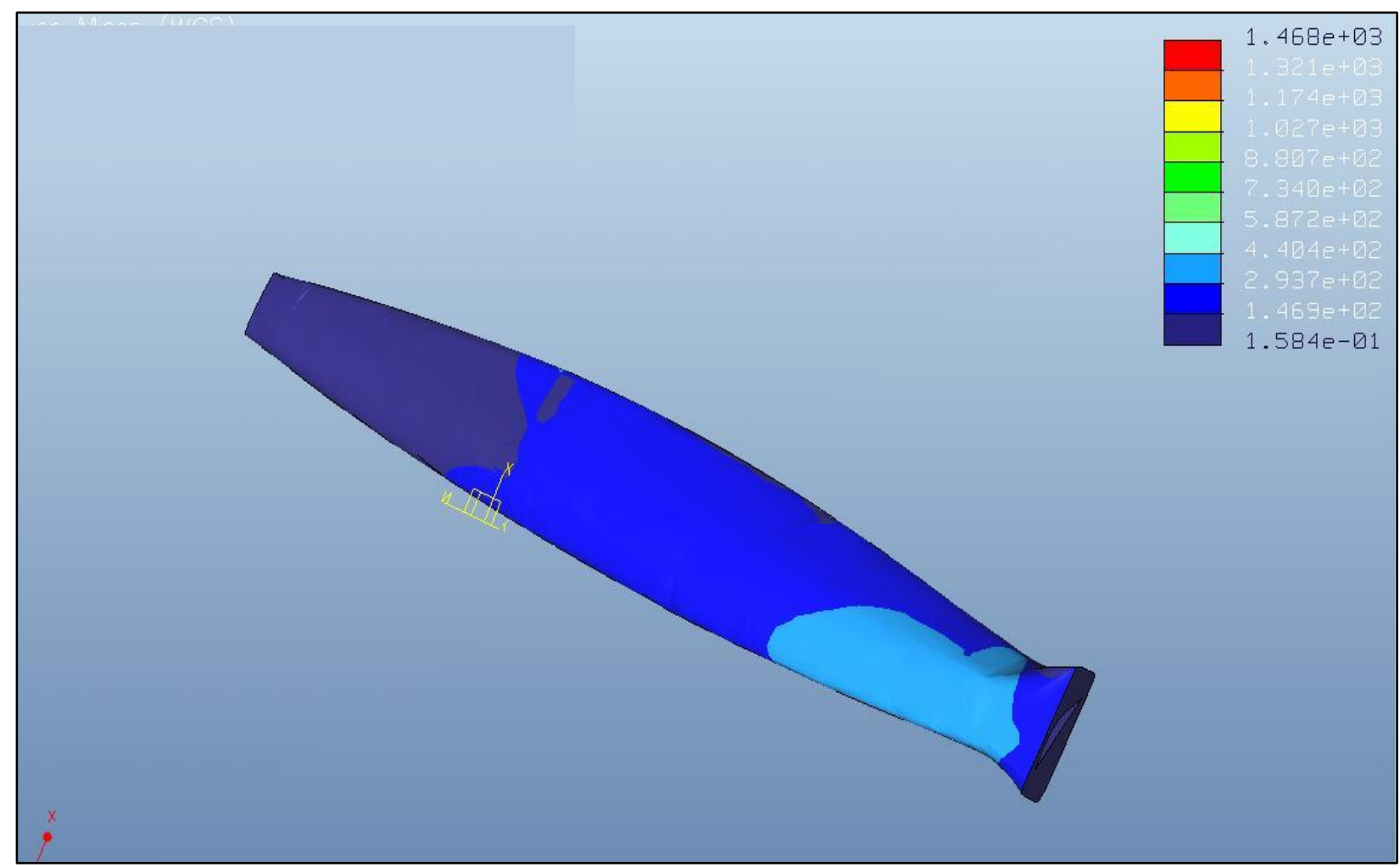

Figure 46. Predicted Von Mises Stress Concentrations at 7000 rpm

Predicted physical deformation of the propeller blades at a rotational speed of $7000 \mathrm{rpm}$ is shown in Figure 47 and can be seen to increase toward the tip of the propeller. A deformation of 0.0015 inches is predicted around the slot geometry, while the plenum is not pressurized. This 
deformation was the largest predicted at $7000 \mathrm{rpm}$. In Figure 48, an increased detail of the slot geometry and predicted deformation is shown. This concentration of deformation is due to the slot geometry and lack of support structure inside the plenum of the propeller.

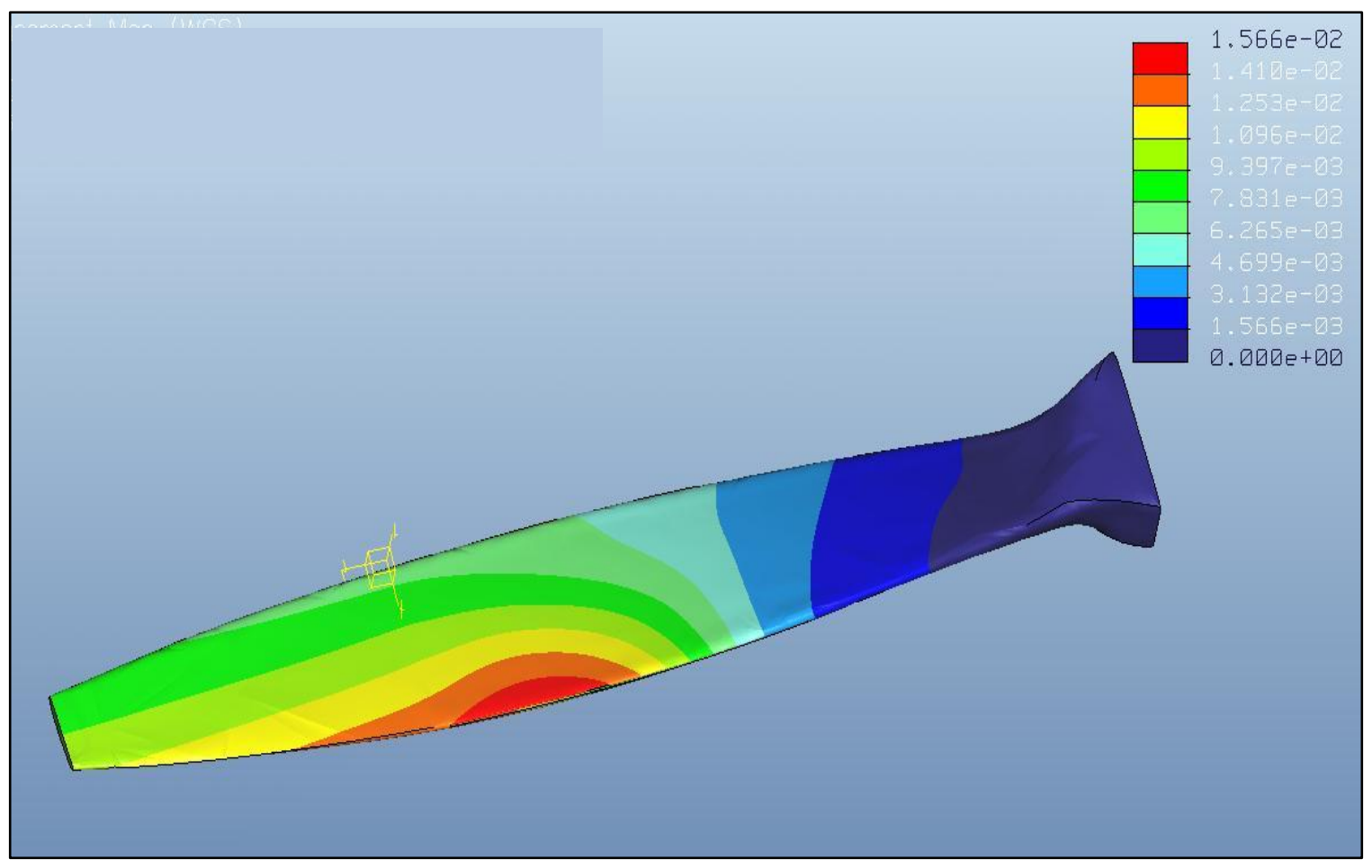

Figure 47. Blade Deformation at $7000 \mathrm{rpm}$ 


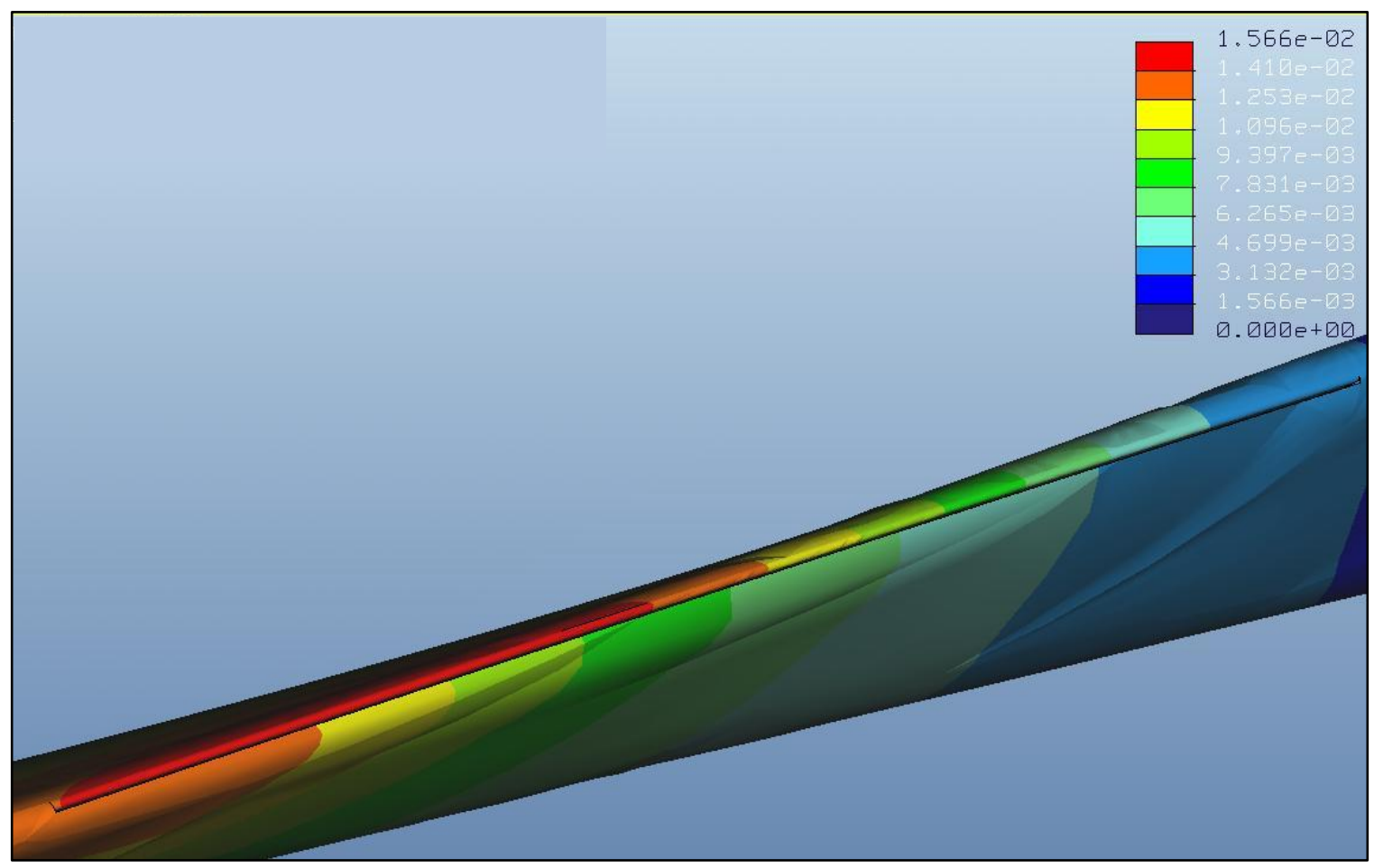

Figure 48. Increased Detail of the Predicted Circulation Control Exit Jet Deformation

Further detail and rotational studies are given in Appendix D - Rotational Study of Augmented Propeller, and show similar results with the maximum displacements being predicted around the exit slot, because of the lack of support material inside the plenum. The maximum stress concentrations are found just above the mating point between the central hub and the propeller blades, the least aerodynamic point on the propeller blade.

\section{Hub and Inlet Design}

Another important component of this proposed design is the hub-centrically mounted flow capture device, as shown along with the propeller blades as a center hub and shaft attachment in Figure 49. The inlet to the pressurization plenum lies directly upstream of the propeller surfaces and remains similar in dimensions to the hub of the original un-augmented propeller. This additional component does not add any additional drag forces to the aerodynamic performance of the propeller, as the original design used a flat section on the propeller hub. This 
allows for fast and efficient removal of the non-augmented propeller system and replacement with an augmented propeller on already existing hardware and mounting apparatus of the aircraft in question.

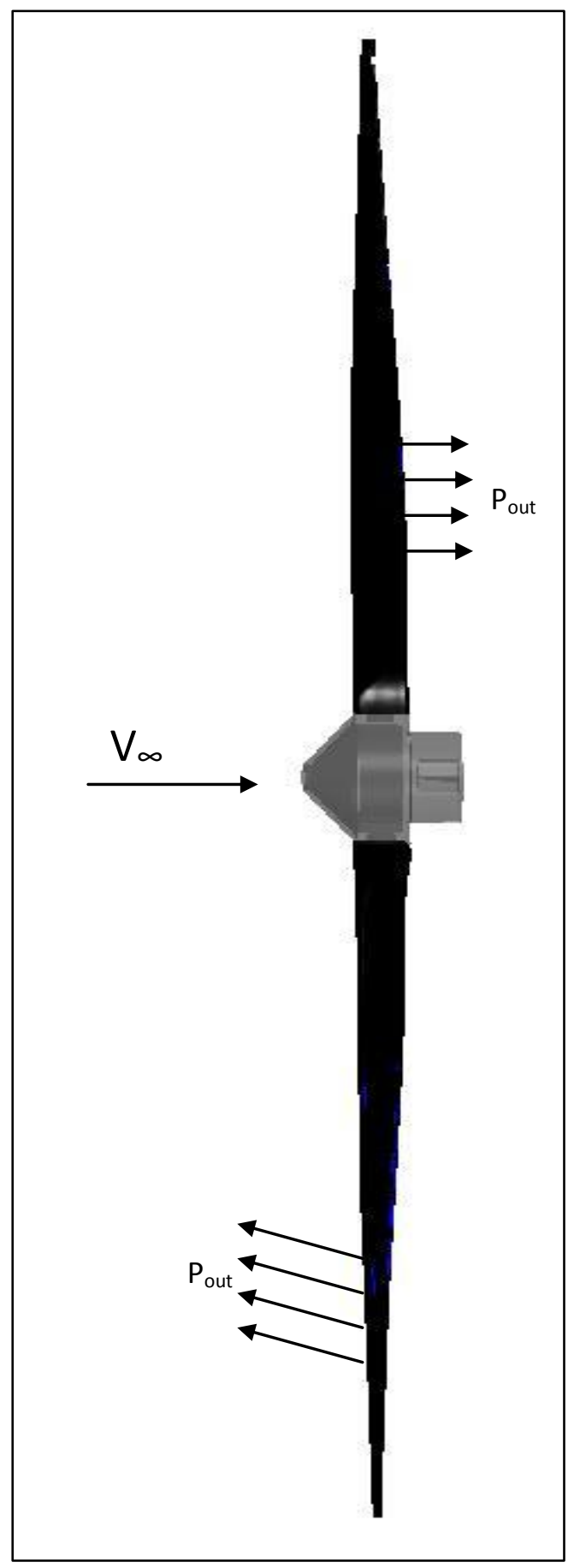

Figure 49. ProEngineer Representation of Circulation Control Augmented Propeller 
When designing the propeller hub it was necessary to have a shaft coupling attached directly to the central hub in order to be able to place the propeller on a motor-shaft linkage for experimentation in a wind tunnel. This hub was designed to be smaller than the frontal propeller spinner and pressure capture device to reduce the amount of effect it had on the wind tunnel airflow. With the hub made as a part of the propeller, the forces from the rotating propeller were easily transferred from the rotating shaft and electric motor through the propeller without the need for bolts and attachment points to hold the loads. Figure 50 shows a close up view of the propeller hub and pressure capture device for the front surface of the propeller, with the shaded areas representing the hidden hollow parts inside the model. It is shown that the forward velocity gets captured in the opening in the front surface of the conical spinner and pressurizes the inside plenum of both propeller blades directly with the help of rotational and centripetal forces acting while the propeller is in motion. The pressurized air then flows through the propeller blades until it finds the exit jet area at the circulation control slot on the outer rear surface of the blades. This is where the flow leaves the plenum and as predicted, re-energizes the boundary layer, thus, enhancing the overall lifting forces of the blades with the addition of the Coandă Effect. 


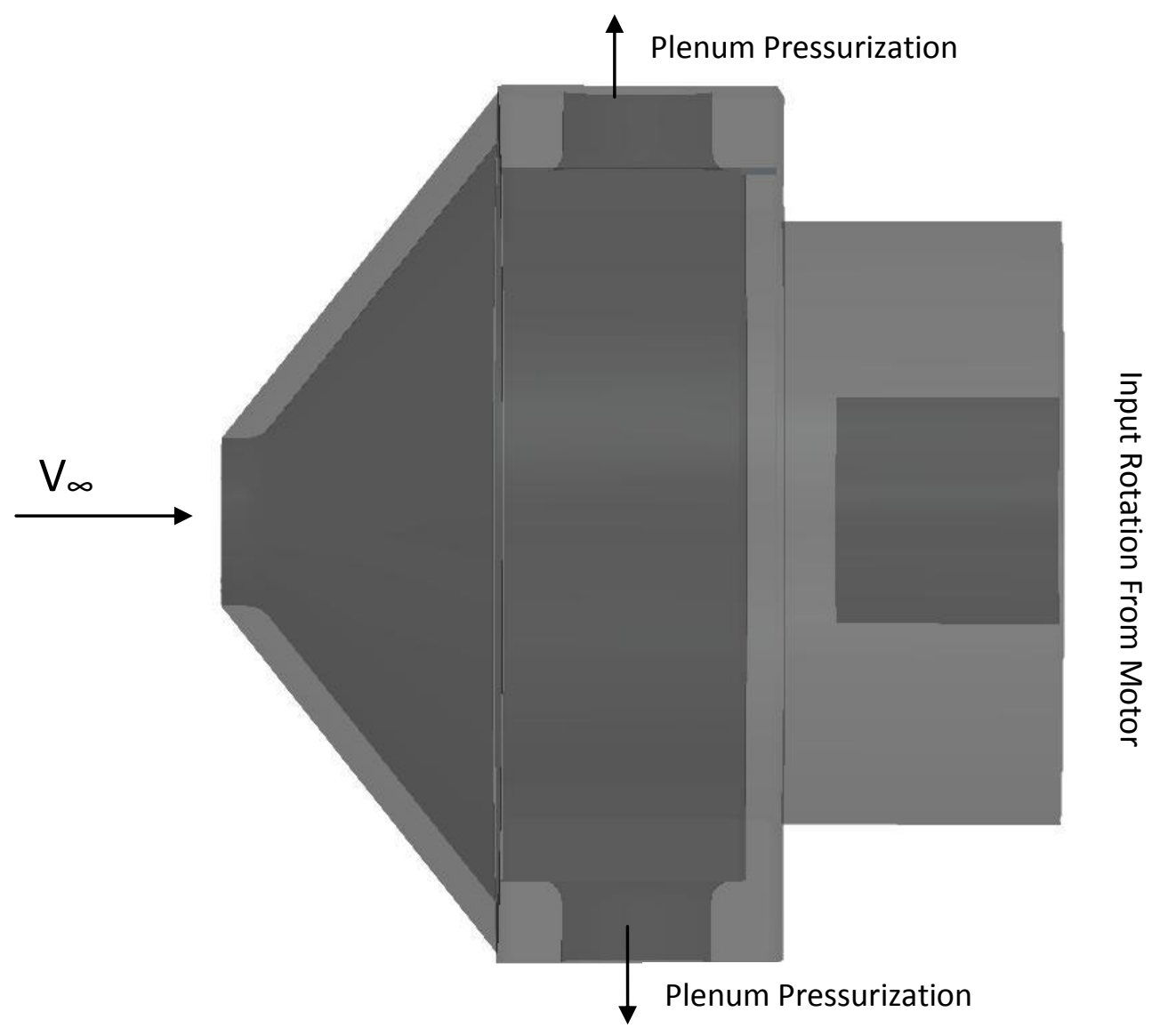

Figure 50. Fundamental Design of Capture Apparatus (not to scale)

\section{Summary}

Using computational modeling as well as some simulation software analyses in Fluent CFD solver and Pro-ENGINEER three-dimensional modeling tools, it was possible to simulate several different designs of an augmented propeller design based on theoretical input variables and fluid mechanics. Without needing to build and experiment on multiple models in a guessand-check manner, it was possible to save some program costs on the propeller model by only needing to build the final experimental geometry needed to continue with this study. 


\section{Experimentation}

The overall goal of this experimental plan was to provide proof-of-concept to a new form of application of circulation control and provide the physical evidence as well as begin a generalized computational model which allows for parameter variance when attempting to optimize the systems based on propeller duty cycles and flight envelopes to be used later in this research program. The model could then be used in a research capacity in other fluid mediums and optimize the same system for both compressible and incompressible fluids.

In order to properly study the effects of this additional system on a propeller, a computational fluid model was developed, and a two foot diameter propeller was analyzed both with the $C C$ active and inactive. In conjunction with the computational model, a physical model of the $C C$ propeller was constructed and tested in a wind tunnel environment.

When correct data was produced, in accordance with the historical lift and drag data provided, the assumption was made that the computational model was accurate and could be used to verify other model propellers. This computation model was used to study the effect that the exit jet velocity has on the augmented propeller and what the optimal dimensions of this exit slot are for $U A V$ employment.

The next step in the experimentation was to take wind tunnel measurements of the lift and drag forces, in order to compare them to a computational model. With a comparative value from the experimental and computational results, this experiment provided a usable predictive modeling tool to apply passive circulation control technology to other propellers and rotating bodies for use in optimization of the system on a per application basis. Measurement systems (green) and corresponding measurement values (purple) are shown in Figure 51, detailing each variable (orange) used during experimentation. 


\section{Propeller Experiment}

\section{Pre Process}
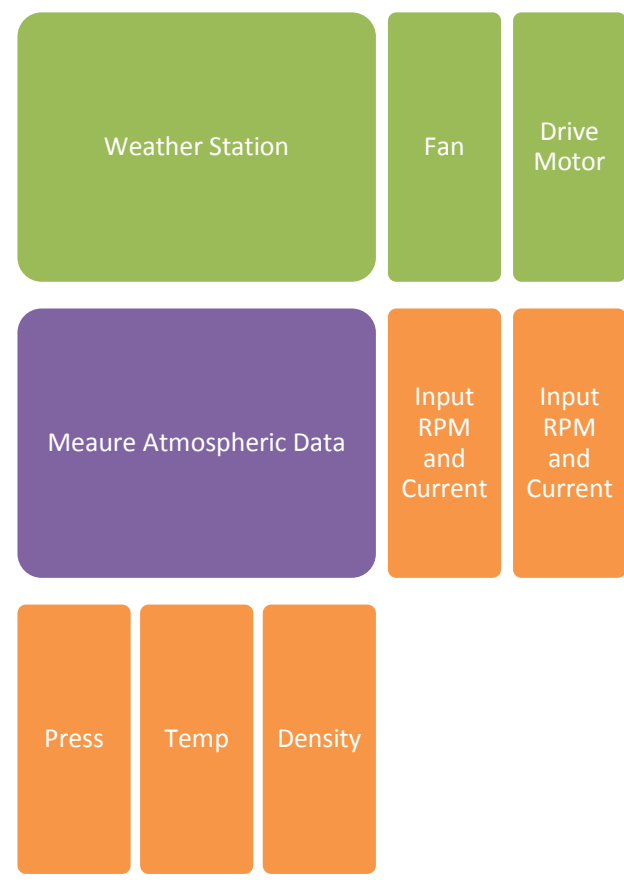
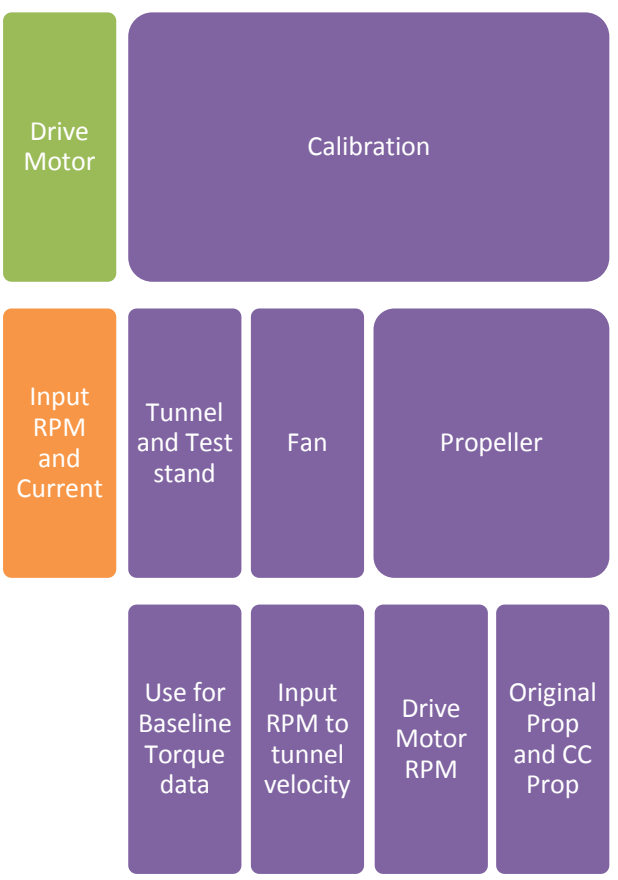
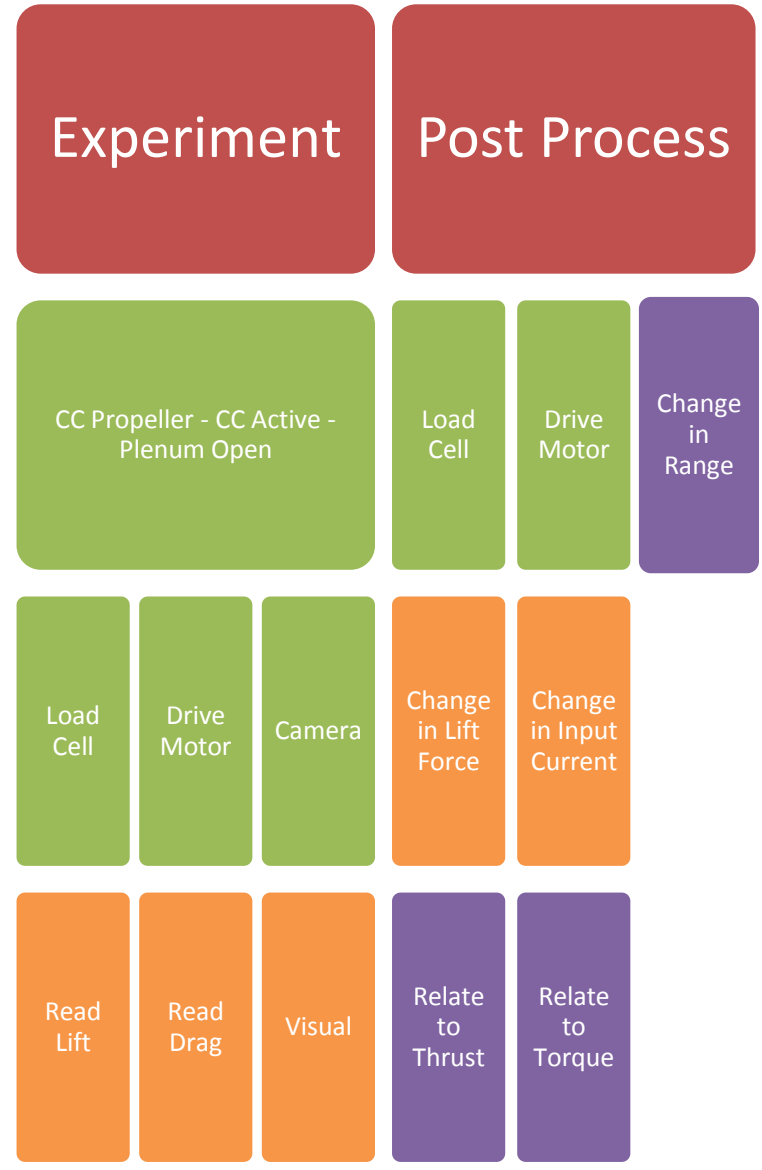

\section{Figure 51. Experimental Flow Chart of Measurable Data and Testing outputs}

In order to have validation cases for comparison to the computational models, an experimental set up, in a wind tunnel, or similar environment, needed to be conducted. Accurate readings of lift, drag, and thus thrust needed to be measured over a variety of different wind speeds and rotational speeds. These measurements were interpreted without dimensional units in order to compare the data over a large spectrum of input data and to be useful in future research applications. The results of the testing scenario were then compared to computational fluid 
dynamics results with similar set-up and boundary conditions in order to get a workable model in place for predictive purposes.

With an agreement between computational and experimental results, the other dimensional inputs of the propeller wing section can also be varied via future experimentation in the same situational set-up to see what types of effects the twist, blade taper, chord length, and cross-sectional airfoil geometry have on the measured values of lift, drag, and output torque. The overall idea of working two separate testing methodologies (analytical and experimental) is to gain confidence in the computational modeling set-up in order to be able to effectively change physical propeller and atmospheric parameters without having to build new physical testing models over and over again.

\section{West Virginia University Experimental Apparatus}

A variety of facilities as discussed in previous sections could have been used for testing this particular prototype. These experimental environments were all considered based on the size of the test section available to accommodate a model, the velocity constraints available in freestream testing, and the availability of testing and data acquisition resources. The methods readily available were:

- West Virginia University ( $W V U$ ) Closed-Loop Wind Tunnel,

- A small-scale water tunnel at $W V U$,

- WPAFB/AFRL Vertical Wind Tunnel, in Dayton, Ohio,

- NASA-Langley Full-Scale Tunnel, Langley, Virginia

- NASA-Ames Full-Scale Tunnel, Mountain View, California

- Build a new model-specific wind tunnel, WVU Hangar, or

- Mount the prototype to a sting and test the propeller via static loading only 
It was also not feasible to conduct this experiment in the West Virginia University Closed-Loop Wind Tunnel because of the blockage ratio that the model would have produced and consequent velocity reduction in the tunnel head while studying. For similar reasons, the use of the $W V U$ small-scale water tunnel was also less than ideal. While static loading of the propeller could potentially provide reliable results to compare to historical data, it did not provide the opportunity to see what the effect forward velocity and free-stream pressurization provided to the propeller model. This type of testing would be solely relying on the suction forces of the propeller disk to pressurize the plenum for circulation control. Also ruled out was the NASA-Langley facility and the NASA-Ames Facility due to cost constrains of renting the tunnel and building a model large enough to be accurately experimented on in either of these tunnel environments.

One of the feasible experimental test apparatus was to build a completely new wind tunnel environment specifically designed around the propeller itself. For this particular study, with the availability of a Cincinnati SQAF - 270 Airfoil Blower (Specifications and dimensions found in Appendix A - Cincinnati SQAF - 270 Specifications) centripetal fan from another experiment, a new wind tunnel could be constructed. The tunnel was constructed out of two standard sheets of 16 gage steel $3.60 \mathrm{~m} \mathrm{X} 2.40 \mathrm{~m}$ (12.0 feet X 8.0 feet) rolled to a diameter of 1.16 meters (3.80 feet). This was chosen to fall within the required values of wind tunnel interference values for valid experimental results and minimal correction to the free-stream velocity (Glauert, 1943). As shown in Figure 52, the correction factor will change due to area ratios $(A / C)$ of the tunnel $(C)$ to the model $(A)$. In the case of this experiment the value remains at 0.25 for both the un-augmented and augmented propellers. The two sheets of steel were assembled together and formed a complete 7.20 meter $(12 \mathrm{ft})$ long tunnel section attached to the 
inlet of the centripetal fan. The tunnel was applied to the inlet of the fan in order to provide a suction force and ultimately to maintain a consistent testing velocity throughout the tunnel.

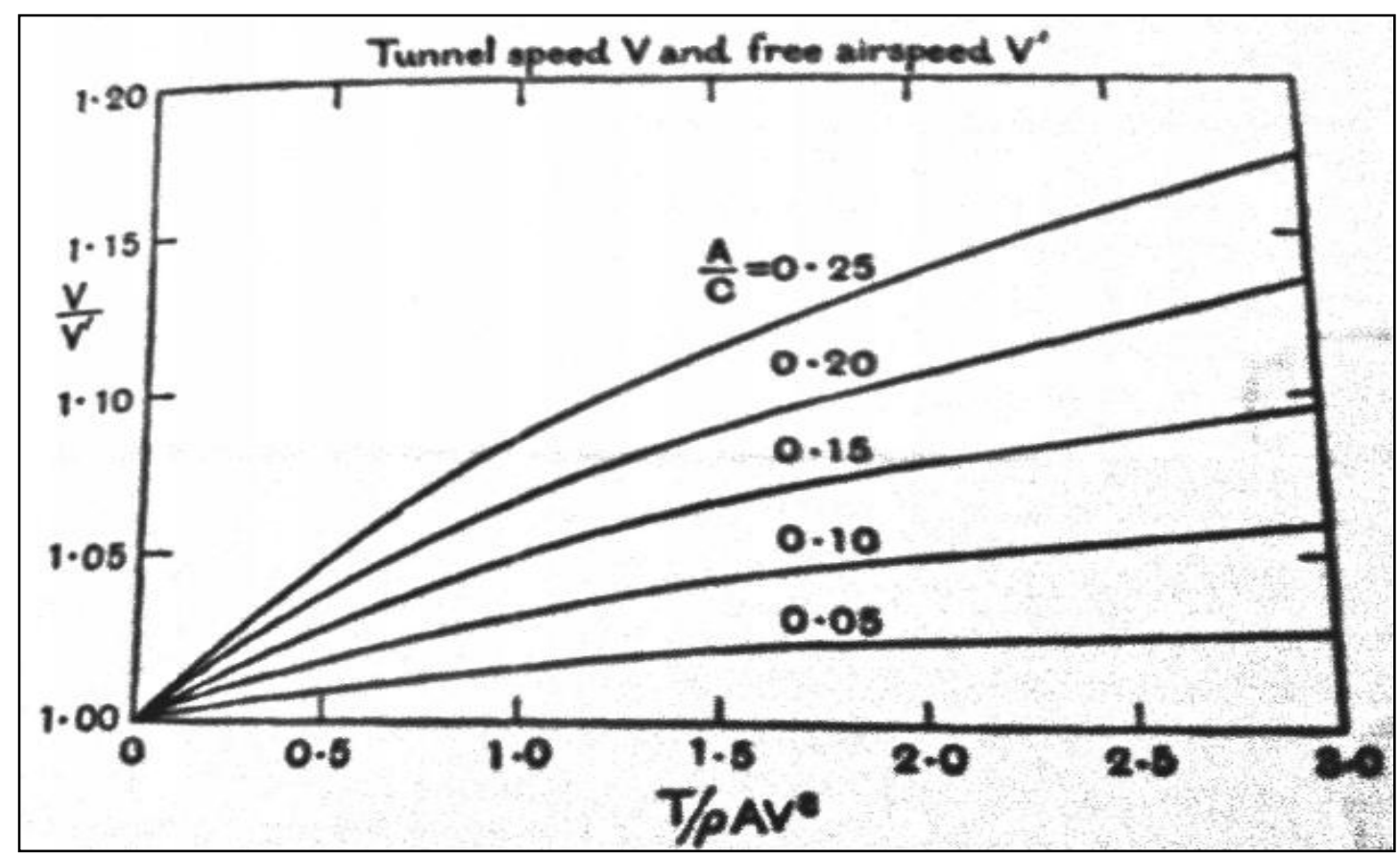

Figure 52. Free-Stream Velocity Corrections Based on Tunnel Interference (Glauert, 1943)

With the centripetal nature of the fan the exit velocity provided was too choppy and unclean to run a reliable experimental test, thus the fan inlet was used as the flow driving mechanism. The theoretically available wind velocity for this particular tunnel was dependent on flow conditions of the fan which provided a maximum volumetric flow rate-of $9.6 \mathrm{~m}^{3} / \mathrm{sec}(338.9$ $\left.\mathrm{ft}^{3} / \mathrm{sec}\right)$. This provided a theoretical velocity of $21.2 \mathrm{~m} / \mathrm{sec}(69.6 \mathrm{ft} / \mathrm{sec})$ at the fan inlet and 9.35 $\mathrm{m} / \mathrm{sec}(30.7 \mathrm{ft} / \mathrm{sec})$ at the tunnel inlet when tunnel losses were included.

This allowed for experimental studies to be conducted throughout various low speed tunnel free-stream velocities in order to analyze the effect of the circulation control slot geometry on the propeller output parameters. Specifically this study looked at the effect that the geometry had on output thrust and input torque needed at a known advance ratio. 


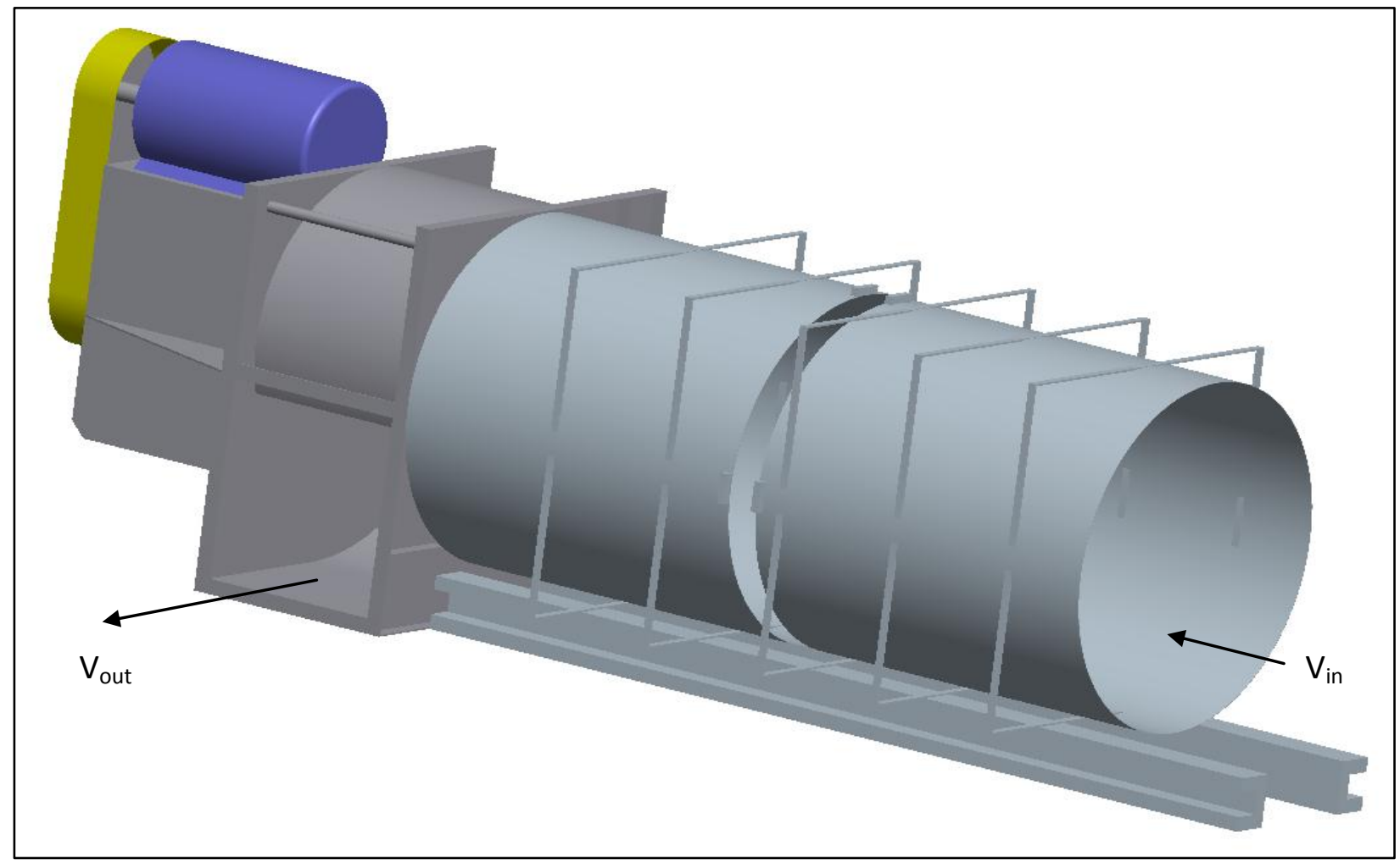

Figure 53. Wind Tunnel Assembly and Centripetal Fan, Pro-ENGINEER Model

The experimental test stand was fitted to mount the propeller inside the wind tunnel and measure the thrust output at a range of desired rpm as well as the torque required to turn the propeller. These two measurements could then be related to the lift and drag forces experienced by the propeller blades. The Thrust reading was measured directly via linear bearings which fed into a strain gauge-type load cell mounted below the shaft and motor supports.

In Figure 54, the components used to measure the output forces are shown along with their orientation in relation to the propeller. Working directly back from the propeller hardware included an aluminum plate to mount the motor on, and a linear translation bearing assembly used to transfer the thrust force from the propeller to the experimental test stand. This allowed for the propeller to "push" the entire system and the thrust force was read as an output voltage from a strain gauge-type load cell mounted on the angle supports between the mounting plates. 


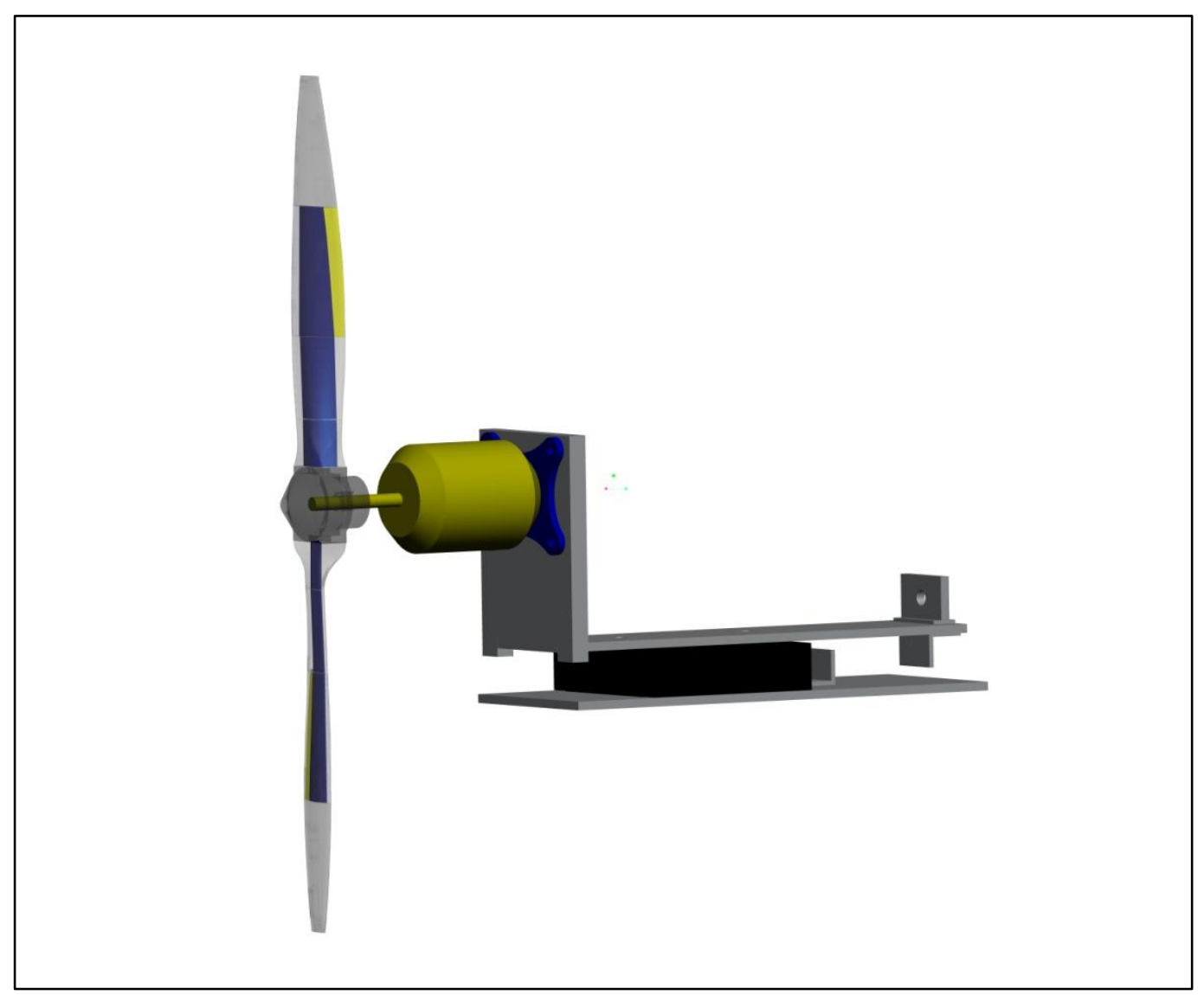

Figure 54. Motor and Propeller Experimental Test Stand Model

The torque needed to turn the propeller was the other force to be measured. This could then be directly related to the overall drag on the system. The difficulty in measuring the torque on a propeller is the simple fact that the model is in rotation and the force acts in a direction parallel to rotation. This required some alternative measurement methods to be employed. In Figure 56, the motor mount and experimental assembly is shown, and was used to measure the thrust force while the propeller is in use. On the lower section of the test stand is the electronic speed controller which was used to accurately set the speed input of the motor via servo dial input (0-100 percent input). Among the other instrumented hardware used during experimentation were time series values from a thermocouple, mounted directly to the motor coils to monitor the coil temperatures and to avoid overheating. Another value was read from an s-type load cell mounted to a linear bearing and used to validate the thrust forces experienced by 
the propeller. In order to measure an input value for the motor current, a voltage drop was read across a $50 \mathrm{mV}$ shunt which was in series just before the electronic speed controller and was then converted to an input current using Ohm's Law. The relationship between the input voltage and the read voltage drop was used to estimate the rotational speed of the propeller under load and to calculate the propeller efficiency and power requirements. The relationship is a linear trend between minimum and maximum voltage input values, shown below in Figure 55.

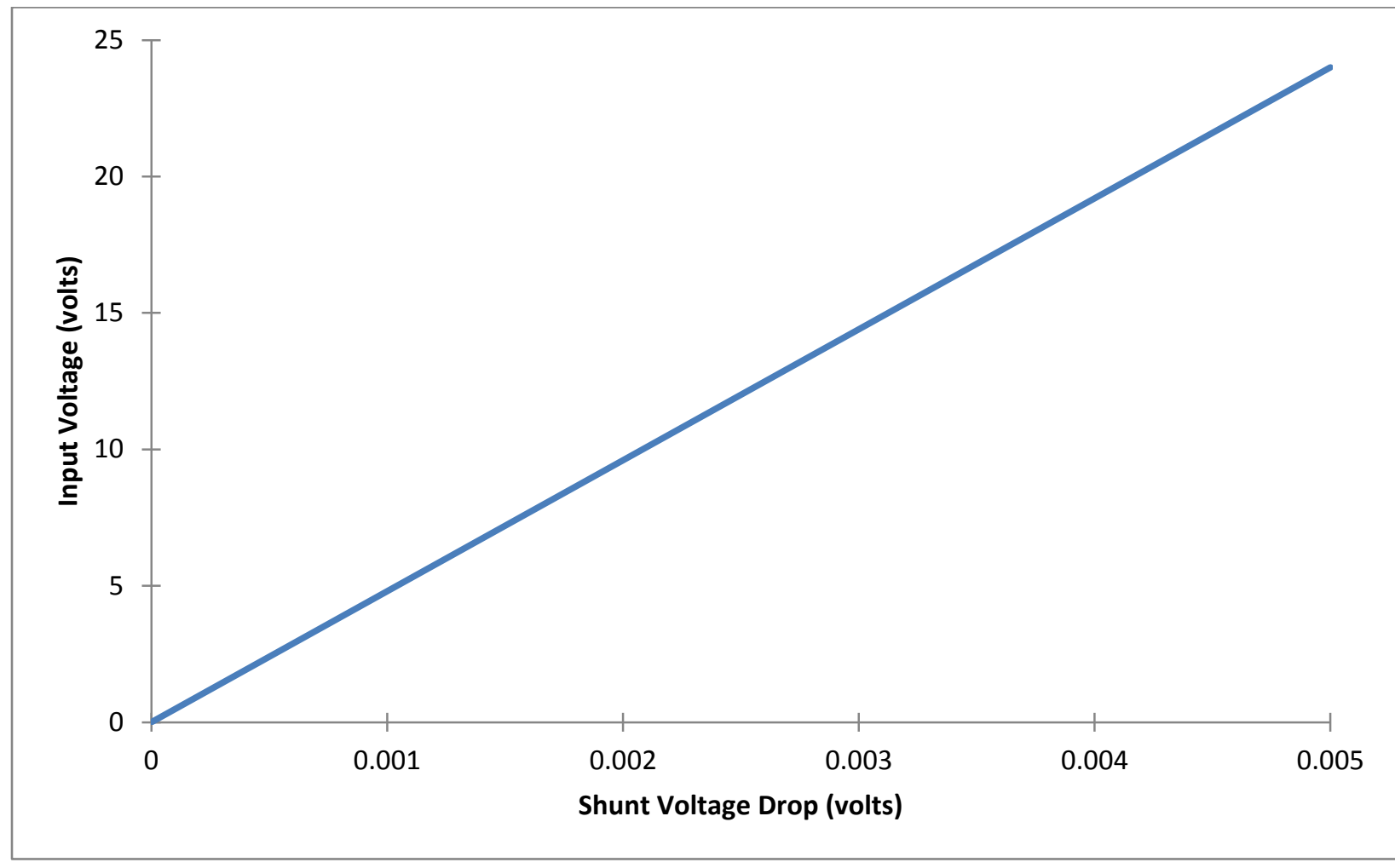

Figure 55. Relationship of Input Voltage to Shunt Voltage Reading

Using a known resistance in the shunt and the measured $D C$ voltage drop, a known value of motor current was extrapolated and used to gather power input data while the motor was in use. The final experimental reading was taken with the help of two ultrasonic anemometer probes inserted in the flow field to monitor the wind speed of the tunnel (acting as forward velocity components and plenum pressurization forces). The anemometers were mounted in two locations, one forward of the propeller plane and one aft. The original calibration was done by 
traversing the wind tunnel with one of the anemometer probes while varying the tunnel air velocity. The results of this preliminary study were then compared to computational results in order to characterize the tunnel and to obtain a predictable forward velocity reading during testing. The results of this characterization are given in later sections.

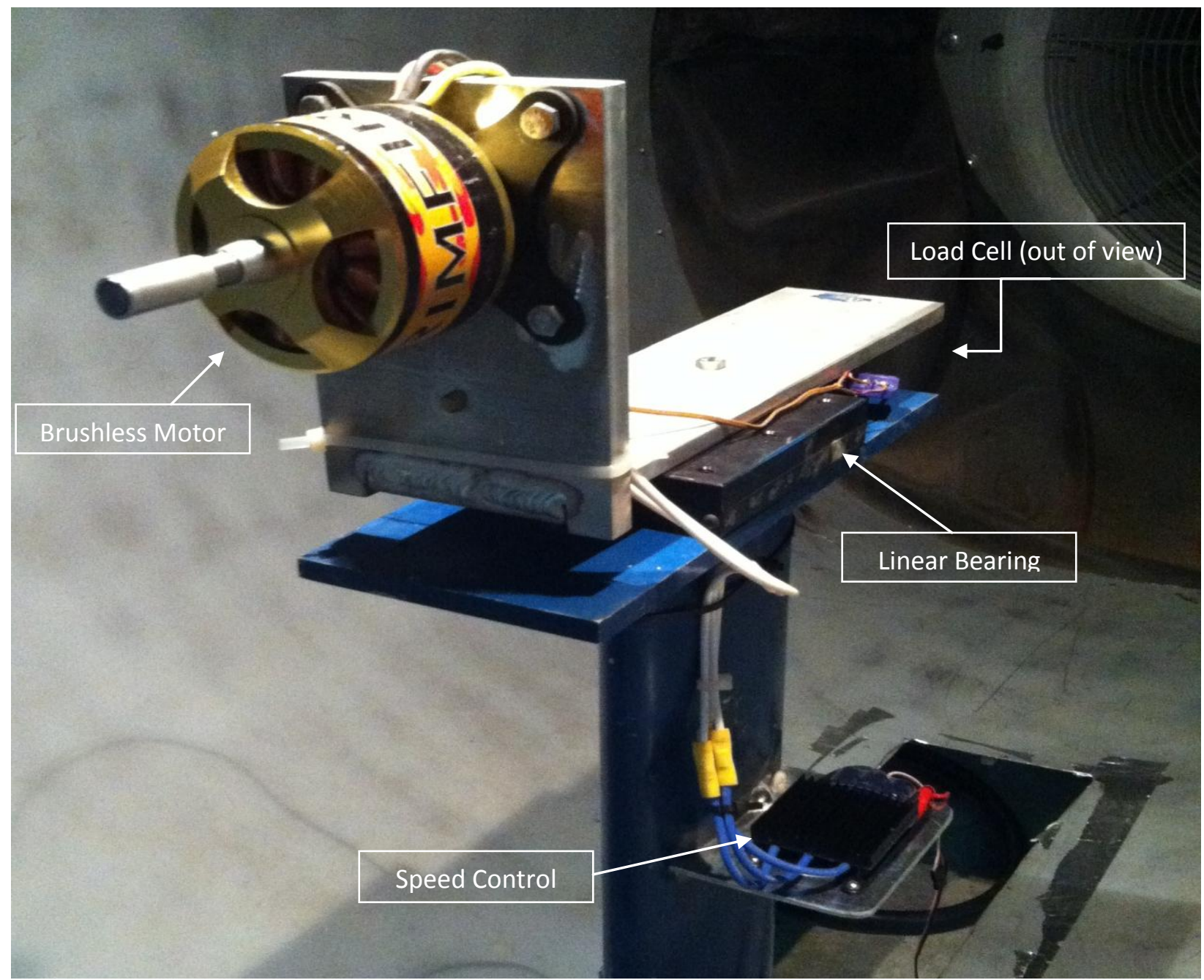

Figure 56. Test Stand and Motor Mounted in Wind Tunnel

\section{Load Cell Calibration}

A load cell calibration stand was constructed outside of the wind tunnel environment in order to accurately test and reexamine the output of the strain gauge used in the experimental process. In conjunction with the linear bearing system on the model stand, a load cell was 
mounted in line with the thrust direction of the propeller axis. Several weighted objects were hung off the test rig attached with a $0.0048 \mathrm{~m}(3 / 16$ inch) steel cable.

When using a linear-type gauge, three testing points are the minimum amount to get a repeatable trend value. For this calibration set, ten different weights ranging from 0 to 231 Newtons (0.0-52.0 pounds) were chosen to load the strain gauge to be positive that the gauge was working properly. The actual values of the weights were also checked and found to be accurate with a digitally calibrated scale. It was necessary to validate the strain gauge used in the experimentation in order to make sure the linearity of the readings remained consistent. As the component was originally used in other experimentations, it was possible that the full Wheatstone Bridge could have been overloaded in the past. If this was the case, the gauge would be shown to be unable to return to normal state when weight was removed during calibration, and thus rendered useless for this experimentation.

Below, in Figure 57, a series of calibration runs were reproduced reading the output voltage directly from the strain gauge for each known weight placed on the testing rig. The applications of the weights were randomly varied over a series of testing scenarios. According to these testing scenarios, the gauge functions with a linear output with an accuracy rating of the measured linear trend of 99.8 percent. Averages over multiple runs were considered and a tenpoint calibration curve is shown in Figure 57. This was then used in future experimental testing scenarios to convert the input read voltage from the strain gauge to a thrust force produced by the propeller. 


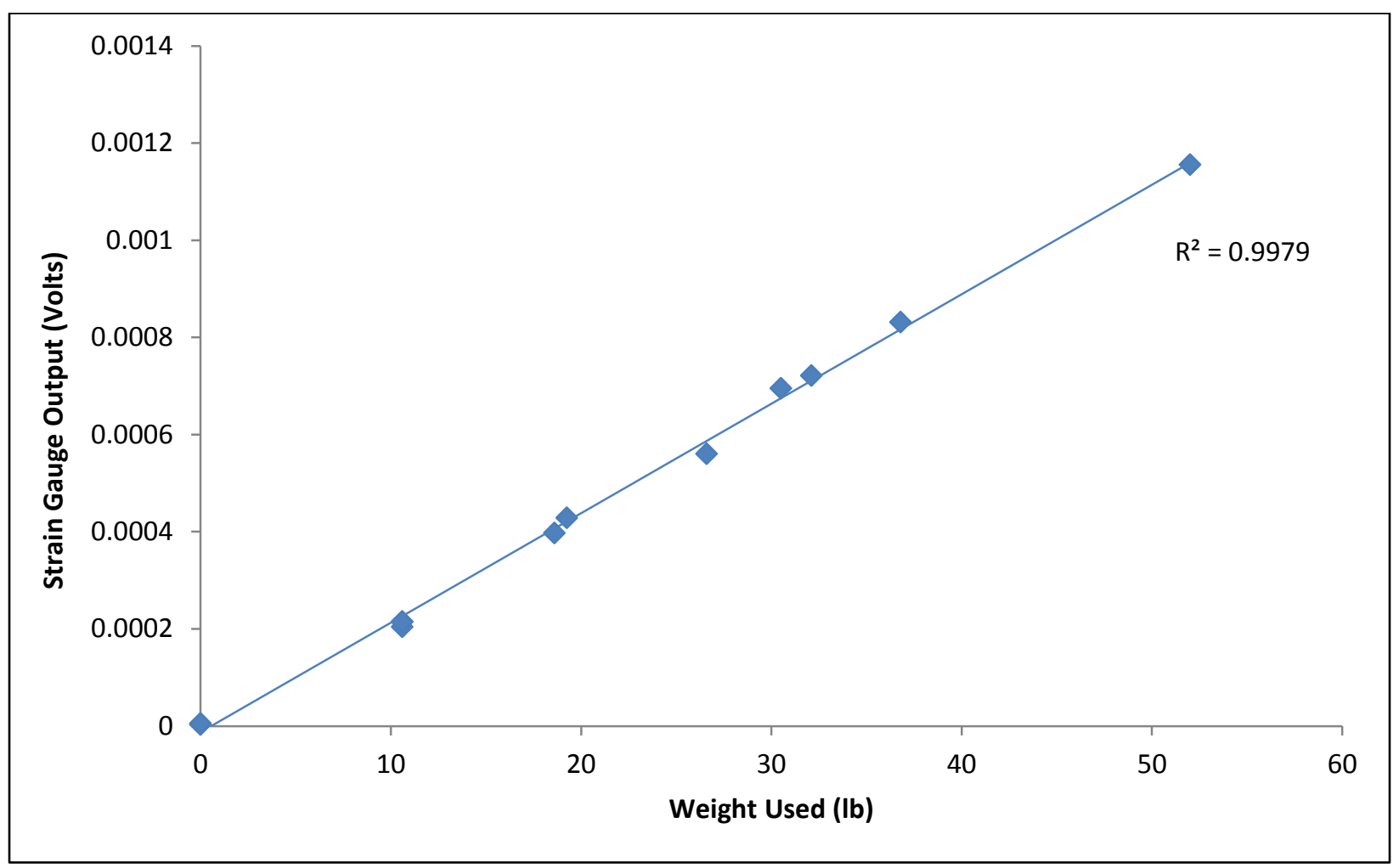

Figure 57. Load Cell Calibration

\section{Electric Motor Connection and Calibration}

The chosen method for driving the propeller during experimentation was a brushless “outrunner" permanent magnet motor called a Rimfire, normally used on model airplanes. The exact model specifications (found in Appendix E - Brushless Motor Specifications) are shown to provide a maximum power rating of 7500 watts, which is roughly equivalent to a power output of 10.0 horse-power (or approximately the same output as a 65.0 cubic centimeter-engine). The rotational speeds of the motor are directly related to the input voltages, referred to as the $k V$ rating. For this brushless motor, the $k V$ rating was given to be 160 (rpm/volt). With the maximum input voltage rated at 55.5 volts, the motor had a maximum rotational speed of 8880 rpm. This output was also dependent on the electronic speed controller (ESC) and the ability it had to cycle power from the source to the motor inputs. Figure 58 shows the unladed motor (no attached propeller) rotational speed prediction based on input control from the servo dial. 


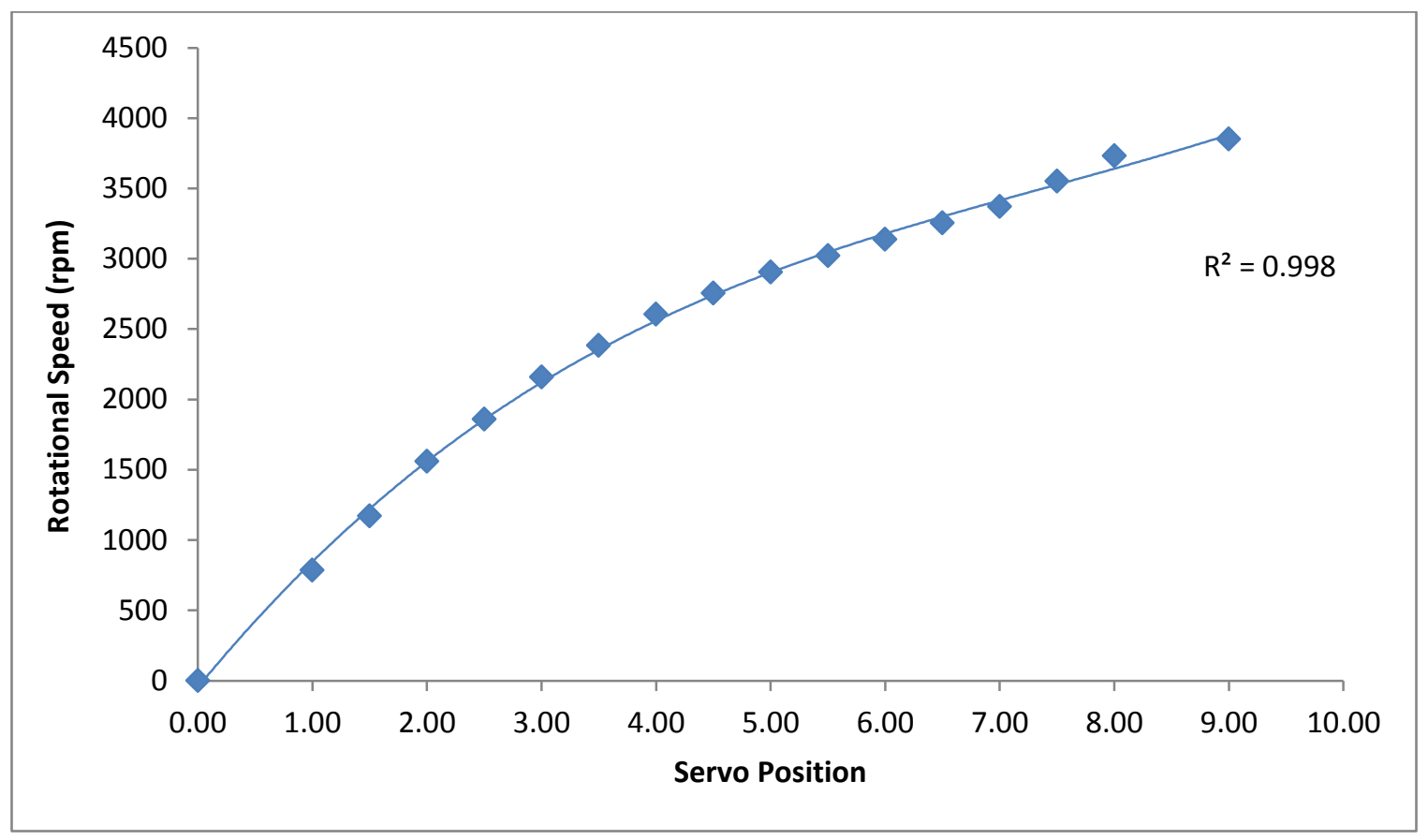

Figure 58 Unloaded Electric Motor Calibration

To control the inputs of the motor at several different rotational speeds and conditions, an electronic speed controller (see Appendix F - Electronic Speed Controller Specifications), shown below in Figure 59, was chosen to wire in line with the motor and power supply. The ESC needed to be able to handle the maximum supply current to the motor (in this case, maximum of 150 amperes instantaneous burst).

The ESC was mounted to the test rig in the downstream of the propeller in the tunnel flow field in order to help provide a cooling gradient and prevent the motor from overheating and burning the coil insulation during long experimental runs. The ESC was mounted on a heat sync, as shown in Figure 59, also to protect the electronics from overheating. Specifications and wiring diagrams can be found for reference in Appendix F - Electronic Speed Controller Specifications.

The second job of the ESC was to take the direct current voltage from the battery bank and convert it to alternating three phase current for use in the brushless motor. In Figure 59, it 
can be seen that the two leads entering the ESC are converted to three leads coming out of the ESC and running into the motor.

An issue that was found after installation was that the ESC is programmed according to lithium-polymer $(\mathrm{Li}-\mathrm{Po})$ battery cells it is attached to, and is "smart" enough to know how many it is connected to at any one time. As the experimental power source for this experiment was Nickel-Metal Hydride (NI-MH) car batteries, the ESC read the input accordingly and would show an over-voltage with any input above two total batteries, or 24.0 volts. This became the maximum input voltage value that the motor would be able to accept, roughly equivalent to 4000 rpm, under unloaded conditions (no propeller attached). This required several extra steps in planning experimental runs in order to analyze a full spectrum of advance ratios of the two experimental propellers. By varying the forward velocity from $0.0-10.0 \mathrm{mps}$ and varying the rotational speeds of the propeller throughout the maximum input $(0-4000 \mathrm{rpm})$ to the motor, a broad range of experimental runs were conducted over a vast array of advance ratios $(J)$, in order to most accurately study the effect of circulation control on the propeller. Specifically these experiments looked at low values of advance ratios, 0.0 to 0.5 . 


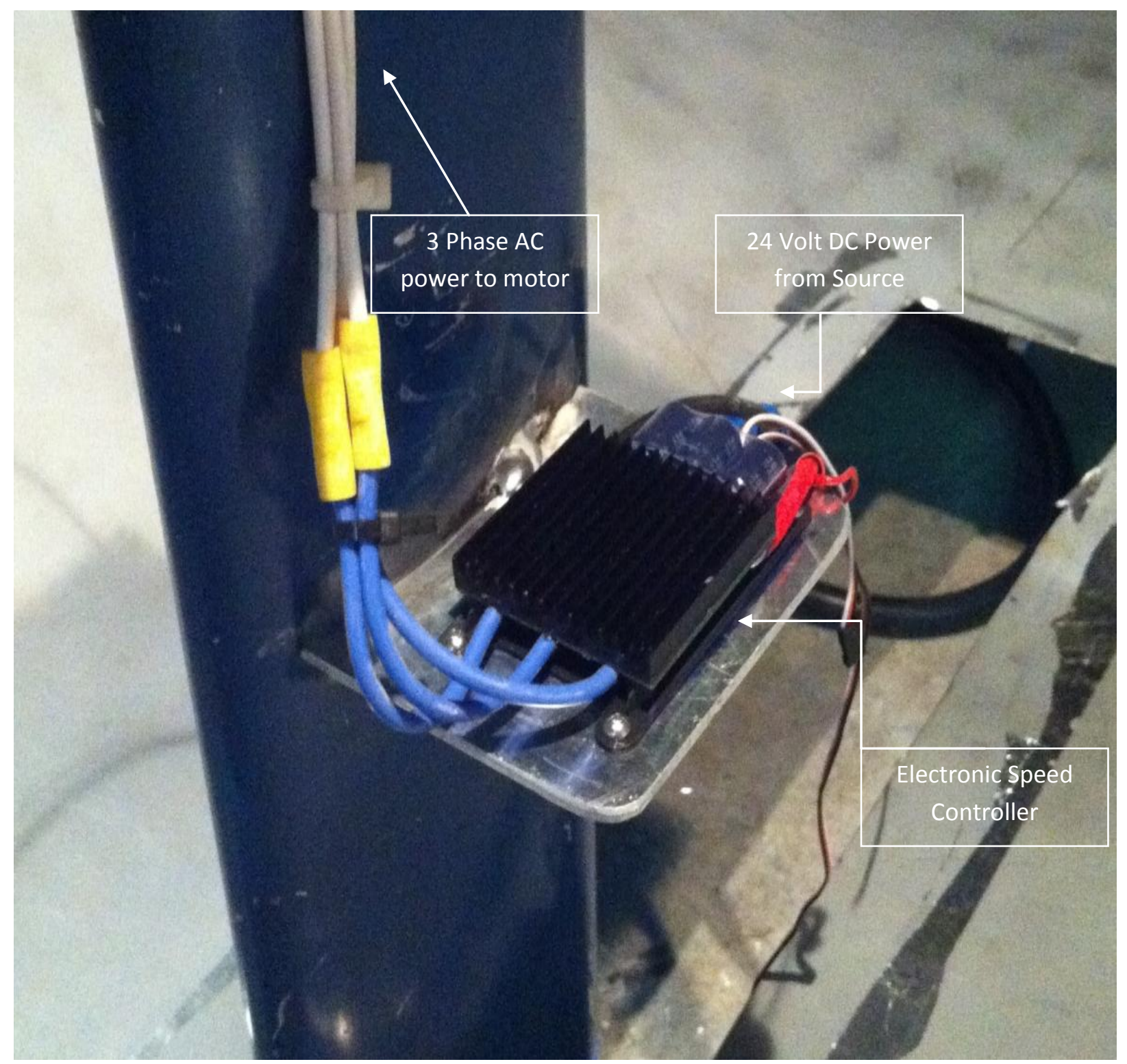

Figure 59. ESC Mounted on Experimental Test Stand

\section{Wind Tunnel Characterization}

Wind tunnel characterization is an aerodynamic way of modeling and predicting how an experiment is going to behave according to aerodynamic laws. In order to achieve reliable repeatable results from a wind tunnel environment the airflow needs to be measured and analyzed from several locations both upstream and downstream of the model.

It also helps in developing an experiment to examine the flow while the model and testing apparatus is not inserted into the tunnel. While testing the tunnel without the model 
inserted, it is important to note that the resulting flow is merely an ideal situation and can theoretically only be useful when comparing experimental results to analytical and/or computational results. Figure 60, shows a normal visualization of the transition of flow inlet to fully developed flow over the entrance of a tunnel environment. It is important to note the no-slip condition present at the tunnel walls, as the fluid adheres to viscous fluid flow mechanics.

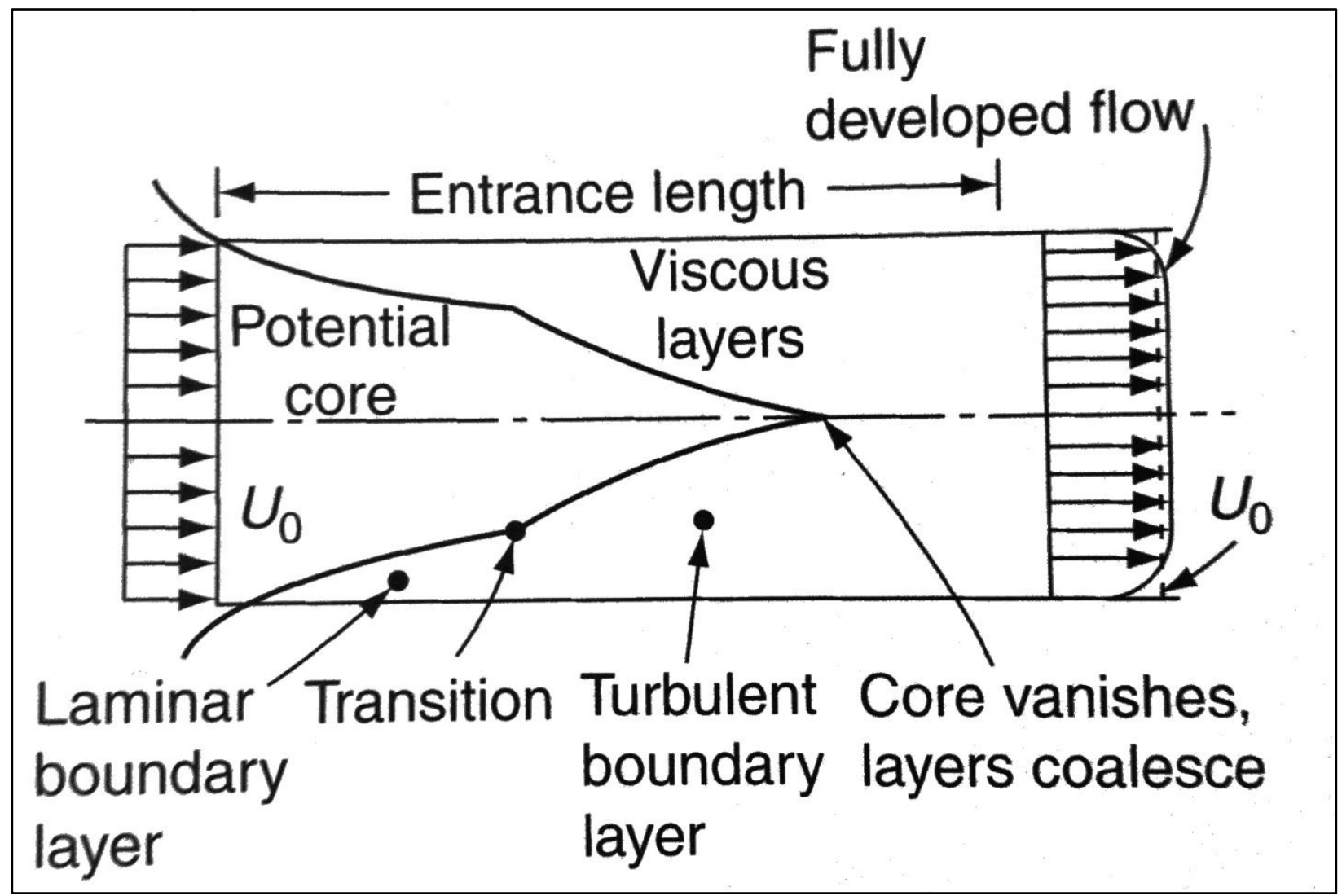

Figure 60. Normal Flow inside the entrance of a Tunnel (White, 2006)

Tunnel head velocities were measured in the $W V U$ wind tunnel with the aid of an ultrasonic anemometer positioned at several radial locations across the cross-section of the tunnel diameter, as well as at several tunnel lengths downstream of the entrance. The drive fan frequency control was used to monitor the tunnel outlet settings while the measurements were being recorded. Frequencies were chosen between 0-60 Hz, in order to obtain a full spectrum of tunnel velocities that the fan could provide. Below, in Figure 61, another visual representation of 
the fluid flow into a tunnel environment is shown, this time with the help of a stream of bubbles inserted into the flow field. The flow visualization accurately portrays the transition of the inlet velocity through the tunnel to a fully developed flow condition at the exit.

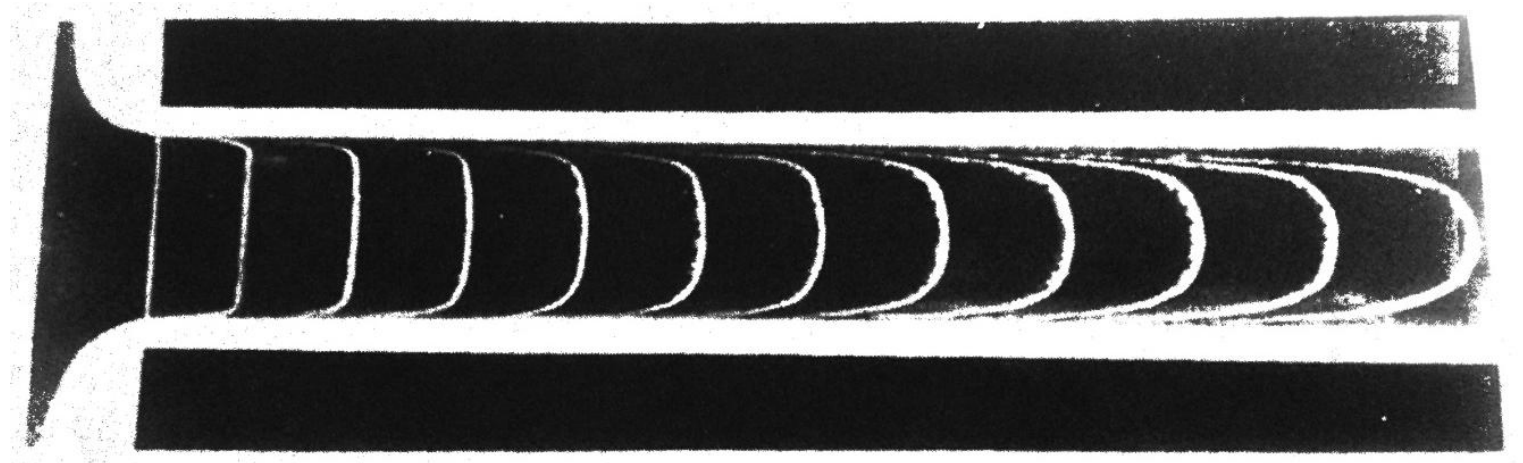

Figure 61. Bubble Simulation of Velocity Cross-Section Inside a Tunnel (White, 2006)

The theory of viscous fluid flow was used in determining the flow conditions of the tunnel and the placement of the model in the downstream flow. It was necessary to find where the fully developed flow characteristics were present in the tunnel environment, and to use this location as the inlet plane for the experimental propeller apparatus. Analyzing the experimental model in the fully-developed flow conditions of the tunnel, allowed for the highest amount of predictability and experimental accuracy while testing.

Measurements were taken in three dimensions, along the length of the tunnel (at stations measured at $0.152,0.664$, and 1.118 meters from the inlet of the tunnel), and were also taken in the cross sectional dimensions of the tunnel (at $0.203,0.483,0.762$, and 1.041 meters across the tunnel and $0.381,0.737,1.143$ meters from the bottom of the tunnel). These values were converted into radial locations in polar coordinates and used along with the length characteristics to design a non-dimensional value of position within the tunnel. Measurements were also taken over numerous tunnel heads, controlled by the motor frequency drive $(4.1,29.0,43.0$, and 60.0 $\mathrm{Hz}$ ) which theoretically corresponded to an inlet air velocity of $0.5,3.0,7.0$ and $10.0 \mathrm{~m} / \mathrm{s}$. 
In the following images, Figure 62, Figure 63, and Figure 64 velocity measurements were taken with an ultrasonic anemometer at various planes across the experimental tunnel cross section. A "tunnel location" variable $(L / r)$ was extrapolated based on the velocity measurements position within the tunnel. Theoretically, the larger the $L / r$ number, the closer to the propeller plane the measurement was taken. It is shown that as the value of $L / r$ increases to above 4.00 , the flow of the tunnel begins to even out and settle, providing a repeatable test section tunnel velocity based on input frequency from the motor drive. The velocities attainable for testing range between $0.25 \mathrm{~m} / \mathrm{s}$ to $10 \mathrm{~m} / \mathrm{s}$ a the maximum fan input values. The repeatable nature of the tunnel velocity close to the propeller plane suggests that the flow has fully developed and can be used to obtain reliable experimentation results.

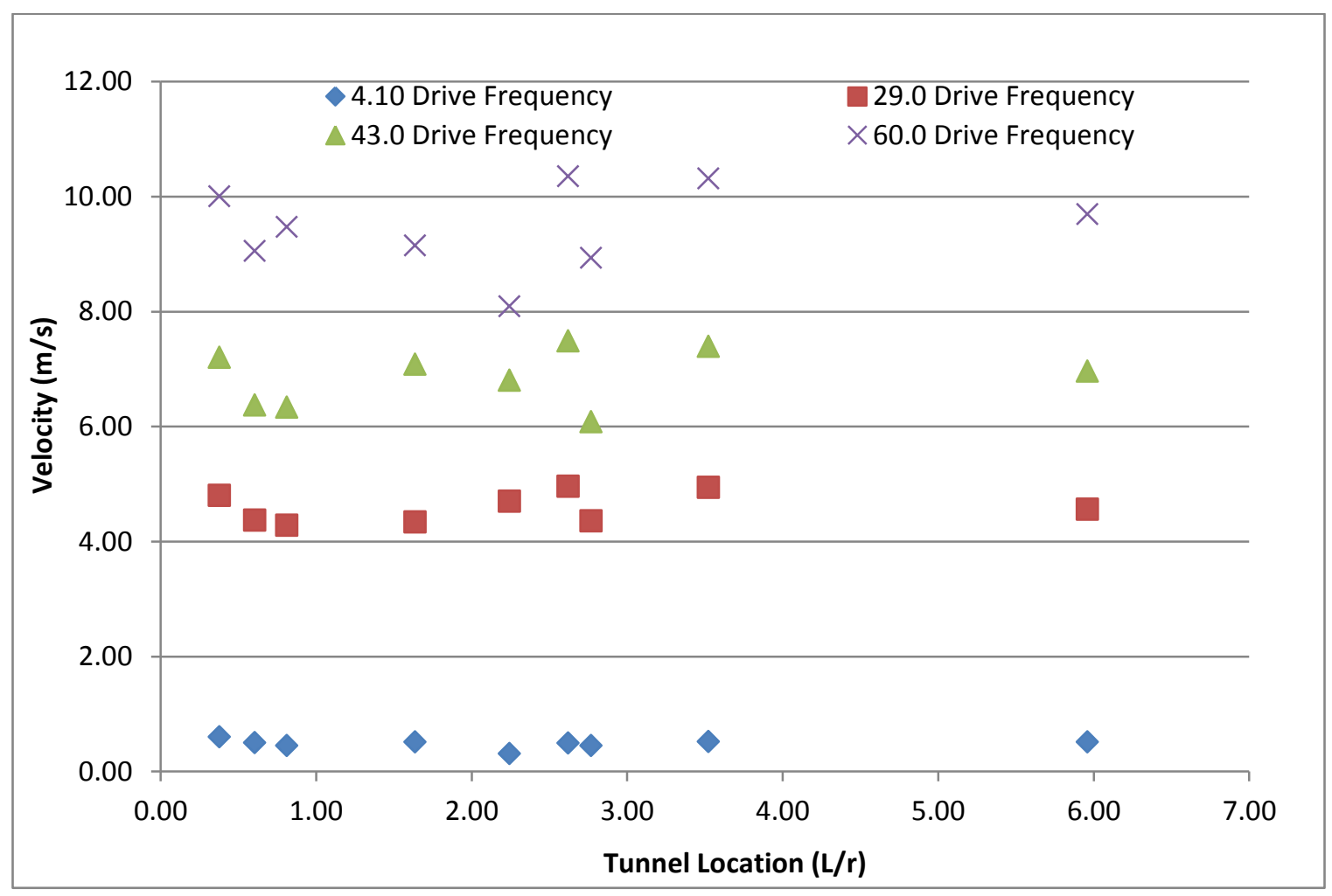

Figure 62. Velocity Measurements Taken at Centerline ( 0.737 meters from Bottom) 


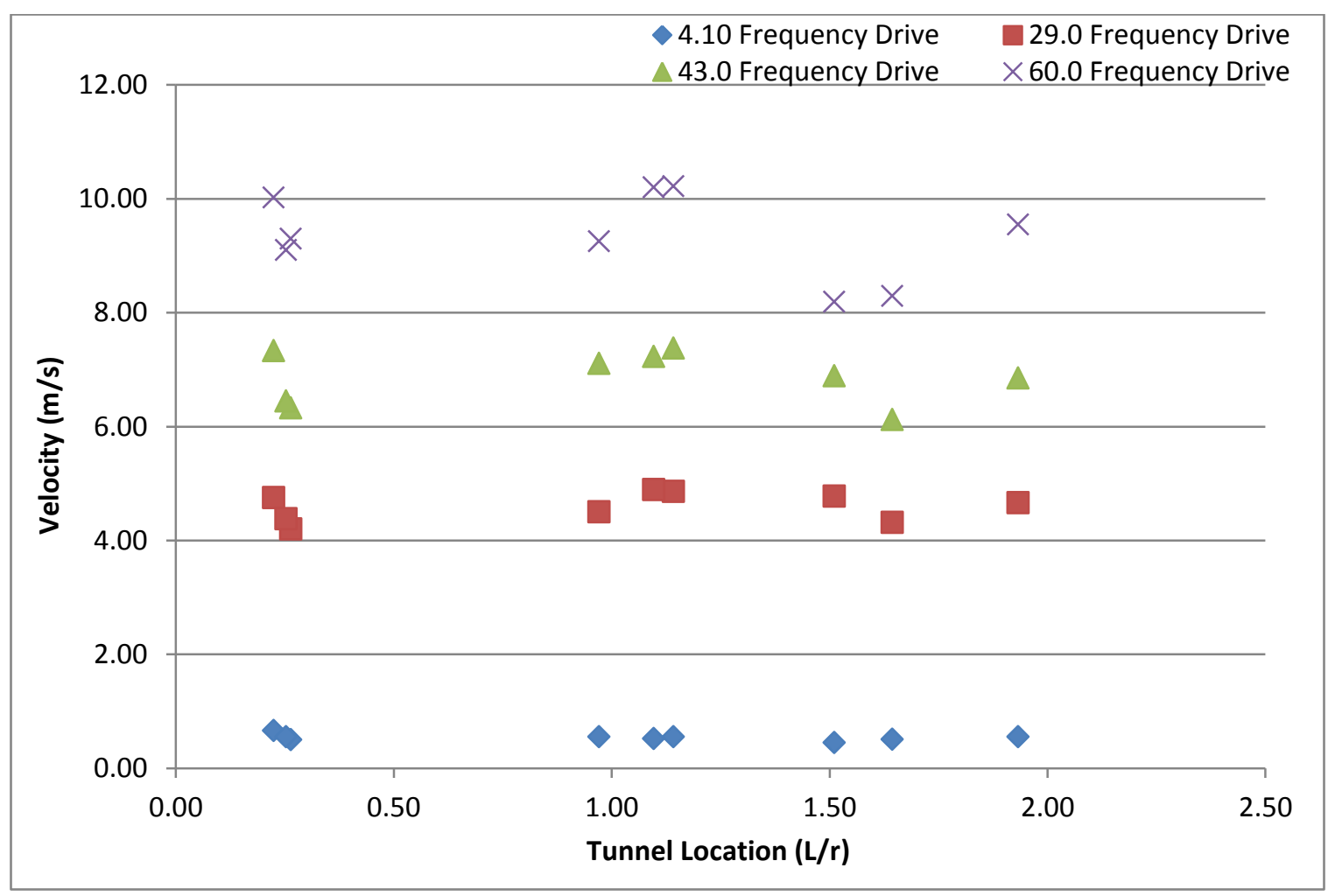

Figure 63. Velocity Measurements Taken Above Centerline ( 1.143 meters from Bottom)

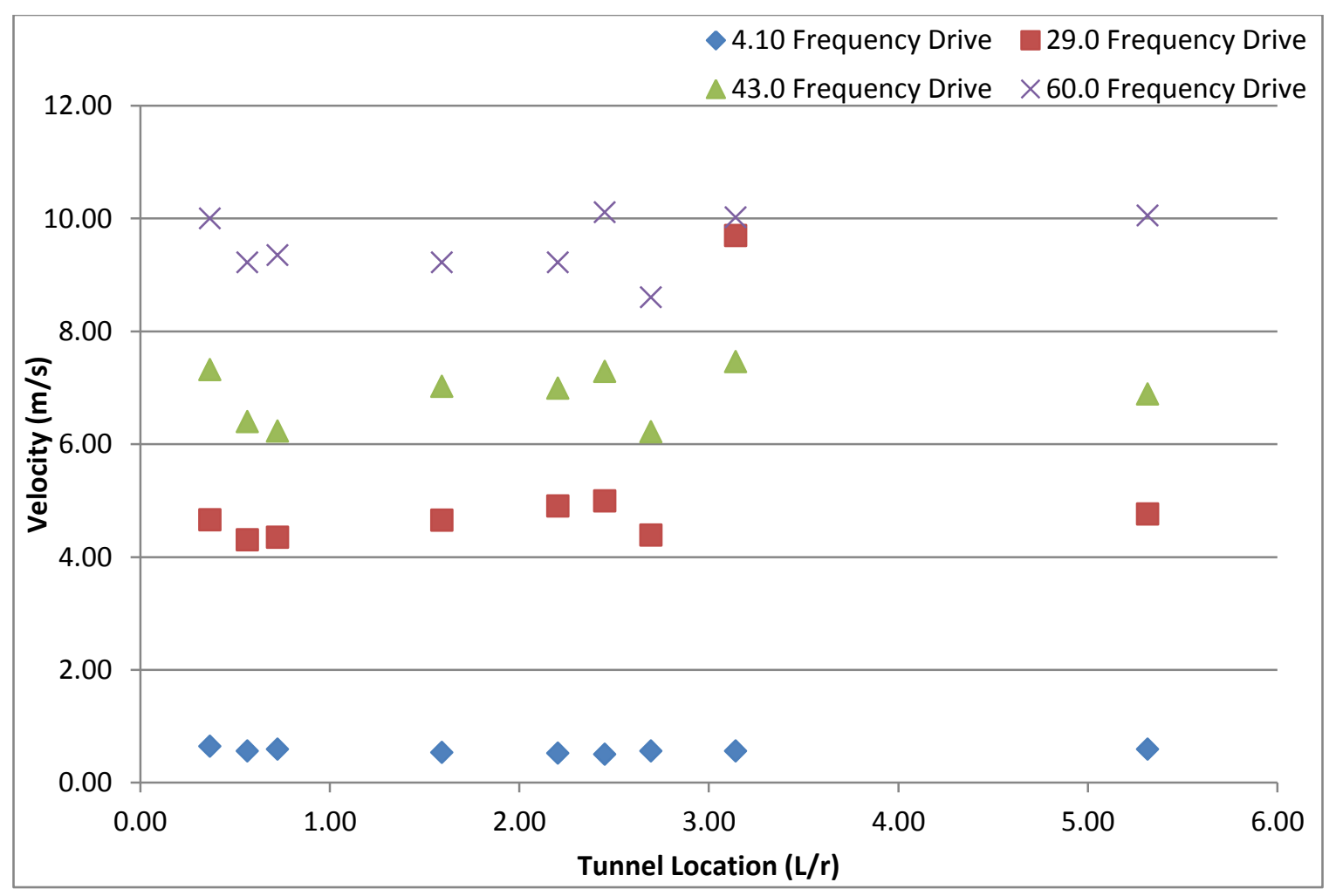

Figure 64. Velocity Measurements Taken Below Centerline ( 0.381 meters from Bottom) 


\section{WPAFB/AFRL Experimental Apparatus}

A secondary set of experimental trials were completed to analyze the effect of the propeller ducting system at higher forward velocities, as well as at angles-of-attack with respect to the free-stream velocity. These tests were conducted at the Wright-Patterson Air Force Base $(W P A F B)$ - Air Force Research Laboratory $(A F R L)$ Vertical Wind Tunnel facility in Dayton, $\mathrm{OH}$. The tunnel is a building itself, with a large rotating fan in the ceiling pulling air through some veins and up into a test section, with a diameter of 3.60 meters (12.0 feet). The test section, model mounting sting, and force balance are all shown in Figure 65.

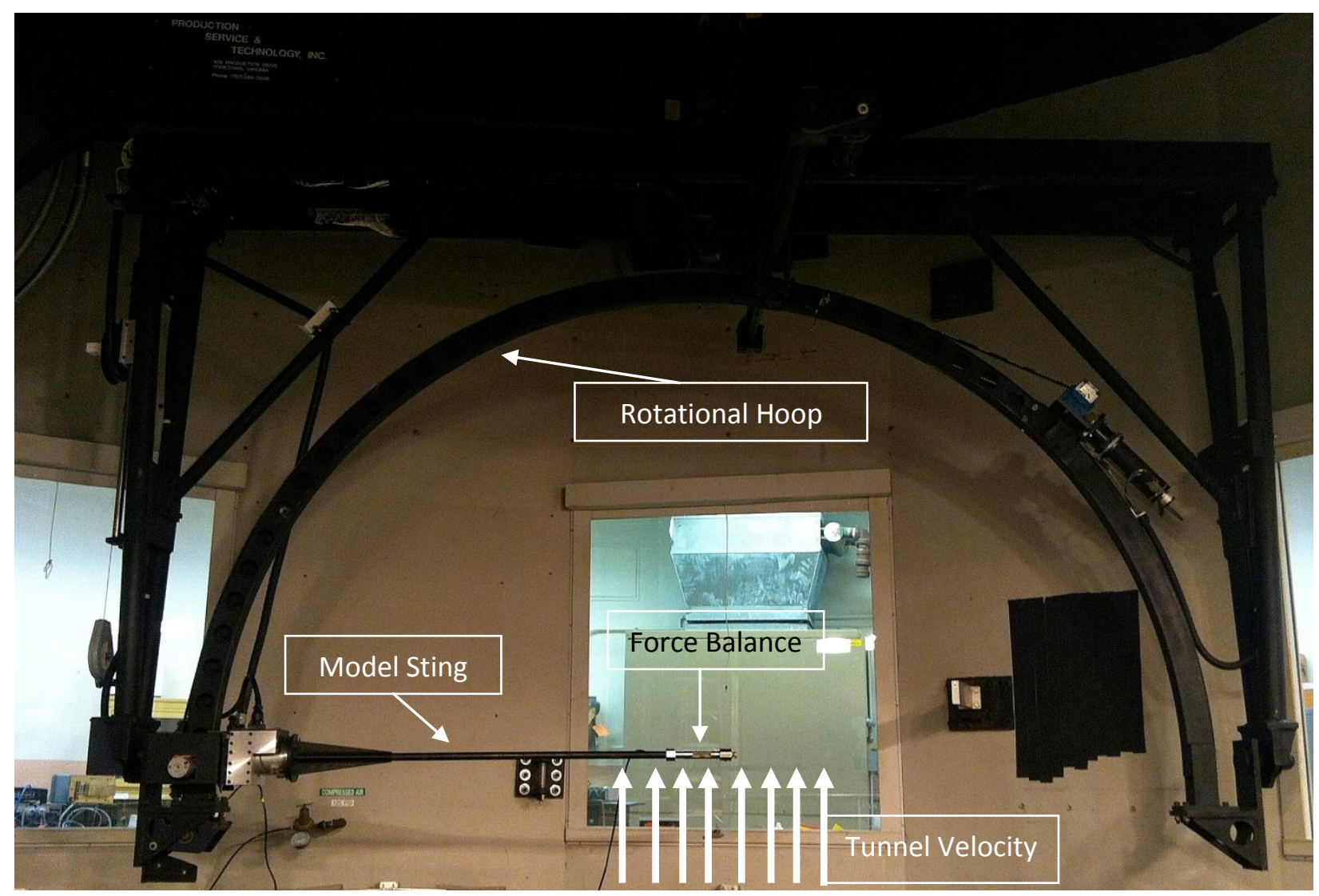

Figure 65. Test Apparatus for Mounting Propeller

The air then circulates through the building along the outside walls of the test section funneling back up through the floor and back through the test area. The maximum forward 
speed achievable for testing in this tunnel was 46.1 meters per second (103.0 miles per hour), giving the ability to experiment at higher advance ratios, 0.60 to 1.20 without having to change the electrical connections of the motor to accommodate voltage input for larger rotational speeds. This prevented damage to the propeller model from very high rotational speeds. Along with increased speed characteristics of the propeller, the tunnel also allowed for the entire disk plane to be slanted, as an angle-of-attack, into the flow at a known value, simulating a dive or climb type of maneuver (see Figure 66, below).

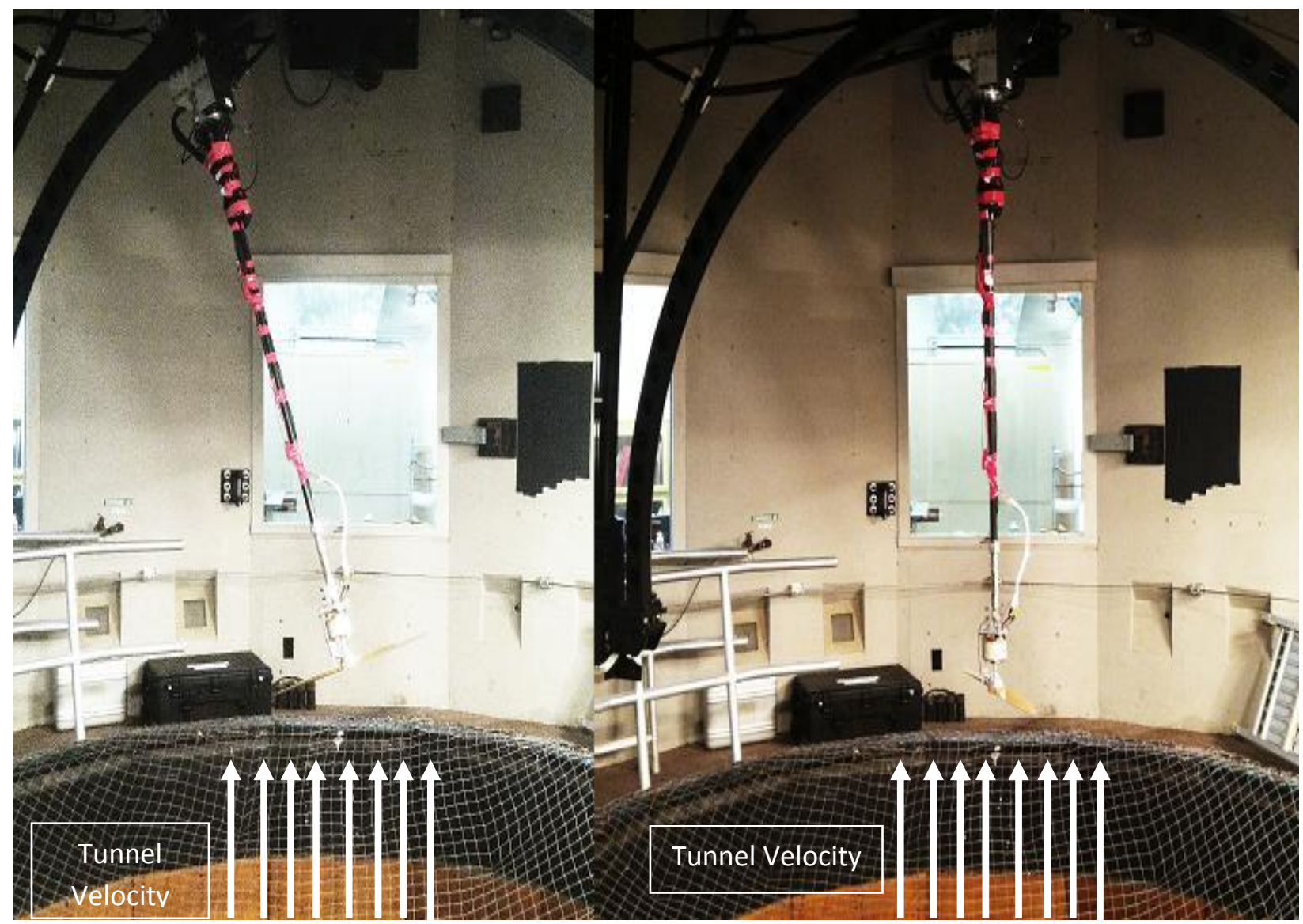

Figure 66. Change in Angle-of-Attack in WPAFB Wind Tunnel

The same motor and ESC from the $W V U$ experimentations were used for the testing scenarios at WPAFB/AFRL and were monitored through a similar data acquisition system. Using a multi-range $D C$ power supply, a signal to the speed controller was used to attain a measurable rotational speed of the motor and propeller. Using the $K v$ rating of the motor, the voltage would 
be extrapolated to an rpm value. The motor was calibrated with a strobe light, similar to the previous experiment, and was also monitored with a Hall Effect sensor mounted directly on the propeller as it was spinning. The Hall Effect sensor set-up comprised of a magnet imbedded into the propeller hub, a sensor relay, and a timer/counter scope. Every time the propeller magnet passed by the sensor relay, one rotation was measured. The counter monitored the amount of rotations the propeller was turning in one minute (output in $\mathrm{Hz}$ ). This three-way verification allowed for precise measurement of rpm at any experimental condition.

Thrust loading was measured directly with a three component force balance mounted directly to the tunnel sting and in line with the propeller, illustrated below in Figure 67. The maximum loading capability for the force balance was 88.9 Newtons (20 pounds) before there was failure in the accuracy of the readings. This was more than sufficient for experimentation in comparison to the maximum static predicted thrust loads of 60.0 Newtons (13 pounds). An overall view of the experimental hardware is shown in Figure 68.

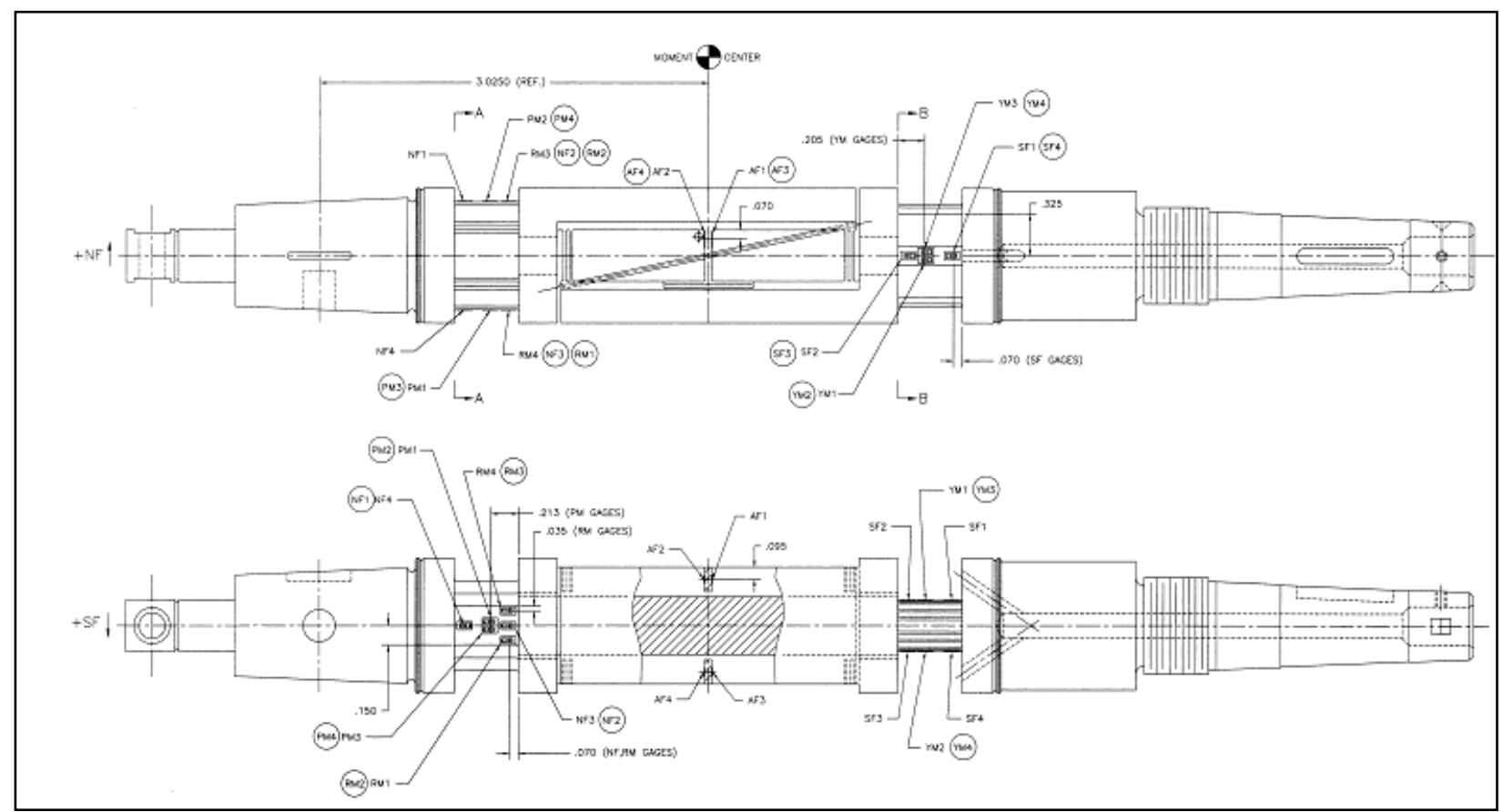

Figure 67. Force Balance Engineering Drawings 


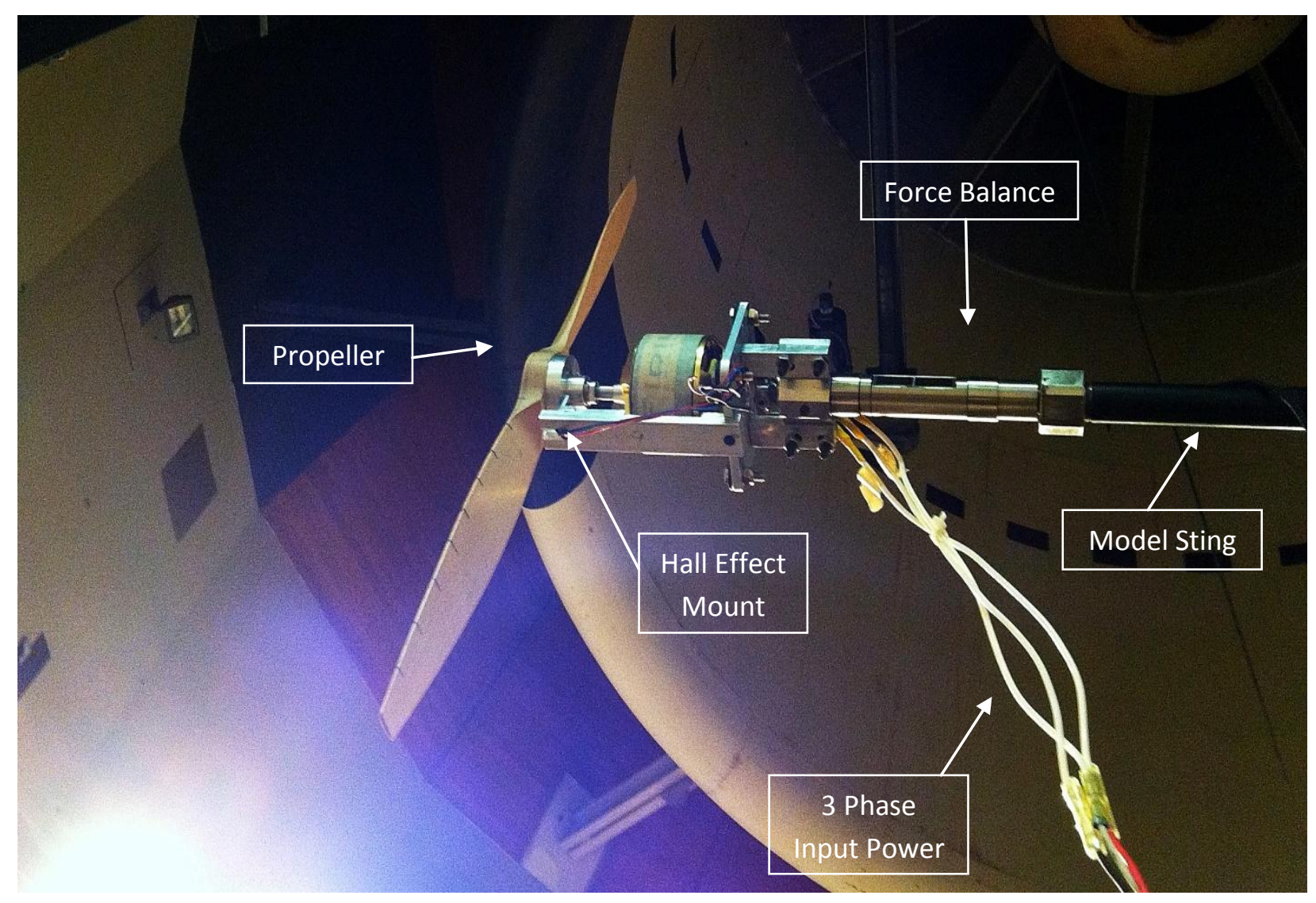

\section{Figure 68. Propeller, Motor, and Force Balance Mounted in WPAFB Wind Tunnel}

The input torque of the motor was again monitored through the input voltage and current of the power supply, shown in Figure 69. With a known input value for the voltage and electrical current loading on the motor, a torque input was able to be back-calculated based on the rotational speed measured on the motor. This rotational speed was measured with the Hall Effect sensor mounted to the propeller hub, able to rotate with the entire assembly. The other half of this magnetic sensor was statically mounted directly to the motor. Similarly to previous experimentation, the thrust and torque forces were then related to non-dimensional coefficients and compiled with the previous data taken on the $W V U$ campus.

A second method of measurement was used to tabulate values of torque for this particular experiment. With the addition of a six component force balance to the system, the ability to directly measure the rolling moment during testing gave an accurate measurement of the torque 
input needed to turn the propeller. Using these two methods allowed for a more accurate dual faceted measurement during experimentation. This method, showed a direct correlation of results of the two measurement systems, and thus essentially proved that measuring the input current and voltage and extrapolating a rotational speed of the motor to measure the input torque, was accurate enough for experimental testing, and much less expensive than purchasing a three component force balance.

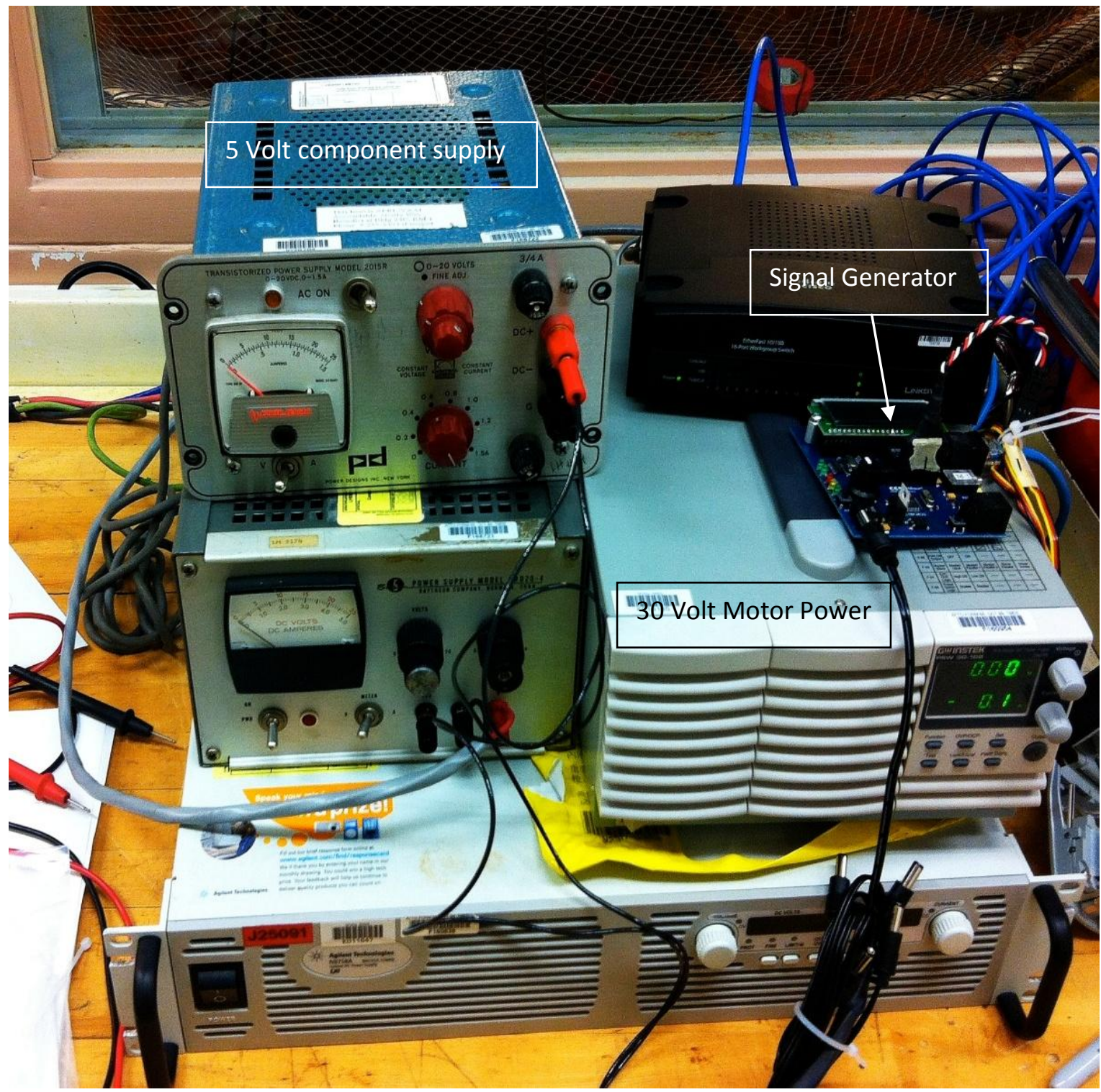

Figure 69. Power Supply System at WPAFB 


\section{WPAFB Wind Tunnel Characterization}

There were several methods available to calibrate the wind tunnel at WPAFB based on the available tools already employed on-site. Some of this information was gathered prior to this experiment and used as a comparison between the overall test cases. The data was gathered in relation to the tunnel fan rpm because the data acquisition software was already written to easily change fan speed, but the gathered data was also used to update the software in the future to be able to change tunnel freestream velocity directly. Below, in Figure 70, several different test cases are shown from gathering tunnel velocities using different measurement instruments.

The data collected with an ultrasonic anemometer was used as the historical benchmark. This device is linear by design, and is mounted on the tunnel wall, 0.457 meters ( 1.5 feet $)$ from the tunnel wall. The anemometer is a stationary fixture of the tunnel and is always reading values, even while testing is in progress.

The "Temp Pitot Avg" is a simple pitot probe mounted on a stand. The velocity measurements were taken as the sensor was moved around the test section at a randomly placed points, but always within 0.914 meters ( 3 feet) radially of center. In these experiments, one data set was reduced with the test rig inserted in the flow and a second set of data was taken with the rig removed from the freestream. This provided data on the drag of the tunnel test rig, i.e. difference between green and dashed blue lines in Figure 70.

The "Wall Pitot" is a sensor attached directly to the tunnel wall and similar to the ultrasonic anemometer, is always collecting data. This sensor is mounted 0.229 meters ( 9 inches off the tunnel wall, and is normally used in gathering boundary layer experimental data. This sensor still shows a repeatable velocity contour for values close to the tunnel wall.

The sting mounted pitot probe gave the best reading of velocity that any model would see if directly mounted to the tunnel sting. The resulting data agrees with the previous existing data 
trends, and shows only 1-2 percent difference between the last pitot probe analysis. The "Windtronic Handheld" data seems to show a slightly higher reading than all the other experimental values, however, it was expected to have some human error associated with the final results as it was manually held in the tunnel flow.

The trend of the velocity contours match the other values produced by different hardware measurements. The resulting information taken from this calibration is that the velocities read from the permanently mounted ultrasonic anemometer are closely related to the tunnel velocities experienced by the model in the tunnel freestream flow (within a 5\% difference throughout the full tunnel speed capabilities).

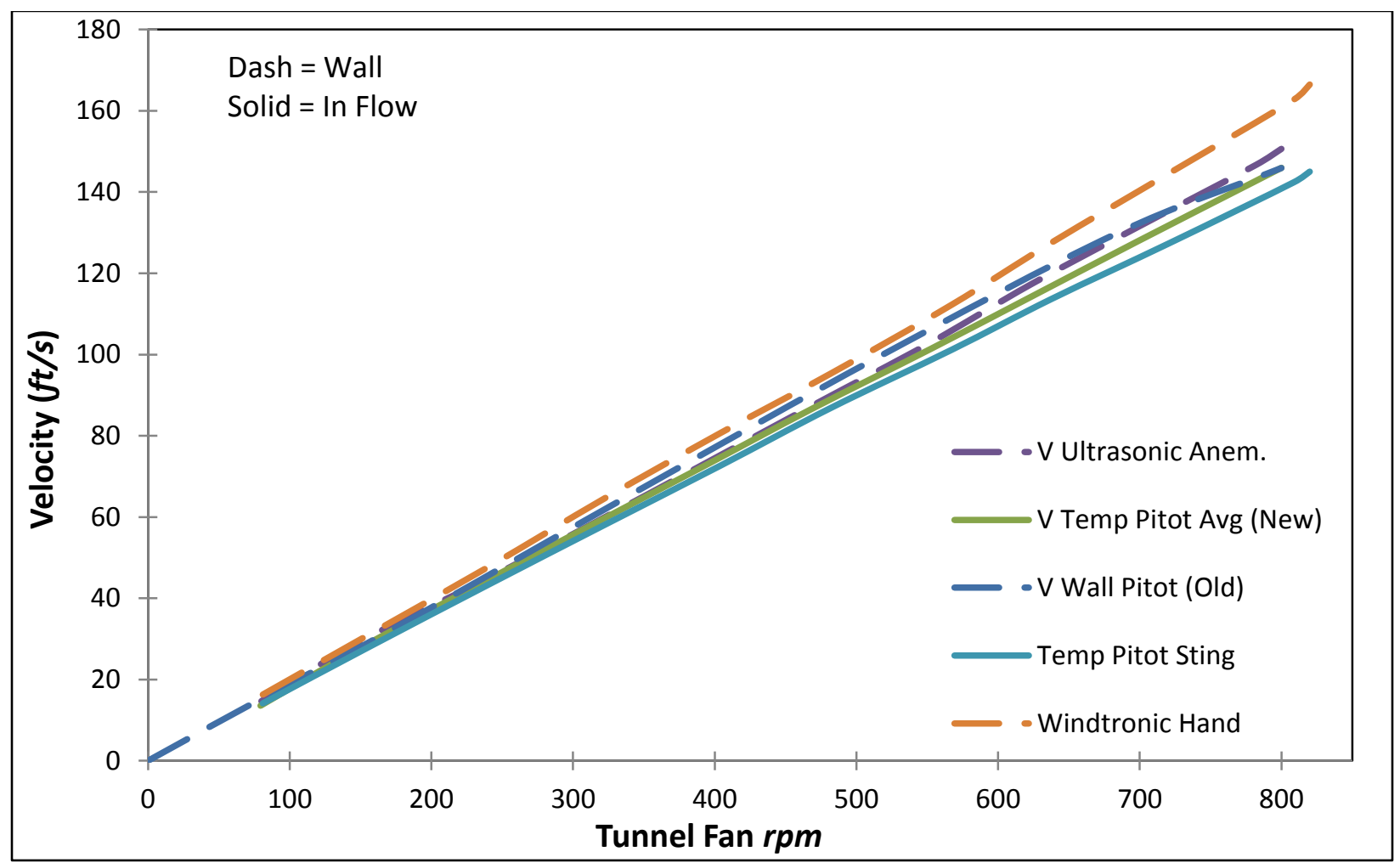

Figure 70. Characteristic Velocity of the Tunnel at WPAFB

\section{Experimental Testing Schedule}

With a finalized optimum model researched from the background studies and preliminary design efforts, it was necessary to run testing scenarios with a physical model in order to test the 
activated conditions and non-activated conditions of the two propeller models. This is an industry standard for any computational research effort as the complete effects of a new design cannot always be measured and anticipated using computer software analyses. This computational modeling can be useful in future research endeavors for varying the geometry of the propeller inputs and completing fast aerodynamic analyses and can also save time and monetary expenses but only if a generalized computational model and experimental case have agreement in the end. Using the results gathered from the preliminary propeller sensitivity analysis in the sensitivity analysis, a model was created, and tested.

Constructing a viable testing scenario for the propeller in order to accurately estimate the effects of the circulation control plenum and exit slot geometry was an integral part of the experimental process. The ideal situation is to take the smallest amount of data necessary and still be able to extrapolate accurate results. The idea being that the time spent on experimenting and the hardware necessary for the test cases should be minimized, but checked against computational results later in the process.

For this experiment, the variables that were being measured were tunnel head $\left(V_{\infty}\right)$, thrust force $(T)$, and input torque $(Q)$ on the motor. As discussed earlier, these variables were measured as a series of voltages from several different data acquisition devices and computed for both the regular propeller and the $C C$-enhanced propeller. In order to accurately measure the response of the $C C$-augmented propeller, the following data runs were studied (as shown in Table 2). The main variance in the testing schedule was the tunnel head velocity (controlled by electronic motor drive) and the rotational speed of the propeller (controlled via human interface input through a servo tester dial - which regulated the input current to the ESC) 
Table 2. Testing Schedule and Experimental Data Runs

\begin{tabular}{|c|c|c|c|c|c|c|c|c|c|c|c|c|c|c|}
\hline \multirow[t]{2}{*}{$\begin{array}{c}\text { Tunnel } \\
\text { Velocity } \\
(\mathrm{m} / \mathrm{s})\end{array}$} & \multicolumn{2}{|c|}{$\begin{array}{c}0.0 \text { (Static } \\
\text { Thrust) }\end{array}$} & \multicolumn{2}{|c|}{0.5} & \multicolumn{2}{|c|}{2.5} & \multicolumn{2}{|c|}{3.5} & \multicolumn{2}{|c|}{5.5} & \multicolumn{2}{|c|}{8.5} & \multicolumn{2}{|c|}{10} \\
\hline & 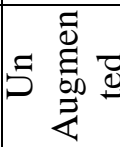 & 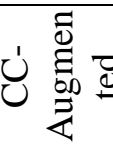 & 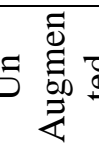 & 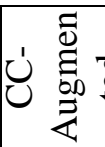 & 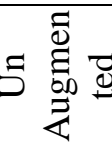 & 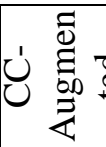 & 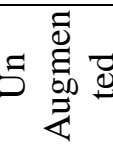 & 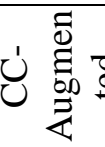 & 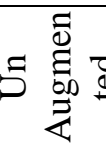 & 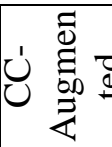 & 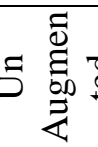 & 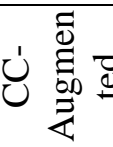 & 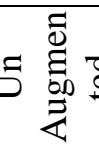 & U \\
\hline \multirow{16}{*}{ 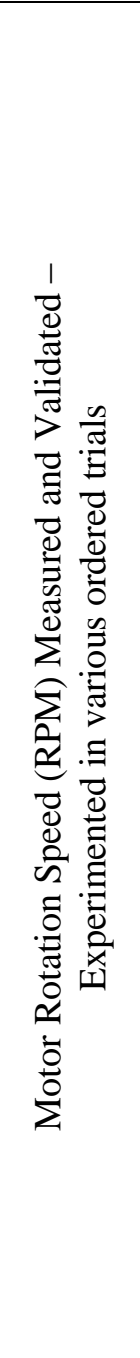 } & 784 & 784 & 784 & 784 & 784 & 784 & 784 & 784 & 784 & 784 & 784 & 784 & 784 & 784 \\
\hline & 1172 & 1172 & 1172 & 1172 & 1172 & 1172 & 1172 & 1172 & 1172 & 1172 & 1172 & 1172 & 1172 & 1172 \\
\hline & 1560 & 1560 & 1560 & 1560 & 1560 & 1560 & 1560 & 1560 & 1560 & 1560 & 1560 & 1560 & 1560 & 1560 \\
\hline & 1860 & 1860 & 1860 & 1860 & 1860 & 1860 & 1860 & 1860 & 1860 & 1860 & 1860 & 1860 & 1860 & 1860 \\
\hline & 2159 & 2159 & 2159 & 2159 & 2159 & 2159 & 2159 & 2159 & 2159 & 2159 & 2159 & 2159 & 2159 & 2159 \\
\hline & 2383 & 2383 & 2383 & 2383 & 2383 & 2383 & 2383 & 2383 & 2383 & 2383 & 2383 & 2383 & 2383 & 2383 \\
\hline & 2606 & 2606 & 2606 & 2606 & 2606 & 2606 & 2606 & 2606 & 2606 & 2606 & 2606 & 2606 & 2606 & 2606 \\
\hline & 2755 & 2755 & 2755 & 2755 & 2755 & 2755 & 2755 & 2755 & 2755 & 2755 & 2755 & 2755 & 2755 & 2755 \\
\hline & 2904 & 2904 & 2904 & 2904 & 2904 & 2904 & 2904 & 2904 & 2904 & 2904 & 2904 & 2904 & 2904 & 2904 \\
\hline & 3021 & 3021 & 3021 & 3021 & 3021 & 3021 & 3021 & 3021 & 3021 & 3021 & 3021 & 3021 & 3021 & 3021 \\
\hline & 3138 & 3138 & 3138 & 3138 & 3138 & 3138 & 3138 & 3138 & 3138 & 3138 & 3138 & 3138 & 3138 & 3138 \\
\hline & 3255 & 3255 & 3255 & 3255 & 3255 & 3255 & 3255 & 3255 & 3255 & 3255 & 3255 & 3255 & 3255 & 3255 \\
\hline & 3371 & 3371 & 3371 & 3371 & 3371 & 3371 & 3371 & 3371 & 3371 & 3371 & 3371 & 3371 & 3371 & 3371 \\
\hline & 3552 & 3552 & 3552 & 3552 & 3552 & 3552 & 3552 & 3552 & 3552 & 3552 & 3552 & 3552 & 3552 & 3552 \\
\hline & 3732 & 3732 & 3732 & 3732 & 3732 & 3732 & 3732 & 3732 & 3732 & 3732 & 3732 & 3732 & 3732 & 3732 \\
\hline & 3850 & 3850 & 3850 & 3850 & 3850 & 3850 & 3850 & 3850 & 3850 & 3850 & 3850 & 3850 & 3850 & \begin{tabular}{|l|}
3850 \\
\end{tabular} \\
\hline
\end{tabular}

In order to prevent any "artifacting" in the data acquisition, several precautionary steps were taken during the experimental scenarios. The first step was that the test runs were not conducted in order. This prevented the strain gauge from obtaining a "memory" of where it was in the loading spectrum. Another precaution taken during experimentation was that each of the 
data runs was simulated over the span of 5.0-10.0 seconds of sampling. This allowed for the data acquisition system to take 10.0 samples per second giving 50-100 data samples per trial run.

The computer acquisition program timed each of the test runs on the monitor and the user was responsible for manually turning on and off the logging capabilities. The final precaution taken to ensure accurate experimental data was that each of the trial runs was logged three times in all, again variously ordered throughout the entire testing scenario to avoid "memory" artifacting of the measurement hardware. Each of the measured variables (thrust, input current, and tunnel velocity) were averaged over the time series for each of these trial runs and then were matched with the other two data runs taken with similar conditions and averaged a second time. This allowed for the greatest predicted amount of accuracy in the results of the experimental trials. Each measured variable was recorded over 5 seconds at a $20 \mathrm{~Hz}$ sampling rate, and averaged over a total of three experimental trials. The standard deviation of the measured voltages was found to be a maximum of 0.0000211 volts, corresponding to 4.6 newtons (1.05 pounds) of force.

The wind tunnel used for experimentation at $W V U$, was constructed such that the advance ratios of the propeller ranged from 0.0 to 0.65 . Further experimentation on the circulation control augmented propeller was completed at $W P A F B$ in the vertical tunnel. Similar to the testing scenarios at $W V U$, the experiments at the air force base varied propeller rotational speeds as well as tunnel velocities in order to finish off the spectrum of thrust and input torques required to turn the propellers at various advance ratios.

To accurately test over the entire range of advance ratios and find corresponding efficiency ratios for the augmented propeller, this experimental study tested ranges of advance ratios between 0.3 and 1.1 . The testing schedule used while experimenting in the WPAFB wind tunnel is shown in Table 3, below, and was conducted over a range of tunnel velocities including 
$4.47,8.94$, and 13.412 meters per second $(10,20$, and 30 miles per hour). The output data of this experiment, gave values for the output thrust capabilities of the propeller as well as the input voltage needed to turn the propeller at a specific rotational speed. This input voltage, converted to a power input could then be used to calculate the torque input needed from the motor.

Table 3. Advance Ratio Testing Schedule at WPAFB/AFRL Wind Tunnel

\begin{tabular}{|c|c|c|}
\hline \multicolumn{3}{|c|}{ Propeller } \\
\hline \multicolumn{3}{|c|}{ Tunnel Velocity = 10, 20, 30 miles per hour } \\
\hline Diameter & Rotational Speed & Rotational Speed \\
\hline m & rpm & rps \\
\hline 0.60 & 0 & 0 \\
\hline 0.60 & 600 & 10 \\
\hline 0.60 & 1200 & 20 \\
\hline 0.60 & 1350 & 22.5 \\
\hline 0.60 & 1500 & 25 \\
\hline 0.60 & 1650 & 27.5 \\
\hline 0.60 & 1800 & 30 \\
\hline 0.60 & 1950 & 32.5 \\
\hline 0.60 & 2100 & 35 \\
\hline 0.60 & 2250 & 37.5 \\
\hline 0.60 & 2400 & 40 \\
\hline 0.60 & 2700 & 45 \\
\hline 0.60 & 3000 & 50 \\
\hline 0.60 & 3600 & 60 \\
\hline 0.60 & 3799.98 & 63.333 \\
\hline
\end{tabular}

A secondary set of experimental trials were completed to analyze the effect of the ducting system at higher forward velocities, as well as at angle-of-attack with respect to the free-stream velocity. Along with increased speed characteristics of the propeller, the tunnel also allowed for the entire disk plane to be slanted, at an angle-of-attack, into the free-stream velocity flow at a known value, simulating a fast input dive or climb type of maneuver. This gave experimental results across three different advance ratios for the $C C$ propeller.

The experimental testing schedule is given in Table 4. The purpose of these experiments was only to try to look at preliminary results and see what kinds of overall effects might be anticipated. Clearly, more research would need to be done in order to estimate an overall trend. 
Table 4. Angle-of-Attack Test Schedule at WPAFB/AFRL Vertical Tunnel

\begin{tabular}{|c|c|c|c|c|c|c|c|c|c|}
\hline \multirow{2}{*}{$\begin{array}{l}\text { Alpha } \\
\text { Degree }\end{array}$} & \multirow{2}{*}{\begin{tabular}{|c|} 
Beta \\
Degree \\
\end{tabular}} & \multirow{2}{*}{$\begin{array}{c}\text { Diam. } \\
\mathbf{m}\end{array}$} & \multicolumn{2}{|c|}{ Rotational Speed } & \multicolumn{3}{|c|}{ Tunnel Velocity } & \multirow{2}{*}{$\begin{array}{l}\mathbf{J} \\
-\end{array}$} & \multirow{2}{*}{$\begin{array}{c}\text { Voltage } \\
\text { volts } \\
\end{array}$} \\
\hline & & & rpm & rps & mph & fps & mps & & \\
\hline \multicolumn{10}{|c|}{ Experimental Run \#1 } \\
\hline-30.00 & 0.00 & 0.60 & 3800.00 & 63.33 & 75.00 & 110.00 & 33.53 & 0.88 & 23.03 \\
\hline-25.00 & 0.00 & 0.60 & 3800.00 & 63.33 & 75.00 & 110.00 & 33.53 & 0.88 & 23.03 \\
\hline-20.00 & 0.00 & 0.60 & 3800.00 & 63.33 & 75.00 & 110.00 & 33.53 & 0.88 & 23.03 \\
\hline-15.00 & 0.00 & 0.60 & 3800.00 & 63.33 & 75.00 & 110.00 & 33.53 & 0.88 & 23.03 \\
\hline-10.00 & 0.00 & 0.60 & 3800.00 & 63.33 & 75.00 & 110.00 & 33.53 & 0.88 & 23.03 \\
\hline-5.00 & 0.00 & 0.60 & 3800.00 & 63.33 & 75.00 & 110.00 & 33.53 & 0.88 & 23.03 \\
\hline 0.00 & 0.00 & 0.60 & 3800.00 & 63.33 & 75.00 & 110.00 & 33.53 & 0.88 & 23.03 \\
\hline 5.00 & 0.00 & 0.60 & 3800.00 & 63.33 & 75.00 & 110.00 & 33.53 & 0.88 & 23.03 \\
\hline 10.00 & 0.00 & 0.60 & 3800.00 & 63.33 & 75.00 & 110.00 & 33.53 & 0.88 & 23.03 \\
\hline 15.00 & 0.00 & 0.60 & 3800.00 & 63.33 & 75.00 & 110.00 & 33.53 & 0.88 & 23.03 \\
\hline 20.00 & 0.00 & 0.60 & 3800.00 & 63.33 & 75.00 & 110.00 & 33.53 & 0.88 & 23.03 \\
\hline 25.00 & 0.00 & 0.60 & 3800.00 & 63.33 & 75.00 & 110.00 & 33.53 & 0.88 & 23.03 \\
\hline 30.00 & 0.00 & 0.60 & 3800.00 & 63.33 & 75.00 & 110.00 & 33.53 & 0.88 & 23.03 \\
\hline \multicolumn{10}{|c|}{ Experimental Run \#2 } \\
\hline-30.00 & 0.00 & 0.60 & 3800.00 & 63.33 & 50.00 & 73.33 & 22.35 & 0.59 & 23.03 \\
\hline-25.00 & 0.00 & 0.60 & 3800.00 & 63.33 & 50.00 & 73.33 & 22.35 & 0.59 & 23.03 \\
\hline-20.00 & 0.00 & 0.60 & 3800.00 & 63.33 & 50.00 & 73.33 & 22.35 & 0.59 & 23.03 \\
\hline-15.00 & 0.00 & 0.60 & 3800.00 & 63.33 & 50.00 & 73.33 & 22.35 & 0.59 & 23.03 \\
\hline-10.00 & 0.00 & 0.60 & 3800.00 & 63.33 & 50.00 & 73.33 & 22.35 & 0.59 & 23.03 \\
\hline-5.00 & 0.00 & 0.60 & 3800.00 & 63.33 & 50.00 & 73.33 & 22.35 & 0.59 & 23.03 \\
\hline 0.00 & 0.00 & 0.60 & 3800.00 & 63.33 & 50.00 & 73.33 & 22.35 & 0.59 & 23.03 \\
\hline 5.00 & 0.00 & 0.60 & 3800.00 & 63.33 & 50.00 & 73.33 & 22.35 & 0.59 & 23.03 \\
\hline 10.00 & 0.00 & 0.60 & 3800.00 & 63.33 & 50.00 & 73.33 & 22.35 & 0.59 & 23.03 \\
\hline 15.00 & 0.00 & 0.60 & 3800.00 & 63.33 & 50.00 & 73.33 & 22.35 & 0.59 & 23.03 \\
\hline 20.00 & 0.00 & 0.60 & 3800.00 & 63.33 & 50.00 & 73.33 & 22.35 & 0.59 & 23.03 \\
\hline 25.00 & 0.00 & 0.60 & 3800.00 & 63.33 & 50.00 & 73.33 & 22.35 & 0.59 & 23.03 \\
\hline 30.00 & 0.00 & 0.60 & 3800.00 & 63.33 & 50.00 & 73.33 & 22.35 & 0.59 & 23.03 \\
\hline \multicolumn{10}{|c|}{ Experimental Run \#3 } \\
\hline-30.00 & 0.00 & 0.60 & 3800.00 & 63.33 & 25.00 & 36.67 & 11.18 & 0.29 & 23.03 \\
\hline-25.00 & 0.00 & 0.60 & 3800.00 & 63.33 & 25.00 & 36.67 & 11.18 & 0.29 & 23.03 \\
\hline-20.00 & 0.00 & 0.60 & 3800.00 & 63.33 & 25.00 & 36.67 & 11.18 & 0.29 & 23.03 \\
\hline-15.00 & 0.00 & 0.60 & 3800.00 & 63.33 & 25.00 & 36.67 & 11.18 & 0.29 & 23.03 \\
\hline-10.00 & 0.00 & 0.60 & 3800.00 & 63.33 & 25.00 & 36.67 & 11.18 & 0.29 & 23.03 \\
\hline-5.00 & 0.00 & 0.60 & 3800.00 & 63.33 & 25.00 & 36.67 & 11.18 & 0.29 & 23.03 \\
\hline 0.00 & 0.00 & 0.60 & 3800.00 & 63.33 & 25.00 & 36.67 & 11.18 & 0.29 & 23.03 \\
\hline 5.00 & 0.00 & 0.60 & 3800.00 & 63.33 & 25.00 & 36.67 & 11.18 & 0.29 & 23.03 \\
\hline 10.00 & 0.00 & 0.60 & 3800.00 & 63.33 & 25.00 & 36.67 & 11.18 & 0.29 & 23.03 \\
\hline 15.00 & 0.00 & 0.60 & 3800.00 & 63.33 & 25.00 & 36.67 & 11.18 & 0.29 & 23.03 \\
\hline 20.00 & 0.00 & 0.60 & 3800.00 & 63.33 & 25.00 & 36.67 & 11.18 & 0.29 & 23.03 \\
\hline 25.00 & 0.00 & 0.60 & 3800.00 & 63.33 & 25.00 & 36.67 & 11.18 & 0.29 & 23.03 \\
\hline 30.00 & 0.00 & 0.60 & 3800.00 & 63.33 & 25.00 & 36.67 & 11.18 & 0.29 & 23.03 \\
\hline
\end{tabular}


This data was taken as a preliminary study on the effect of the circulation control slot while maneuvering outside of cruise conditions. The overall experiment was aimed at having a preliminary set of data ranging over the entire rotational values and advance ratios for comparison of performance characteristics between the un-augmented and augmented propellers. For each test case, the propeller was set at a fixed rotational speed and the tunnel velocity was set at a known speed while the angle-of-attack was varied through a spectrum across -30.0 to 30.0 degrees with relation to the free-stream.

As discussed, the thrust was measured with a three axis force balance system which was already mounted in the tunnel and torque was calculated via measured input power, and measured rotational speed. The data runs were sampled at a rate of 200 samples per second, effectively increasing the number of data points at each experimental setting by a factor of 20.0 in comparison to the trials studied at $W V U$. Also present during testing were a series of anemometers and digital pitot probes which were used to monitor the velocity of the tunnel while in operation, similar to the studies conducted at $W V U$. With the data taken and uploaded to a spreadsheet (Microsoft Excel), the next step was to calculate the output variables from the measured data. This can be seen in the results section, later in the text.

\section{Computational Modeling Design and Evaluation}

With results from a historical physical testing scenario (via Zinger, Inc., the propeller manufacturer), it was possible to create a computational model in which certain parameters were changed in order to test a range of geometries, velocities and rotational speeds this propeller could encounter. The first computer simulation needed to verify the historical aerodynamic data available for the selected model propeller. This information can be found below, in Table 5 downloaded from the manufacturer's website. 
The values are based on rotational speeds of the propeller and are also taken at static loading conditions. This fact provides no force data while the propeller is moving at a forward speed. This was used to confirm that the simulation model was calibrated properly.

The circulation controlled propeller was then modeled and simulated using the same testing scenarios in order to predict performance curves. This procedure allowed for complete examination of all parameters, (altitude effects, plenum pressurization, rotational speeds, forward velocity, etc) in order to optimize the entire design without acquiring multiple models for physical examination in a wind tunnel. This computational analysis provided an accurate way to analyze and understand the pressurization effects of the internal ducting of the propeller model while in rotation. With these static values, further computational experimentation was completed studying the forces on the propeller while it was travelling at a forward velocity using a similar computational model.

Table 5. Baseline Propeller Thrust Capabilities- $\left(D=24\right.$ Inch, $\left.P_{*}=10\right)$ (Zinger, Inc., 2010)

\begin{tabular}{|c|c|c|}
\hline $\begin{array}{c}\text { Rotational Speed } \\
\text { (RPM) }\end{array}$ & $\begin{array}{c}\text { Propeller Thrust } \\
\text { (Pounds) }\end{array}$ & $\begin{array}{c}\text { Theory Propeller Thrust } \\
\text { (Newtons) }\end{array}$ \\
\hline 0 & 0 & 0 \\
\hline 500 & 0.28 & 1.26 \\
\hline 1000 & 1.02 & 4.53 \\
\hline 1500 & 2.15 & 9.57 \\
\hline 2000 & 3.66 & 16.27 \\
\hline 2500 & 5.52 & 24.55 \\
\hline 3000 & 7.73 & 34.36 \\
\hline 3500 & 10.27 & 45.66 \\
\hline 4000 & 13.14 & 58.40 \\
\hline 4500 & 16.33 & 72.57 \\
\hline 5000 & 19.83 & 88.13 \\
\hline 5500 & 23.64 & 105.06 \\
\hline 6000 & 27.75 & 123.34 \\
\hline 6500 & 32.16 & 142.95 \\
\hline 7000 & 36.87 & 163.88 \\
\hline 7500 & 41.88 & 186.11 \\
\hline 8000 & 47.17 & 209.63 \\
\hline 9000 & 58.61 & 260.48 \\
\hline
\end{tabular}




\section{Computational Circulation Control Airfoil Profile Analysis}

The augmented analysis was slightly more difficult. Instead of having the ability to look up existing airfoil data and generate Reynolds Number simulations in XFLR-5, or DesignFoil, a computation fluid dynamic model was built to estimate the impact of adding a CC slot to the existing Clark-Y geometry. First, using an electronic model designed in Pro-ENGINEER software, the dimensions of the $C C$ propeller were measured and an airfoil shape was extrapolated. This provided the ability to call up points and profile information of the $C C$ ClarkY airfoil.

Below in, Figure 71, CFD grid spacing is shown around the near field of the airfoil geometry. The rear rounded trailing edge is shown to have a much finer mesh associated, to better estimate the airfoil's interaction with the surrounding fluid. Similarly, in Figure 72, the overall grid mesh is shown for the entire fluid system. Gradually, the grid spacing gets finer and finer as the analysis approaches the surface of the airfoil, allowing for greater expected accuracy in the $C F D$ calculations. A total of 120 nodes were used over the airfoil in the $\mathrm{x}$-direction (chord direction) and a total of 80 nodes were used in the y-direction (normal to the airfoil). This gave a total of 28,264 cells used for analysis (28,454 nodes). 


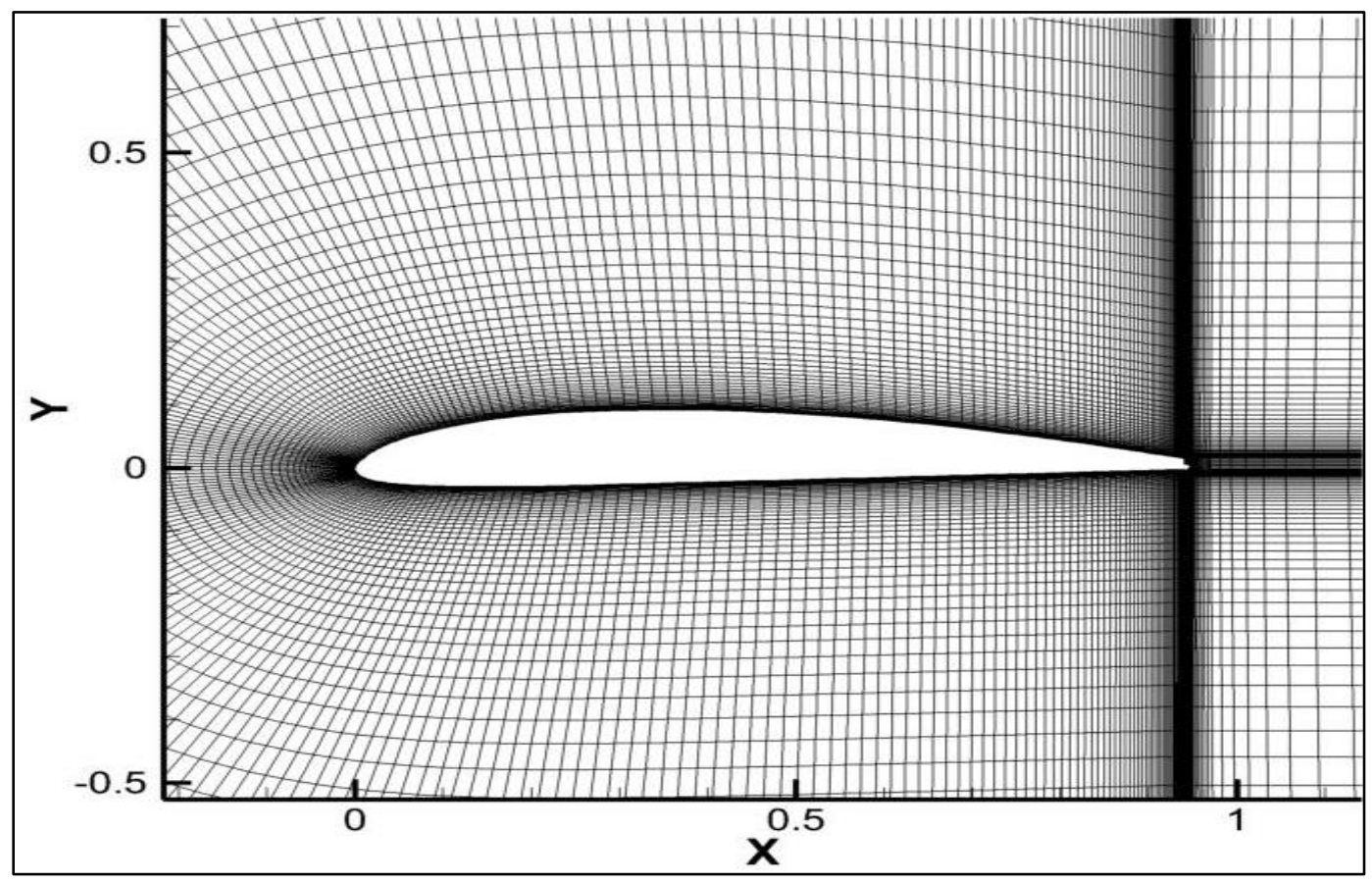

Figure 71. Close View of Airfoil Mesh Spacing for $C F D$ analysis

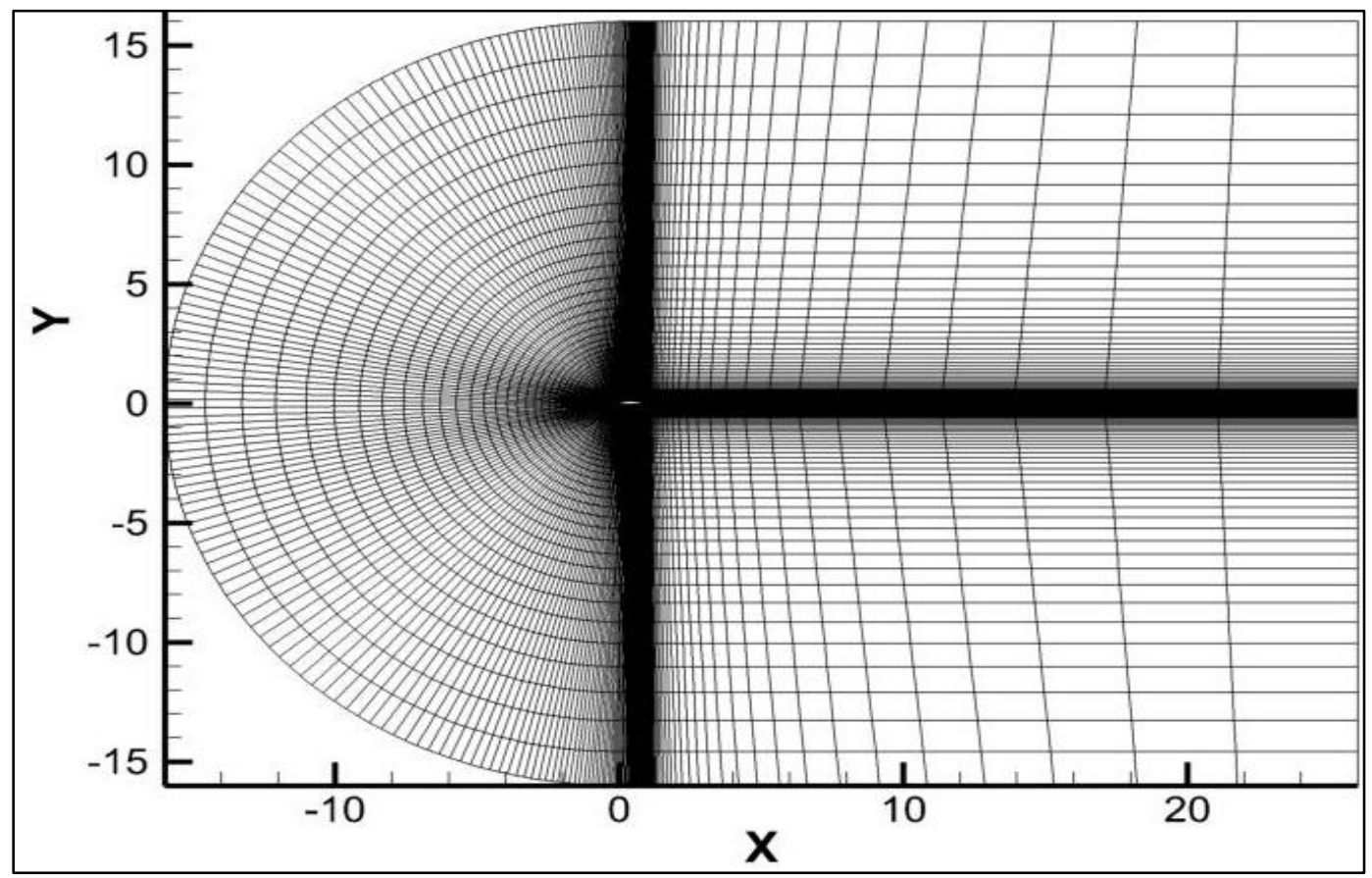

Figure 72. Complete CFD Analysis Grid Spacing

Initial trials and computational runs were used to show an estimated pressure distribution around the $C C$ airfoil at operating conditions. This was used to gauge the effectiveness of the grid spacing around the airfoil and to see that there were no unsolvable locations or error 
functions in the CFD program before running the entire simulation. In Figure 73, a pressure distribution at zero angle-of-attack is shown which appeared to resemble the expected look of an airfoil in this type of flow, high pressure at the stagnation point (red) and low pressure above the airfoil providing lift (blue). The stagnation pressure at the front section is highlighted as well as the pressure drop across the upper surface and pressure increase across the lower surface of the Clark-Y geometry, which provides the aerodynamic lifting forces. The trailing edge shows a pressure reduction around the rounded Coandă surface which is also expected, according to previous research and development in this area (Abramson, 2004, Englar, 2004)

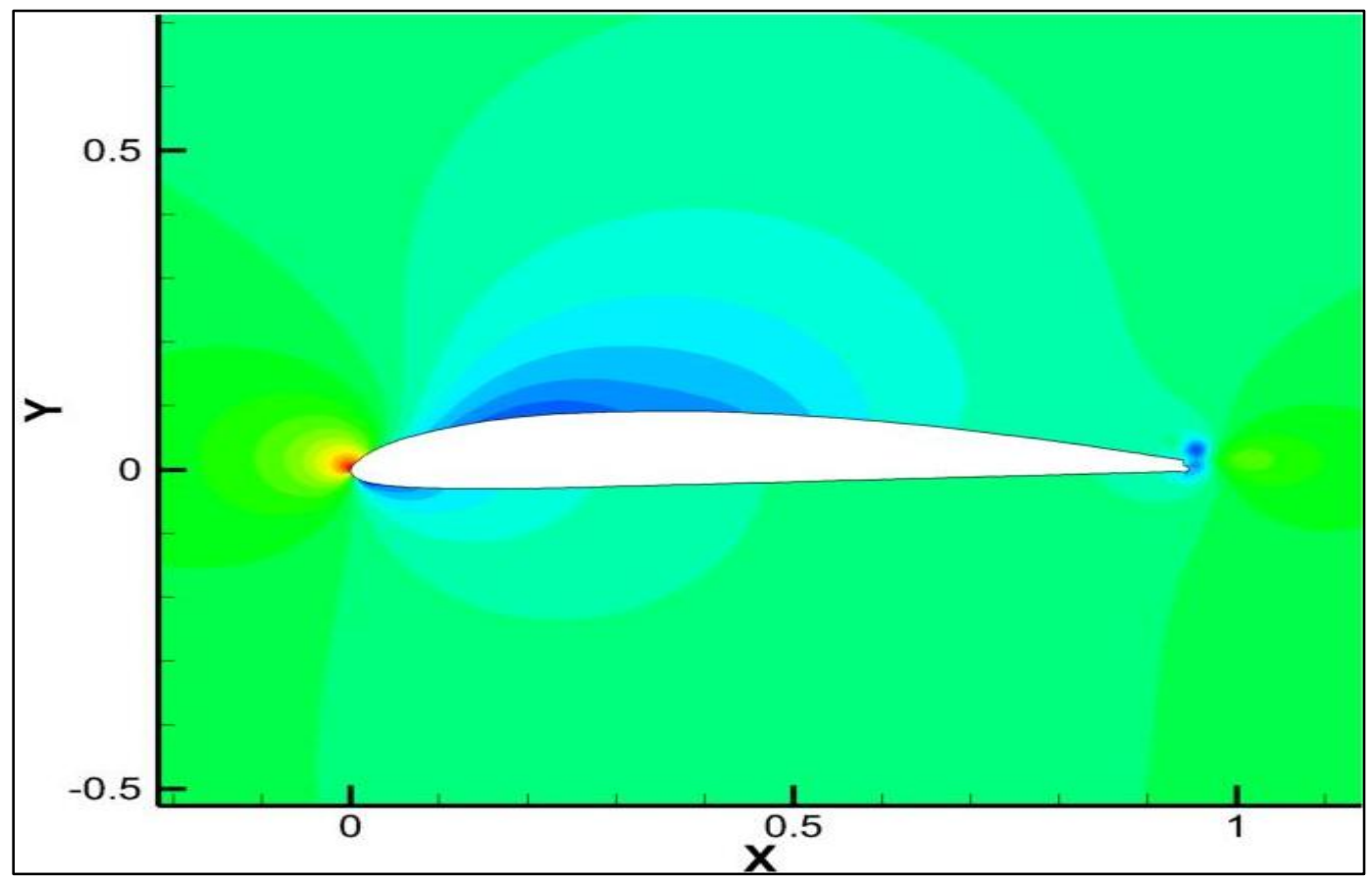

Figure 73. Initial Pressure Distribution of $C C$ Airfoil

\section{Computational $C C$ Airfoil Analysis}

The Computational modeling simulation software, Fluent, was employed to estimate the lift and drag force profile of the augmented airfoil section used in the design of the passively augmented circulation control propeller blade. This computational study was analyzed over a variety of simulated tunnel speeds, and rotational speeds, in order to study the effects of 
installation of a rounded trailing edge to the Clark-Y airfoil geometry. The results of this study were used in a blade element model to analytically study the circulation control propeller and to compare the physical wind tunnel tests to a computer simulation.

The experimental design in a computational environment, basically hinges on the test article geometry, and the solver settings used to simulate the aerodynamic flow past the model. In this case, standard sea-level air properties were used and various Reynolds Number simulations (specifically, 25,459; 63,649; and 127,298 - matching several experimental $R e$ values in the wind tunnel environments) were completed in order to accurately compare the computational data to the wind tunnel experimental data taken at $W V U$ and $A F R L$. Below in Figure 74, the airfoil velocity contours are shown at 20 degrees $A O A$ at a $R e$ value of 63649. There is separation predicted (blue velocity bubbles) due to the high angle-of-attack with respect to the freestream velocity. The computational experiment also took into account a necessity to study a full angle-of-attack sweep of the airfoil in order to match propeller geometries and inflow angles during simulation. 


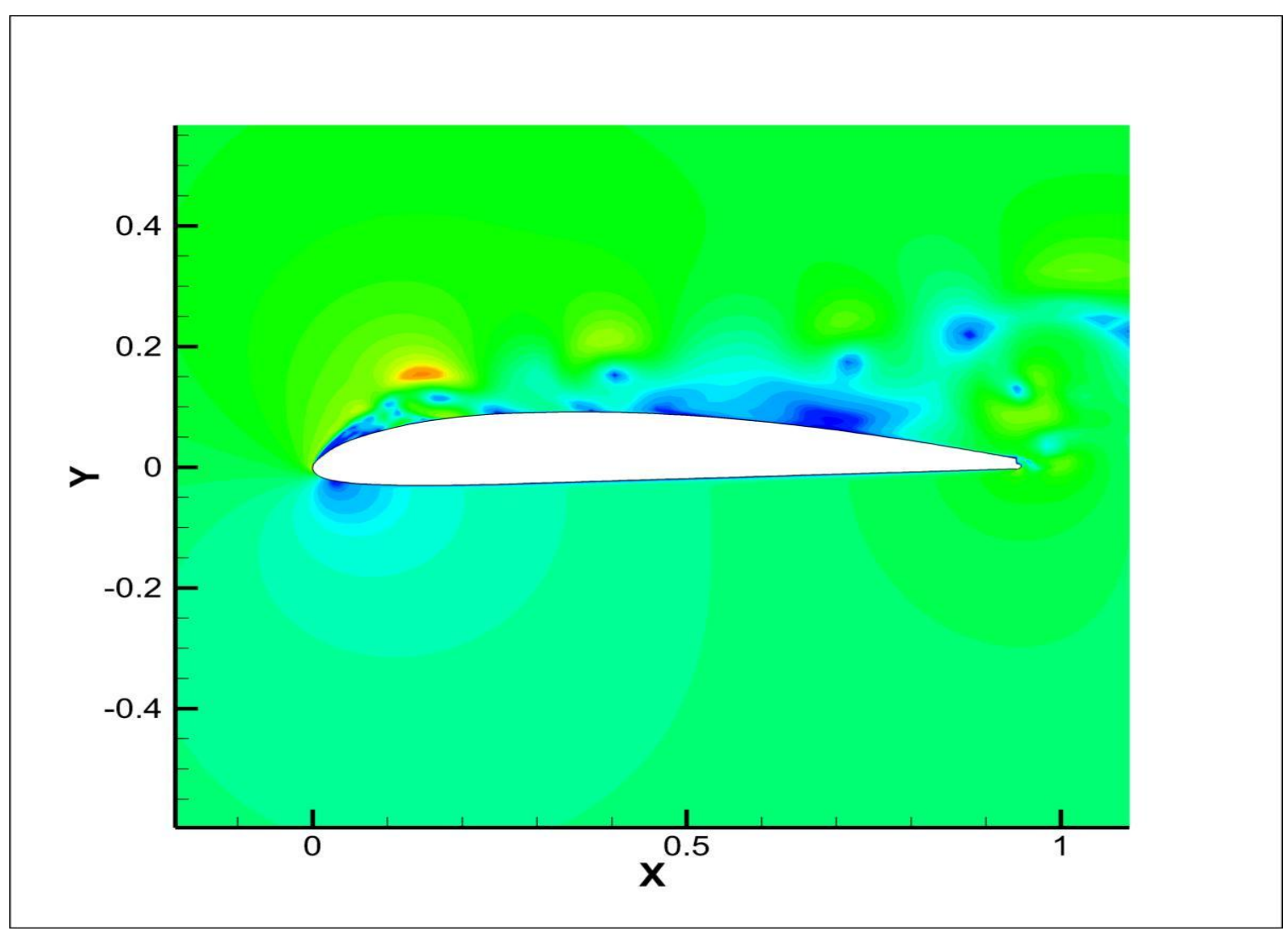

Figure 74. Velocity Magnitude of Airfoil Geometry at $R e=63649$ and $A o A=20^{\circ}$

In a similar simulation, the airfoil also predicts a series of separation bubbles at an $A O A$ value of -25 degrees, and $R e$ value of 63649. Shown in Figure 75, the separation bubbles (blue) are shown to be much more dramatic than the 20 degree $A O A$ case and are also found to have moved closer to the trailing edge on the under-side of the airfoil. 


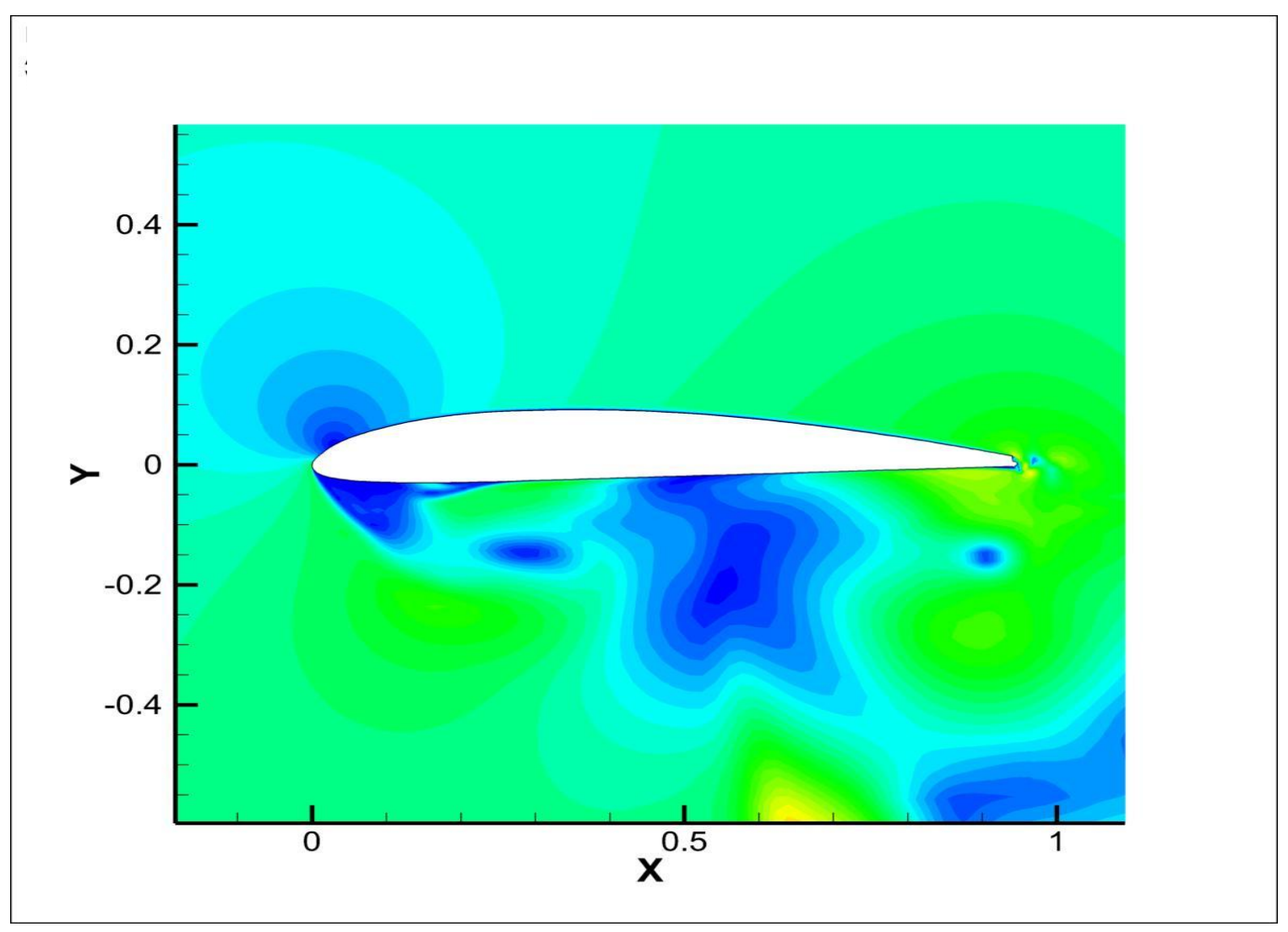

Figure 75. Velocity Magnitude at $R e=63649 A o A=-25^{\circ}$

Final results of the computational study, were reduced over time, and analyzed according to $R e$ and angle-of-attack of the airfoil. The results were averaged throughout the full expanse of time to converge on a solution for both section lift $\left(C_{l}\right)$ and section drag coefficients $\left(C_{d}\right)$. These values were used later in a blade element model to predict the output thrust and input torque needed to turn the augmented propeller. Along with the airfoil analysis of the unaugmented propeller, there blade element models were used to predict the performance of both propellers in relation to the experimental results shown from the wind tunnel testing.

Below, in Figure 76 the two-dimensional lift coefficient for the Clark-Y propeller geometry is shown over a range of Reynolds Numbers, including 25,495; 63,649; and 127,298, which in turn correspond to specific tunnel experimental values used in earlier sections. As 
expected, the results of the computational runs show an increase in overall lift coefficient for the airfoil geometry as the $R e$ value increases.

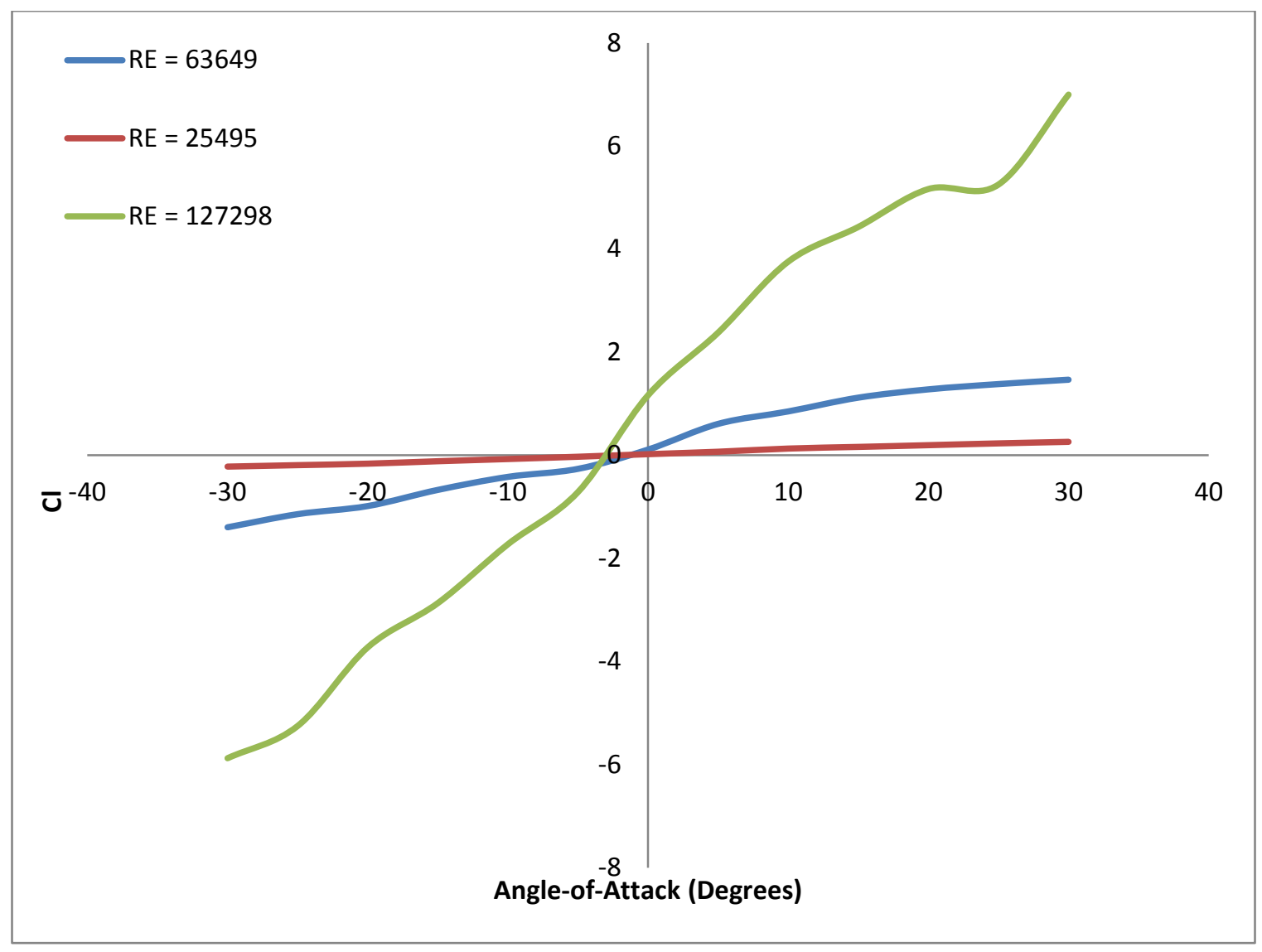

Figure 76. Circulation Control Augmented Lift Coefficient for Clark-Y Cross-Section

Similar results are shown below, in Figure 77, for the results of the drag coefficient study on the same airfoil geometry, operating at the same Reynolds Numbers. For each of the computational trials, a visible drag bucket is shown to occur at nearly 3 degrees $A o A$. These computational runs were conducted over the full spectrum of angles-of-attack of -30 to +30 degrees. 


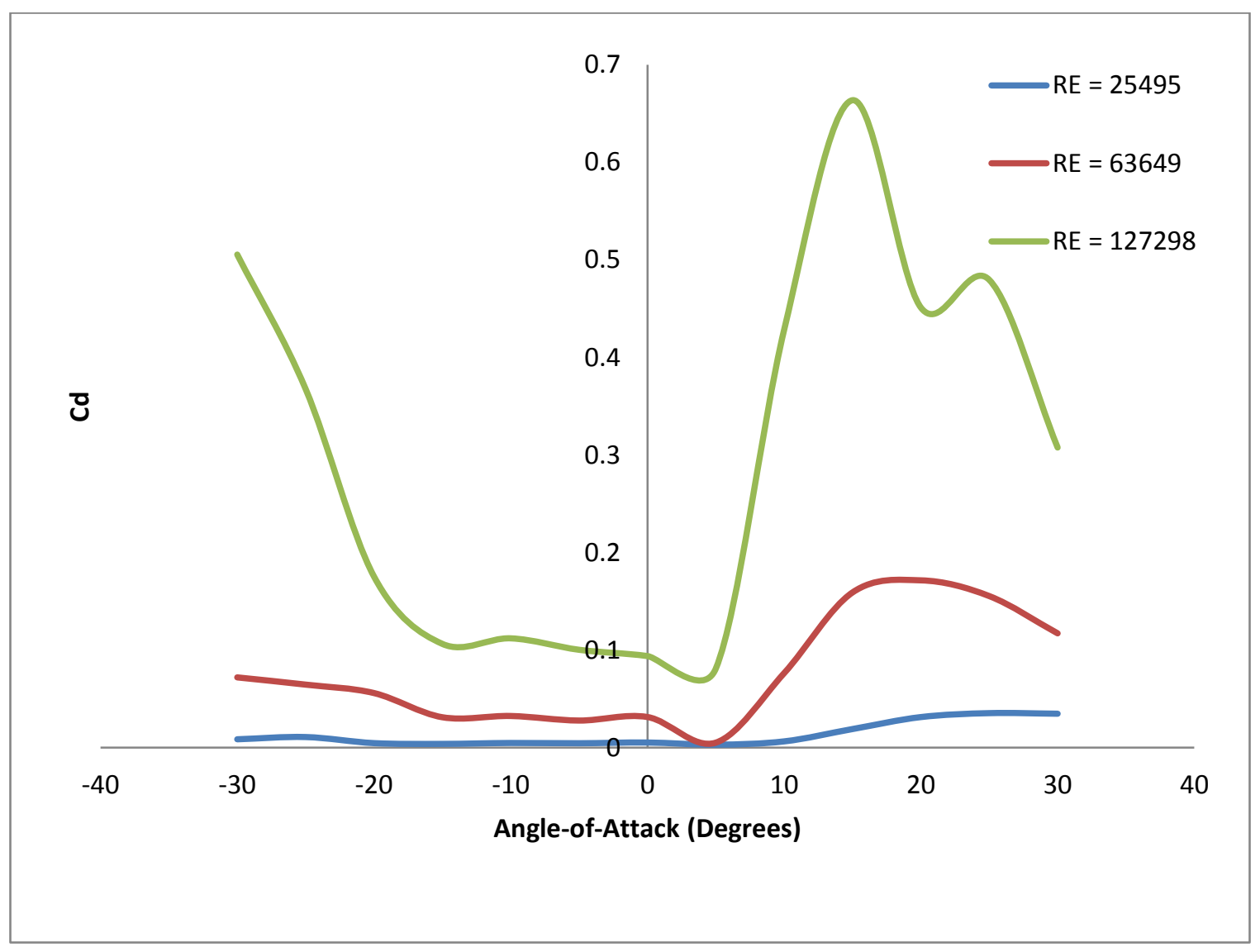

Figure 77. Circulation Control Augmented Drag Coefficient for Clark-Y Airfoil Geometry

The computational values studied for this augmented airfoil geometry were used in a blade element modeling of the entire propeller, factoring in the change in chord length due to the addition of a rounded trailing edge and $C C$ ejection slot to the airfoil over 33 percent of the radius of the propeller.

\section{Blade Element Analysis - Experimental Design}

It was also decided that a blade element model would be created based on the propeller geometry. As discussed earlier, there are generally two methods of analysis when it comes to the prediction of propeller performance, the actuator disk theory, and the blade element model. While the actuator disk theory provides a general approximation of the predicted forces that the propeller might encounter at operational conditions, this method ignores several aerodynamic principles, and is not powerful enough to provide an accurate estimate of drag forces on the 
propeller (Katz and Plotkin, 2001). Conversely, the blade element model splits the propeller into sections, as small as needed to be defined, and analyzes each section separately, as its own individual airfoil. This provides a more accurate estimation of propeller performance.

Generally, a blade element is measured based on the propeller characteristics and shape, the more cross-sectional areas that make up the propeller planform, the more elements would be necessary to achieve accuracy in predictive modeling. As the propeller used for this experiment was a fixed pitch propeller, it was chosen to split the elements at each 10 percent radial location, effectively providing 21 propeller elements for analysis (spread over two total propeller blades).

In preparing data for this analysis, it was necessary to estimate the overall aerodynamic capabilities of both propellers (two-dimensional lift and drag data) as well as exact geometry at each blade station (including span, chord, profile, twist angle, and $C C$ plenum dimensions). Several modeling software programs were used in this portion of the analysis, Pro-ENGINEER, for dimensional modeling, DesignFoil and XFLR-5 for aerodynamic analysis of the unaugmented propeller blade elements, and Fluent CFD modeling software to estimate the aerodynamic impact of adding a circulation control slot to the propeller blade.

First the propeller was found to have a constant cross sectional geometry, a Clark-Y profile, which allowed for a simple analysis in DesignFoil and XFLR-5 software packages throughout a range of Reynolds Numbers. The aerodynamic characteristics are found later in Appendix G - Clark-Y Simulated Airfoil Data, specifically for a range of Reynolds Numbers encountered during experimentation. A range of angles-of-attack ( -20 to 20 degrees) were analyzed in DesignFoil, and a full spectrum of angles (between -180 and 180 degrees) were analyzed in XFLR-5. This data, coupled with the propeller dimensions, measured in ProENGINEER and the computational results studied based on the $C C$ propeller blade geometry, 
gave all the necessary aerodynamic input data needed to produce a reliable blade element for the unaugmented propeller.

\section{Summary}

Experimental setup of a propeller test apparatus can be achieved in many different ways, a wind/water tunnel is not a necessary piece of hardware anymore. Computational methods have gained a foothold in the experimental community and can be considered accurate predictive tools, assuming that the results can be compared with previous experiments or historical data. The propeller is a highly studied historical component in which aerodynamic forces, lift, drag, and moments have all been readily characterized. It has even been shown that prediction of forces is accurate if the geometries of the propeller, blade span, radius, rotational speed, and operating conditions, are known.

In order to experiment on the proposed passive circulation control system, and study the effects it has when added to a propeller, it will be convenient to choose a propeller currently in use by $U A V / A U V$ 's and perform a series of computational tests in a flow visualization software package. Using results from the preliminary sensitivity study, and comparing them to a previous experiment on the same currently used propeller, a new propeller began to take shape by varying and testing several input parameters of the passive circulation control propeller.

This method includes slot height, plenum pressure, capture device area and nominal operating conditions. With these results, a scale model was produced in order to physically test the propeller, and later validated with computational simulations. This physical model was mounted in a wind tunnel, and aerodynamic forces on the propeller were measured. This method allowed for the theory of a new design to take shape, and accurately showed proof-of-concept while allowing for computational simulations to further enhance the design and to consider each condition as its own unique set of trials. 


\section{Experimental, Computational, and Analytical Results}

This section will explain the results of the propeller experimentation as well as the corresponding computational study. Experimental data was taken in a wind tunnel environment at several rotational speeds and forward velocities. These experiments were conducted in a wind tunnel at West Virginia University ( $W V U$ ), Morgantown, West Virginia, as well as the vertical wind tunnel at Wright-Patterson Air Force Base/Air Force Research Laboratory (WPAF/AFRL), in Dayton, Ohio. Computational studies were completed both as a data analysis program written in Microsoft Excel and as a visual flow study in Fluent CFD software and correlated to both experimental and theoretical results. The relationship of the data between theoretical, experimental, and computational provided a source of accuracy for determining the applicability of this system as well as proof-of-concept.

\section{Experimental Results: Wind Tunnel Analysis}

The most interesting results that were recorded while testing this circulation control augmented propeller was the prospect of increasing the thrust of the propeller and/or decreasing the required amount of input torque on the propeller and in turn the motor driving the propeller. These interests have the added benefit of being able to save money in fuel costs by increasing the overall range of the aircraft.

\section{Static Propeller Analysis}

The first variable that was examined was the thrust capabilities of the augmented and unaugmented propellers at static conditions. This gave a starting point for what kind of performance the propeller can be predicted to have while in operation. It also is necessary to have the static data on file as that is the industry standard on how to size the propeller according to what kind of payloads and flying characteristics are predicted. In Figure 78, the theoretical 
calculations for thrust of a propeller are given in relation to the actual tabulated values taken during experimentation, both at $W V U$, and in the $W P A F B$ experimental tunnel. In this analysis, the trends of the data are shown to agree, however the simplistic view of the theoretical static loading equations do not take into account boundary layer effects and slipstream offset for the end result. As a result, the theoretical calculation of static loading on a propeller is an overestimated result, used in industry to accurately size motors and engines off-the-shelf to a particular propeller function, with a standard error factor already included in the sizing convention.

When evaluating the augmented and unaugmented propellers with respect to the specific tunnel the experiments were conducted in, the accuracy of the data collected at WPAFB is shown to be closer to the theoretical calculations, specifically because of the quality of the hardware used while testing. Because the experimental set-up at $W V U$ used a load cell to measure the thrust under static conditions, a larger amount of experimental precision error is expected due to the inherent lack of dynamic accuracy in the load cell.

These types of devices are not meant to take accurate readings under unsteady loadings, i.e. rotating propellers. However, with that said, the maximum value of the difference in thrust measurement between the theoretical and the experimental values measured in the $W V U$ wind tunnel was measured to be about 14.5 percent of full scale at $2500 \mathrm{rpm}$. The WPAFB data showed a maximum difference of 8.0 percent of full scale at $3500 \mathrm{rpm}$. As a relation, the maximum difference in static thrust between the $W V U$ wind tunnel tests and the WPAFB wind tunnel experiments was only 6.5 percent of the full scale measurements at $2600 \mathrm{rpm}$. 


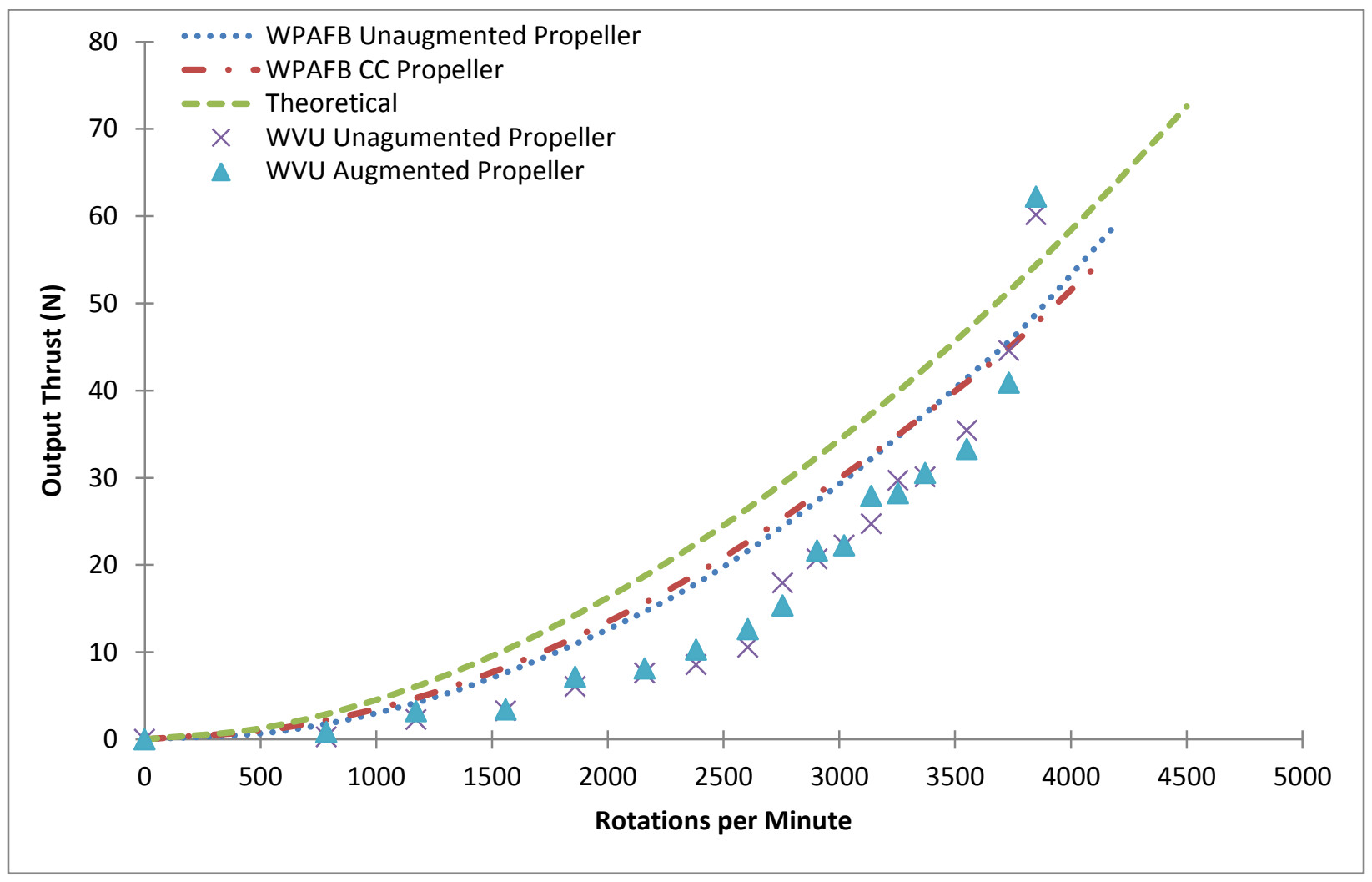

Figure 78. Theoretical Static Loading Compared to Experimental Results

Static propeller characterization allows for a manufacturer to use maximum allowable thrust settings to size a propeller to a specific need or application. These types of specifications are useful when choosing a motor or engine to mount with the propeller and aircraft. It is necessary to know the maximum capabilities of the propeller before sizing an ample power supply for the craft. As an example of this, the maximum output thrust for the original Zinger, Inc. propeller, according to the manufacturer was approximately $60.0 \mathrm{~N}(13.5 \mathrm{lb})$, consequently, the motor chosen to drive the experimental analyses as well as the mounting sting and force calibration apparatuses were sized according to this output force.

\section{Wind Tunnel Testing Analysis}

As previously explained, it was necessary to split the wind tunnel testing scenarios into two separate experiments due to the allowable tunnel speeds with available hardware. The first section of data was collected at $W V U$ in a specifically design and constructed wind tunnel. With 
the variance of rotational speeds and tunnel velocities available, advance ratios up to 0.45 were able to be collected with reliable repeatability for both propeller experiments. The second set of trials was conducted at the vertical tunnel at $W P A F B$ in Ohio where increased tunnel velocities were achievable. These experiments collected data for advance ratios from 0.40 to 1.20 , overlapping some of the previously taken data for completeness.

For the experimental runs, data for thrust force and input current were the main factors taken into account. These values were used to study the overall performance of the propeller in both aerodynamic performance (thrust and torque required) as well as overall efficiency (based on input and output relationships). Between the two propeller geometries and testing scenarios, the thrust forces are shown to be similarly linked. However the experimental data for the amount of input current needed to turn the propeller is higher for the unaugmented propeller in comparison to the augmented case. This suggests that the overall lift of the propeller blades has remained relatively constant between the two blade planforms, and the drag values of the augmented propeller have been reduced with the pumping of the stagnation air for use in circulation control.

Below, in Figure 79 and Figure 80, the experimental efficiencies are shown for the unaugmented propeller as well as the augmented propeller, as examined in both the $W V U$ wind tunnel (low range of advance ratio) and the WPAFB vertical wind tunnel (higher ranges of advance). These plots show the output results of the trend studies of the two propellers over two different testing conditions, and show conformity between the two. Because the propeller stopped producing thrust at an advance ratio of 0.60 , the results found were for what would be termed a "wind-milling propeller." This began the area of performance where the propeller no longer produces thrust power, but began adding power back into the system. 


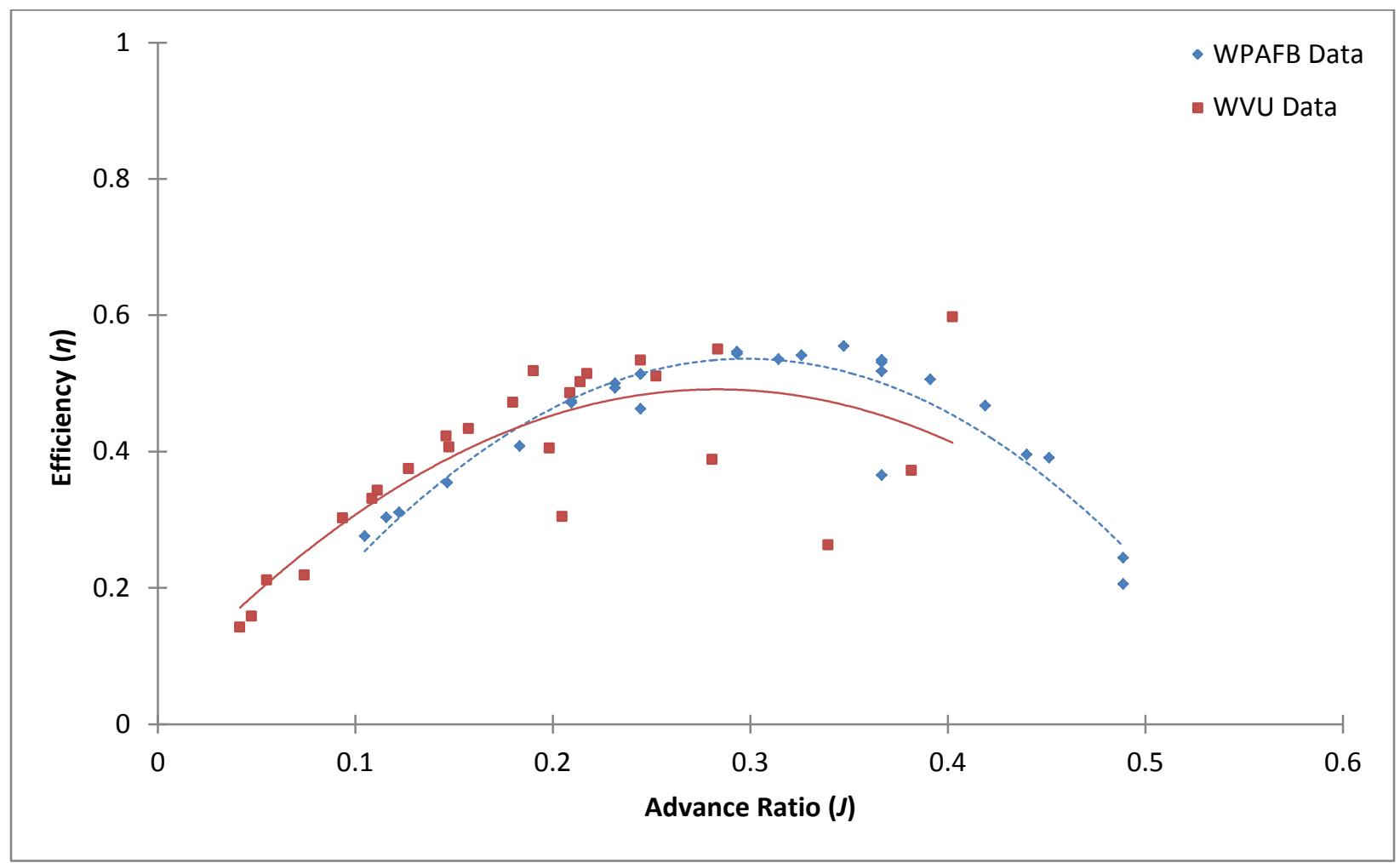

Figure 79. Unaugmented Propeller Efficiency Performance Data

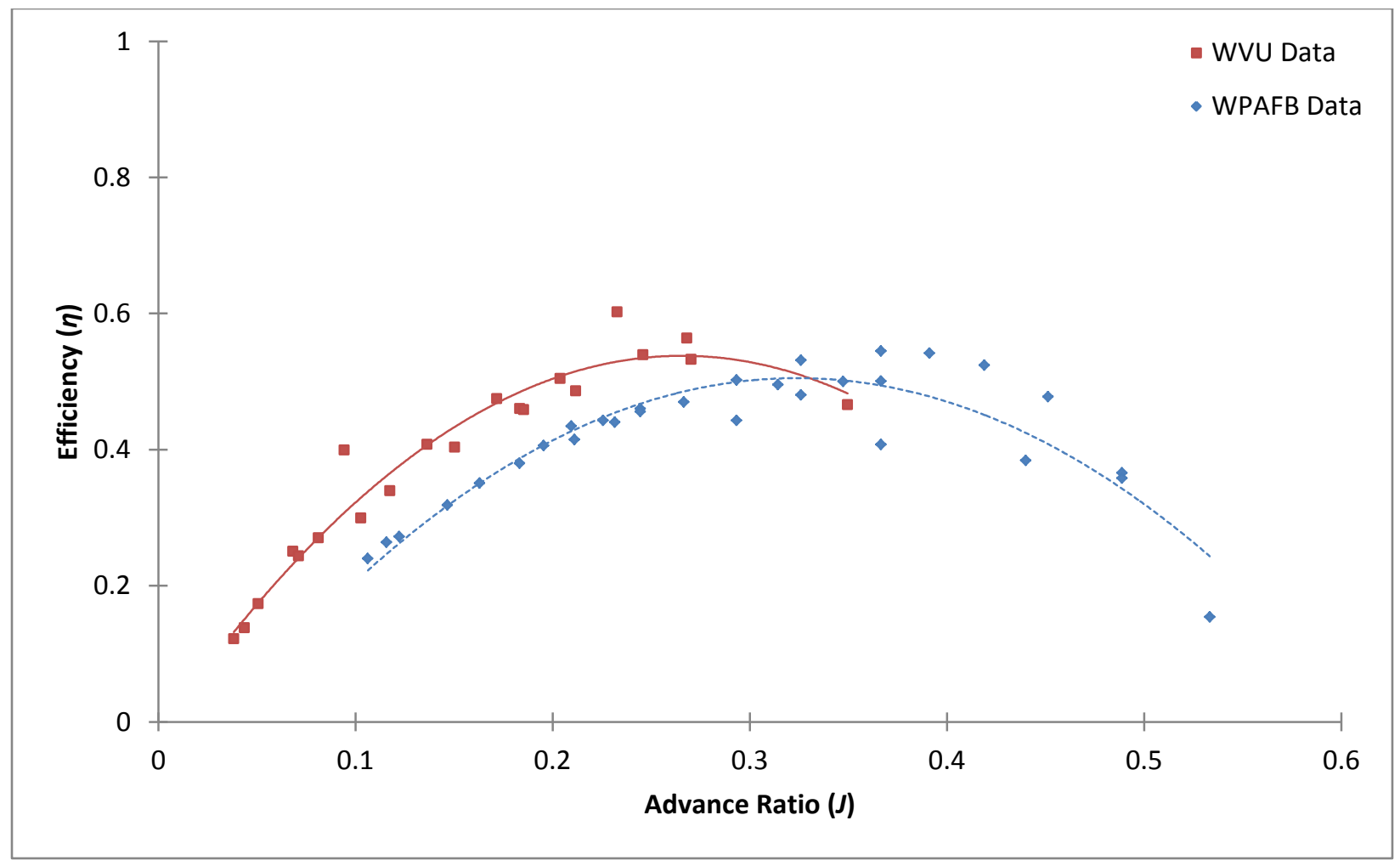

Figure 80. Augmented Propeller Efficiency Performance Data 
In Figure 81 the measured thrust forces during experimentation are shown as nondimensional values $\left(C_{T}\right)$ with respect to the corresponding advance ratio of the propeller. The trends for both propellers show a second order fit decline in output thrust as advance ratio increases and show a net negative thrust output leading to power being taken from the freestream velocity, much like a windmill or wind turbine type of effect. Experimental results show that the output thrust force of the augmented propeller is equal to or slightly more than the baseline unaugmented experimental propeller at all advance ratios. In specific tested sections between advance ratios of 0.15 and 0.25 the two resulting thrust capabilities are very close to being equal, with the augmented propeller measured to be slightly higher, measuring as much as 5.0 percent more.

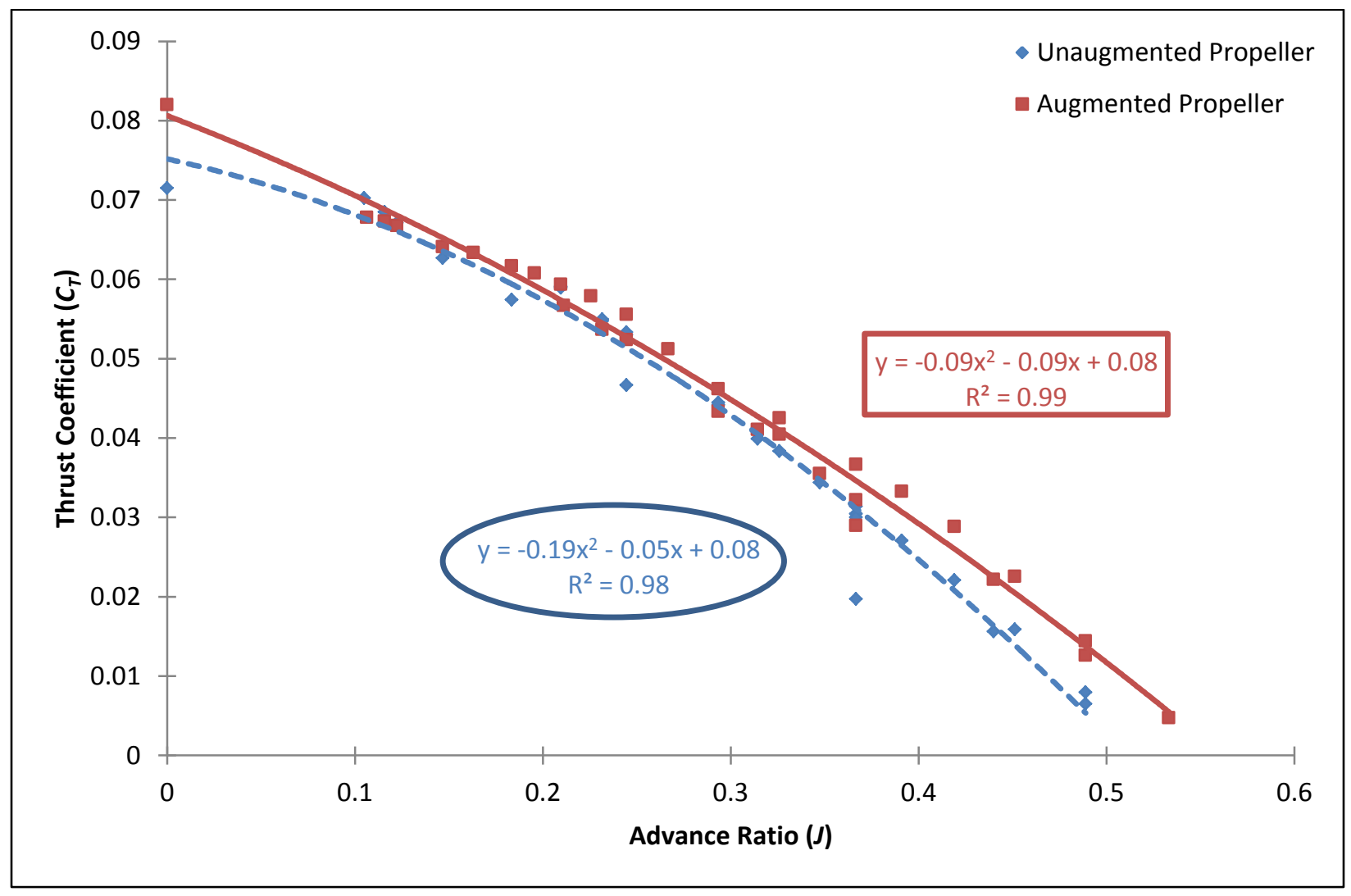

Figure 81. Output Thrust Coefficient Comparison Between Augmented and Un-augmented Propeller Experiments - WVU and WPAFB Reduced Results 
In Figure 82 the input current measured for each advance ratio of the propeller tests was converted into a power consumed by the motor in order to rotate the propeller to a certain rotational speed. The trend study shows that at low values of advance ratios, the input torque needed to turn the augmented propeller is 10.0-12.0 percent more than that of the baseline propeller. Again as the advance ratios begin to approach 0.50 , the augmented propeller begins to show a 8.0-10.0 percent increase in required torque, making it feasible to increase the overall performance of the aircraft which uses the $C C$ propeller. As the advance ratios begins to approach 0.60 , where the propeller ceases to make thrust, the input torque required to turn the propellers begins to equalize and show little deviation from one another.

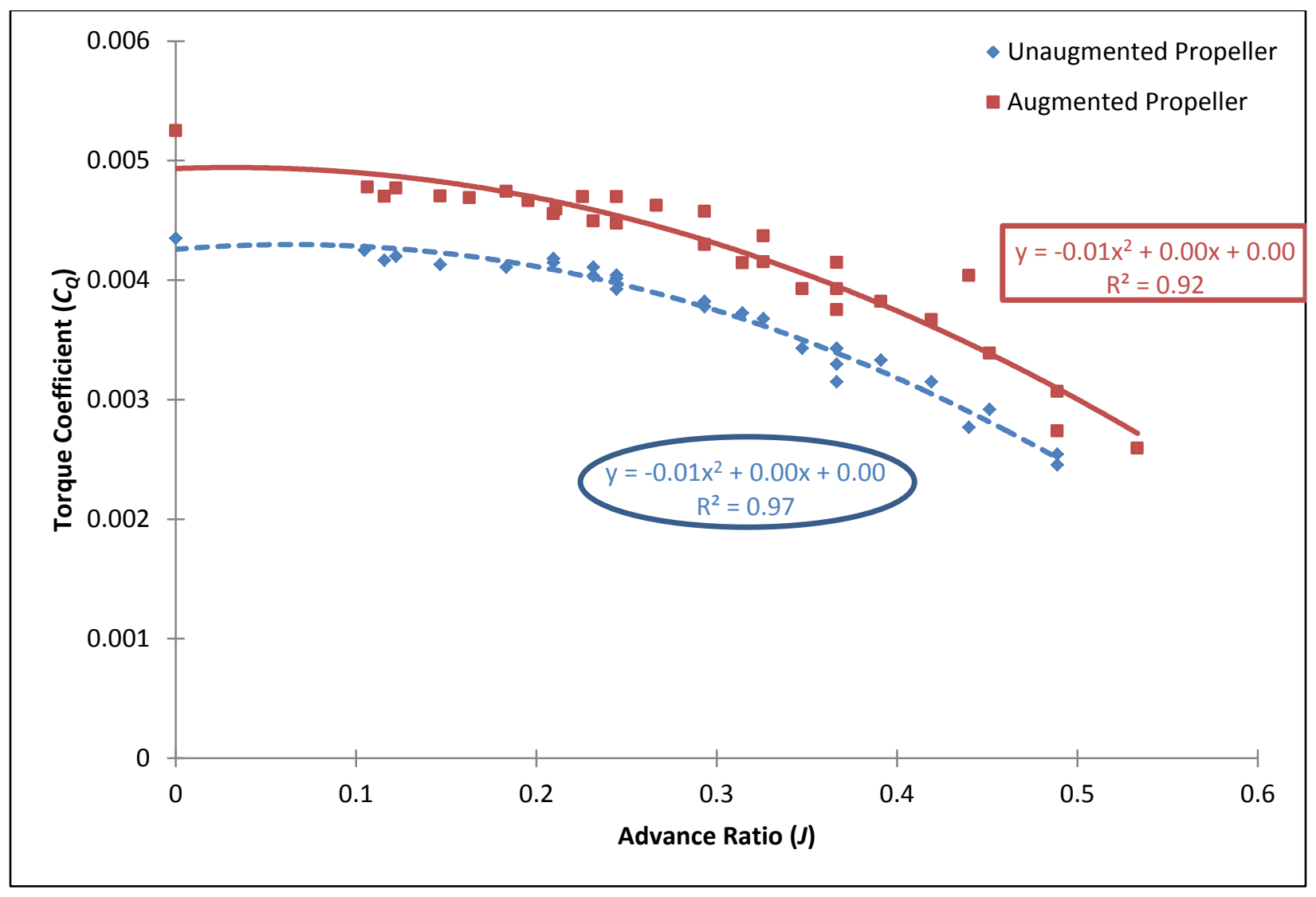

Figure 82. Input Torque Coefficient Comparison Between Augmented and Un-augmented Propeller Experimental Data - WVU and WPAFB Reduced Results 
This portion of the experiment shows the overall efficiency of the circulation control propeller increases due to the small increase input torque required to turn the propeller as well as the overall increase in output thrust measured over the medium range of values of advance ratios (0.25-0.5). As the advance ratios climbed above 0.60 during experimentation, the overall efficiency ratings of the two tested propellers showed equality. Noted, however, was data collected at values of advance ratios above unity, the thrust forces measured began resulting in negative values, and thus began the section of the propeller flight envelope where the aerodynamic forces transitioned into a "windmill" type of loading.

This is when the propeller is so overloaded it begins taking power from the free-stream velocity, rather than energizing it. Below, in Figure 83, the overall non-dimensional power coefficient is shown with respect to advance ratio and shows a relatively consistent 8.0-10.0 percent difference in required power to run the propeller. This is expected as the power coefficient is based on the input torque needed to turn the propeller. The calculation of this data made overall efficiency of the propeller easier to calculate, based on the ratios of input power and output thrust. 


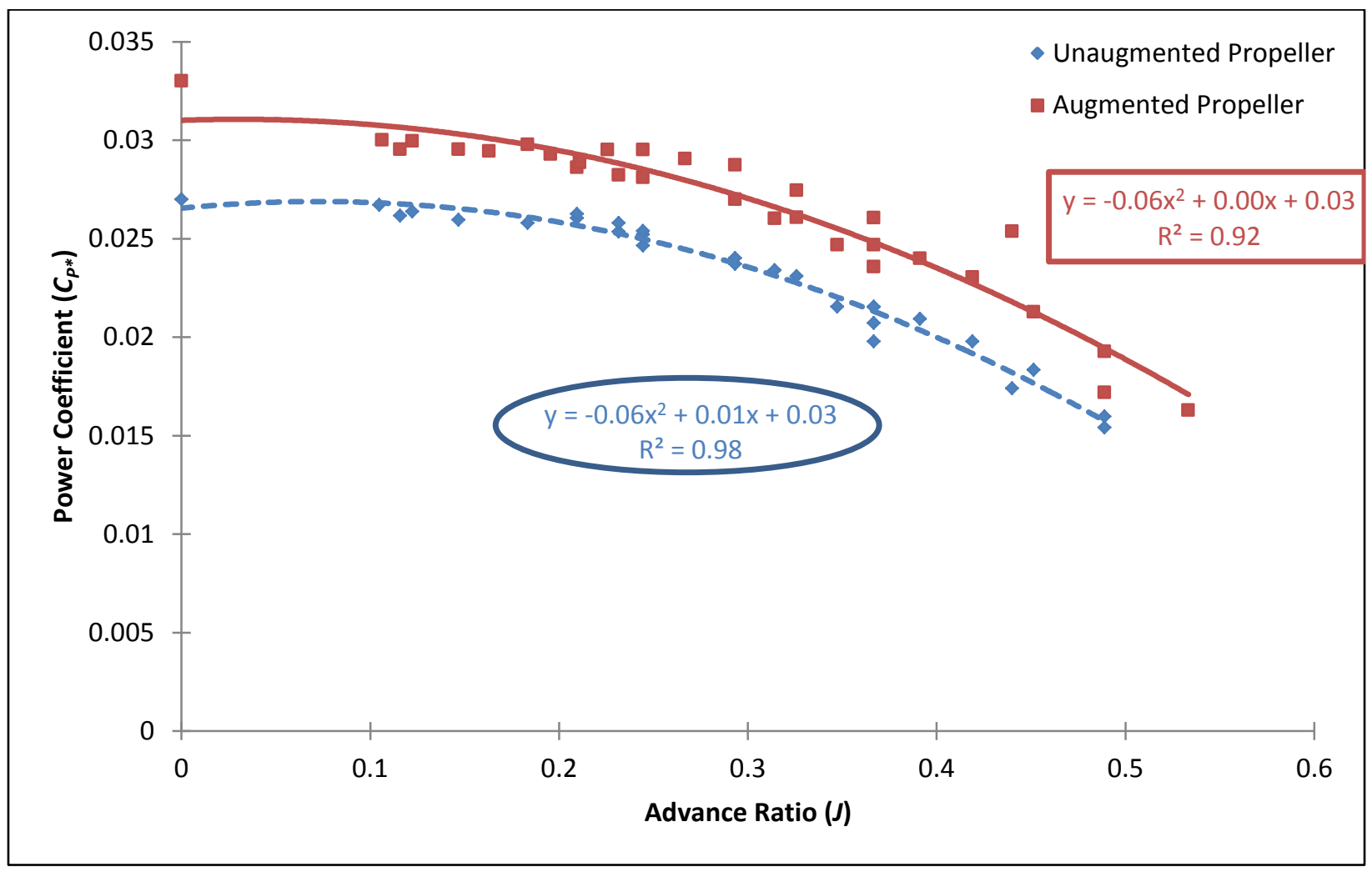

Figure 83. Power Coefficient Variation across Advance Ratio - WVU and WPAFB Reduced Results

The data from the two experiments was reduced together, allowing for a piece-wise trend study of the overall effect of circulation control on the prototype propeller from a coefficient and an efficiency standpoint. For the coefficient data, the information was reduced into Figure 81, Figure 82, and Figure 83 removing the repeating information between the two experiments via averaging, and providing a cleaner overall plot of the data. The overall plotted data without simplifying the data sets and reducing the number averaged data is given in Appendix $\mathrm{K}-\mathrm{Raw}$ Data from Wind Tunnel Experiments.

Similarly, the overall propeller efficiency, shown below in Figure 84 with respect to experimental advance ratio was estimated across both experiments in both wind tunnel environments. The low end (0.0-0.4) of the advance ratio values were able to be gathered in the wind tunnel experiments at $W V U$ while the larger values of advance ratio (0.45-1.2) were 
gathered while testing at WPAFB. Together with the full spectrum of thrust coefficient $\left(C_{T}\right)$, power coefficient $\left(C_{P^{*}}\right)$ and torque coefficient $\left(C_{Q}\right)$ data, and overall efficiency $(\eta)$ prediction was tabulated for both the augmented and un-augmented propeller.

The use of the advance ratio of the propeller takes the output results of the experiment to a non-dimensional value and allows for comparison of data between any number of different blade types, forward velocities, and thrust forces. As shown in Figure 84, the overall efficiency of the two studied propellers does not seem to change between the advance ratios of 0.0 and 0.30 , with the unaugmented propeller being just a fraction of a percent better overall.

However, at advance ratios between 0.31 and 0.55 , the experimental values of the augmented propeller show a better performance factor and higher efficiency ratings. Using an integration of the estimated curve of efficiency performance, the overall change in efficiency across the entire range of advance ratios is estimated to be 5.74 percent better using the $C C$ propeller.

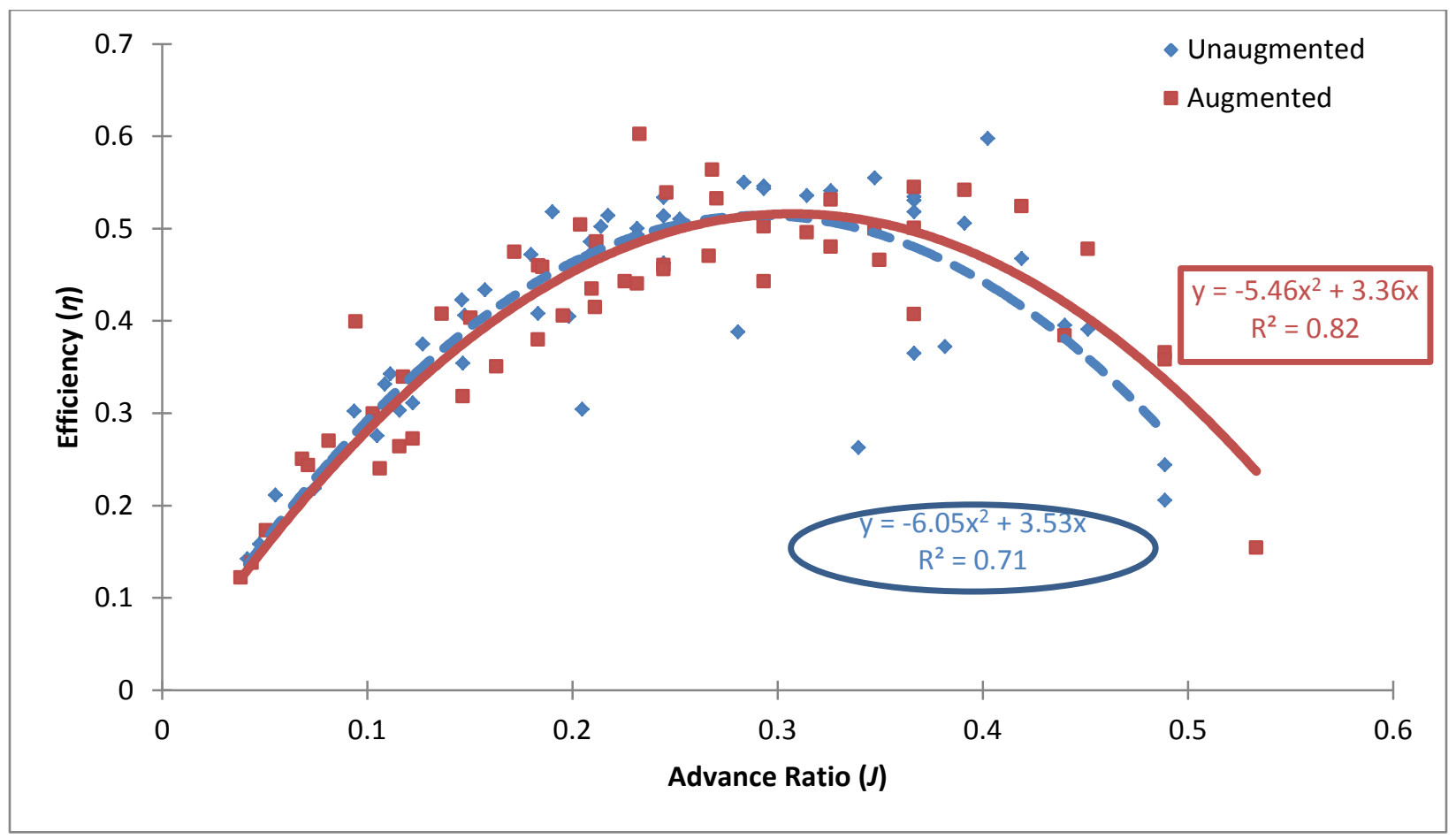

Figure 84. Full Spectrum Propeller Efficiency with Respect to Advance Ratio 
Secondary testing scenarios of inserting the propeller into the free-stream velocity at a known angle-of-attack were conducted at $W P A F B$ to simulate the effect of $C C$ during a rapid dive or climb maneuver. This test was meant to explore the effects seen in flight envelopes other than the gradual changing types of free-stream angle-of-attack, normally seen at take-off and landing conditions (i.e. dog-fighting).

Here, as shown in Figure 85, the overall propeller efficiency ratings have been tabulated as the propeller experiments were cycled through a series of angle-of-attack settings in the WPAFB Vertical Wind Tunnel. The propeller was first tabulated at zero $A o A$ with respect to the freestream showing the overall efficiency rating. Then at increments of five degrees, the propeller was moved relative to the freestream and tare values were taken in relation to the gravitational effects of acting on the force balance. The overall performance of the propeller actually seems to increase with angle-of-attack, at advance ratios between 0.20 and 0.35 .

However, as the advance ratios of the propeller climbed above 0.35 , the overall performance of the propeller begins to show a decline as $A o A$ increases. This could be due to the inflow angles of the propeller plane changing as the entire propeller varies in $A o A$. As this experiment was merely a precursor to future design considerations, it was only tabulated once in the wind tunnel environment, and will need to be revisited in another study before an accurate prediction can be made. 


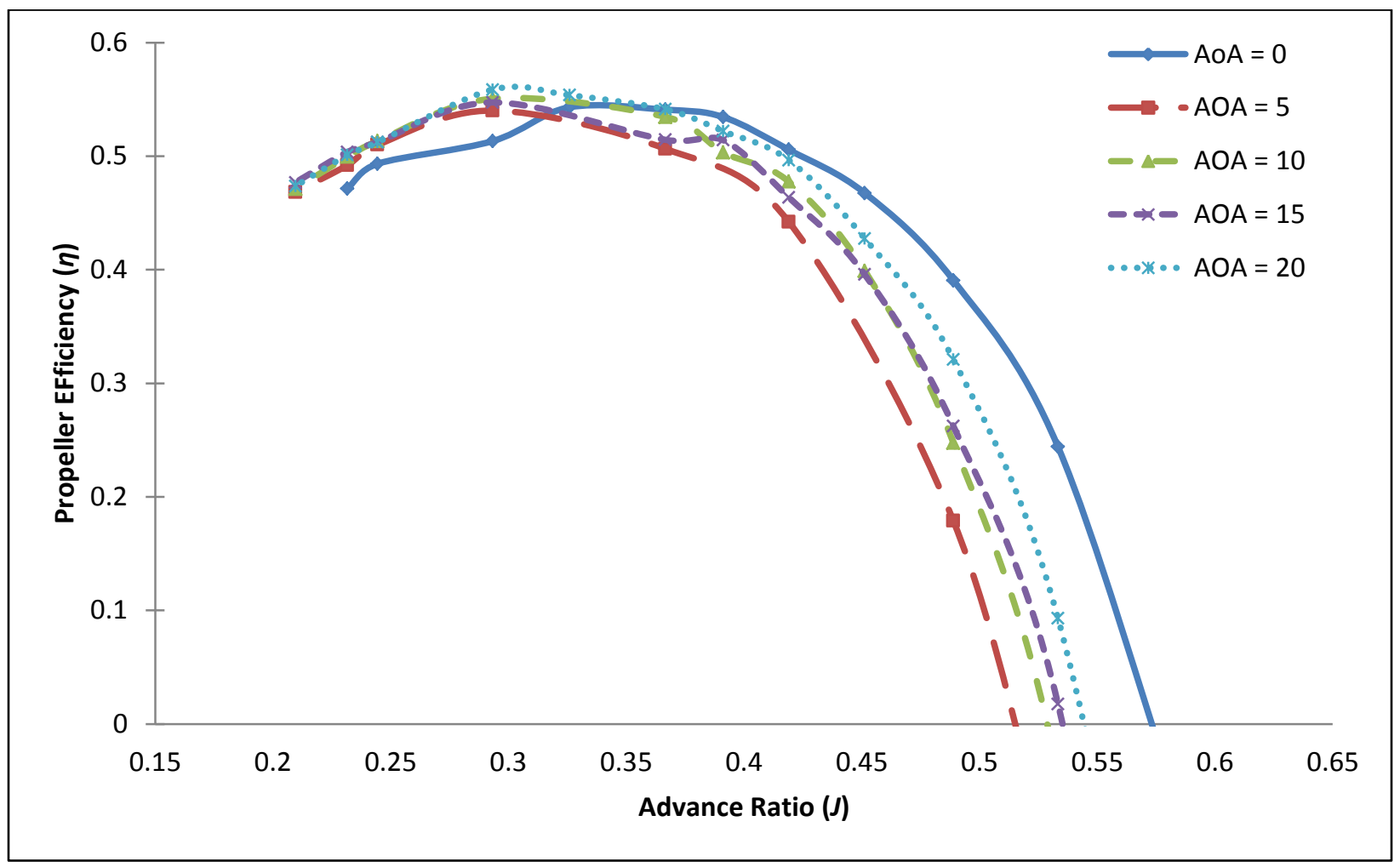

Figure 85. Effect of Propeller Efficiency as Angle-of-Attack Changes

Full reduction of data from experimental runs in the $W V U$ wind tunnel as well as the WPAFB vertical tunnel can be found in Appendix I - Experimental Thrust Comparisons, Appendix J - Experimental Torque Comparisons, and Appendix L - Castle Link Speed Controller Data Logger. In the WPAFB tunnel, while testing there was a data logger attached to the speed controller which was able to output a variety of data during each trial run, including measured rotational speed of the propeller, input power, ESC temperature, input current, input voltage, and throttle setting. The data logger software was then used to plot these values with respect to time, providing an overall test matrix $\log$ of the experiment and most importantly, a secondary set of measured data to compare to the tunnel data acquisition system.

The final testing scenario was conducted during the previous wind tunnel experiments at $W V U$ in an attempt to visualize the effect of ramping the propeller through the entire spectrum of rotational velocities of the motor while at a fixed tunnel velocity. This study was conducted with 
experimental prototype, at two different tunnel velocities. Also, the tests included both ramp-up of the rotational speeds $(0-3800 \mathrm{rpm})$ as well as ramp-down $(3800-0 \mathrm{rpm})$. This experiment was not part of the original testing plans but it was hoped that it would provide a starting point for dynamic simulation or the response of the prototype propeller, and should be considered for future study.

For the first set of conditions, the $W V U$ tunnel fan was set at $60 \mathrm{~Hz}$ which was the maximum input from the motor controller and corresponded to a tunnel head of 10.0 meters per second (22.4 miles per hour) free-stream velocity. The second set of data points were taken at 43 $\mathrm{Hz}$ fan input which corresponded to 7.0 meters per second (15.7 miles per hour) tunnel velocity. The data runs were taken over time to show the change in output thrust and input torque to the propeller as the rotational dial was ramped fully through the spectrum of controllable inputs.

Below, Figure 86, and Figure 87 show the differences in thrust output and torque input, respectively, seen by the propeller prototype and experimental equipment while cycling through the ramp down process (i.e. full speed $\mathrm{rpm}$ down to $0.0 \mathrm{rpm}$ ). It is important to note that the controllability of the motor input in this case is based on human input, and was therefore very difficult to try to match the rate that the motor rotational speed was reduced.

While Figure 87 shows a relatively predictable input torque value due to the input current applied to the motor, Figure 86 shows much more sporadic thrust values at the higher tunnel speeds. The inability to show an accurate generalized trend might suggest that the sampling rate was not fast enough or there was some flow inconsistencies present while experimenting. Another interesting note is that at higher calculated advance ratios, 1.0-1.40, thrust is shown as a negative value, suggesting that the propeller is no longer functioning as a thrust mechanism but as a "windmill" and beginning to retard the flow, and take power from the free-stream velocity. 


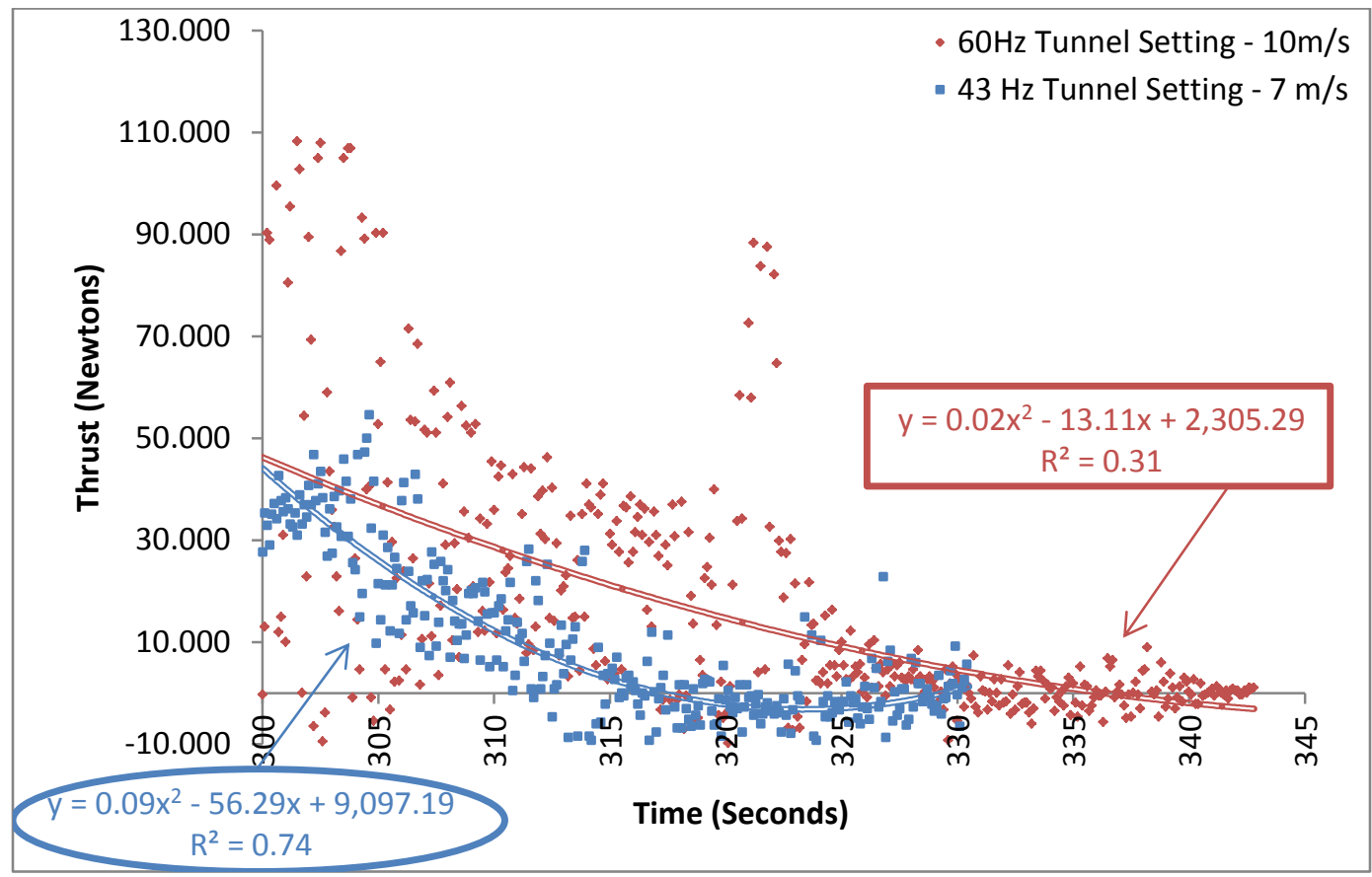

Figure 86. Full-Scale Ramp-Down Thrust Comparison with Respect to Time

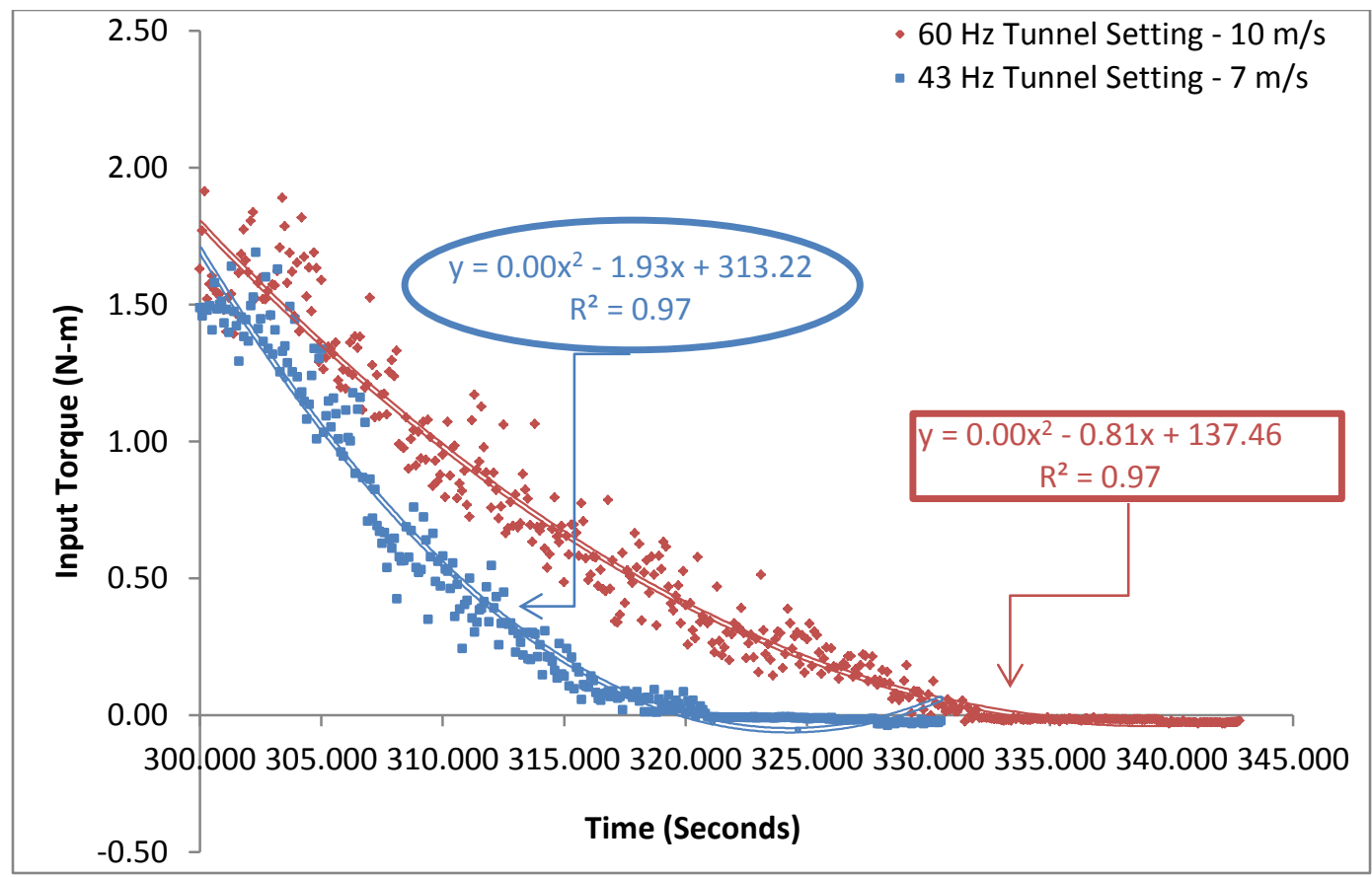

Figure 87. Full-Scale Ramp-Down Torque Comparison with Respect to Time

Similar to the previous experiment, tunnel fan conditions were set to $60 \mathrm{~Hz}$ and $43 \mathrm{~Hz}$, corresponding to 10.0 and 7.0 meters per second tunnel head. For this instance, the rotational speed was varied through the overall range from zero to maximum $(3800 \mathrm{rpm})$ and was plotted 
as a function of time. Here, again, the quick study shows that the input torque value on the propeller (Figure 89) can be accurately predicted at both tunnel speeds while ramping the drive motor rotational speeds from rest to maximum rpm. However, as seen in the ramp-down experiment the thrust forces (Figure 88) are less predictable over the higher tunnel velocities, again suggesting that the sample rate was not high enough for dynamic experimentation while collecting load cell measurements.

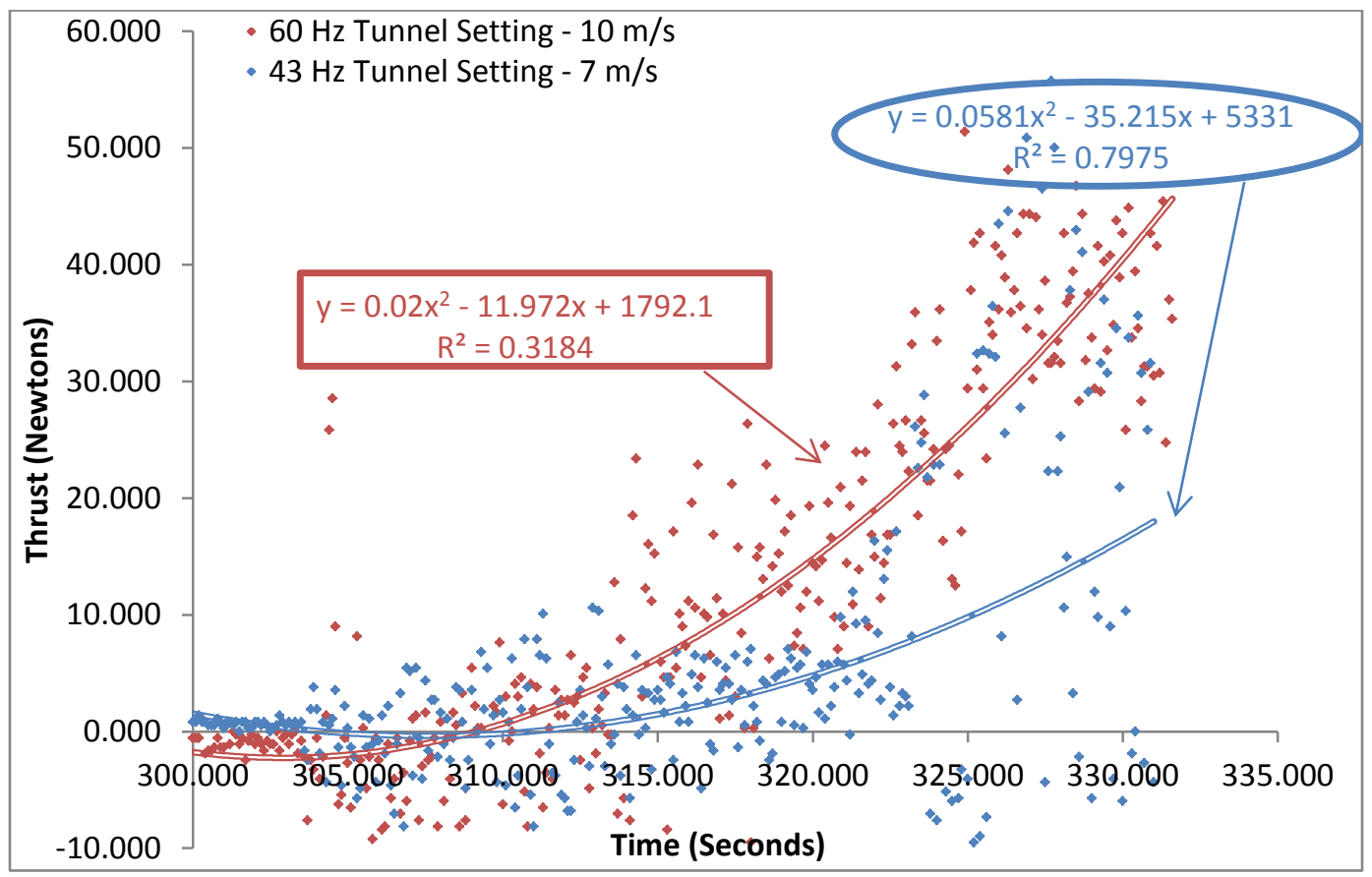

Figure 88. Full-Scale Ramp-Up Thrust Comparison with Respect to Time 


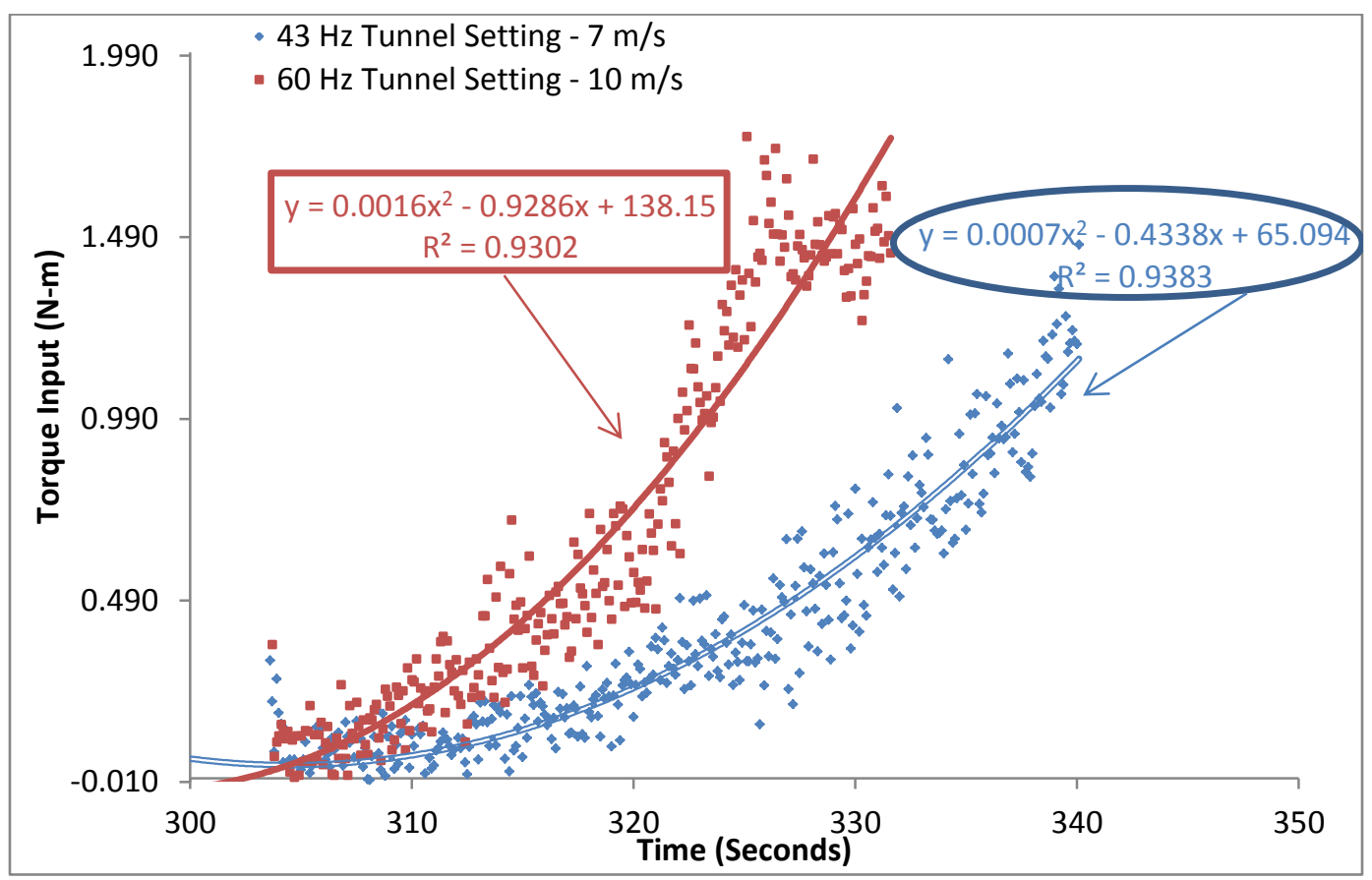

Figure 89. Full-Scale Ramp-Up Torque Comparison with Respect to Time

\section{Computational Results: Blade Element Analysis}

In order to more accurately simulate and predict the effects of circulation control on this particular propeller geometry, a blade element analysis was done on both the baseline propeller and the circulation control augmented propeller used in this experimentation. Using both historical data (Appendix H - Two-Dimensional Clark-Y Airfoil Data) and computational estimations of sectional lift and drag profiles (Appendix G - Clark-Y Simulated Airfoil Data) for both propeller geometries, trend studies were conducted to provide insight into how the propeller performed when loaded and circulation control plenums were applied.

One major factor made completing this study inherently difficult. Because of the cost constraints of this experimental program, there was no way to make a model large enough to design a pressure sensor to monitor the outlet pressure of the circulation control plenum while in use. Steps were taken to statically measure the output plenum pressures and correlate these 
values to known usable forward velocities. Also taken into consideration was the rotational speed of the propeller as it would provide the pumping forces needed to pressurize the $C C$ plenum. These values were measured on a bench top apparatus with a known pressure input into the inlet of the plenum. The values measured where then used to estimate the resultant jet velocity $\left(V_{j}\right)$, and corresponding $C C$ coefficient $\left(C_{\mu}\right)$. Using the empirical relationship found in Equation 29, below, a change in theoretical lift coefficient was estimated due to the addition of $C C$ exit slots across a portion of the radius of the propeller (Loth, 1984).

$$
\Delta C_{l}=40\left(\frac{h}{c}\right)^{0.64}\left(\frac{V_{j}}{V_{\infty}}-1\right)
$$

Similar predictions were computed in the change in drag coefficient due to the rounded trailing edge of the airfoil in comparison to the theoretical values found for the baseline Clark-Y airfoil. XFLR-5, as well as another program called DesignFoil were both software packages that were employed to study the differences between the predicted aerodynamic forces experienced by a Clark-Y airfoil (un-augmented propeller) and the rounded trailing edge modification airfoil (augmented propeller) over a range of Reynolds Number inputs and angle-of-attack values. It is programmed to use the panel method to estimate the aerodynamic forces experienced by a particular airfoil shape when specifying Reynolds Number and angle-of-attack values.

As shown, in Figure 90 and Figure 91, the effect of Reynolds Number increase over the entire performance of the airfoil is not predicted to have a great impact on the section lift and drag coefficients of an un-augmented Clark-Y airfoil section. As assumed, the angle-of-attack at which stall is predicted to occur gradually increased as the Reynolds Number increased, however, it was no more than 5 degrees total over the maximum range of the study. Specific Reynolds Number analyses were chosen to estimate the force coefficients data at selected tunnel speed which were used during the experimental phase. This upper and lower bound study provided 
fixed coefficient data for the un-augmented propeller cross-section as well as a data bank for completing the blade element analysis portion of this study. Reynolds Number values of 500,000 was chosen as an upper limit for this study, and was included in the data acquisition to analyze the increase in trend of the propeller blade performance. This Reynolds Number was never reached during physical testing due to tunnel velocity limitations.

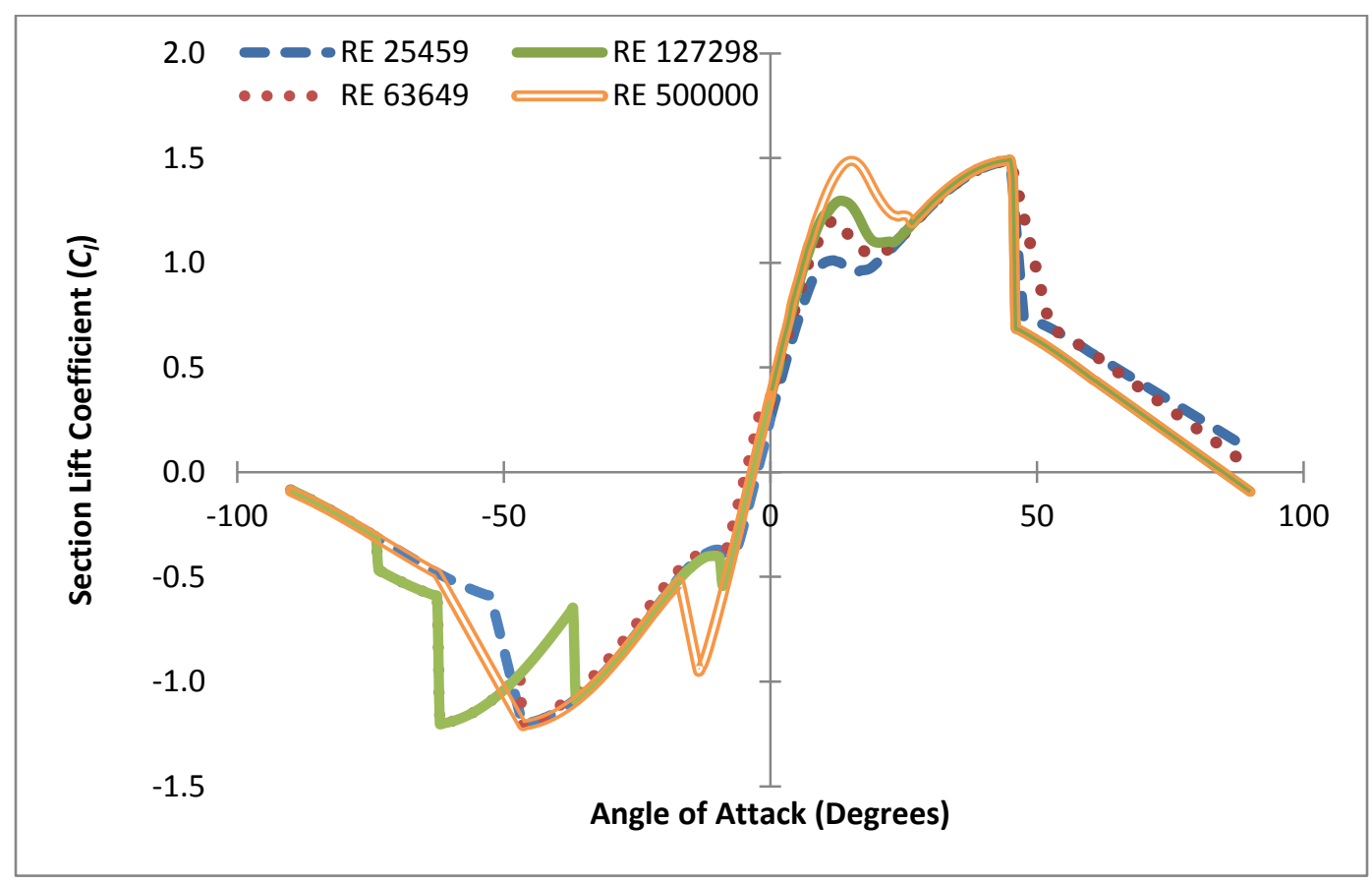

Figure 90. Gathered Lift Coefficient Data on Unaugmented Clark-Y Two-Dimensional Airfoil 


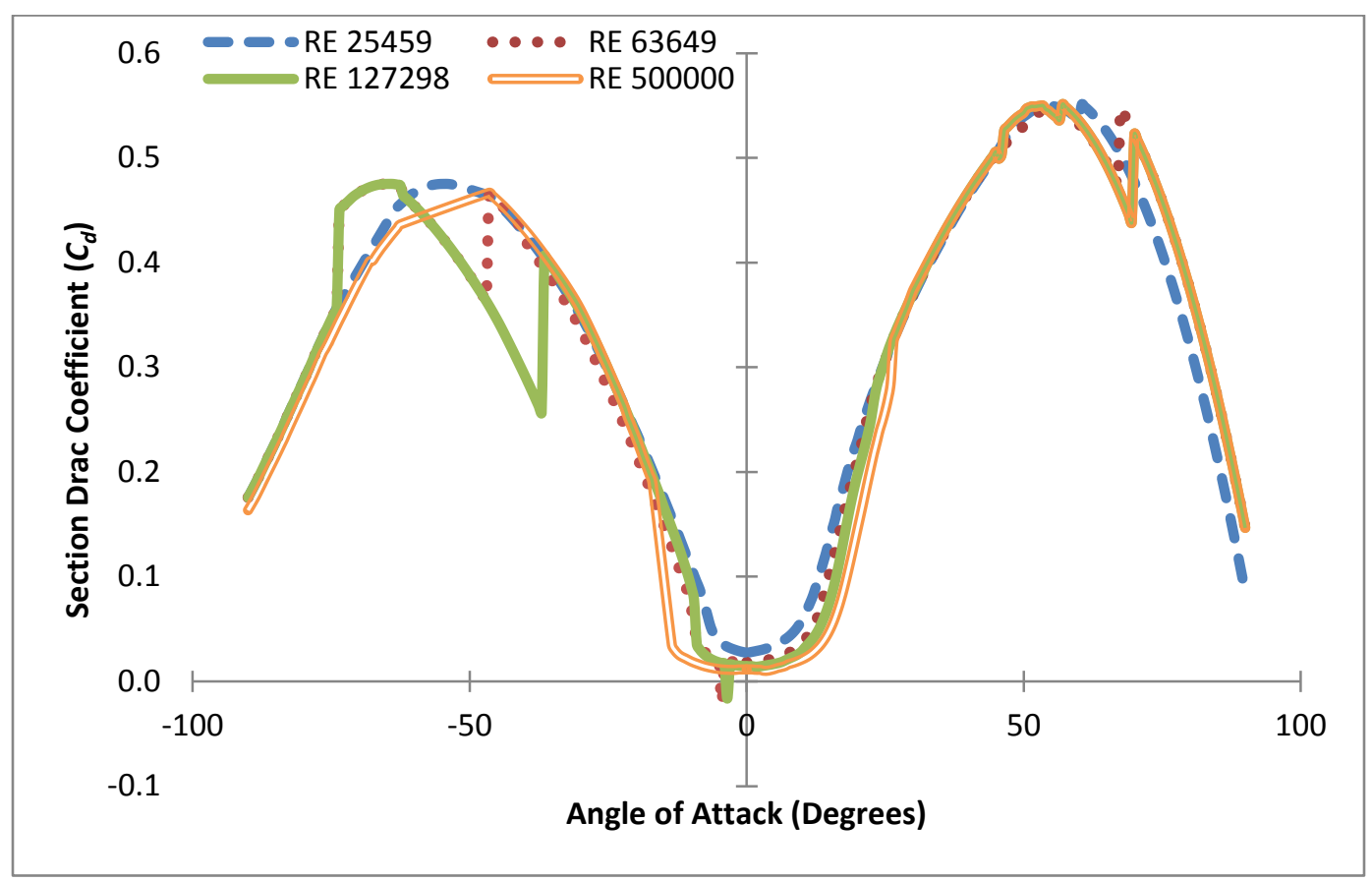

Figure 91. Unaugmented Two-Dimensional Drag Coefficient Data for Clark-Y Airfoil

In both of these plots, the positive and negative angles-of-attack are predicted, simply in order to show that the airfoil geometry is not symmetric, and because for certain types of flight regimes the propeller may see a negative angle-of-attack component. The trends of both the sectional lift coefficient and the sectional drag coefficient can be assumed to repeat after 90.0 and negative 90.0 degrees providing a full spectrum of 0 to 360 degrees angle-of-attack data for use in analysis of any flight regime.

Because the trailing edge slot was only applied to the radial locations between 33 and 66 percent of the entire radius, only these values of $C_{l}$ and $C_{d}$ were predicted to change. In order to simulate a trend study for this analysis three Reynolds Numbers (which corresponded to a particular tunnel speed or forward velocity) and three rotational speeds $(1000,2000$, and 3870 $\mathrm{rpm}$ ) were analyzed. This produced a trend study over the values of advance ratio that were most used during experimentation, 0.0 to 1.0 . 
In the following figures, Figure 92, Figure 93, and Figure 94 results of a blade element model are given with respect to both the unaugmented propeller, and the augmented propeller. First in Figure 92, the overall thrust output predicted shows an increase due to the addition of a circulation control exit jet on the training edge of the propeller blade. This increase seems to have the most effectiveness at advance ratios above 0.25 . This agrees with the experimental results in that the $C C$ propeller was better equipped to produce thrust forces above an advance ratio of 0.30 .

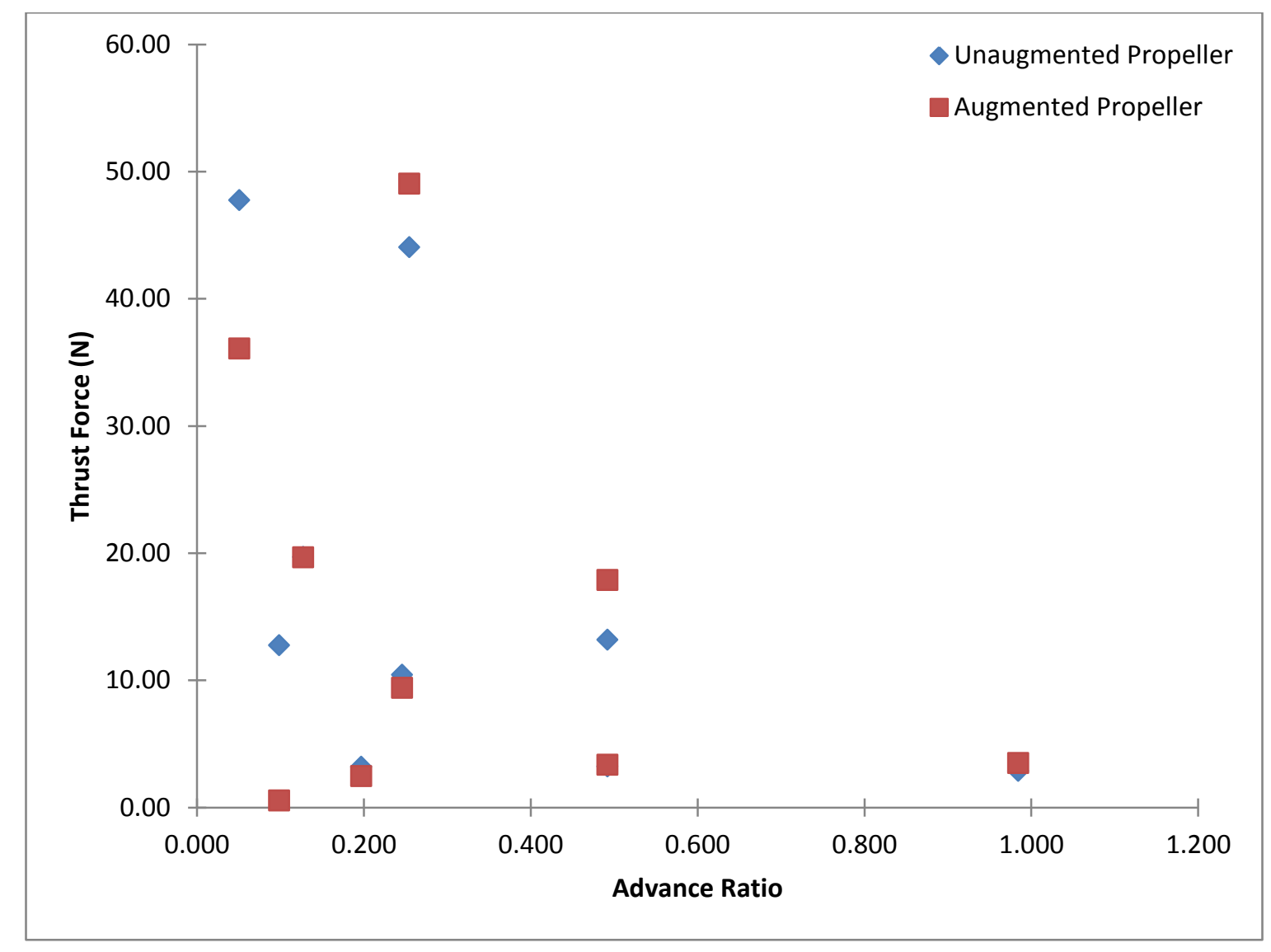

Figure 92. Thrust Force Analysis of Unaugmented and Augmented Propellers

A similar data reduction of the required torque needed to turn the propeller shows an increase in the unaugmented propeller case over the analyzed advance ratios, which is also consistent with the previous experimental results shown from wind tunnel testing. As the 
rotational speeds of the propeller increase, so too does the need for input torque to continue to drive the propeller, as is shown in Figure 93.

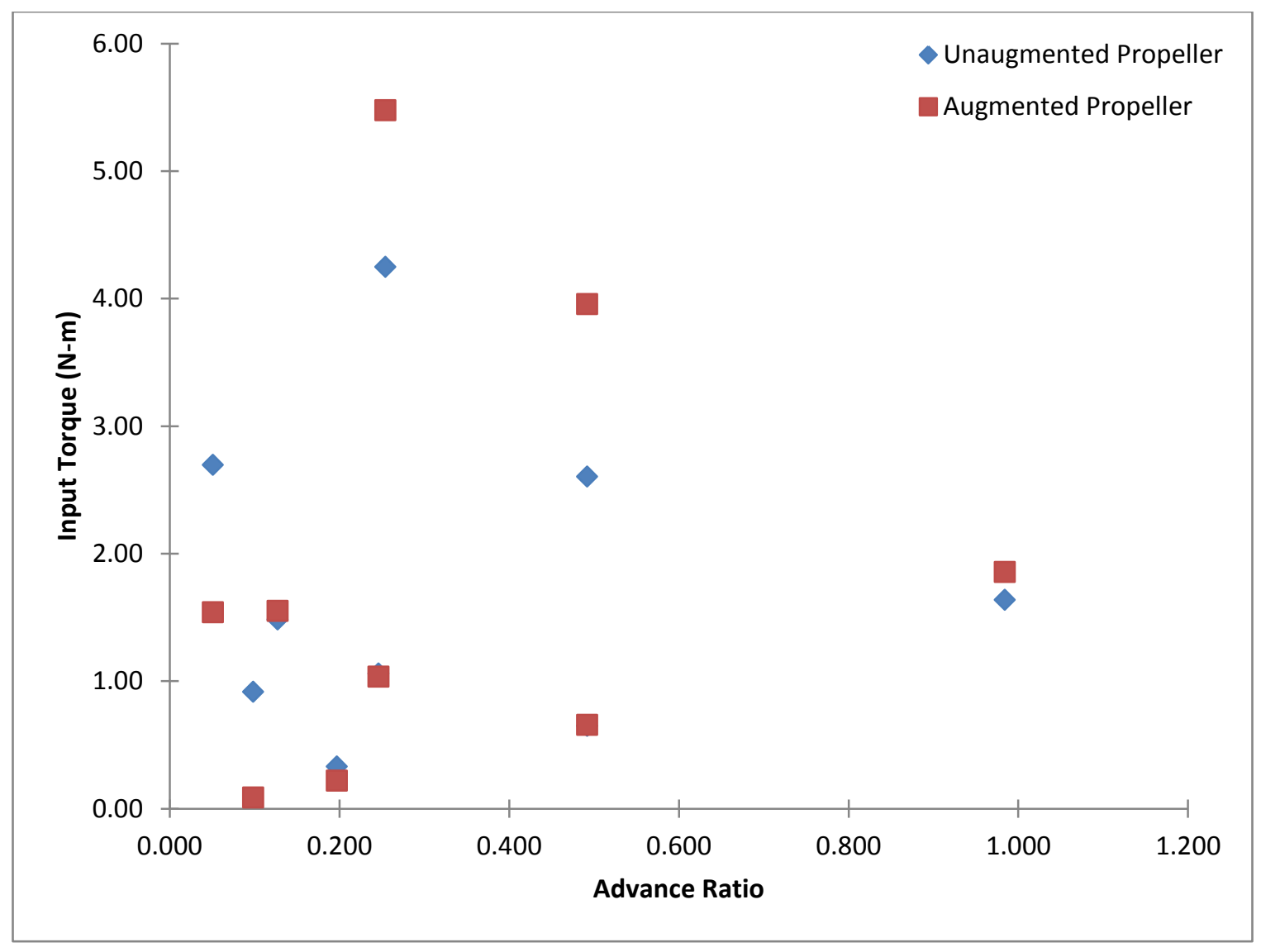

Figure 93. Input Torque Required for Unaugmented and Augmented Propellers

Finally, the overall efficiency of the propeller was calculated based on input and output force estimations from the computational results and the blade element modeling of the blade geometry. These results, similar to the experimental values show an increased efficiency of the unaugmented propeller at low advance ratios (less than 0.20) and an increased efficiency in the $C C$ augmented propeller at advance ratios above 0.20 . 


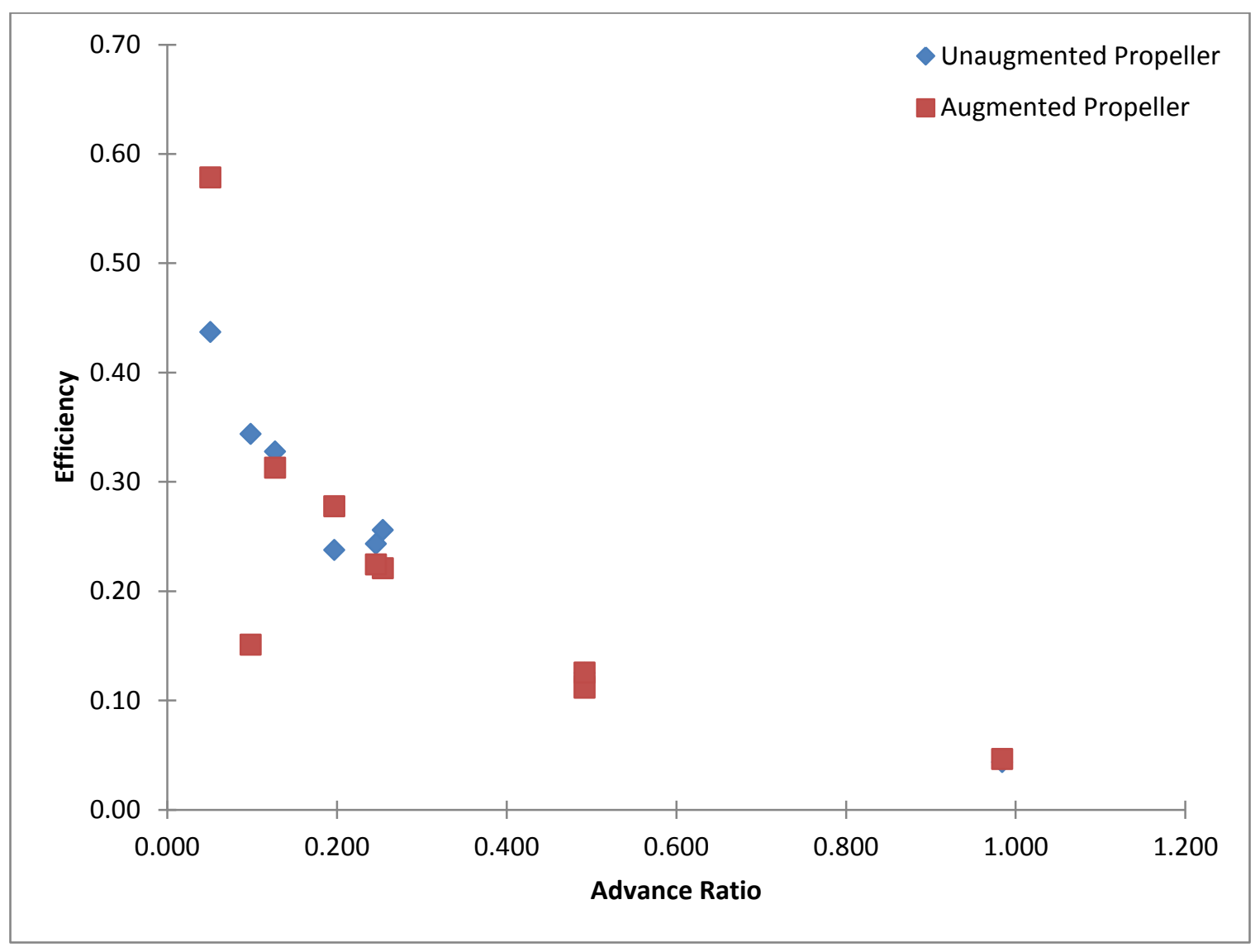

\section{Figure 94. Overall Efficiency of Propellers Analyzed through Blade Element Model}

This predictive type of programming approach and use of computer modeling techniques can be very powerful in ascertaining the performance of the propellers, both augmented and unaugmented before building any further models, just based on geometry and airflow characteristics.

Computational and experimental results were plotted together from both the $W V U$ experimental trials, and the $W P A F B$ testing scenarios to visually determine how accurate the blade element analysis is in comparison to the experimental results. In Figure 95, below, a maximum difference of 10 percent in final efficiency is shown to occur between the $W P A F B$ and $C F D$ cases that were run over specific $R e$ values. This difference in output is due to the inaccurate prediction of drag force in the computational modeling simulation software and can be taken into account in future simulations. 


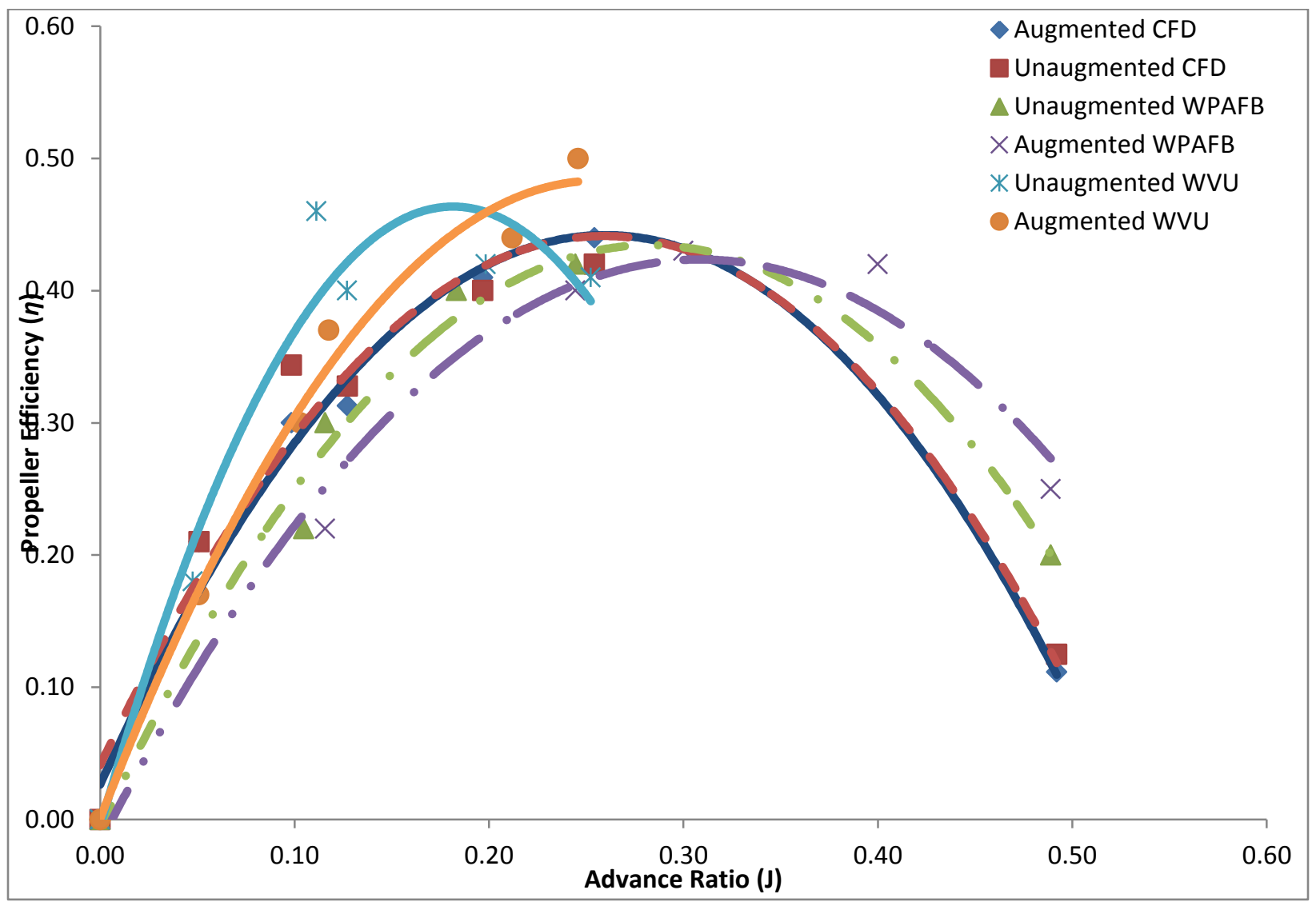

Figure 95. Experimental vs Computational Propeller Efficiency Results

\section{Experimental Error Analysis}

Error analyses and theoretical losses in the experimental equipment was performed. These error factors can be attributed to any number of operational conditions, or testing scenarios from the equipment chosen to the environment available for testing.

One main factor in the error reporting of this experiment was the electric motor used in supplying the propeller with mechanical power. Due to the fact that it was a brushless, outrunner type motor, meaning that the can of the motor spun on the outside of the copper coils instead of the inside of the coils, the motor itself was much more efficient than older electric models. This allowed for a wide spectrum of testing to be completed without the need to account for major losses in the experiment due to the motor overheating or friction becoming a problem. Below, in Figure 96, for the input voltage from the battery supply of the experiment it is shown that at the 
maximum operating rotational speed the efficiency factors of the Rimfire motor are said to be 88.0-89.0 percent efficient while only estimated to use 5.0-10.0 amps of input current. Also estimated was the current input and power usage of the motor during operational testing. Below, as the linear trend of power draw increased, as does the thermal power wasted to the motor coils. This was minimized in this experiment because, the rotational speed of the motor never increased above $4000 \mathrm{rpm}$.

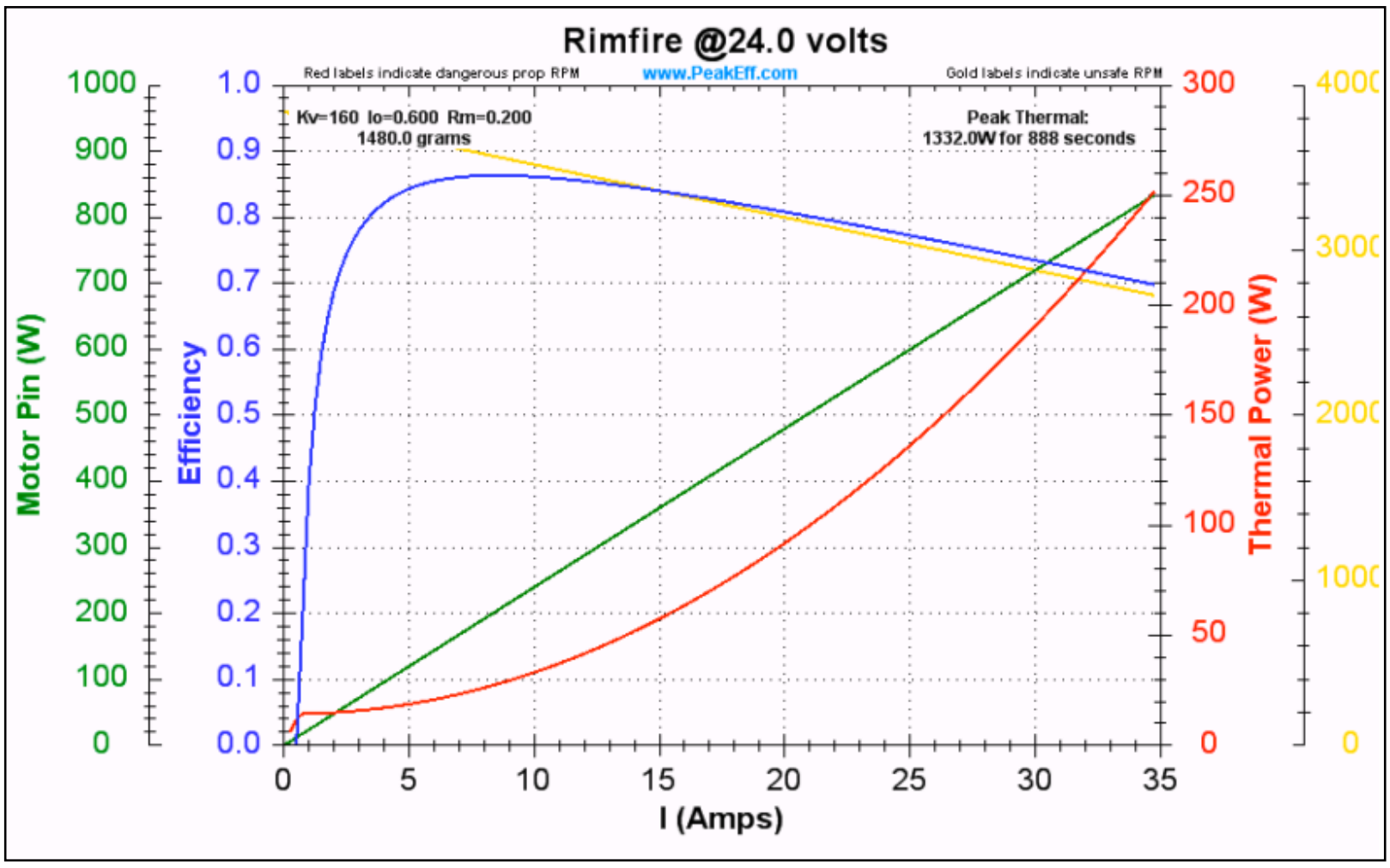

Figure 96. Manufacturer Performance Factors of the Rimfire Electric Motor (http://www.peakeff.com)

An error analysis of the hardware and measurement systems used in testing was done estimating the overall impact of the experimental components including the electric motor, speed controller, tunnel velocity measurements, load cell, and force balance readings, and input voltage and current values. Because of the two different experimental designs and the fact that there were 
different components in each of the tests, it was necessary to estimate a separate error value for both experiments.

In the experiments conducted at $W V U$, as outlined in previous sections, the measurements taken included a temperature of the motor coils, a real-time voltage drop across the shunt installed in-line with the battery power supply, a velocity reading from an ultrasonic anemometer, and the load cell value of the force applied to the test stand by the propeller.

Alternatively, the experiments conducted at WPAFB included a data acquisition system which tabulated data from an array of sensors including an ultrasonic anemometer, two pressure transducers, a Hall Effect Sensor to measure rotational speed, and a six component force balance, which measured axial forces and moments due to propeller loading (translated to thrust and torque required). The Hall Effect sensor was mounted directly to the test stand and was matched with a rare-earth magnet which was then used to measure the rotational speed capabilities of the propeller while testing. This sensor was then monitored via a counter which a voltage change every time the propeller magnet moved past the sensor.

In the following figures, Figure 97 and Figure 98, the overall efficiency rating of the two propellers is plotted against the experimental advance ratio. It is shown in Figure 97 that the experimental values measured while testing with the unaugmented propeller, from both wind tunnel experiments, show small variance between one another despite the differing hardware used in measuring during experimentation for low values of advance ratios. As the advance ratios approach and surpass 0.30 , however, the $W V U$ experiments seem to become less accurate as the limits of the testing equipment started to become influential in the experimental results. Similar data is seen in Figure 98 when analysis of the augmented propeller was completed. The trends between the two experiments tend to vary little when the advance ratio is less than 0.3 , but as the value of advance ratio continues to climb, the limits of the experimental equipment again 
seem to impact the overall output data. Maximum efficiency error of the unaugmented propeller is found at 0.43 advance ratio and has an estimated value of 10.0 percent. In the case of the augmented propeller the difference in efficiency is shown maximum at a value of 0.23 advance ratio and has a difference between experimental results of 14.3 percent.

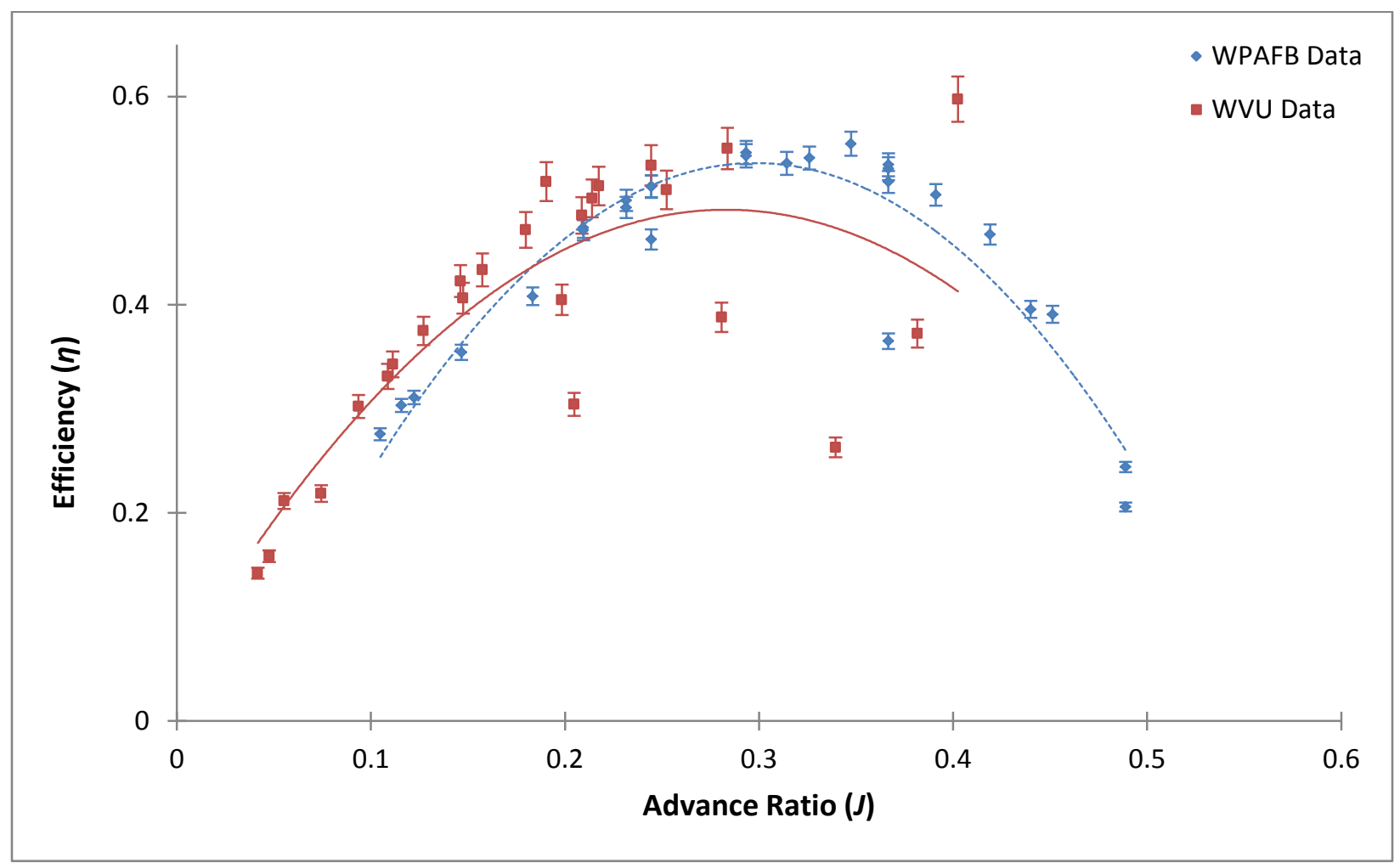

Figure 97. Unaugmented Propeller Efficiency Data Collection Error Analysis 


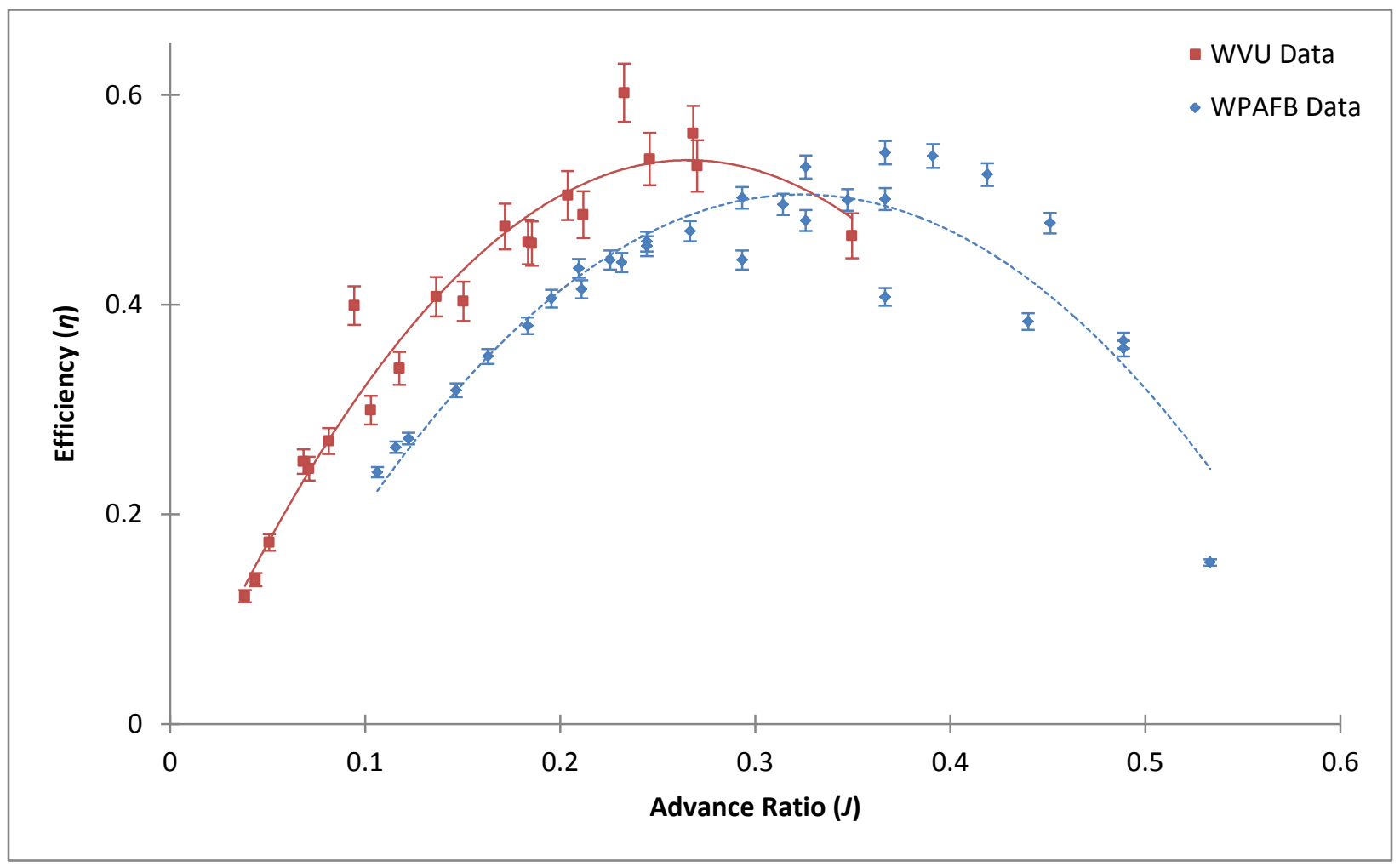

Figure 98. Augmented Propeller Efficiency Data Error Analysis

A statistical survey of the experimental data was done in order to ascertain the goodness of fit, and the reliability of the final data throughout the experimental examination. Using previous work done in describing experimental uncertainty in experimental measurements, it was possible to calculate a similar factor for these experiments. According to a publication from 1988, the uncertainty of a value is based on Equation 30, below (Moffat, 1988)

$$
\partial R=\left\{\sum_{i=1}^{N}\left(\frac{\partial R}{\partial X_{i}} \partial X_{i}\right)^{2}\right\}^{.5}
$$

Equation 30

This equation basically states that the uncertainty of any experimental variable (R) is based on the sum of the squares of partial derivatives with respect to each of the measured terms $\left(\mathrm{X}_{\mathrm{i}}\right)$. Shown in Figure 99, for these specific experiments the efficiency of the propeller was the main result of the study and was based on the measure input torque and the measure output thrust. Looking at the final values for each of the experiments, and defining the value of the 
standard deviation and statistical relevance of the data, it is shown that at advance ratios from 0.0-0.3, the resulting data and standard deviations actually overlap one another from both experiments. This lends itself to the idea that the final results of the propeller test show little to no improvement to the performance at low advance ratios. However, as the advance ratio of the propeller increases above 0.3 during testing the augmented propeller begins to show a greater improvement over the unaugmented propeller, with the confidence intervals to back up the data. Overall, the experiments at $W V U$ showed an uncertainty of 1.6 percent (augmented cases) and 1.1 percent in the unaugmented cases. Similarly, when the results from the $W P A F B$ tests were analyzed, the augmented propeller tests showed a 1.4 percent uncertainty, compared to the 1.2 percent uncertainty in the unaugmented cases. Generally during both experimental runs, the unaugmented case showed a better percent uncertainty in comparison to the augmented propeller.

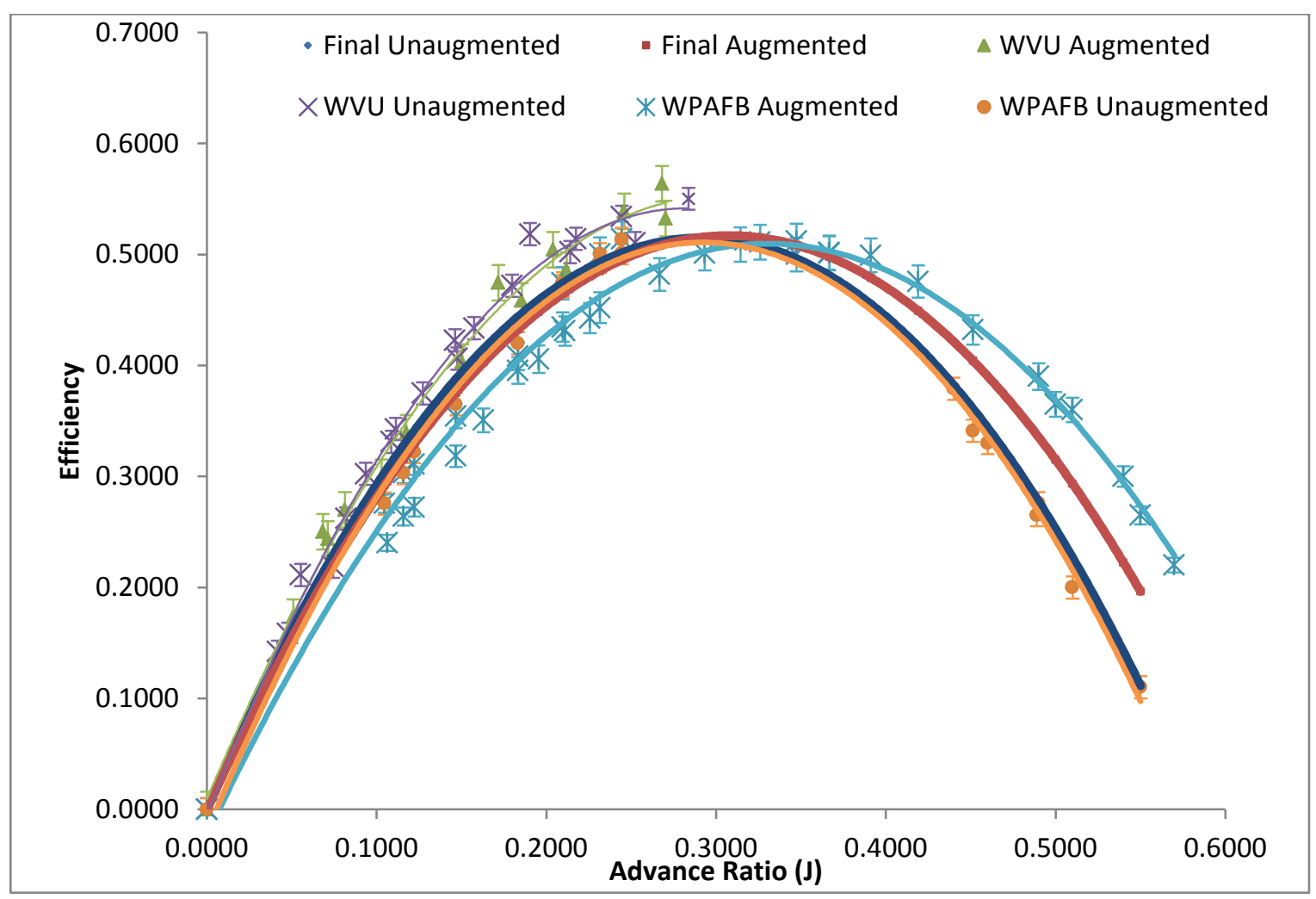

Figure 99. Statistical Analysis of Gathered Experimntal Data 


\section{Conclusions}

It is clear that this design requires a systems approach for utilization and will entail the idea of redesigning of the propeller blade and its application. Numerous studies have been conducted in the past on propellers including the use of active blowing slots to create large increases in thrust delivery. As with most active circulation control systems, use of the hardware, weight, and pumping penalties far outweigh the benefits for all but few unique applications. In contrast, and particularly regarding consumption of fossil fuel and environmental consciousness, any improvements, even single digit improvements will provide value to the $U A V$ industry. It is shown that this technology can provide such a benefit. With the employment of a retro-fitted passively pressurized circulation control propeller, the need for changing the existing hardware vital to the aircraft's design can be avoided. The use of this passive circulation control system avoids the need for excess weight additions, such as pumping systems, to control the augmented jet velocity.

The preliminary study of the circulation control propeller at changing input rotational speeds provided an accurate measurement of required torque throughout the ramping scenarios, however, the forces due to thrust were not as accurate to predict. This was possibly due to the response time of the load cell in comparison to the sampling rate of the experiment, and the application of human interface error on turning a dial to increase or reduce the rotational speed of the propeller. Another facet of this particular portion of the experiment was the inclusion of some free-stream anomalies at maximum tunnel velocity. Steady, exact flow conditions are needed to accurately log the forces of thrust in a dynamic response experiment. Further tunnel enhancements including lengthening the tunnel or adding straighteners and honeycomb screens could have proved beneficial during this portion of the experiment. Overall, this particular 
testing scenario was conducted to initialize the idea of dynamic range of this particular prototype and was secondary to proving proof-of-concept.

Also shown through this study was that the testing methods of experimentation of the two propellers in two different wind tunnels had little effect on the resultant data. The $W V U$ wind tunnel and experimental equipment performed well at low advance ratios (values $0.0-0.3)$ and did not begin to show experimental bias until an advance ratio of 0.35 . Conversely, the instrumentation at the vertical wind tunnel, at $W P A F B$, was able to provide accurate measurement data for experimental runs above 0.35 advance ratio for the same experimental propellers. Essentially, this was due to the limited amount of resources available to the graduate program at $W V U$, which resulted in the use of second-hand materials, components, and data acquisition devices during experimentation to save on construction costs. When experimenting in the vertical tunnel in Ohio, the already existing hardware and testing apparatuses located at $W P A F B$ have been fine-tuned and programmed over many years of use, and thus had already maximized their capabilities to provide reliable, repeatable results. The use of the $C C$ propeller showed that a 5.74 percent increase in aerodynamic efficiency was achievable when replacing the existing propeller with the designed $C C$ propeller.

This research showed that through previous literature studies, computational analysis, and wind tunnel experimentation, the passively pressurized system applied to a propeller can provide a net increase in overall efficiency of the aircraft, and thus provided proof-of-concept for the application of the technology. This concept, currently envisioned for unmanned aerial vehicle $(U A V)$ applications, suggests a single digit percent increase in the overall flight range of the aircraft. Although small by conventional circulation control application standards, this improvement could greatly increase the overall versatility of the aircraft and is particularly 
attractive because there is no need for additional hardware additions to the currently used aircraft. It merely requires a retro-fitted propeller to the already existing aircraft frame. 


\section{Recommendations and Future Research Considerations}

This research work specifically looked into the application of adding a passively controlled circulation plenum and ejection slot to improve overall range performance in $U A V$ application. It stands to reason, through scaling techniques and Reynolds Number matching, that the application of this technology to larger propeller driven aircraft would behave similarly, but future testing scenarios to prove this hypothesis will need to be scheduled. Further work into the rotational aspect of the plenum pumping forces and experimentation into tailoring the plenum to each application is crucial.

Since the forward flight velocities and rotational forces acting on the propeller are the main driving forces for the pressurized air in the $C C$ plenum, this design process of the inlet capture area should be optimized. Inlet veins and preventative measures for air rejection as well as optimization of plenum pressurization flow characteristics should also be taken into account. The aerodynamic design of the interior system has the potential to provide a similar benefit to the overall performance of the propeller as the rotational forces and pumping pressure meaning that a $C F D$ study into the shape and sizes of the interior plenum may provide the opportunity to increase the efficiency of the system.

In addition to optimization of the pressurization plenum, the exit slot and inlet to the pressurization chamber should also be optimized based on the applications. The selection of the position along the radius and the jet exit dimensions will allow for optimization of the entire system, as well as making the exit area the limiting dimension of the system to provide consistent pressurization for the jet velocity. Both of these facets of the design are mainly dependent upon how the propeller is manufactured, and the smaller the model becomes, the harder it is to accommodate resilient materials in construction. In order to be able to study this effect a particle image velocimetry $(P I V)$ wind tunnel study could be helpful in determining the 
actual output velocities and pressures of the circulation control plenum directly at the exit slot. Without a built-in way of measuring the output pressure of the plenum, the rotational aspect of the system makes data collecting difficult. Due to cost constraints of this particular experiment, no such study was feasible. Future experimental model considerations could possibly include a pre-existing mounted hotwire anemometer or ultrasonic anemometer near and/or at the inlet and exit of the $C C$ plenum in order to accurately determine the input and output velocities available during testing. Velocity and pressurization gages could also be useful within the plenum itself in order to read the maximum and minimum values which the plenum can be pressurized during use. Due to the rotational nature of this experimental set-up, these electronics used to measure velocities and pressures within the experimental system would also need to be able to transmit data wirelessly, or through a slip ring to avoid interference with the propeller.

Also, a possible area of interest is the higher rotational speed testing regimes of a propeller model. As this study was meant to show how the technology behaved, the high end speeds and subsequent higher tip Reynolds Numbers, and fully turbulent flow characteristics could show interesting effects when transferred through the ranges of incompressible and compressible fluid behavior as well as sonic and trans-sonic speed regimes. Again, the use of stronger modeling materials and testing equipment would need to be used in order to study these effects. Perhaps a larger scale testing scenario would also be beneficial for this type of experimentation. West Virginia University is already equipped to deal with such an experiment by retro-fitting a large scale model to the Facility for Outdoor Rotor Testing at the WVU Hangar.

As it was discussed earlier in this research, compressible fluid (i.e. water, natural gasses, etc.) applications are also feasible. The compressibility effects of these fluids and natural occurrences of cavitation along the blade diameter could prove to show interesting effects with the use of the $C C$ slot. It is recommended that future considerations be taken into studying these 
effects in a compressible environment. Using a fluid such as water or natural gas would alleviate the need for testing this system in an air environment at high speeds to simulate compressibility effects.

Future research into providing the forward velocity pumping forces should be considered on a per-application basis. The rerouting of stagnation pressures from the system's use on a rear drive "pusher" type propeller would be vastly different from having the stagnation forces directly at the propeller hub of a forward drive "puller" type craft. The application experimentations would be similar to the use of the technology on aircraft and the overall system would need to be optimized based on the specific use.

Another potential application of this technology can be used for alternative rotorcraft, and rotational bodies (i.e. helicopters, wind turbines, flywheels). The use of this type of system has the potential to provide for an interesting experiment, with lower free-stream velocities to pump air into the chambers for pressurization of the $C C$ plenum, and alternative rerouting of the stagnation pressures, the system would need to rely more heavily on the rotational forces produced while spinning to gather the pressure required in the $C C$ chamber. Also changing the dynamic of the system is the oscillation of the blade pitch during rotation by the swash plate or similar device. While the rotating body is turning it is constantly being made to find the best angle-of-attack to the forward wind velocity to greater enhance the performance, which will affect the overall performance of the $C C$ system if it is applied to one of these systems.

A final area of interest into the future studies of this technology is to see what effects the slow and fast altitude variance (nominal climb and descent and/or dog-fighting) as well as, fast variation of rotational speeds have on the overall performance of the circulation control system. With the change in altitude come several parameter changes which will need to be analyzed, such as temperature, pressure, density, and moisture level changes, all can have an effect on this 
system's ability to function properly. Similarly, while in conditions suited for fast maneuvering, the angle the propeller meets the free-stream air as well as density and pressure changes across altitudes may also pose a severe impact on the system. The passive nature of the system allows for it to be always on or always off, however if there are deteriorating benefits while the craft is in cruise, then it may be beneficial to only use this type of system when it is truly needed, specifically on start-up (take-off) and shut-down (landing) adding a shut-off valve consideration to the overall design of the inlet system. 


\section{References}

1. Abbott, I.H., Von Doenhoff, A. E., Theory of Wing Sections. Dover Publications, Inc, New York, NY, 1959.

2. Abramson, J., "Two-Dimensional Subsonic Wind Tunnel Evaluation of a 20-Percent-Thick Circulation Controlled Airfoil.” David W. Taylor Naval Ship Research Center, Bathesda, MD, 1975.

3. Abramson, J., "Characteristics of a Cambered Circulation Control Airfoil Having Both Upper and Lower Surface Trailing Edge Slots.” Naval Surface Warfare Center - Carderock Division, Bathesda, MD, 2004.

4. Ambrosiani, J.P., "Analysis of a Circulation Controlled Elliptical Airfoil.” Ph.D. Dissertation, Department of Mechanical and Aerospace Engineering, West Virginia University, Morgantown, WV, 1971.

5. Anderson Jr., J.D., Fundamentals of Aerodynamics. McGraw Hill, Inc., New York, NY, 2001.

6. Angle II, G.M., “Aerodynamic Benefits of Near-Surface-Actuated Circulation Control Blowing Slots for Rotorcraft Use." Ph.D. Dissertation, Department of Aerospace Engineering, West Virginia University, Morgantown, WV, 2008.

7. Angle II, G.M., Huebsch, W.W., Prucz, J.C., Smith, J.E., "Circulation Controlled, FixedRoot Helicopter Concept." American Helicopter Society 62nd Annual Forum, Phoenix, AZ, 2006.

8. Aria Wind Power Systems Inc., University of Victoria, "Water Tunnel Propeller Dynamometer." http://design.engr.uvic.ca/student-projects/engr400/projects/aria2/ Accessed: October, 21, 2009.

9. Ash, R.L., Britcher, C.P., Hyde, K.W., "prop-Wrights: How Two Brothers from Dayton Added a New Twist to Airplane Propulsion." Mechanical Engineering - 100 Years of Flight. December, 2003.

10. Ashworth, J.C., "An Experiment Investigation of a Circulation Controlled Shrouded Propeller." Masters Thesis, Department of Mechanical and Aerospace Engineering, West Virginia University, Morgantown, WV, 19773. 
11. Bedell, F., The Air Propeller. Carveth Press, New York, NY, 2008.

12. Bertin, J.J., Aerodynamics for Engineers. Prentice-Hall, Inc., Upper Saddle River, NJ, 2002.

13. Braslow, A.L., "Aerodynamic Evaluation of Circulation Control Propellers.” Contractor Report \#165748, National Aeronautics and Space Administration (NASA) and The Bionetics Corporation, Hampton, VA, 1981.

14. Carlton, J., Marine Propellers and Propulsion, Elsevier Science, Cambridge, MA, 2007.

15. Churchill, R.A., “Coandă Effect Jet around a Cylinder with an Interacting Adjacent Surface.” Ph.D. Dissertation, Department of Aerospace Engineering, West Virginia University, Morgantown, WV, 1992.

16. Delano, J.B., "Investigation of the NACA 4-(5)(08)-03 and NACA 4-(5)(10)-03 Two-Bladed Propellers at Forward Mach Numbers to 0.725 to Determine the Effects of the Camber and Compressibility on Performance.” National Advisory Committee for Aeronautics, Report No. 1012. Langley Field, VA. 1945.

17. Dommasch, D.O., Elements of Propeller and Helicopter Aerodynamics. Pitman Publishing Corporation, New York, New York, 1953.

18. Dommasch, D.O., Sherby, S.S., Connolly, T.F., Airplane Aerodynamics. Pitman Publishing Corporation, New York, New York, 1961.

19. Englar, R.J., “Two-Dimensional Subsonic Wind Tunnel Tests of Two 15-Percent Thick Circulation Control Airfoils,” David W. Taylor Naval Ship Research and Development Center Technical Note AL-211, Washington, DC, 1971.

20. Englar, R.J., Williams, R.M., "Design of a Circulation Control Stern Plane for Submarine Applications.” Naval Ship Research and Development Center, Bethesda, MD, 1971.

21. Englar, R.J., Williams, R.M., “Test Techniques for High-lift, Two-Dimensional Airfoils with Boundary Layer and Circulation Control for Application to Rotary-wing Aircraft." Canadian Aeronautics and Space Journal, Vol. 19, No. 3, 1973.

22. Englar, R.J., "Subsonic Two-Dimensional Wind Tunnel Investigation of the High-lift Capability of Circulation Control Wing Sections.” David W. Taylor Naval Ship Research and Development Center, Bathesda, MD, 1975. 
23. Englar, R.J., "Experimental Investigation of the High Velocity Coandă Wall Jet Applied to Bluff Trailing Edge Circulation Control Airfoils.” David W. Taylor Naval Research and Development Center, Report \#4708, September, 1975.

24. Englar, R.J., "Development of the A-6/Circulation Control Wing Flight Demonstrator Configuration.” David W. Taylor Naval Ship Research and Development Center, Bathesda, MD, 1979.

25. Englar, R.J., Nichols, J.H., Harris, M.J., Eppel, J.C., Shovlin, M.D., “Circulation Control Technology Applied to Propulsive High-lift Systems." Society of Automotive Engineers, Aerospace Congress and Exposition, Long Beach, CA, 1984.

26. Englar, R.J., "The Application of Circulation control Pneumatic Technology to Powered-lift STOL Aircraft.” Advanced Flight Systems Department, Lockheed Aeronautical Systems Company, Marietta, GA, 1987.

27. Englar, R.J., Smith, M.J., Neibur, C.S., Gregory, S.D., "Development of Pneumatic Aerodynamic Concepts for Control of Lift, Drag and Moment plus Lateral/Directional Stability of Automotive Vehicles.” Georgia Tech Research Institute, Journal of Technology, Atlanta, GA, 1996.

28. Englar, R.J., "Overview of Circulation Control Pneumatic Aerodynamics: Blown force and Moment Augmentation and Modification as Applied Primarily to Fixed-wing Aircraft." NASA/ONR Circulation Control Workshop, National Aeronautics and Space Administration, CP-2005-213509, 2005.

29. Englar, R.J., "Pneumatic Aerodynamic Technology to Improve Performance of Automotive Vehicles.” American Institute of Aeronautics and Astronautics, 2005.

30. Englar, R.J., "The Application of Pneumatic Aerodynamic Technology to Improve Performance and Control of Advanced Automotive Vehicles.” Georgia Tech Research Institute: Aerospace, Transportation, and Advanced Systems Lab, Atlanta, GA, 2009.

31. Ericsson, J., “Ship Propeller.” U.S. Patent 4181, 1845.

32. Franke, M.E., Harvell, J.K., "Wind Tunnel Studies of Circulation Control Elliptical Airfoils.” Air Force Institute of Technology, Tech \#N88-17598, Wright-Patterson Air Force Base, Dayton, OH, 1987. 
33. Gibbs, E.H., “Analysis of Circulation Controlled Airfoils.” Ph.D. Dissertation, Department of Mechanical and Aerospace Engineering, West Virginia University, Morgantown, WV, 1975.

34. Glauert, H., The Elements of the Aerofoil and Airscrew Theory. Cambridge University Press, New York, NY, 1943.

35. Hansen, J. "Fortus 900mc Accuracy Study.” Fortus 3D Production Systems, Stratasys, Inc. http://www.fortus.com/Products/ /media/Fortus/Files/PDFs/WPFortus900mcAccuracyStudy.ashx Accessed: August 24, 2009.

36. Harness, G.S., “An Experimental Investigation of a Circulation Controlled Cambered Elliptical Airfoil.” Masters Thesis, Department of Aerospace Engineering, West Virginia University, Morgantown, WV, 1970.

37. Holt, D.J., "Circulation Controlled Airfoil with Pulsed Flow." Masters Thesis Department of Mechanical and Aerospace Engineering, West Virginia University, Morgantown, WV, 1972.

38. Katz, J., Plotkin, A., Low Speed Aerodynamics. Cambridge University Press, Cambridge, NY, 2001.

39. Kind, R.J., Maul, D.J., “An Experimental Investigation of a Low-Speed CirculationControlled Aerofoil.” The Aeronautical Quarterly, Vol. XIX, May, 1968.

40. Kueth, A.M., Schetzer, J.D., Foundations of Aerodynamics. John Wiley and Sons Inc., New York, NY, 1967.

41. Kweder, J., Clarke, M., Smith, J.E., "Investigation into the Feasibility of an Augmented Propeller Design with the use of a Passive Circulation Control System," American Society of Mechanical Engineers (ASME), 2010 International Mechanical Engineering Congress \& Exposition. Vancouver, British Columbia, Canada. November 2010.

42. Kweder, J., Panther, C.C., Smith, J.E., “Applications of Circulation Control, Yesterday and Today," International Journal of Engineering (IJE). Volume 4, Issue 5. CSC Press, Kuala Lumpur, Malaysia, October 2010. 
43. Loth, J.L., Fanucci, J.B., Roberts, S.C., "Flight Performance of a Circulation Controlled STOL Aircraft." American Institute of Aeronautics and Astronautics, Journal of Aircraft, Vol. 13, No. 3, 1976.

44. Loth, J.L., Boasson, M., “Circulation Control STOL Wing Optimization.” American Institute of Aeronautics and Astronautics, Journal of Aircraft, Vol. 21 No. 2, February, 1984.

45. Loth, J.L., Funk, M.S., "Thrust Savings Limitations with Blown high-lift Wings.” American Institute of Aeronautics and Astronautics, AIAA/AHS/ASEE Aircraft Design, Systems and Operations Meeting, St. Louis, MO, 1987.

46. Marchman III, J.F., Werme, T.D., "Clark-Y Airfoil Performance at Low Reynolds Numbers." American Institute of Aeronautics and Astronautics, $22^{\text {nd }}$ AIAA Aerospace Sciences Meeting, Reno, NV, 1984.

47. Maskell, E.C., Spence D.A., “A Theory of the Jet Flap in Three Dimensions,” Royal Society of London, A Mathematical and Physical Sciences Series, Vol. 251, No. 1266, 1959.

48. Moffat, R.J., "Describing the Uncertainties in Experimental Results.” Experimental Thermal and Fluid Science, Elsevier Science Publishing Co., Inc., New York, NY, 1988.

49. Mosher, M., "Acoustics of Rotors Utilizing Circulation Control.” American Institute of Aeronautics and Astronautics, Journal of Aircraft, Vol. 20, No. 11, 1981.

50. Mills, A.F., Basic Heat and Mass Transfer. Prentice Hall, Inc. Upper Saddle River, NJ, 1999.

51. Myer, D.P., “An Investigation of a Circulation Controlled Cambered Elliptical Airfoil with a Rounded Trailing Edge.” Masters Thesis, Aerospace Engineering Department, West Virginia University, Morgantown, WV, 1972.

52. Naqvi, M.A., "Prediction of Circulation Control Performance Characteristics for Super STOL\& STOL Applications.” Ph.D. Dissertation, School of Aerospace Engineering, Georgia Institute of Technology, Atlanta, GA, 2006.

53. National Aerospace Laboratory (NRL), The Netherlands, "Propulsion Test Rigs", http://www2.nlr.nl/public/facilities/AVET-Info/Content/UK/Equip_PTR.html, Accessed October, 2009. 
54. Newman, B.G., "The Deflexion of Plane Jets by Adjacent Boundaries - Coandă Effect." Boundary Layer Flow Control, Pergamon Press, Vol. 1, 1961.

55. Nelson, W.C., Airplane Propeller Principles. John Wiley and Sons, Inc., London, England, 1944.

56. Novak, C.J., Cornelius, K.C., Roads, R.K., "Experimental Investigations of the Circular Wall Jet on a Circulation Control Airfoil." American Institute of Aeronautics and Astronautics, 25 ${ }^{\text {th }}$ Aerospace Sciences Meeting, Reno, NV, 1987.

57. Ottensoser, J., “Two-Dimensional Subsonic Evaluation of a 15-Percent Thick Circulation Control Airfoil with Slots at Leading and Trailing Edges.” Naval Ship Research and Development Center, Bethesda, MD, 1974.

58. Peak Efficiency Calculations, http://peakeff.com, Accessed: June 11, 2012.

59. Rae Jr., W.H., Shindo, S., "Comments on V/STOL Wind Tunnel Data at Low Forward Speeds.” Washington University, Seattle, WA, 1969.

60. Reader, K.R., Wilkerson, J.B., "Circulation Control Applied to a High Speed Helicopter Rotor.” David W. Taylor Naval Ship Research and Development Center, Bathesda, MD, 1976.

61. Reissner, H., Theory of Propellers: Advanced Instruction and Research in Mechanics. Brown University, Providence, RI, 1942.

62. Rogers, E.O., Donnelly, M.J., "Characteristics of a Dual-Slotted Circulation Control Wing of Low Aspect Ratio Intended for Naval Hydrodynamic Applications.” American Institute of Aeronautics and Astronautics, 42nd Aerospace Sciences Meeting, Reno, NV, 2004.

63. Seif, M.S, Tavakoli, M.T., "New Technologies for Reducing Fuel Consumption in Marine Vehicles.” XVI SORTA Symposium, Croatia, 2004.

64. Smith, F.P., “Screw Propeller.” US Pat. 2353, 1841.

65. Smith, J.E., Loth, J.L., Craven, R.P.M., Bond, R., "Piezoelectric Actuators for Circulation Controlled Rotorcraft.” US Patent 6,425,553 B1. 2002.

66. Stepniewski, W.Z., Kweys, C.N., Rotary-wing Aerodynamics. Dover Publications, Inc., New York, NY, 1981. 
67. Tai, J.C., Mavris, D.N., Schrage, D.P., “An Assessment of a Reaction Driven Stopped Rotor/Wing Using Circulation Control in Forward Flight.” American Institute of Aeronautics and Astronautics, World Aviation Congress, Los Angeles, CA, 1996.

68. Theodorsen, T., Theory of Propellers. McGraw-Hill Book Company, Inc. New York, NY, 1948.

69. Theodorsen, T., "Theory of Wing Sections of Arbitrary Shape.” National Advisory Committee for Aeronautics, NACA-TR-411, Langley Field, VA, 1931.

70. Thwaites, B., Incompressible Aerodynamics - An Account of the Theory and Observation of the Steady Flow of Incompressible Fluid past Aerofoils, Wings, and Other Bodies. Oxford University Press, New York, NY, 1960.

71. Tongchitpakdee, C., "Computational Studies of the Effects of Active and Passive Circulation Enhancement Concepts on Wind Turbine Performance." Doctoral Dissertation, School of Aerospace Engineering, Georgia Institute of Technology, Atlanta, GA,2007.

72. Vezzosi, A., Leonardo da Vinci: The Mind of a Renaissance. Harry N Abrams Inc., New York, NY, 1997.

73. Ward, C.C., "Circulation Control Technique on a Marine Propeller Duct." Masters Thesis, Department of Naval Architecture and Marine Engineering, University of Strathclyde, Glasgow, U.K., 2006.

74. White, F.M., Viscous Fluid Flow. McGraw-Hill, Inc., New York, NY, 2006.

75. Wilhelm, J.P., Pertl, E.D., Pertl, F.A., Smith, J.E., "Performance Predictions of a Circulation Controlled-Vertical Axis Wind Turbine with Solidity Control.” American Society of Mechanical Engineers, Energy Sustainability Conference, San Francisco, CA, 2009.

76. Wilhelm, J.P., Panther, C.C., Pertl, F.A., Smith, J.E., "Vortex analytical Model of a Circulation Controlled Vertical Axis Wind Turbine.” American Society of Mechanical Engineers, Energy Sustainability Conference, San Francisco, CA, 2009.

77. Wilkerson, J.B., Reader, K.R., Linck, D.W., "The Application of Circulation Control Aerodynamics to a Helicopter Rotor Model.” American Helicopter Society, 29th Annual Forum, Washington, D.C., 1973. 
78. Williams, R.M., "Some Aspects on Rotor Circulation Control.” California Avionics Laboratories, Inc., 3rd Symposium of Rotary-Wing and V/STOL Aircraft, Buffalo, NY, 1969.

79. Wood, N.J., "The Aerodynamics of Circulation Control Aerofoils.” Joint Institute for Aeronautics and Acoustics, Stanford University, Stanford, CA, 1981.

80. Zinger, Inc., “Zinger Propeller,” http://www.zingerpropeller.com/ Accessed: August 25, 2010. 


\section{Acknowledgements}

After a full decade of higher education, I would like to thank my family and friends for their reassurance and support, without which I could not have accomplished any of my goals. Special thanks to my wonderful wife, for being a sounding board to bounce ideas off of, a structural truss to lean on when things seemed to get out of hand, and for always being there to talk me down off an imaginary ledge when things seemed too difficult to deal with.

Highest regard and appreciation goes to my committee members, Dr. James E. Smith, Dr. MaryAnn Clarke, Dr. Kenneth H. Means, Dr. Andrew C. Nix, and Dr. William S. Wayne for their insight and technical help throughout the degree process which proved to be very beneficial. Dr. Victor H. Mucino and Dr. Jacky C. Prucz, who's departmental and graduate level guidance was instrumental in achieving all of the degrees which I pursued.

The current and past members of the Center for Industrial Research Applications (CIRA), and other graduate students who I have had the pleasure of working with, and who have had a lasting impact on my life professionally and personally, especially to Mr. Andrew D. Lowery and Mr. Michael S. Spencer for their electronics background, without which my experimental investigation would never have materialized, to Mr. Chad C. Panther for his aerodynamic expertise, to Mr. Kenneth Williams for his mechanical intuition, computer aided design skills, and hands-on background. Sincerest gratitude to a fellow Statler College of Engineering and Mineral Resources doctoral candidate, Mr. Christopher D. Griffin, for his assistance and background in computational fluid dynamics.

Special thanks to Dr. Gary L. Winn of the Industrial Engineering Department for providing employment opportunities while earning my degree. Thanks also to Dr. Gary J. Morris and Mr. Bruce Sparks from the West Virginia University Technology Transfer office who offered expertise and assistance in writing and submitting patent information and paperwork. 
I also appreciate the contributions that were made from outside sources, instilling the belief that the Statler College of Engineering at West Virginia University functions as a family, out to assist in any way possible. Among these people were Mr. Daniel K. Carder, Mr. Zachary J. Luzader, and Mr. Byron L. Rapp from the Center for Alternative Fuels, Engines, and Emissions who allowed some of their data acquisition elements to go missing for a few months. Mr. Charles D. Coleman and Mr. Clifford O. Judy of the Mechanical and Aerospace Engineering Department who's help in manufacturing and acquiring components was essential. Dr. Frances L. Vanscoy, Mr. Adam S. Dorsey, and Mr. Don E. McLaughlin from the Lane Department of Computer Science and Electrical Engineering, Dr. Charles B. Yuill of the Agricultural Sciences Department, and Ms. Laura Pitrolo of the Design and Merchandising Department who all consented to allow me to us their three-dimensional scanners early in my work in order to produce working computer models. Also assisting in some of the electronic work required during experimentation was Dr. Roy S. Nutter, of the Lane Department of Computer Science and Electrical Engineering.

Finally, to the private companies which donated time and resources to the entire unfunded project from the very beginning. Special thanks to Mr. Patrick Wildfire at Wilson Works Inc., Morgantown, WV, for acquisition of materials and industrial quality machining work, Mr. Chad Staller at Konica Minolta, Ramsey, NJ, for working to provide a threedimensional computer model of the propeller, Mr. Rich Sykes of Ametek Company, Arnold, MD, Mr. Aaron Blackmore and Mr. Darren Perry of Stratasys, Jeannette, PA all of whom helped render and print the final version of the model propeller that was tested in my experiment.

Also to Mr. Roger Rucker at United Technologies Corporation and Mr. Cale H. Zeune at Wright-Patterson Air Force Base in Dayton, OH, for affording me the opportunity to work as a research engineer for a summer and use the high-tech facilities at $A F R L$ to complete the final 
portions of my research. Tunnel research help at WPAFB was provided by Mr. Michael James, Mr. Kevin King, Mr. Jon Geiger, Dr. Michael V. Ol, and $2^{\text {nd }}$ Lt. Mitchell Haverkamp.

The final work could not have been completed without these contributions. For that reason I am grateful to all of these people for their sacrifice of their time, volunteering their expertise, and the addition of their countless resources to make this project a success. 


\section{Appendix A - Cincinnati SQAF - 270 Specifications}

This appendix shows the necessary paperwork and dimensions of the centripetal fan used in the experimental analysis. The fan is documented as a Cincinnati $S Q A F-270$ Airfoil Blower and was originally used as a driving mechanism on another experiment. It was necessary to us the inlet of the fan as the driving mechanism of this study and therefore was also required to do a characterization of the flow possibilities that were used to test the $C C$ propeller.

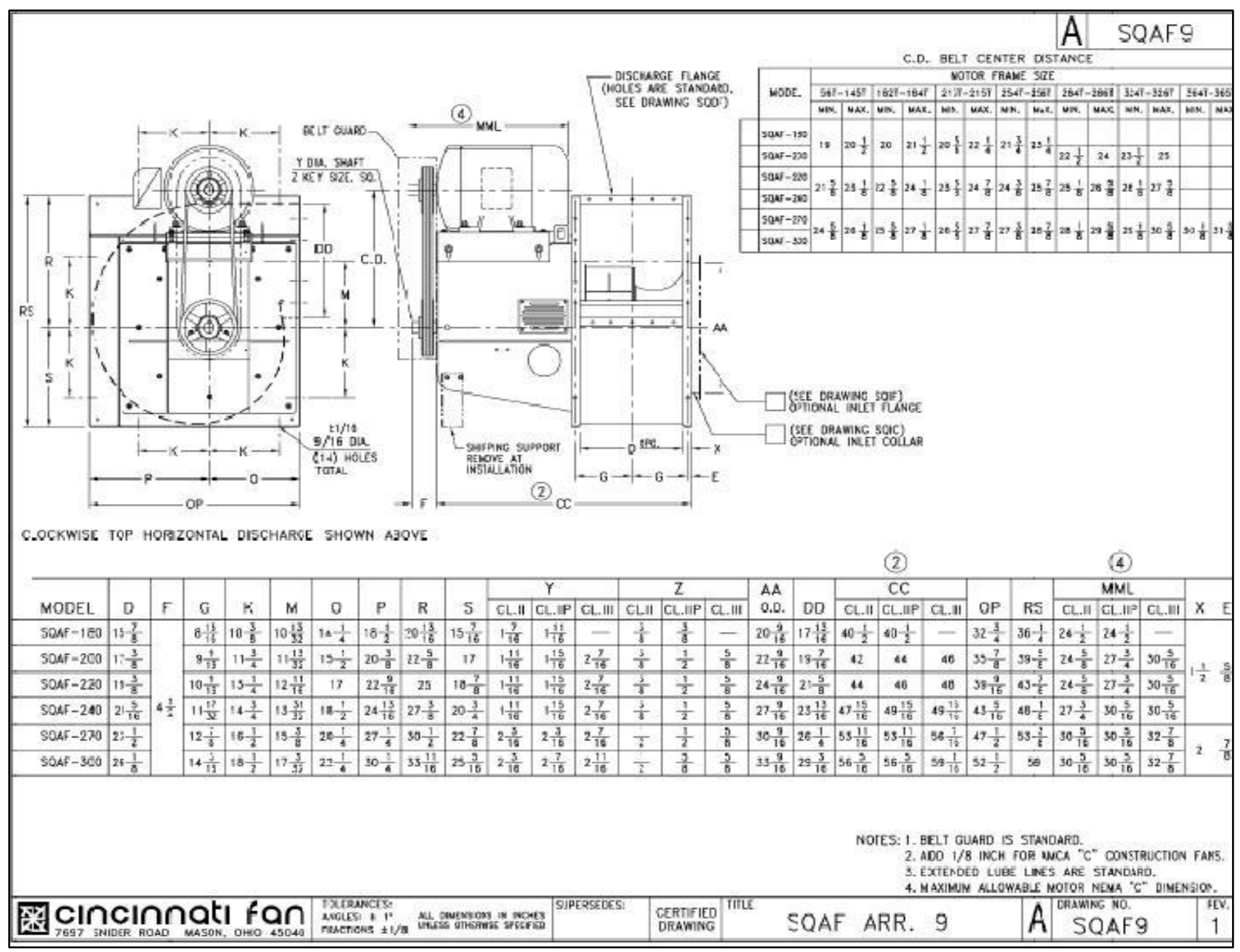

Figure 100. Cincinnati Fan Dimensions 


\section{Appendix B - Rapid Prototyping Machine Specifications}

This appendix identifies the necessary paperwork and specifications of the rapid prototyping machine used to make the experimental propeller. Marketed by Fortus, the 900mc lines of printers are accurate to within $0.128 \mathrm{~mm}$ (0.005 inches) and have the option to use any of seven different printing materials (Hansen, 2009). Each of these materials is spool-fed acrylonitrile-butadiene-styrene $(A B S)$ plastics which are super-heated then cured via ultraviolet light. The differences in usable materials are in tensile and impact strengths when the plastic has cured. For this application and experiment it was chosen to use ULTEM 9085 type material in order to try to endure the rotational forces the propeller would see when in motion. This material was tested to have the best overall tensile strength at 71.6 Mpa (10,390 psi) with the highest corresponding flexural strength of $115 \mathrm{Mpa}(15,100 \mathrm{psi})$. The specification sheets for these materials are shown below in Figure 101. 


\section{BASE SYSTEM CONFIGURATION}

Build Envelope (XYZ)

$36 \times 24 \times 36$ inch $(914.4 \times 609.6 \times 914.4 \mathrm{~mm})$

Platen supports two (Z) build zones for either a small or large build sheet

Material Delivery

Two (2) Build material canisters $92 \mathrm{in}^{3}(1508 \mathrm{cc}$ )

Two (2) Support material canisters $92 \mathrm{in}^{3}$ (1508 cc)

Auto changeover between canisters

\section{MATERIAL OPTIONS}

Layer Thickness:

a

ABS- $P C$ ABS I PCISOI

$P C$

ULTEM $^{\star}$
$9085^{\prime}$

0.013 inch $(0.330 \mathrm{~mm})$

0.010 inch $(0.254 \mathrm{~mm})$

0.007 inch $(0.178 \mathrm{~mm})$

Support Structure:

M3Oi

$x$

$\mathrm{X}$

\begin{tabular}{c|ccc}
$x$ & $x$ & $x$ & $x$ \\
$x$ & $x$ & $x$ & $x$
\end{tabular}

$x$
$x$

$x+x$

$x \quad x \quad x \quad x$

Soluble Soluble Soluble BASS BASS BASS BASS

Available Colors:

Soluble

Elwory
WWite
Black
Red
Blue
Dark

'Divory

Black

[.] Trans-

lucont

White

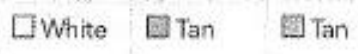

Grey

Figure 101. Specifications of Materials used in the FORTUS 900mc Rapid Prototyping Machine 


\section{Appendix C - Three-Dimensional Scanning Camera}

This appendix documents the specifications of the three-dimensional scanning camera used to generate a computer model of the un-augmented propeller. This camera, marketed by Konica Minolta, is called the Range 7 and uses non-contact digitizing to create an accurate computational representation of any real-life part or component without the hassle of measuring, checking and drawing the data first. The fully developed camera has an accuracy level of 40.0 $\mu \mathrm{m}$ (0.0016 inches) and takes 1.31 megapixels of data on each scan. Using targeted stickers, the scan dimensions of the parts can range small electronic components to cars and buildings. With the appropriate use of the scanning function and a target to line each scan up to there is no limit to the size of the object that can be scanned. The limiting factor is the user's computer memory to store the scanned files as the model is built. Listed specifications are given below, in Figure 102. 


\begin{tabular}{|c|c|c|c|c|c|}
\hline \multirow{2}{*}{\multicolumn{2}{|c|}{$\begin{array}{l}\text { Measuring method } \\
\text { Light source }\end{array}$}} & \multicolumn{4}{|c|}{ Triangulation by light sectioning method } \\
\hline & & \multicolumn{4}{|c|}{ Semiconductor laser, Wavelength: $660 \mathrm{~nm}$} \\
\hline \multicolumn{2}{|c|}{ Laser class } & \multicolumn{4}{|c|}{ Class 2 (IEC 60825-1 Edition 2) } \\
\hline \multicolumn{2}{|c|}{ Number of pixels taken } & \multicolumn{4}{|c|}{1.31 megapixels $(1280 \times 1024)$} \\
\hline \multicolumn{2}{|c|}{ Measurement distance } & \multicolumn{4}{|c|}{450 to $800 \mathrm{~mm}$} \\
\hline \multicolumn{2}{|c|}{$\begin{array}{l}\text { Receiving lens } \\
\text { (interchangeable) }\end{array}$} & \multicolumn{4}{|l|}{ TELE, WIDE } \\
\hline \multirow{3}{*}{$\begin{array}{l}\text { Measure- } \\
\text { ment } \\
\text { range } \\
\text { (mm) }\end{array}$} & Direction & $\begin{array}{l}\text { TELE } \\
450 \mathrm{~mm}\end{array}$ & $\begin{array}{l}\text { TELE } \\
800 \mathrm{~mm}\end{array}$ & $\begin{array}{l}\text { WIDE } \\
450 \mathrm{~mm}\end{array}$ & $\begin{array}{l}\text { WIDE } \\
800 \mathrm{~mm}\end{array}$ \\
\hline & $\mathrm{X} \times \mathrm{Y}$ & $79 \times 99$ & $141 \times 176$ & $150 \times 188$ & $267 \times 334$ \\
\hline & $\mathbf{z}$ & 54 & 97 & 109 & 194 \\
\hline \multicolumn{2}{|c|}{$\begin{array}{l}\mathrm{XY} \text { direction } \\
\text { measurement interval }\end{array}$} & 0.08 & 0.14 & 0.16 & 0.28 \\
\hline \multicolumn{2}{|c|}{$\begin{array}{l}\text { Accuracy (Distance } \\
\text { between balls) }{ }^{* 1}\end{array}$} & \multicolumn{4}{|l|}{ $\pm 40 \mu \mathrm{m}$} \\
\hline \multicolumn{2}{|c|}{ Precision $(\mathbf{Z}, \sigma)^{* 2}$} & \multicolumn{4}{|l|}{$4 \mu \mathrm{m}$} \\
\hline \multicolumn{2}{|c|}{ Auto-focus } & \multicolumn{4}{|l|}{ Provided } \\
\hline \multicolumn{2}{|c|}{ Auto-exposure } & \multicolumn{4}{|l|}{ Provided } \\
\hline \multicolumn{2}{|c|}{ Scan time } & \multicolumn{4}{|c|}{ Approx. 2 sec. or more (1 scan) } \\
\hline \multicolumn{2}{|c|}{ Preview function } & \multicolumn{4}{|c|}{ Approx. 0.4 sec./scan } \\
\hline \multicolumn{2}{|c|}{$\begin{array}{l}\text { Scanning ambient } \\
\text { lighting condition }\end{array}$} & \multicolumn{4}{|c|}{$500 \mathrm{~lx}$ or less } \\
\hline \multicolumn{2}{|c|}{ Output interface } & \multicolumn{4}{|c|}{ USB 2.0 High Speed } \\
\hline \multicolumn{2}{|l|}{ Power } & \multicolumn{4}{|c|}{$\begin{array}{l}\text { Included AC Adapter } \\
\text { Input voltage: } 100 \text { to } 240 \mathrm{VAC}(50 / 60 \mathrm{~Hz}) \text {, Rating: } 1.4 \mathrm{~A} \text { (at } 100 \mathrm{VAC} \text { input) }\end{array}$} \\
\hline \multicolumn{2}{|c|}{ Dimensions } & \multicolumn{4}{|c|}{$295(\mathrm{~W}) \times 190(\mathrm{H}) \times 200(\mathrm{D}) \mathrm{mm}$ (Excluding grips and lens) } \\
\hline \multicolumn{2}{|l|}{ Weight } & \multicolumn{4}{|c|}{ Approx. $6.7 \mathrm{~kg}$} \\
\hline $\begin{array}{l}\text { Operat } \\
\text { ture/hur }\end{array}$ & $\begin{array}{l}\text { tempera- } \\
\text { ty range }\end{array}$ & 10 to $40^{\circ} \mathrm{C}$; & re humidit & r less (no c & nsation) \\
\hline $\begin{array}{l}\text { Storag } \\
\text { ture/hu }\end{array}$ & $\begin{array}{l}\text { empera- } \\
\text { ity range }\end{array}$ & $\begin{array}{l}-10 \text { to } 50^{\circ} \mathrm{C} \text {; } \\
\text { (at } 35^{\circ} \mathrm{C} \text {, no }\end{array}$ & $\begin{array}{l}\text { le humidit } \\
\text { nsation) }\end{array}$ & or less & \\
\hline
\end{tabular}

Figure 102. Specifications of the Konica Minolta Range 7 Laser Scanner 


\section{Appendix D - Rotational Study of Augmented Propeller}

This section illustrates the study of the propeller in a rotational fashion in order to predict when failure would occur in the blades. A simple rotational analysis at several rotational speeds was conducted in Pro-ENGINEER software and compared to the strength of the printed materials, ULTEM and polycarbonate. The propeller model was predicted to fail between 6000 and $7000 \mathrm{rpm}$, about $2000 \mathrm{rpm}$ less than the rated speed for the un-augmented propeller. This was due to the fact that there was no designed support material inside the augmented propeller. In all the cases below, it is shown that the maximum displacement occurs around the circulation control exit slot while the maximum stresses are seen to happen just above the mating point between the blades and the central hub. Some of the results of analysis runs conducted are shown in Figure 103, Figure 105, and Figure 107, specifically showing the stress concentrations acting on the blades at each rotational speed of (2000, 4000, and $9000 \mathrm{rpm})$. Similarly, Figure 104, Figure 106, and Figure 108 show the physical deformation predicted to occur on the propeller blades due to each of the analyzed rotational speed (also studied at 2000, 4000, and $9000 \mathrm{rpm}$ ). 


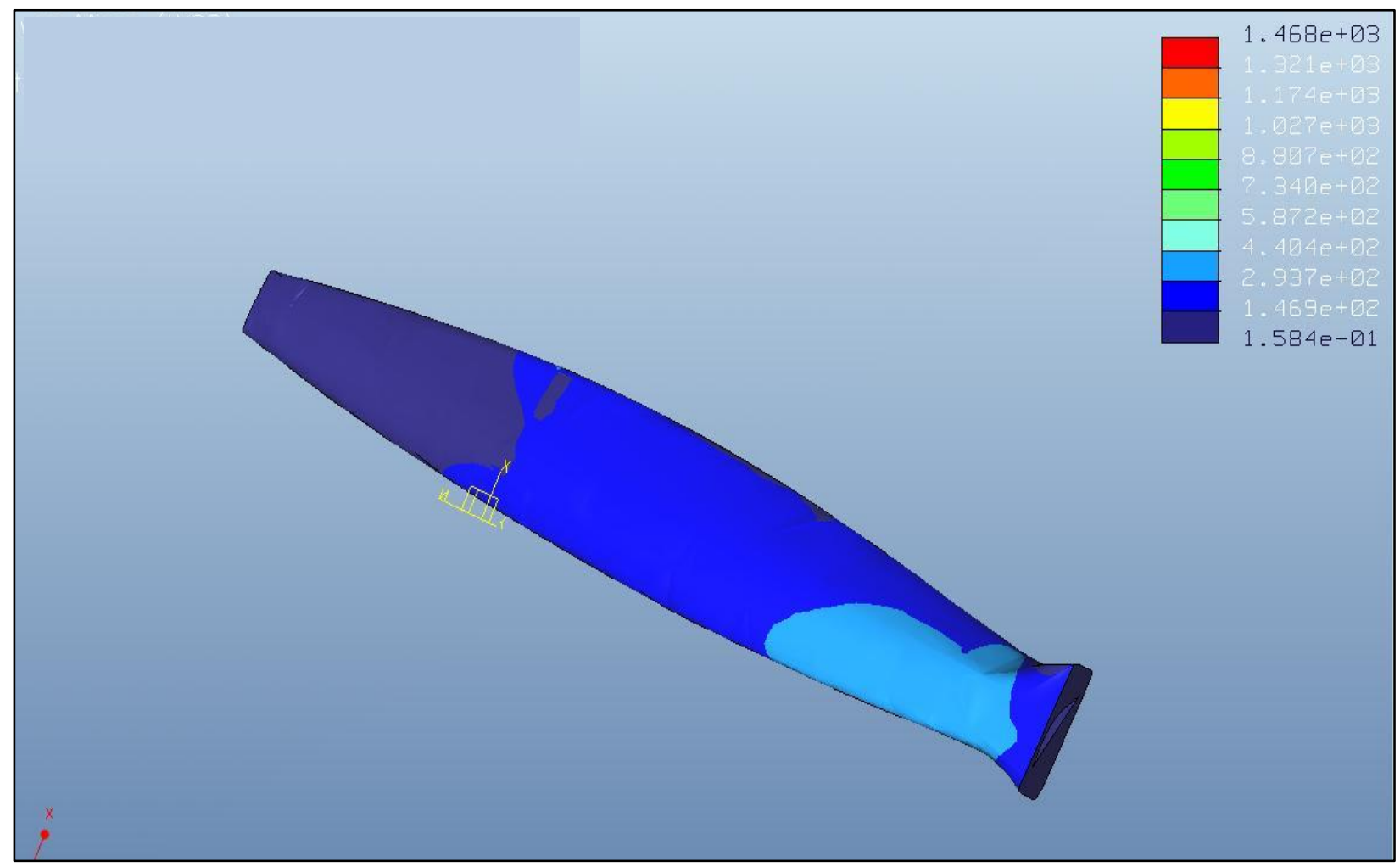

Figure 103. Stress Concentrations at 2000 rpm

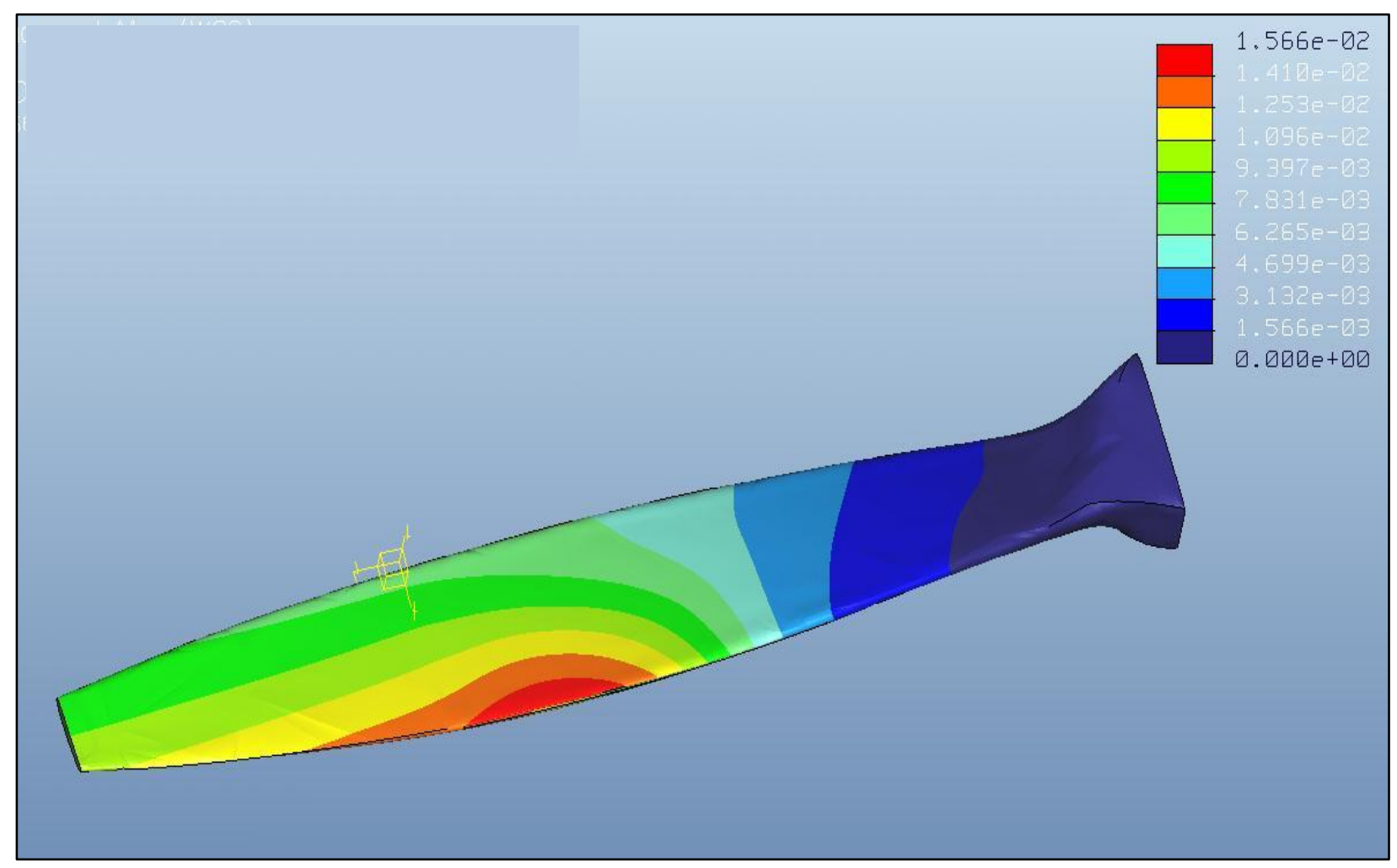

Figure 104. Displacement at 2000 rpm 


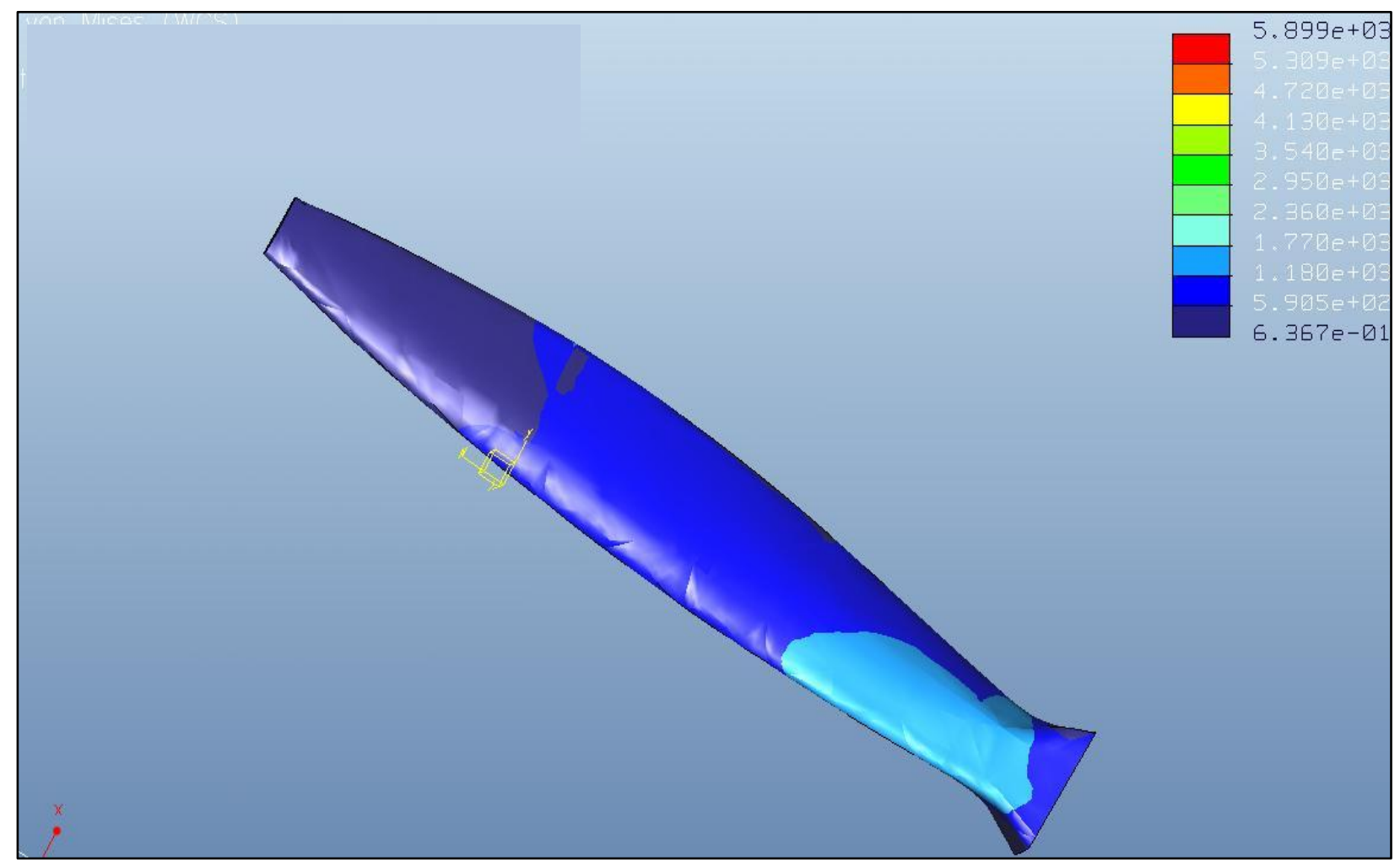

Figure 105. Stress Concentrations at 4000 rpm

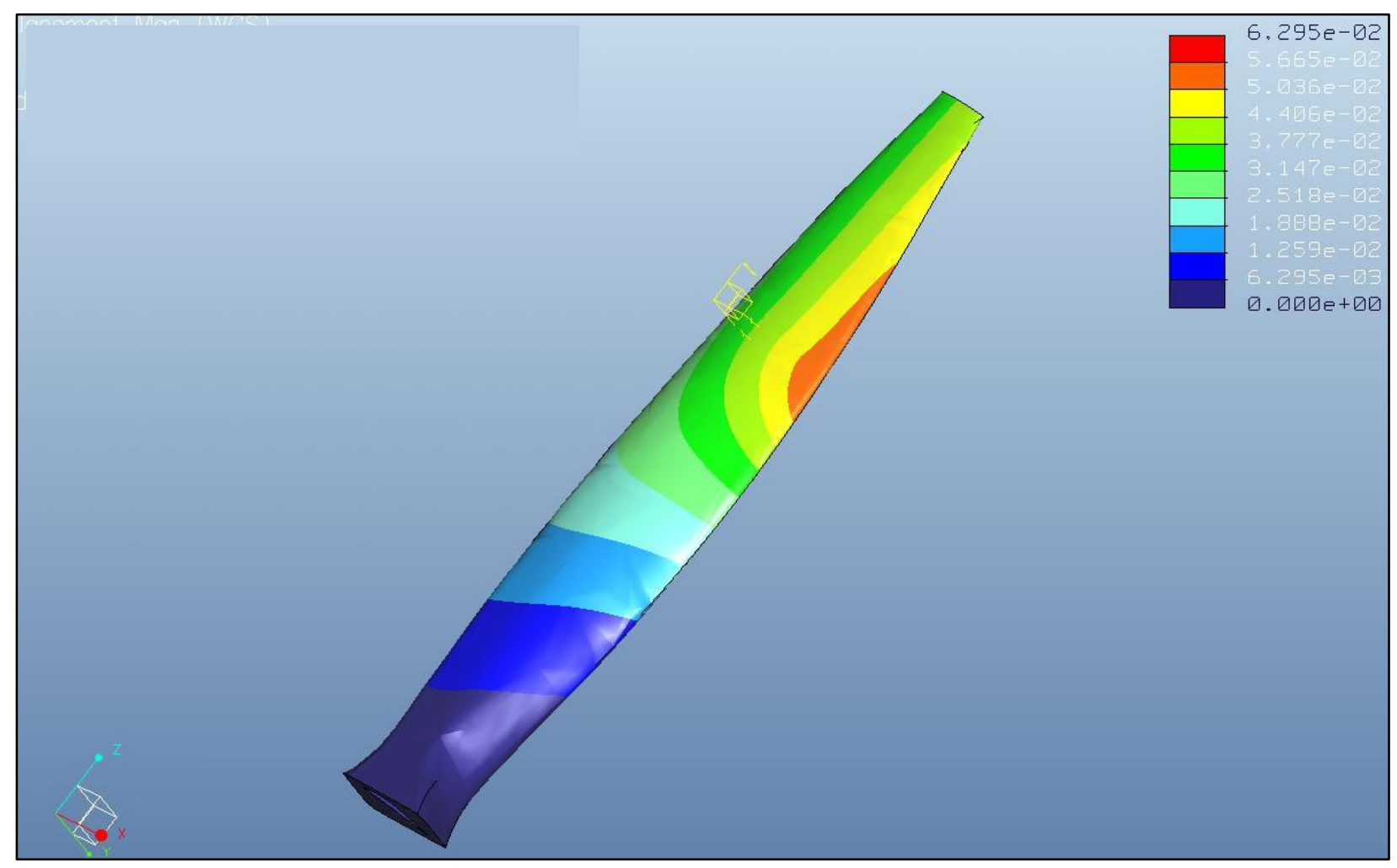

Figure 106. Displacement at 4000 rpm 


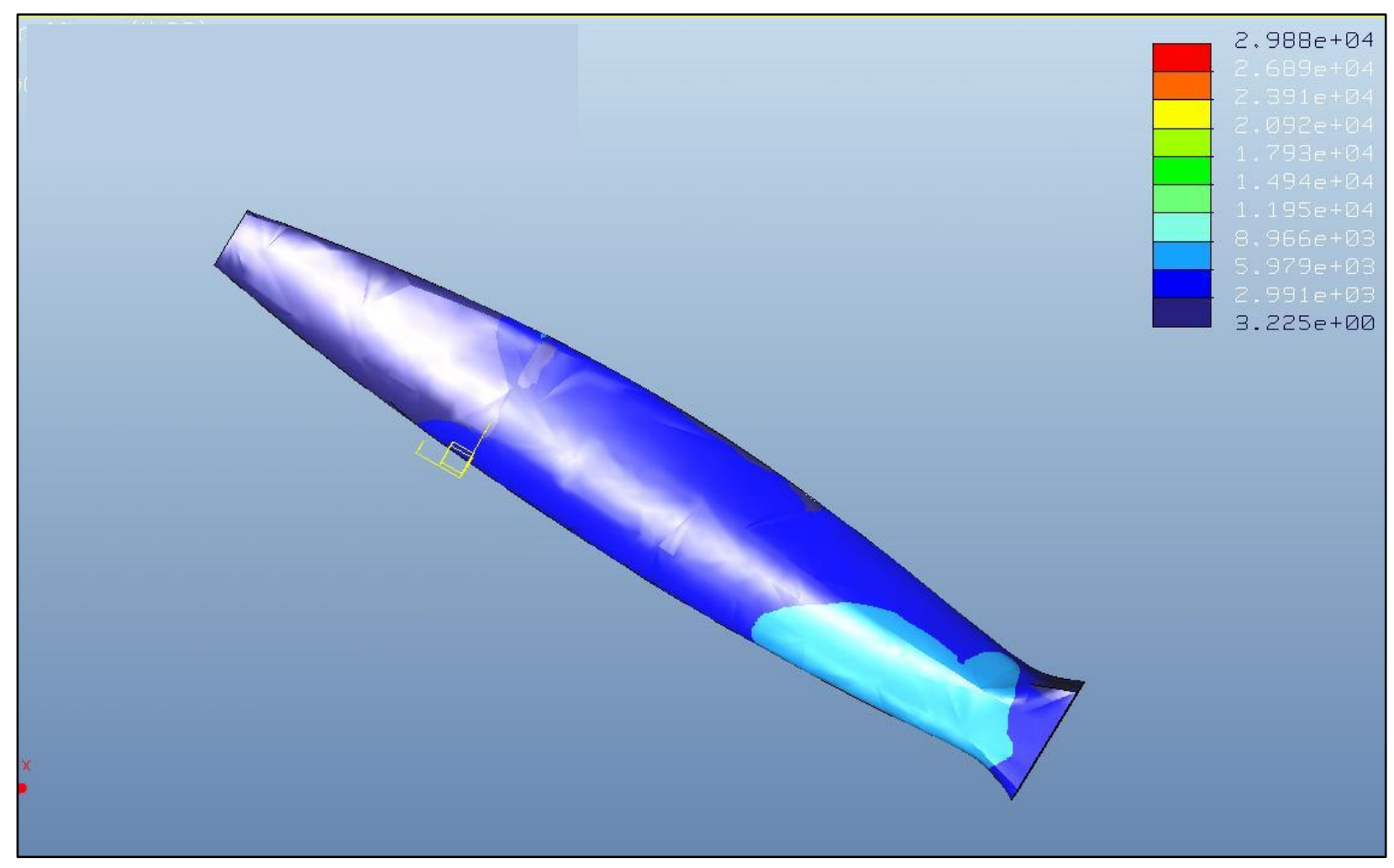

Figure 107. Stress Concentrations at 9000 rpm (Failure Predicted)

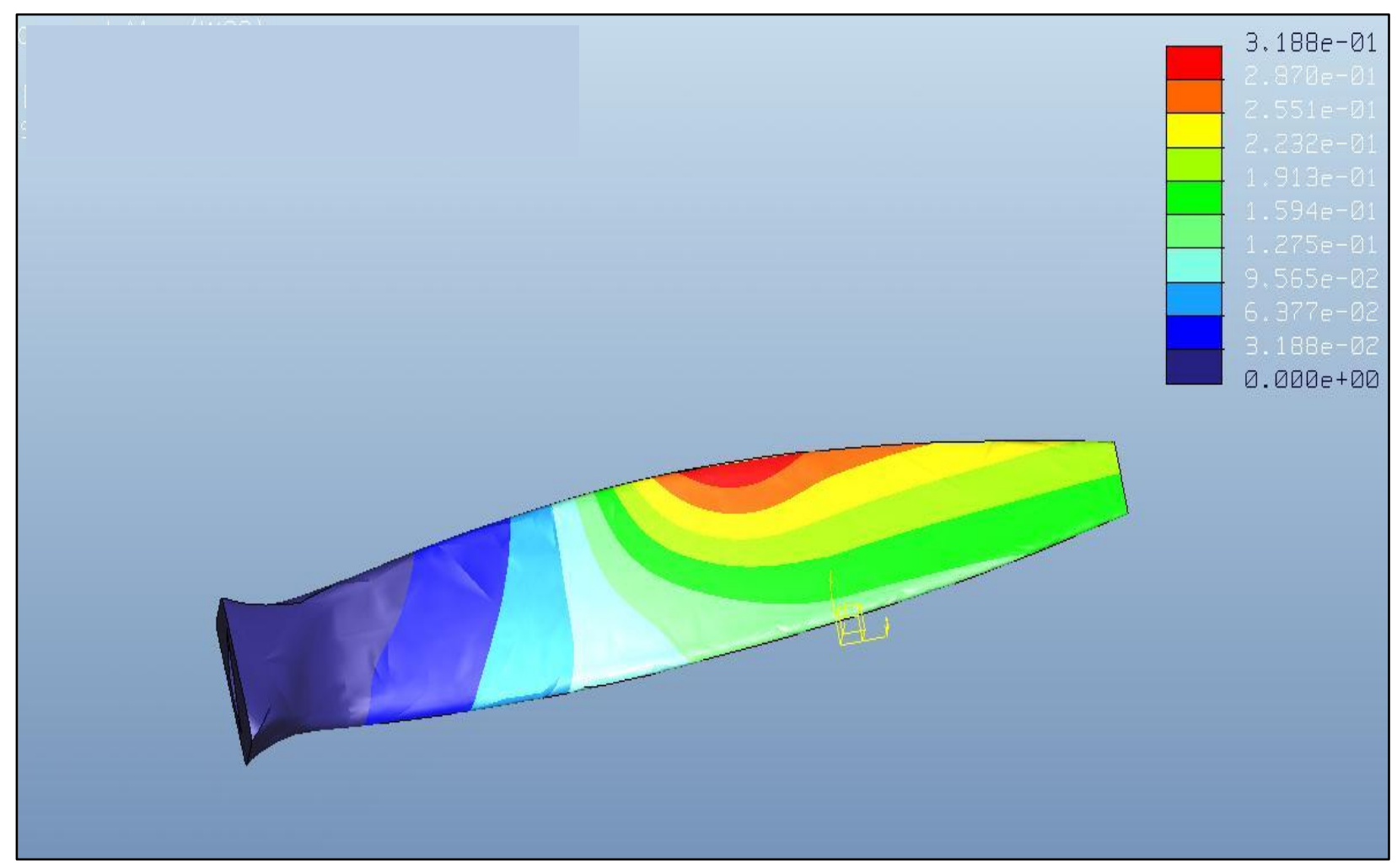

Figure 108. Displacements at $9000 \mathrm{rpm}$ (Failure Predicted) 


\section{Appendix E - Brushless Motor Specifications}

The specifications of the Rimfire brushless electric motor are given in the specification sheet listed below, in Figure 109. The values for input voltage and current were useful when trying to come with a power supply design for this experimental set-up, as were the physical dimensions when trying to design a test stand while the motor was being shipped.

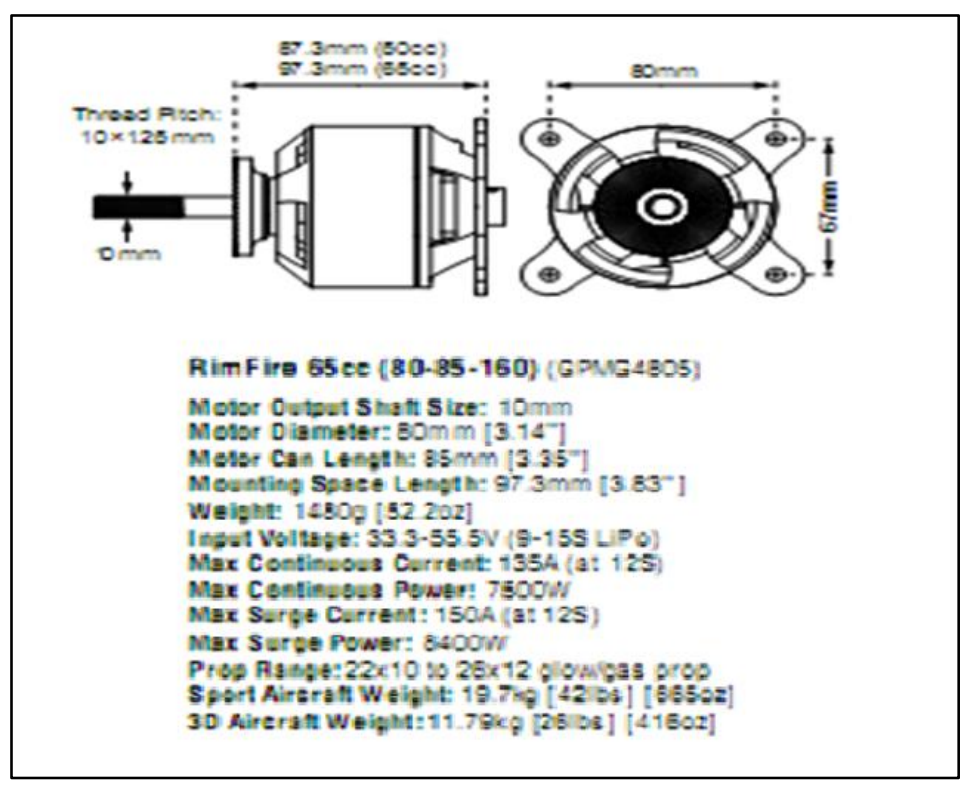

Figure 109. Brushless Motor Specifications 


\section{Appendix F - Electronic Speed Controller Specifications}

The electronic speed controller is a typical component used to program the electric motor when using them on a remote control aircraft. Consequently, in order to vary the input voltage to the motor in order to examine the rotational speeds of the propeller, this was another necessary component of the motor experimental set up which needed to be chosen and sized according to the motor selection. These units are meant to provide the battery voltage (normally a $D C$ current) a conversion to alternating current and voltage to feed into the three phase motor. The $E S C$ also allows for full control of the motor from an outside source, meaning that the ramp up time, brake ratio for stopping purposes, and overall speed ratings can all vary given input voltage values to the motor. Figure 110 shows a wiring diagram of the chosen ESC (Hobbywing Platinum Pro) used to mate with the Rimfire motor.

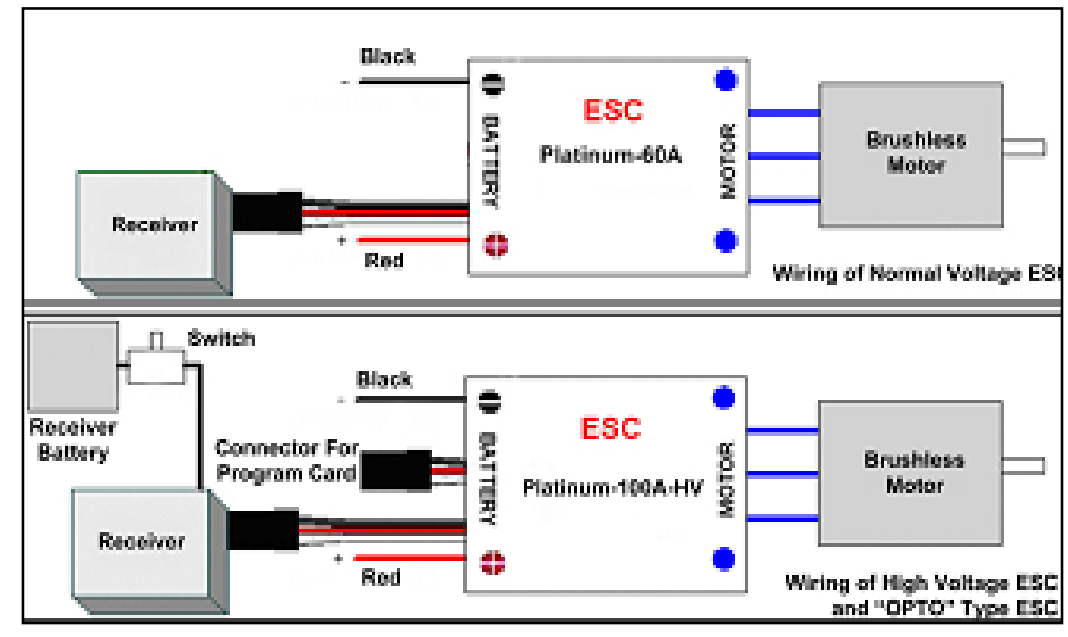

Figure 110. Hobbywing Platinum Pro ESC Wiring Diagram

Figure 111 shows a typical governor mode rotational speed analysis based on the rpm of the motor and the resultant throttle volume given by the ESC. In the "low mode" of governor control, the ESC will read input throttle volume and convert it to a rotational speed which is in a range from zero rpm to about half of the maximum rotational speed value. This allows for greater input control at lower end rotational speeds. Conversely, in "high mode" the ESC will 
take input voltage and allow the motor to cycle through any of the range of possible rotational speed values possible based on the throttle input volume.

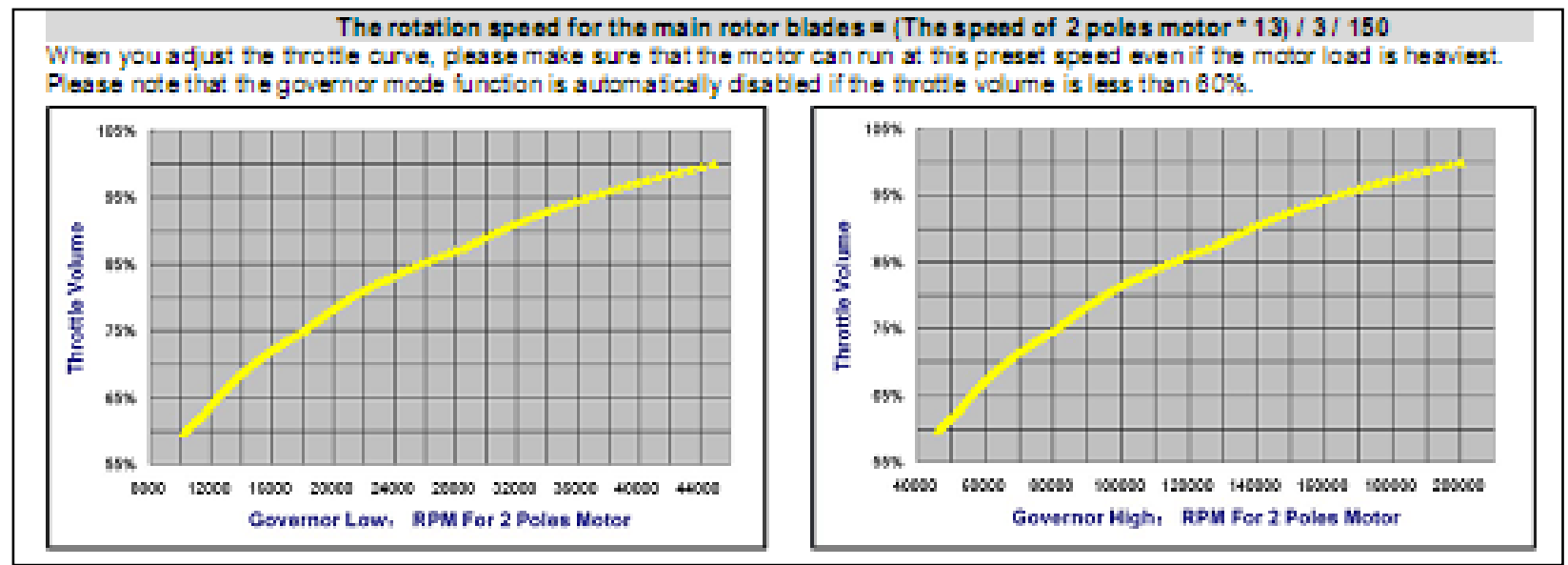

Figure 111. Governor Mode Throttle Volume

A programming box was use to communicate from the computer interface directly to the $E S C$ without the need to program the ESC with MATLAB or Labview software, and also allowed for easy transition of several input parameters without losing research time reprogramming a primitive program. It also allowed for more accurate programming in the rotational speed of the motor as the program box was specifically programmed to mate with the chosen ESC. Programmable inputs through this program box included: running mode, low-voltage cut off, rotational direction, battery cell type and number, start-up mode, throttle control, overheat protection, and brake forces. A wiring description is shown in

Figure 112. This program box was used to translate a series of beeping codes from the $E S C$ to a digital readout of what the settings were defined during the experimental operation. 


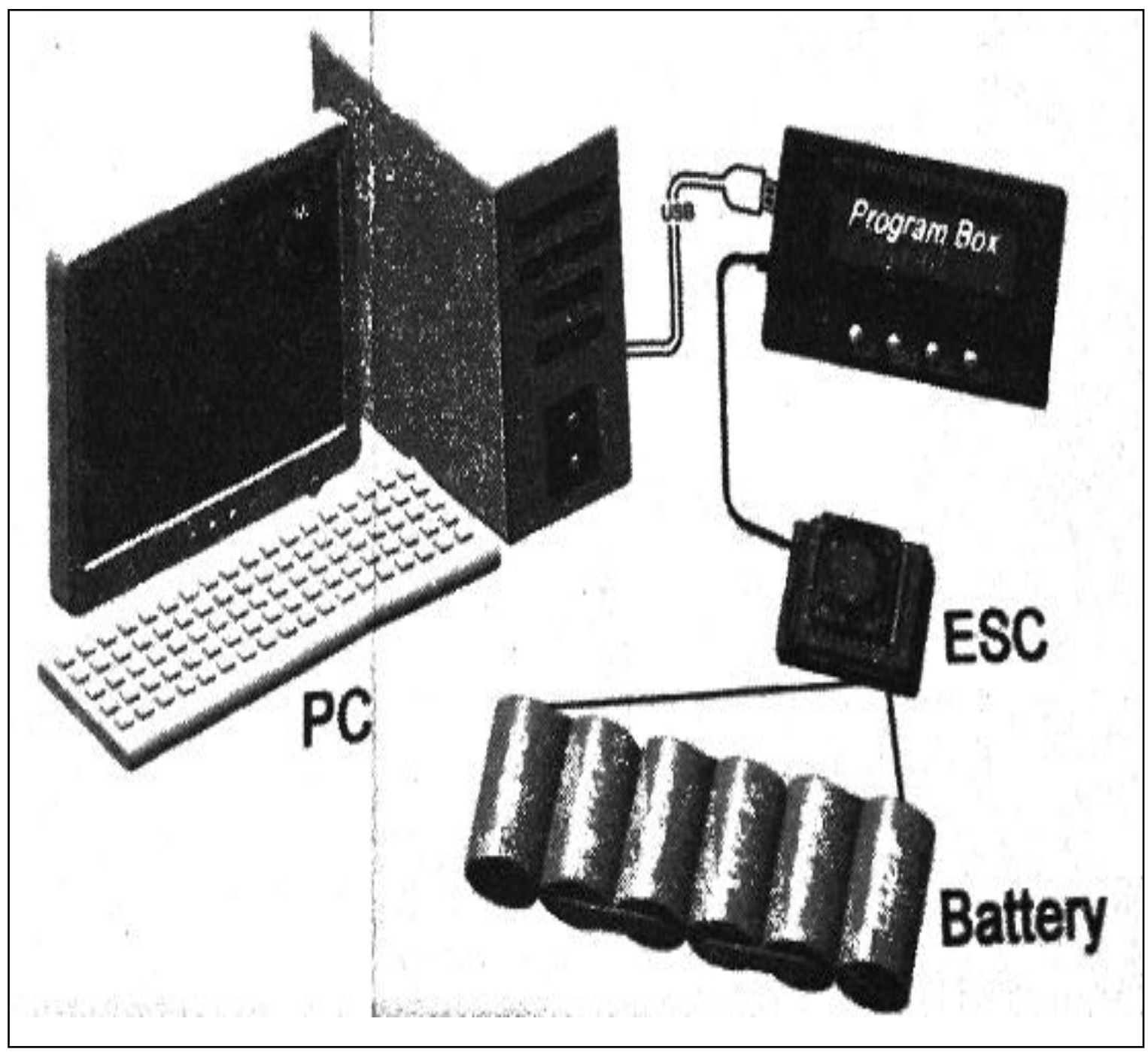

Figure 112. Computer to Program Box to ESC Wiring Diagram

A second $E S C$ was used to conduct experiments at the $W P A F B$ wind tunnel, primarily because the data acquisition system was already installed and the power supply was also already provided, without the need to use car batteries. In these experiments, a Castle Creations, Phoenix ICE 50 amp ESC was used and was also compatible with the Castle Link data logger which was used as a backup data acquisition system to judge the input and output parameters after each run without the need to reduce all the data immediately. The Castle Link monitored input parameters (current, voltage and power) to the motor as well as output rotational speeds 
and plotted these values in real-time charts against the time of the experiment. See results in Appendix L - Castle Link Speed Controller Data Logger. This ability became very powerful in between experimental trial as the data from the tunnel force balance and anemometers did not have to be analyzed right away. By downloading the data from the Castle Link via USB and relating the output parameters to the experimental designed information, time in the tunnel was used most effectively to obtain the greatest amount of data possible in a limited amount of available experimental time. The data logger (Castle Link) is shown in Figure 113, while the dimensions of the Phoenix ESC can be found in Figure 114, below.

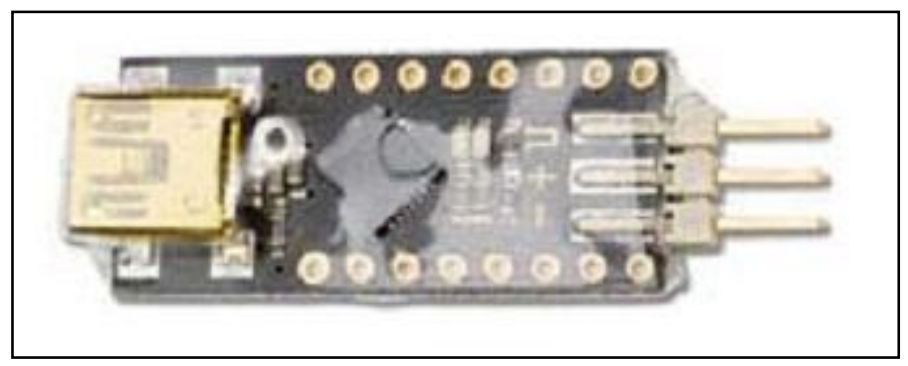

Figure 113. Castle Link USB Data Logger 


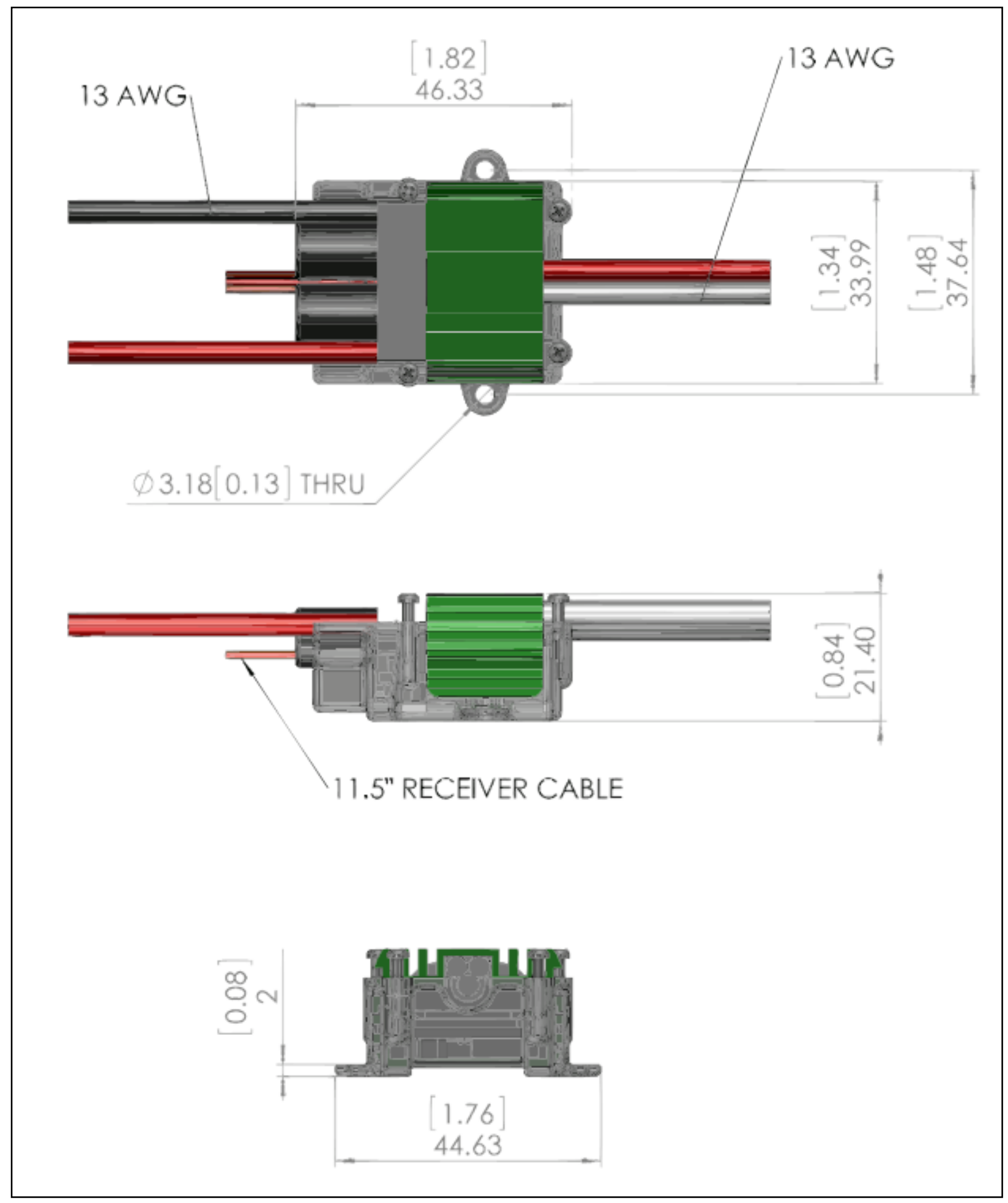

Figure 114. Castle Creations Phoneix ICE-50 Electronic Speed Controller 


\section{Appendix G - Clark-Y Simulated Airfoil Data}

Using a two-dimensional airfoil geometry analysis tool (DesignFoil), several estimations of airfoil section lift and drag coefficient data was estimated across a range of Reynolds Numbers $(90 \mathrm{k}-900 \mathrm{k})$ as well as angles-of-attack (0.0-20.0 degrees). This data was then used to estimate blade loading in a blade element analysis. Shown in this appendix are the results of the data runs across the angle-of-attack spectrum for the highest and lowest available Reynolds Number cases analyzed, 90,000 and 900,000, respectively.

\section{Angle-of-Attack Sweep at $R E=90,000$}

The following data set was analyzed as an angle-of-attack sweep done at a Reynolds Number of 90,000. Below are the figures which estimate the two-dimensional lift and drag profiles for each $A O A$ analyzed (0.0-20.0 degrees).

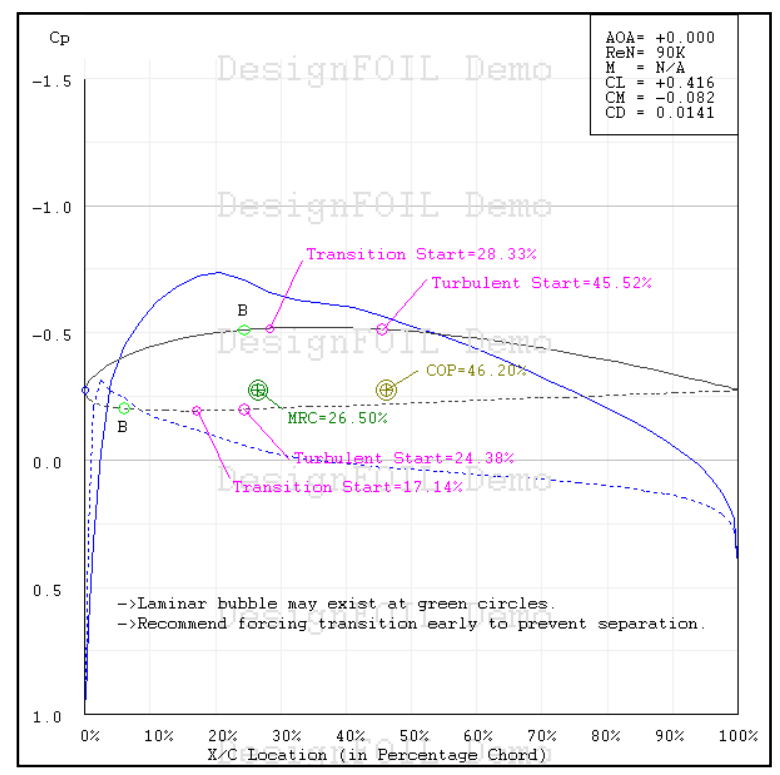

Figure 115. 2-D Airfoil Data at $0^{\circ} A O A$

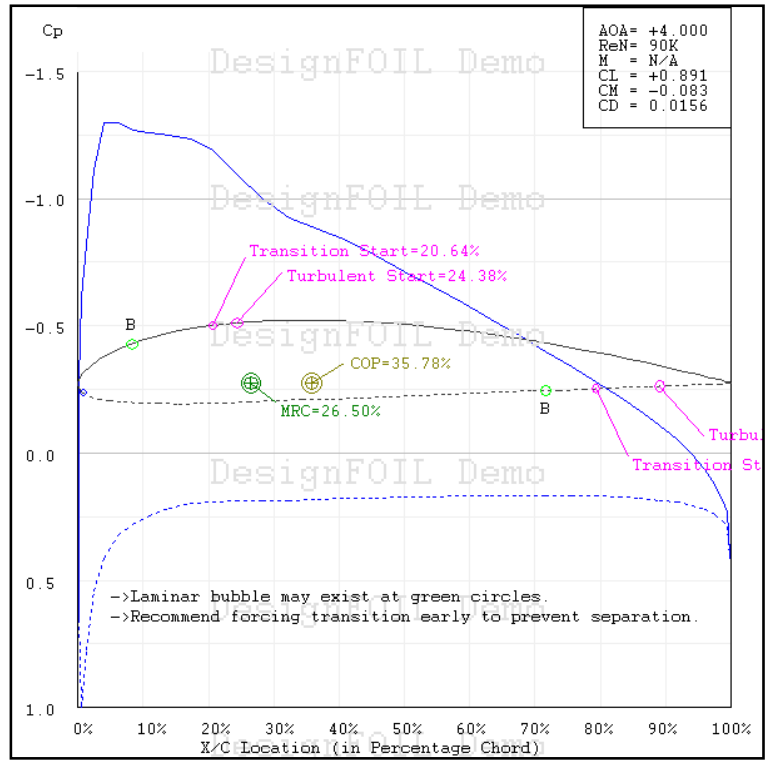

Figure 116. 2-D Airfoil Data at $2^{\circ} A O A$ 


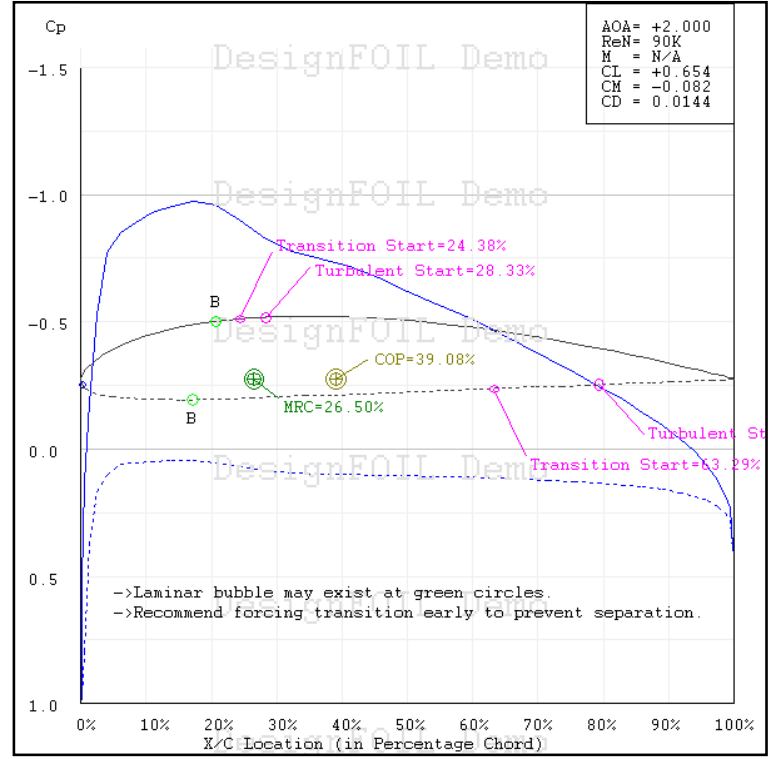

Figure 117. 2-D Airfoil Data at $4^{\circ} A O A$

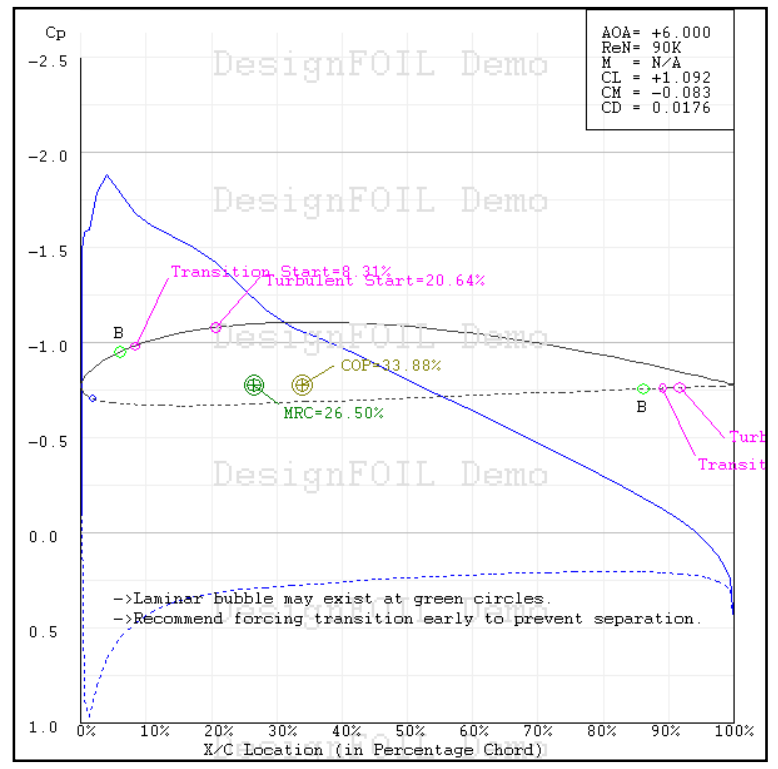

Figure 118. 2-D Airfoil Data at at $6^{\circ} A O A$

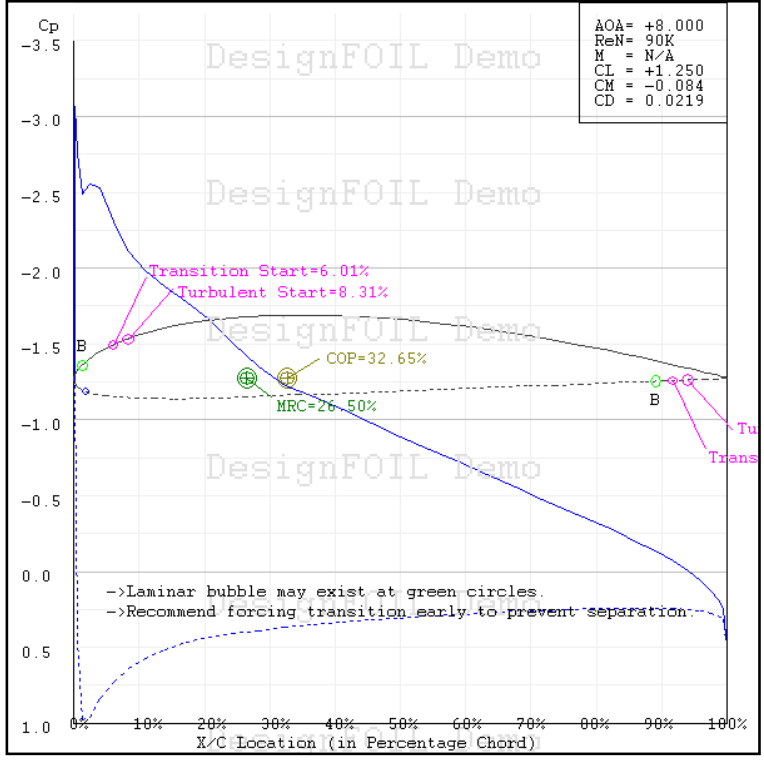

Figure 119. 2-D Airfoil Data at $8^{\circ} A O A$

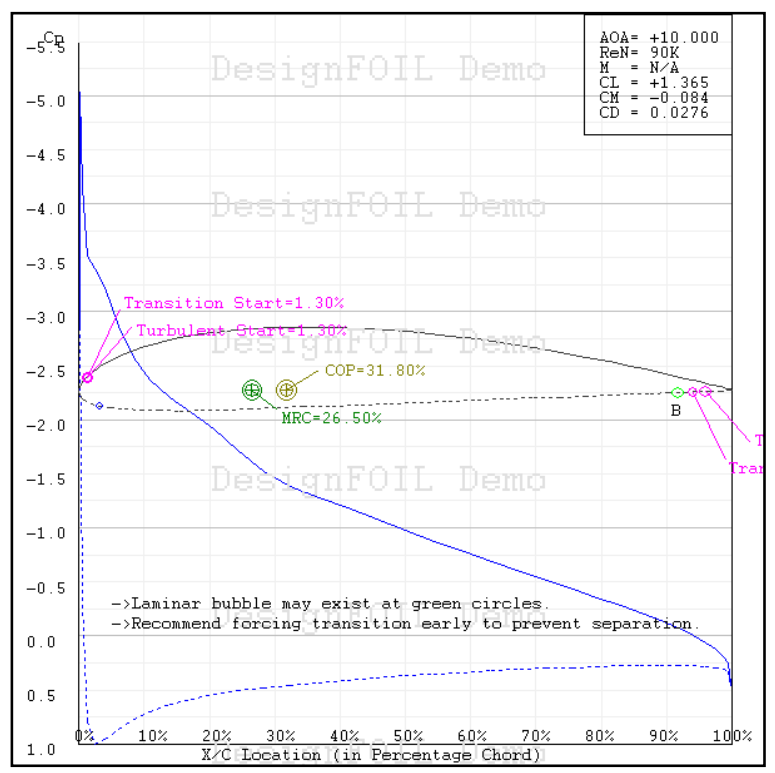

Figure 120. 2-D Airfoil Data at $10^{\circ} A O A$ 


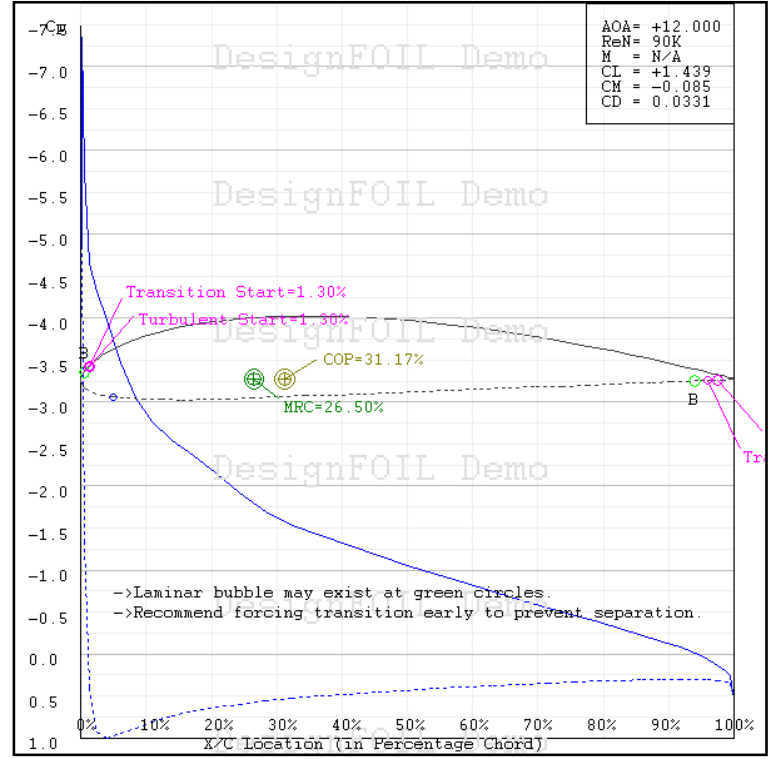

Figure 121. 2-D Airfoil Data at $12^{\circ} \mathrm{AOA}$

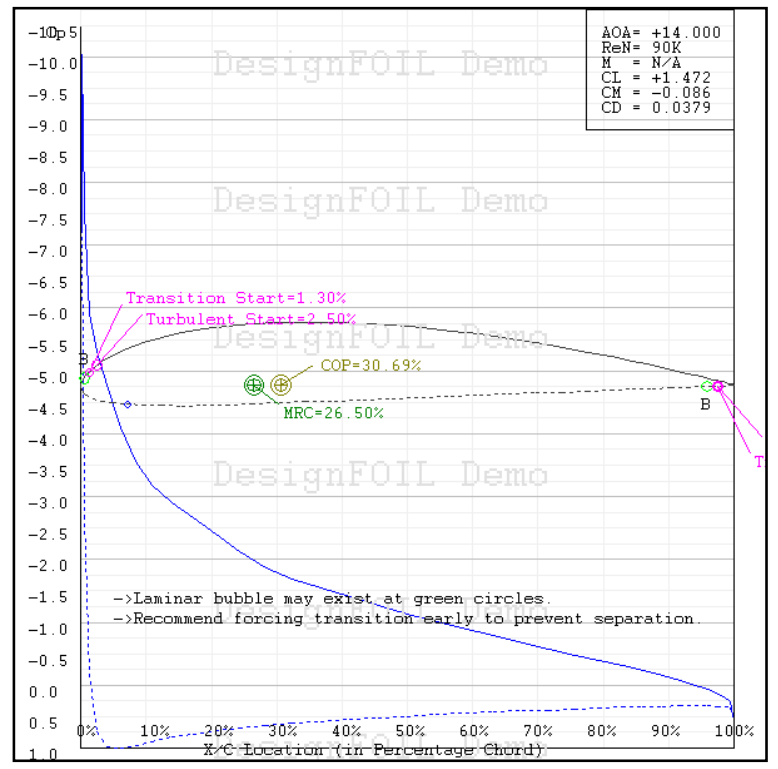

Figure 122. 2-D Airfoil Data at $14^{\circ} \mathrm{AOA}$

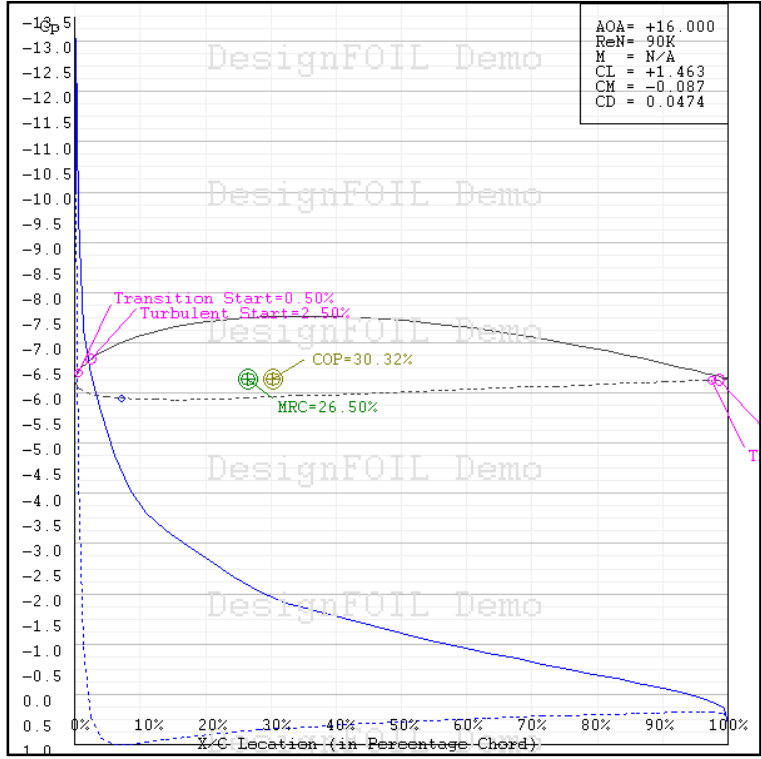

Figure 123. 2-D Airfoil Data at $16^{\circ} \mathrm{AOA}$

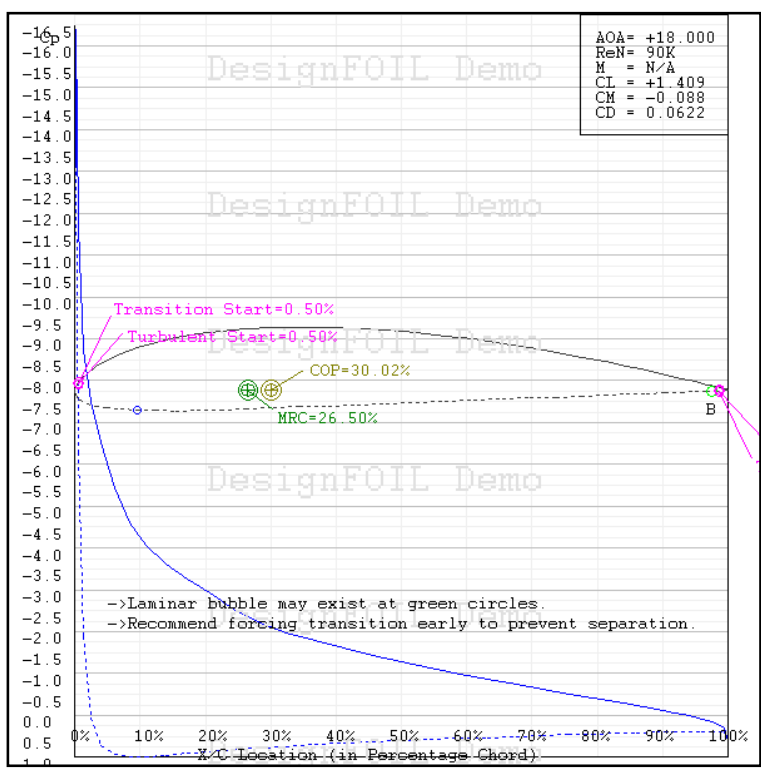

Figure 124. 2-D Airfoil Data at $18^{\circ} A O A$ 


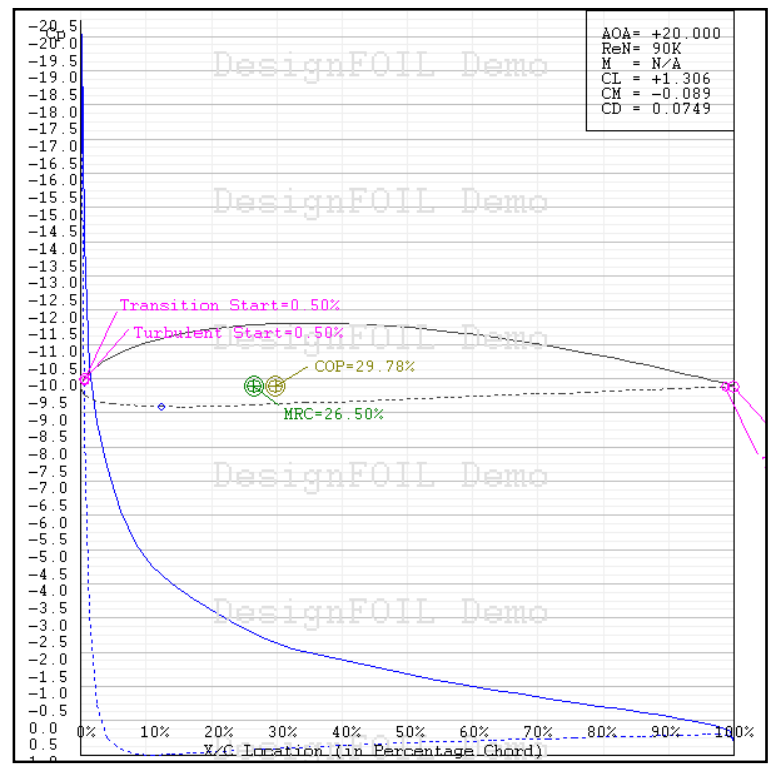

Figure 125. 2-DAirfoil Data at $20^{\circ} A O A$ 


\section{Angle-of-Attack Sweep at $R E=900,000$}

Similar to the previous data, another angle-of-attack sweep analysis was done at a Reynolds Number of 900,000. Below are the figures which estimate the two-dimensional lift and drag profiles for each $A O A$ analyzed (0.0-20.0 degrees).

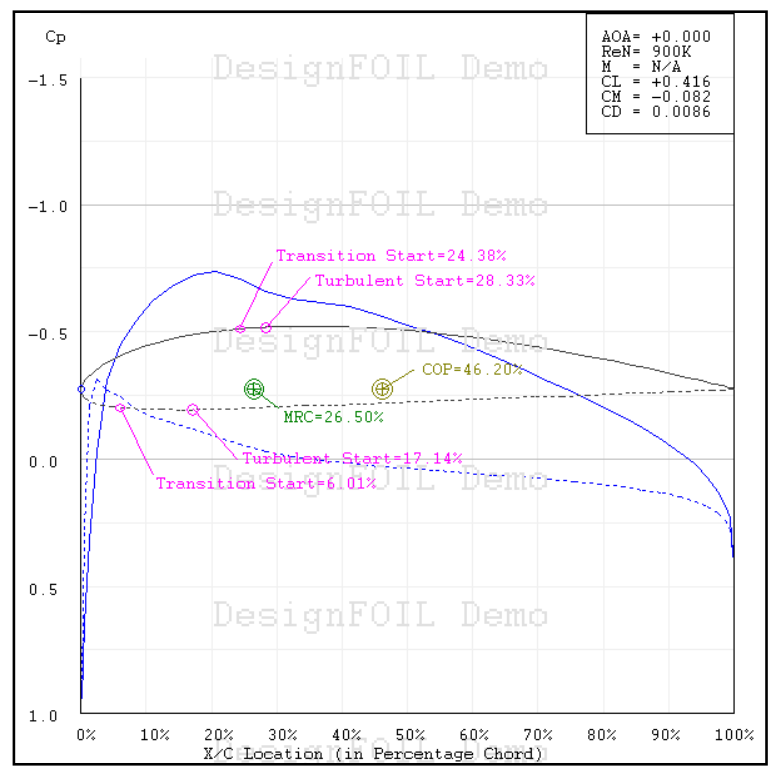

Figure 126. 2-D Airfoil Data at $0 A O A$

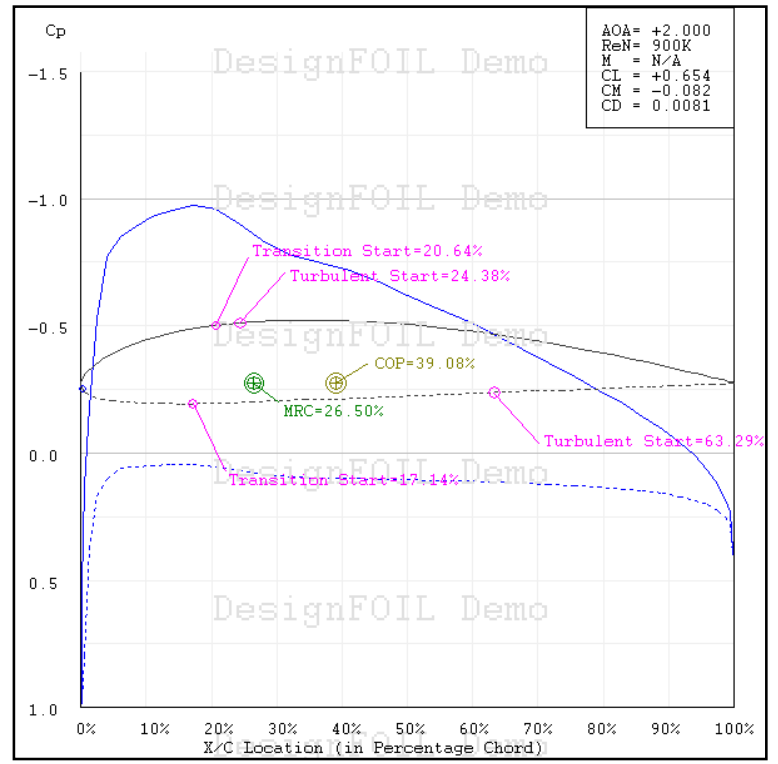

Figure 127. 2-D Airfoil Data at $2 A O A$

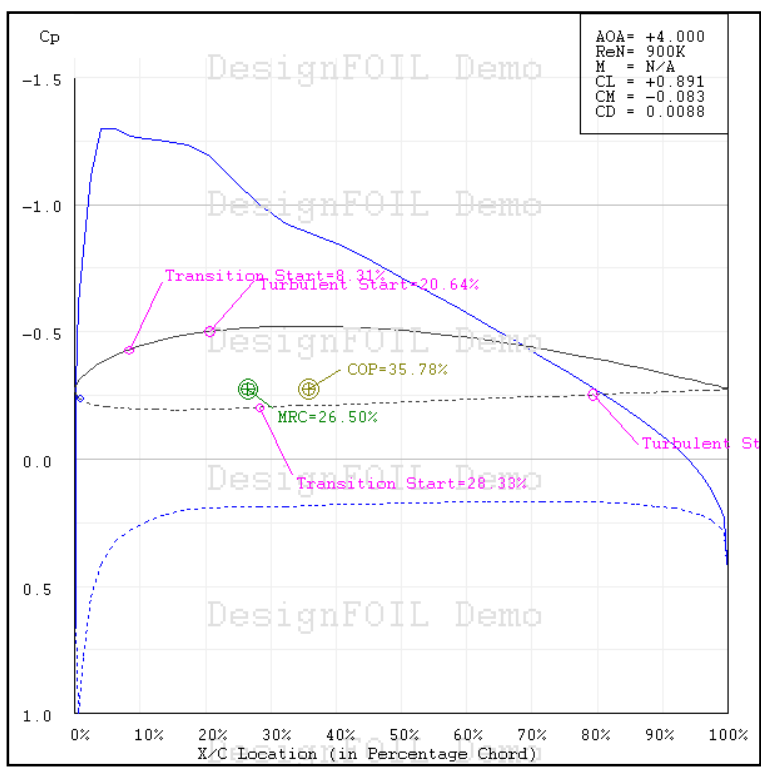

Figure 128. 2-D Airfoil Data at $4 A O A$

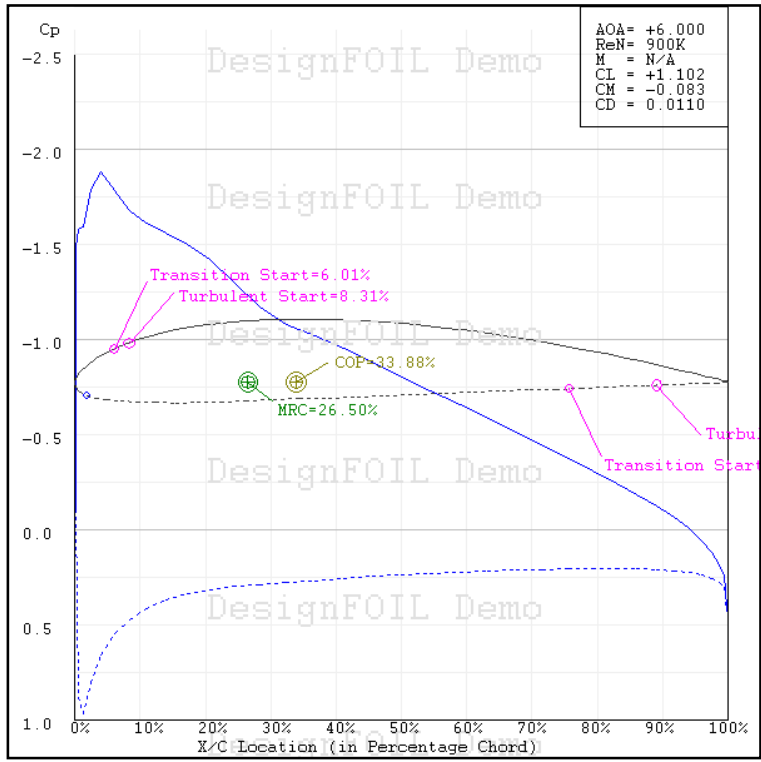

Figure 129. 2-D Airfoil Data at $6 \mathrm{AOA}$ 


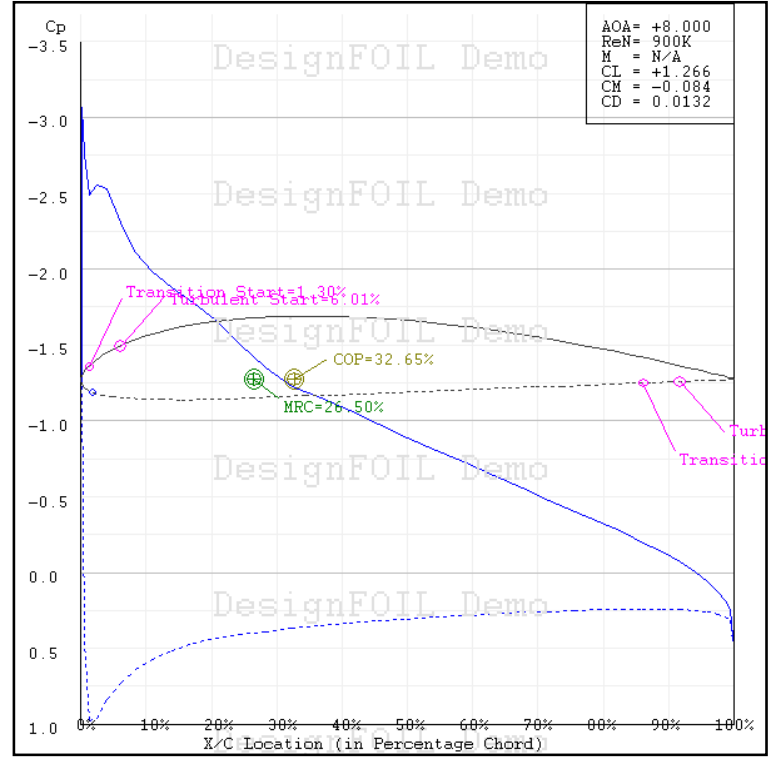

Figure 130. 2-D Airfoil Data at $8 \mathrm{AOA}$

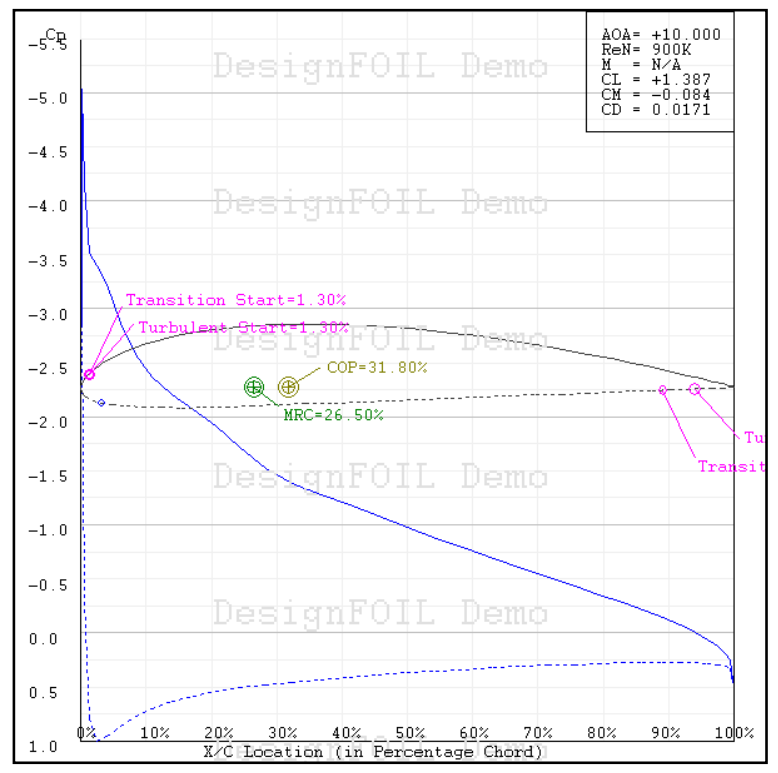

Figure 131. 2-D Airfoil Data at 10 AOA

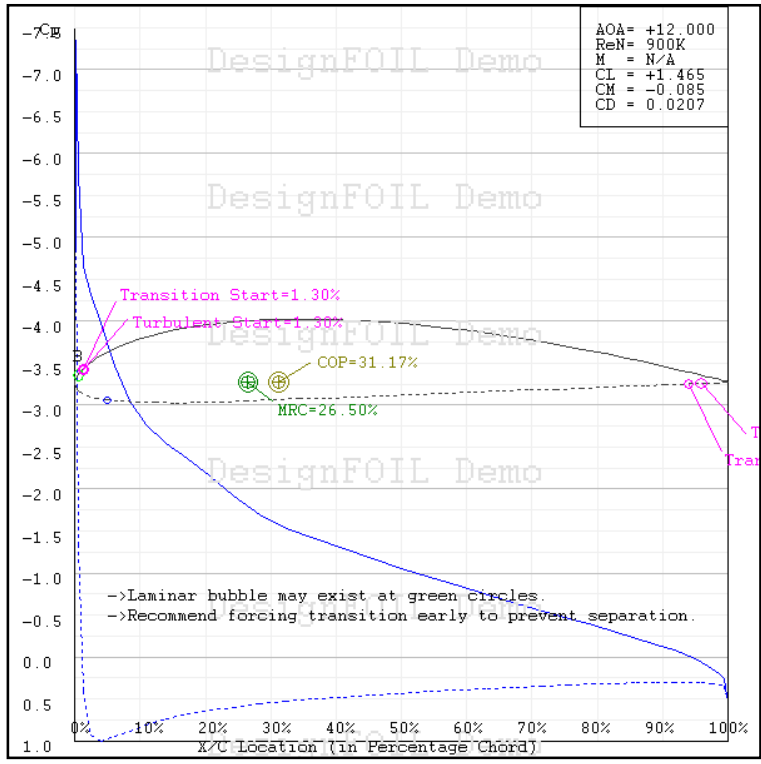

Figure 132. 2-D Airfoil Data at 12 AOA

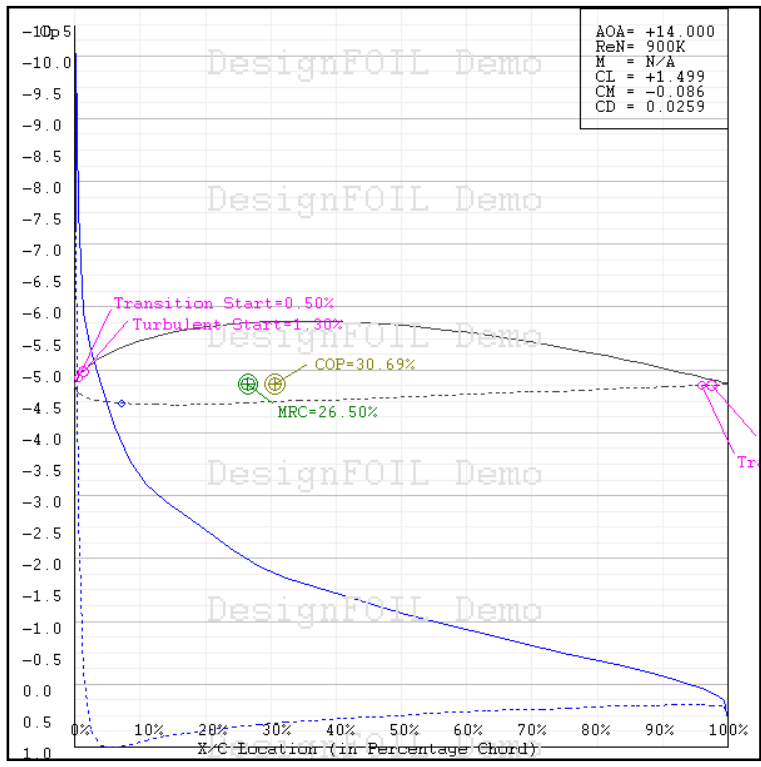

Figure 133. 2-D Airfoil Data at $14 A O A$ 


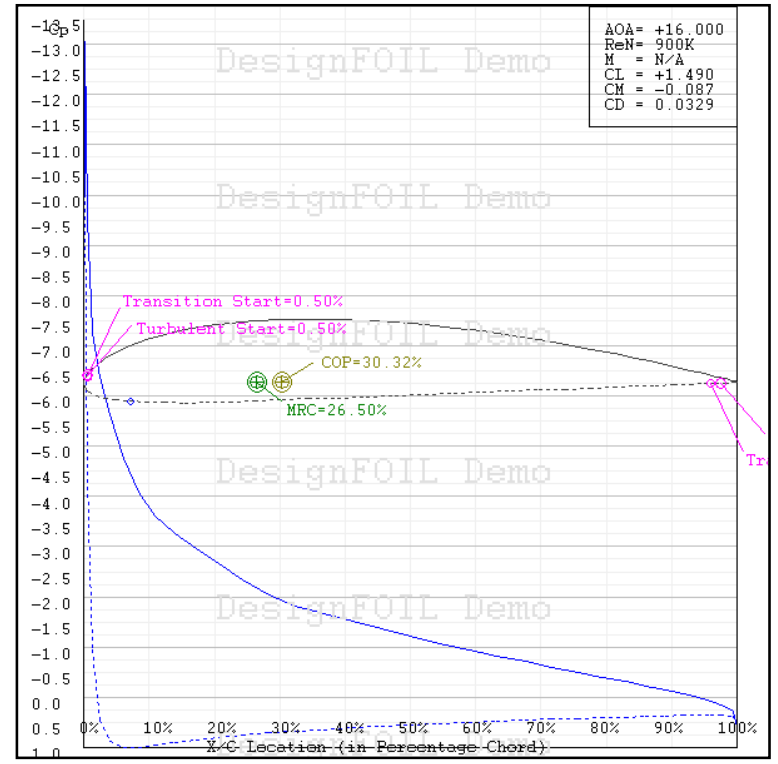

Figure 134. 2-D Airfoil Data at 16 AOA

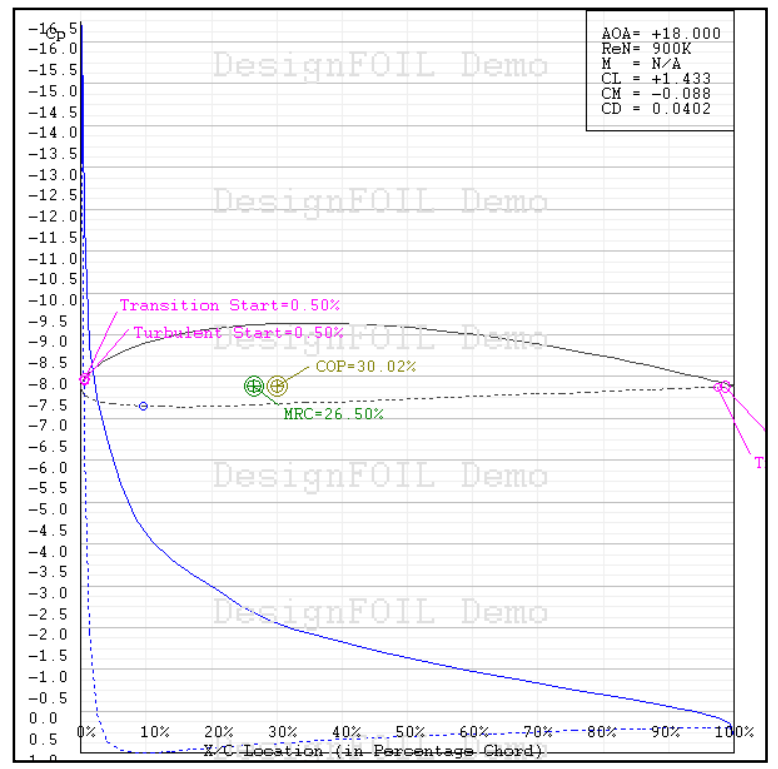

Figure 135. 2-D Airfoil Data at 18 AOA

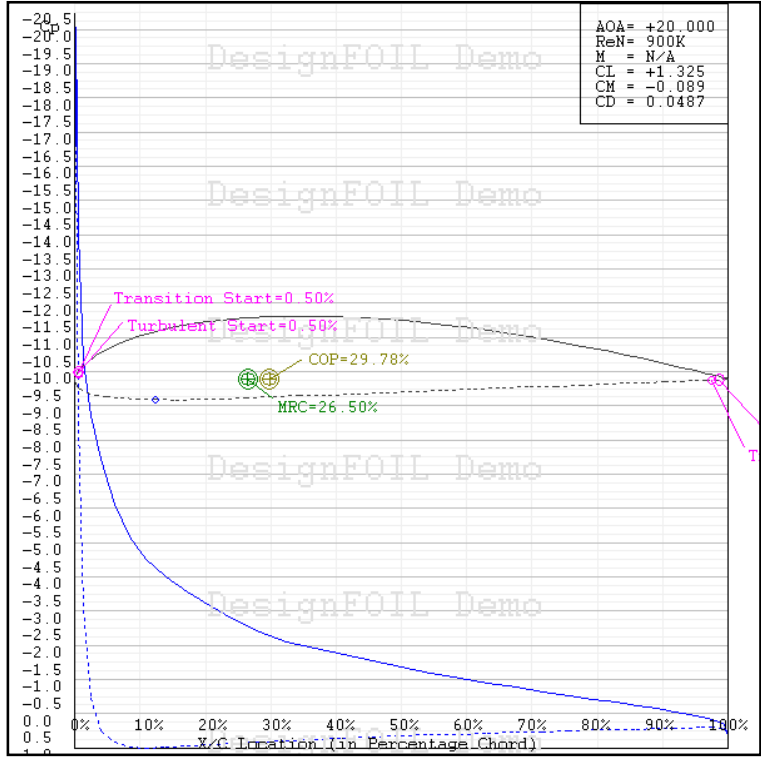

Figure 136. 2-D Airfoil Data at 20 AOA 


\section{Appendix H - Two-Dimensional Clark-Y Airfoil Data}

Result data gathered from the DesignFoil software for a Clark-Y cross-sectioned airfoil, specifically two-dimensional lift and drag profiles swept over angle-of-attack and Reynolds Number, is shown in this section. These results of the section lift and drag coefficient analysis were studied over several Reynolds Numbers and compiled to show their variance, as seen in Figure 137 and Figure 138. As shown, the Reynolds Number variation has little effect on the lift coefficient with respect to the angle-of-attack up to 20.0 degrees. The drag coefficient data however, retains a more significant change across the same range of Reynolds Numbers.

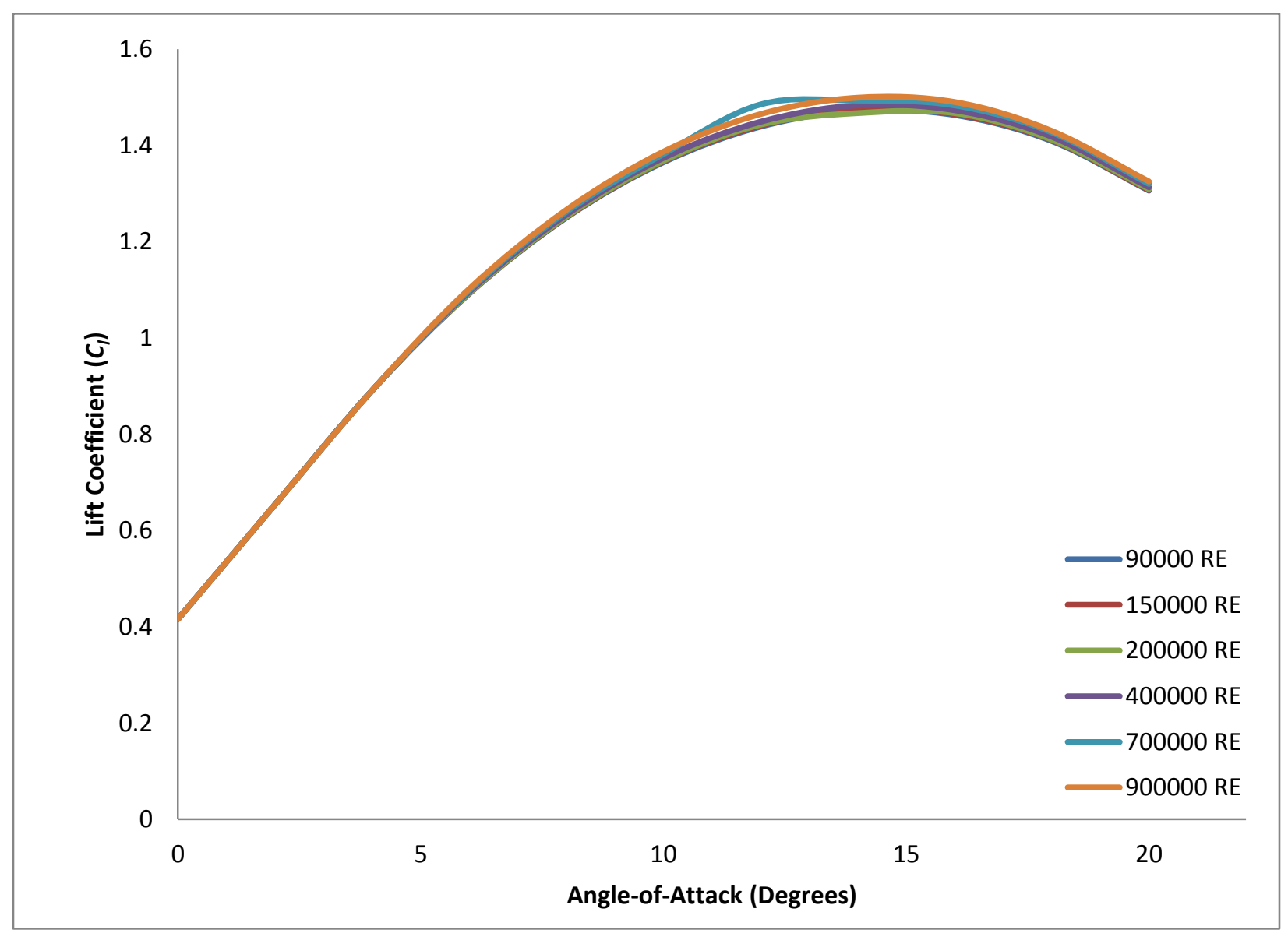

Figure 137. $C_{l}$ vs Angle-of-Attack for Clark-Y Cross-Section 


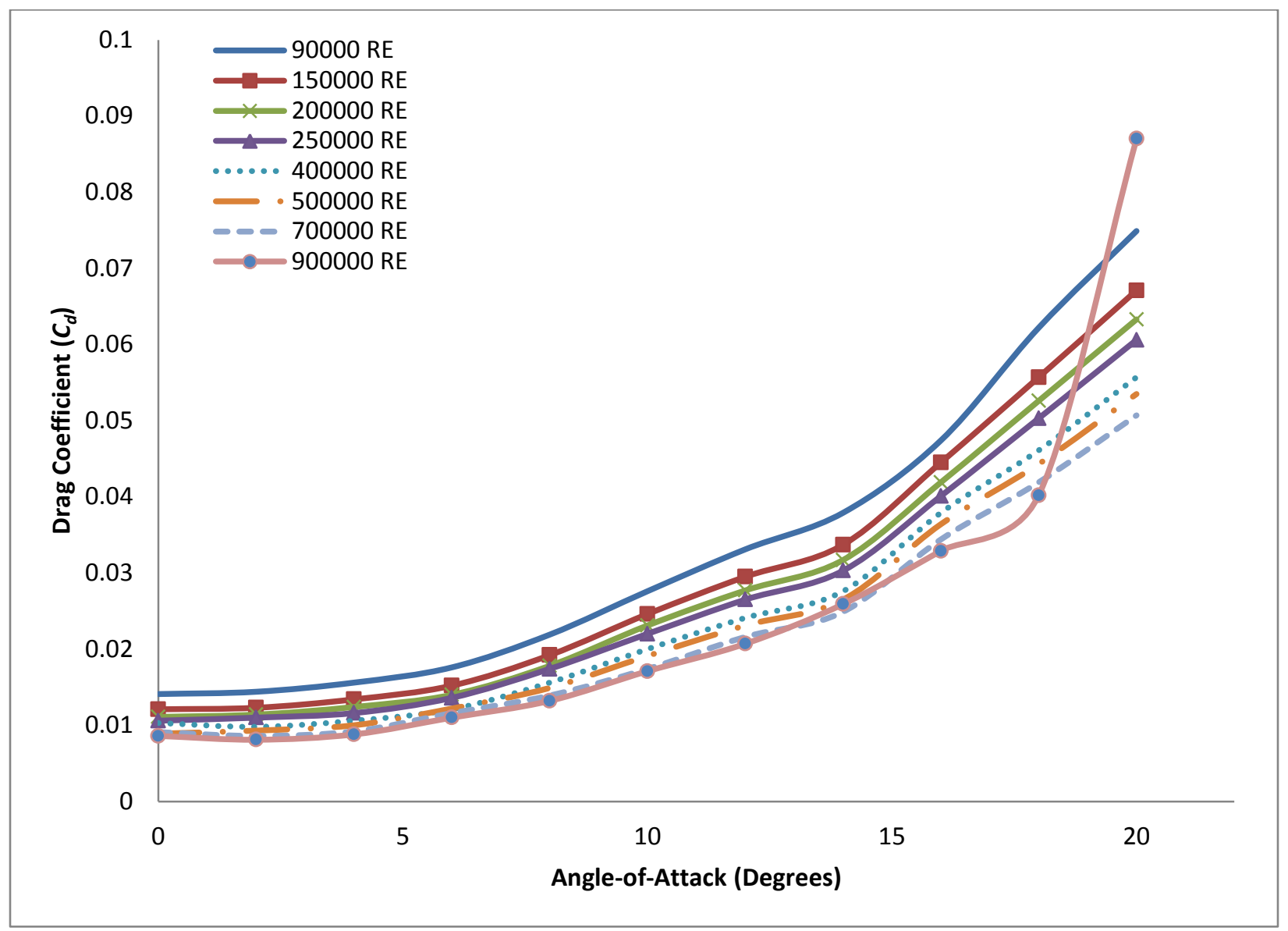

Figure 138. $C_{d}$ vs Angle-of-Attack for Clark-Y Cross-Section 


\section{Appendix I - Experimental Thrust Comparisons}

\section{WVU Experiments}

The following data is given to show the difference between the experimentation data collected from the un-augmented propeller and the active circulation control propeller at various tunnel free-stream velocities. The given values include settings of tunnel fan input of $4.0 \mathrm{~Hz}$, $29.0 \mathrm{~Hz}, 54.0 \mathrm{~Hz}$, and $60.0 \mathrm{~Hz}$, translated to $0.50 \mathrm{~m} / \mathrm{s}, 4.50 \mathrm{~m} / \mathrm{s}, 9.50 \mathrm{~m} / \mathrm{s}$, and $10.50 \mathrm{~m} / \mathrm{s}$ freestream velocity into the propeller plane, respectively. Figure 139, Figure 140, Figure 141, and Figure 142 below show the results of this experimentation, and show that at advance ratios between 0.10 and 0.40 the output thrust of the augmented propeller is greater than that of the baseline un-augmented propeller, in some cases by 2.0-3.0 percent.

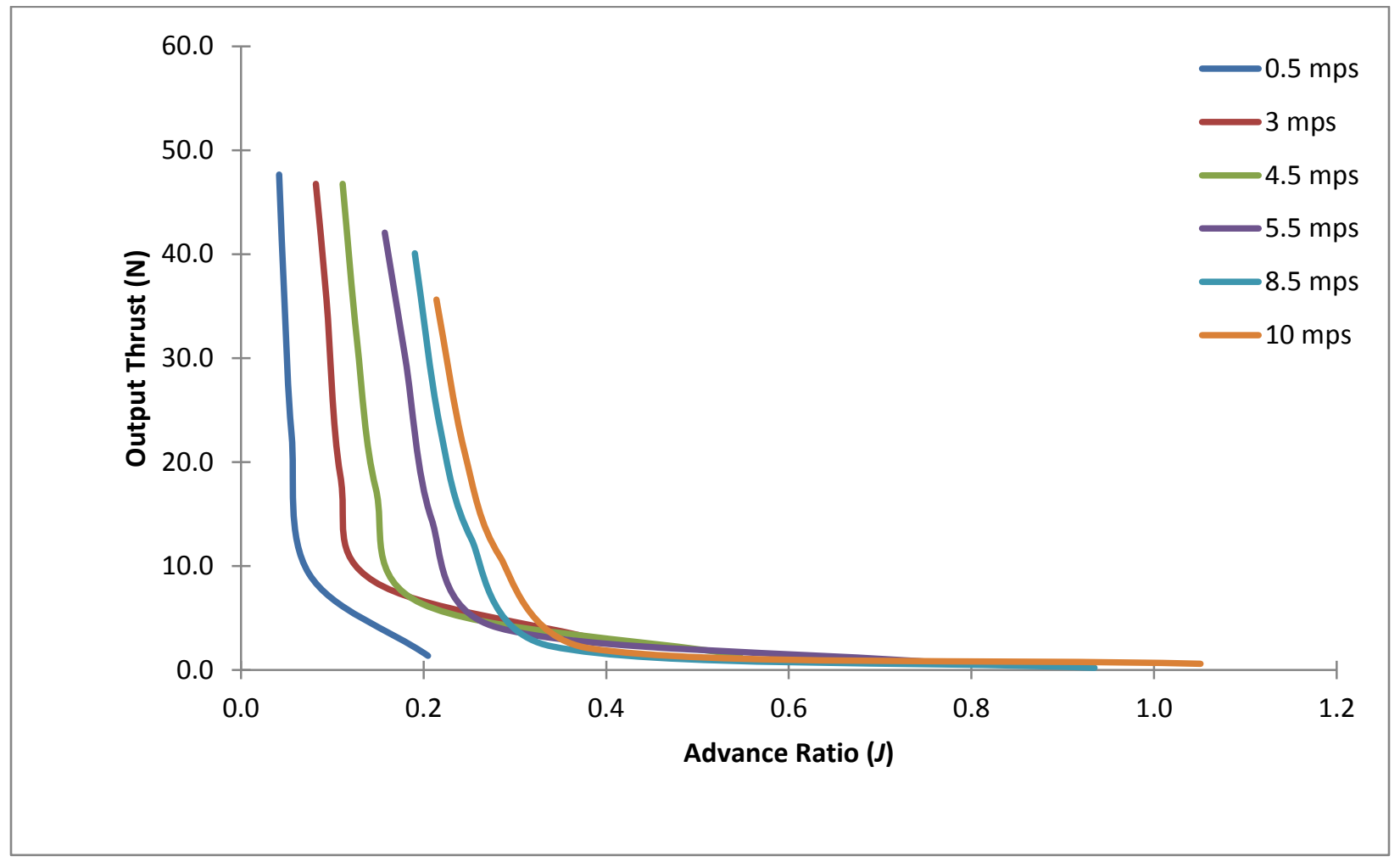

Figure 139. Output Thrust versus Advance Ratio at Tunnel Speed (Unaugmented Prop) 


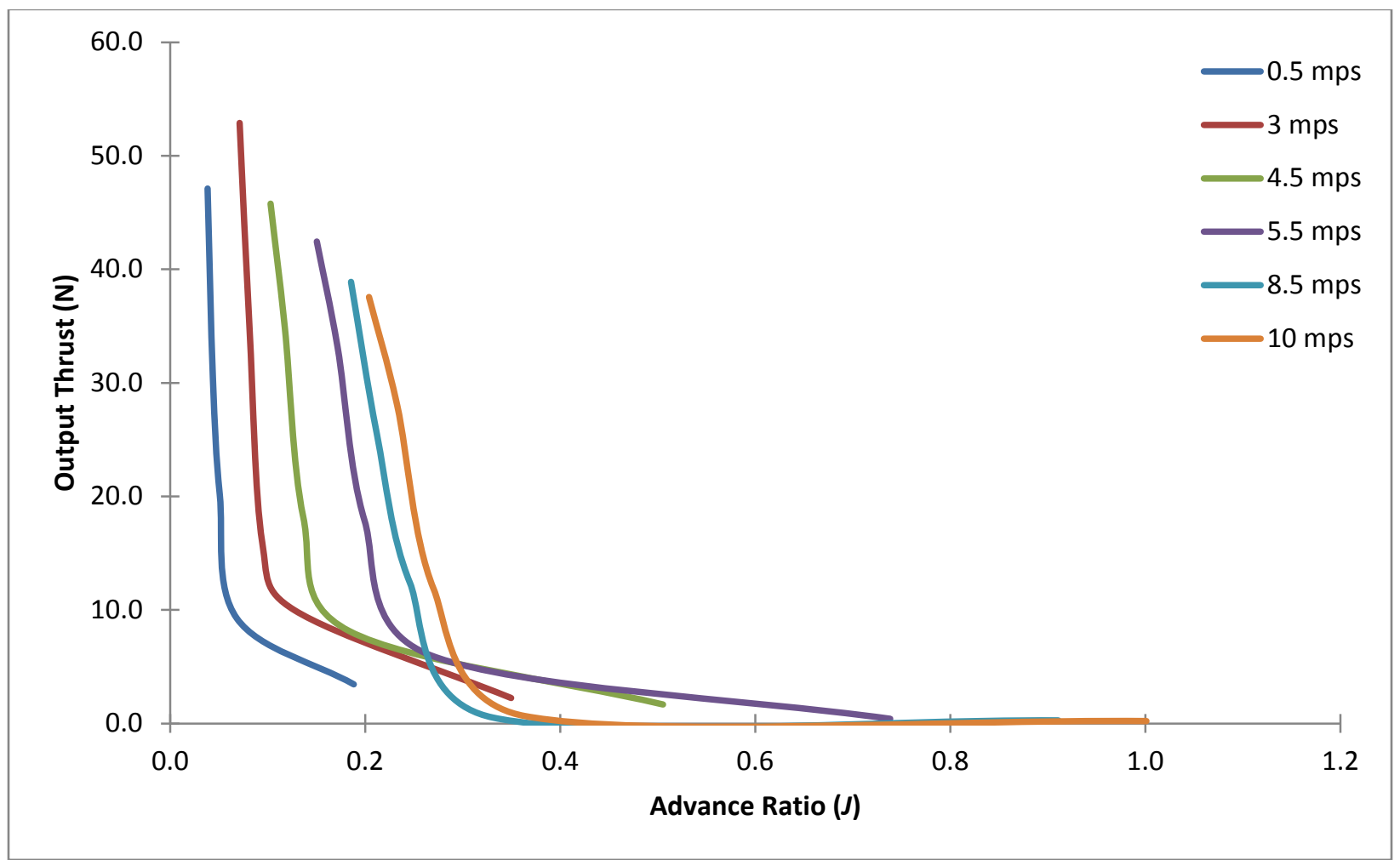

Figure 140. Output Thrust Versus Advance Ratio at Tunnel Speed (Augmented Prop)

\section{WPAFB Experiments}

This section documents the thrust capabilities measure between the unaugmented and augmented propellers as a function of advance ratio tested in both the $W V U$ wind tunnel and the $W P A F B$ wind tunnel, in two different experimental studies. 


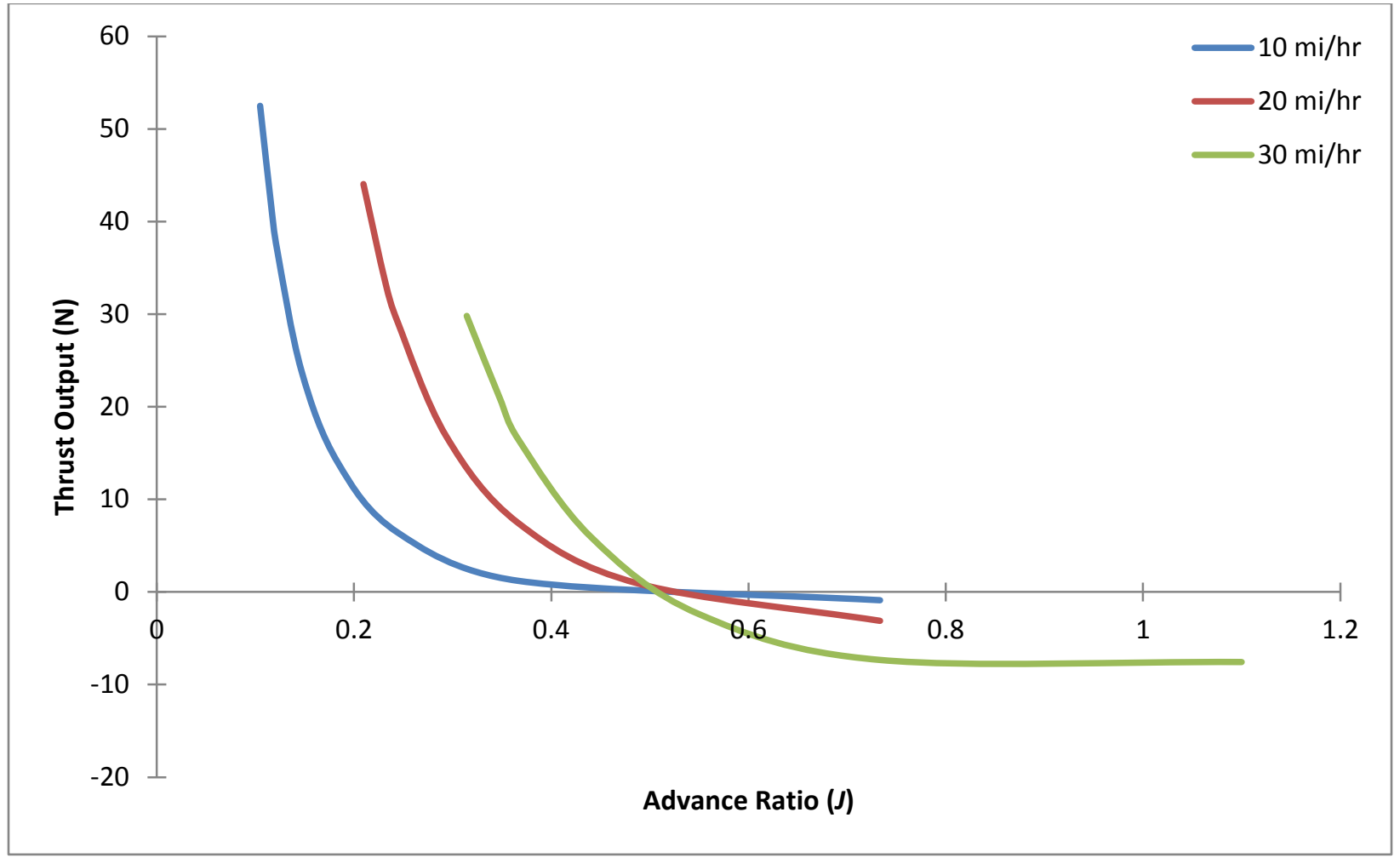

Figure 141. Output Thrust versus Advance Ratio at Tunnel Speed (Unaugmented Prop)

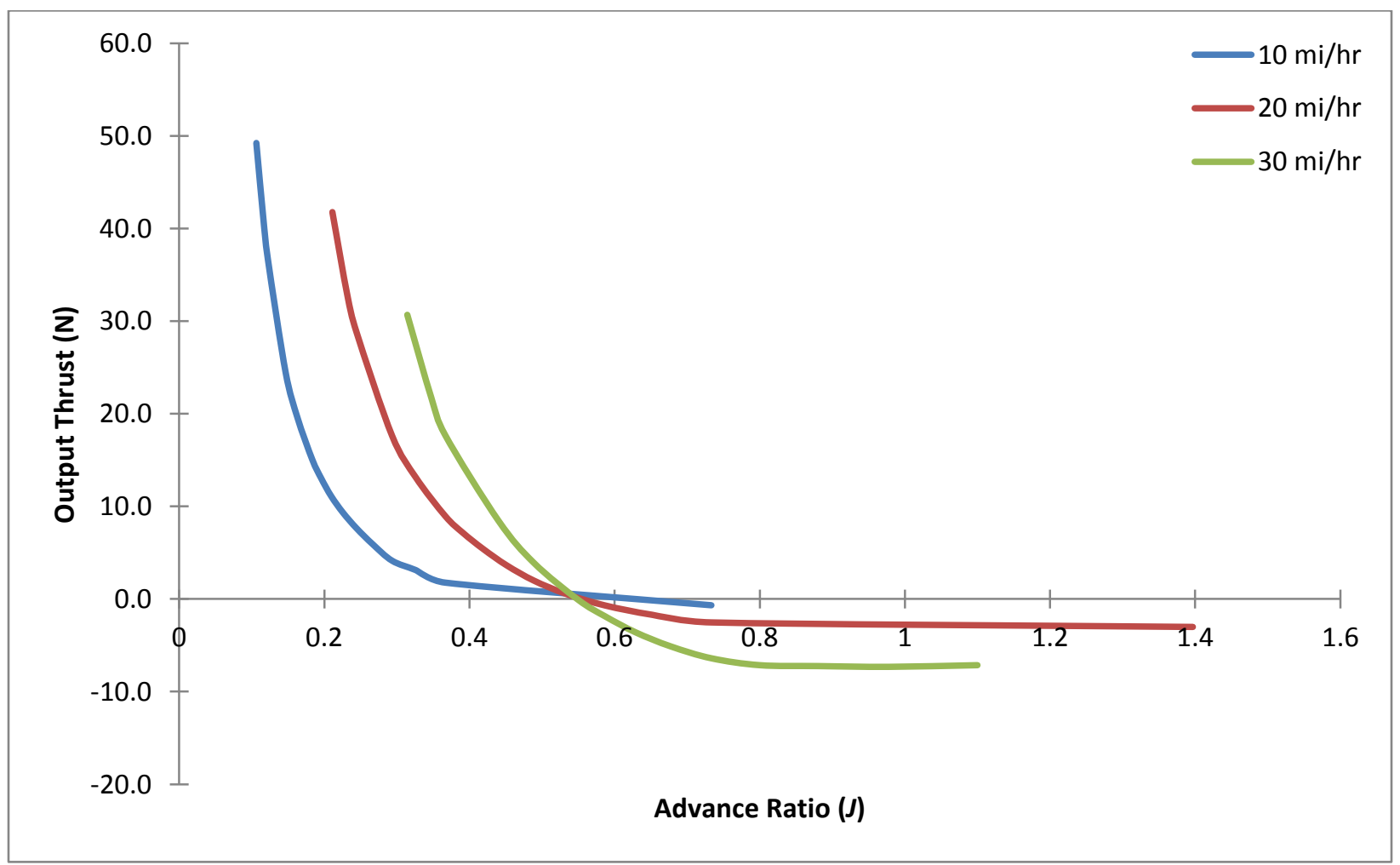

Figure 142. Output Thrust versus Advance Ratio at Tunnel Speed (Augmented Prop) 


\section{Appendix J - Experimental Torque Comparisons}

\section{WVU Experiments}

The following data is given to show the difference between the input torque required to turn both the un-augmented propeller and the active circulation control propeller at various tunnel free-stream velocities during experimentation. The given values include settings of tunnel fan input of $4.0 \mathrm{~Hz}, 29.0 \mathrm{~Hz}, 54.0 \mathrm{~Hz}$, and $60.0 \mathrm{~Hz}$, translated to $0.50 \mathrm{~m} / \mathrm{s}, 4.50 \mathrm{~m} / \mathrm{s}, 8.50 \mathrm{~m} / \mathrm{s}$, and $10.0 \mathrm{~m} / \mathrm{s}$ free-stream velocity into the propeller plane, respectively. Figure 143, Figure 144, Figure 145, and Figure 146 below, show the results of the experimental trials conducted while monitoring the effect that adding circulation control had on torque required on the system. In all the cases studied, the input torque at a given advance ratio was measure to be equal or less on the augmented propeller than on the baseline un-augmented propeller studies. In some situations, the input torque was shown to be approximately 40.0 percent less when results were compared side by side, showing an effective decrease in overall pressure drag on the blades. This torque input reduction, coupled with the area of effective thrust force increase, the efficiency factor of the propeller increases over this optimal range of advance ratios $(J)$, which experimentally spans $J$ values of 0.10 to 0.40 . 


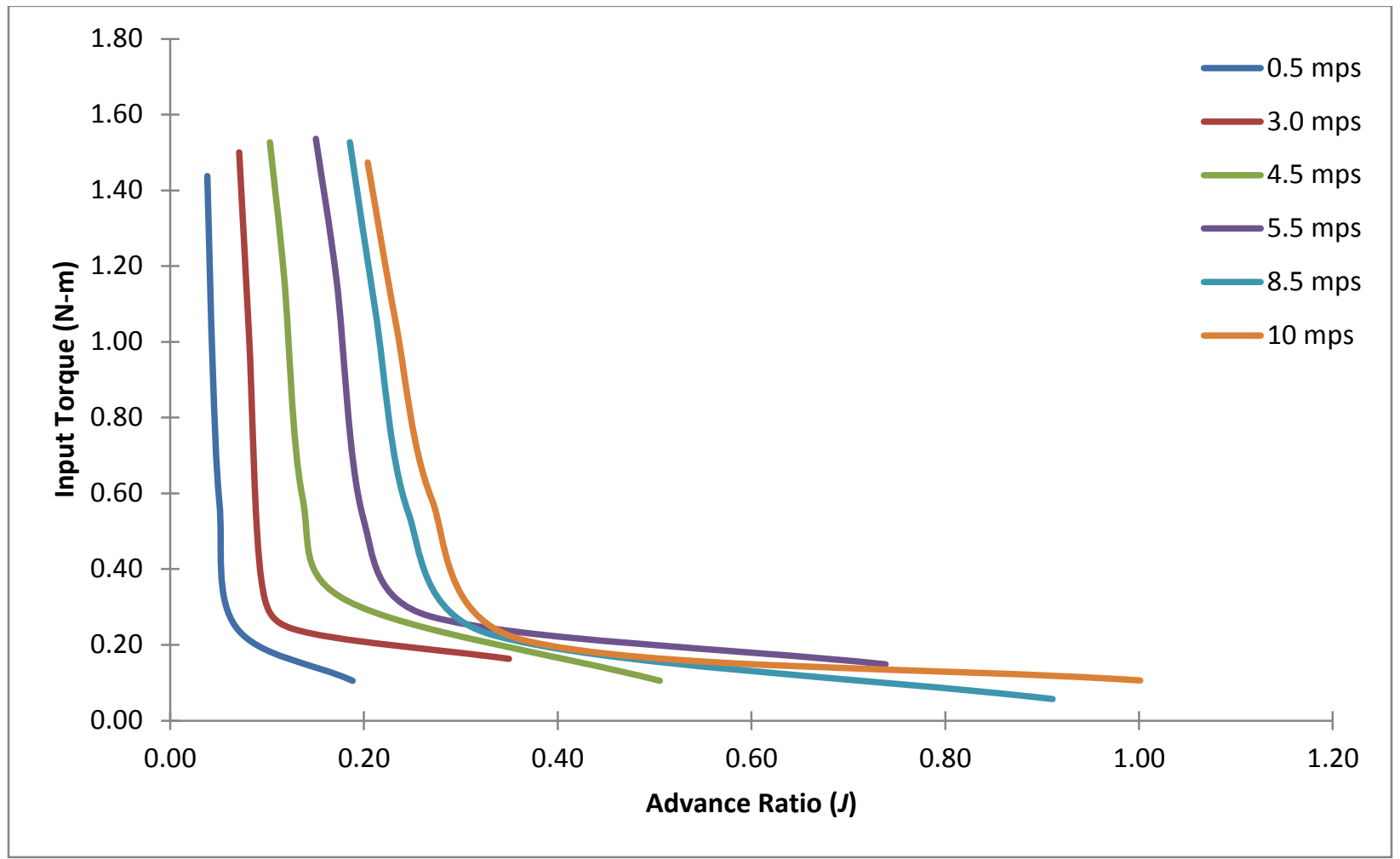

Figure 143. Input Torque Versus Advance Ratio at Tunnel Speed (Unaugmented Prop)

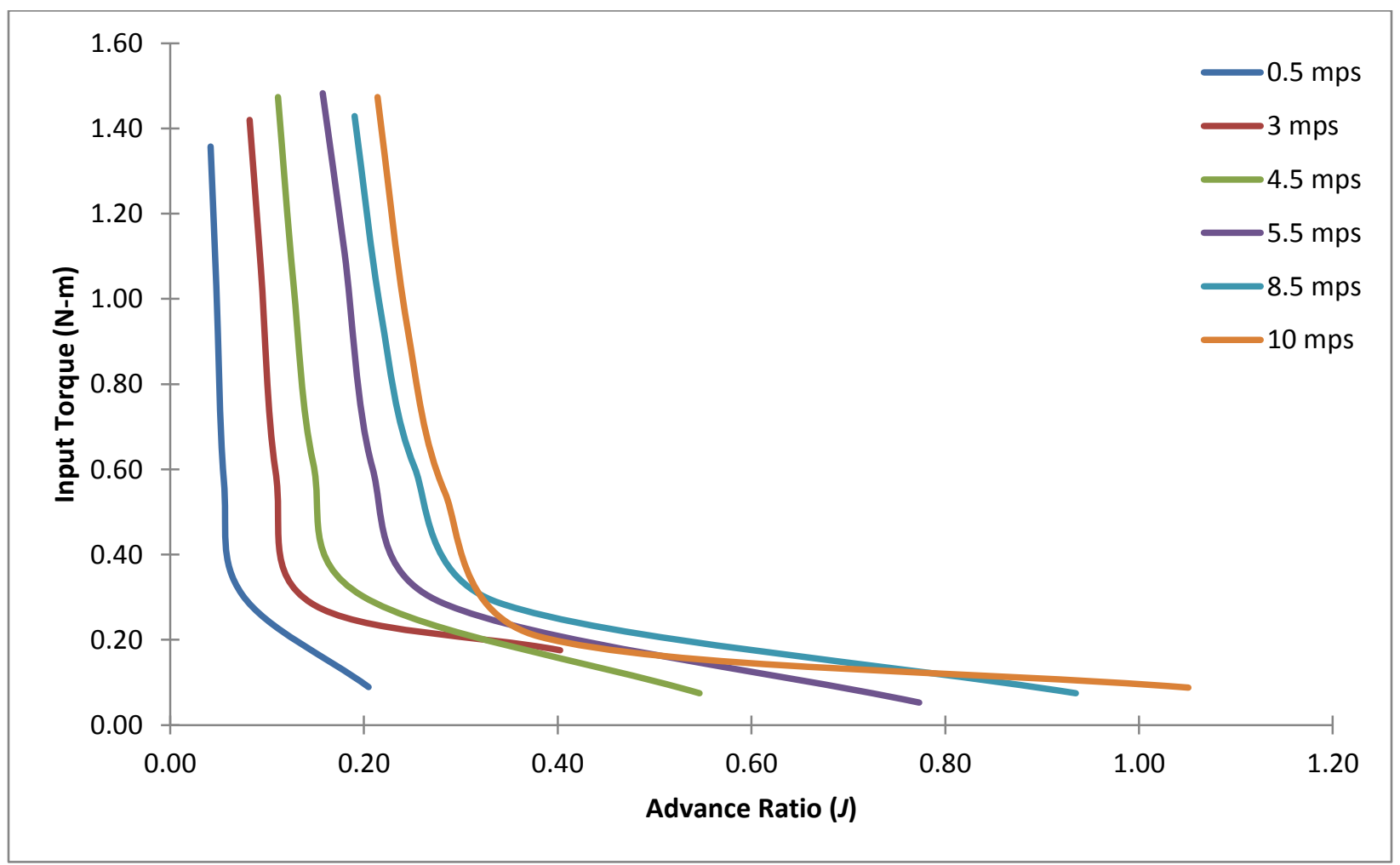

Figure 144. Input Torque Versus Advance Ratio at Tunnel Speed (Augmented Prop) 


\section{WPAFB Experiments}

This section documents the thrust capabilities measure between the unaugmented and augmented propellers as a function of advance ratio tested in both the $W V U$ wind tunnel and the $W P A F B$ wind tunnel, in two different experimental studies. These experiments ranged over a variety of tunnel speeds $(10.0,20.0$, and 30.0 miles per hour) as well as rotational speeds, providing the remaining information needed to estimate a full propeller performance prediction.

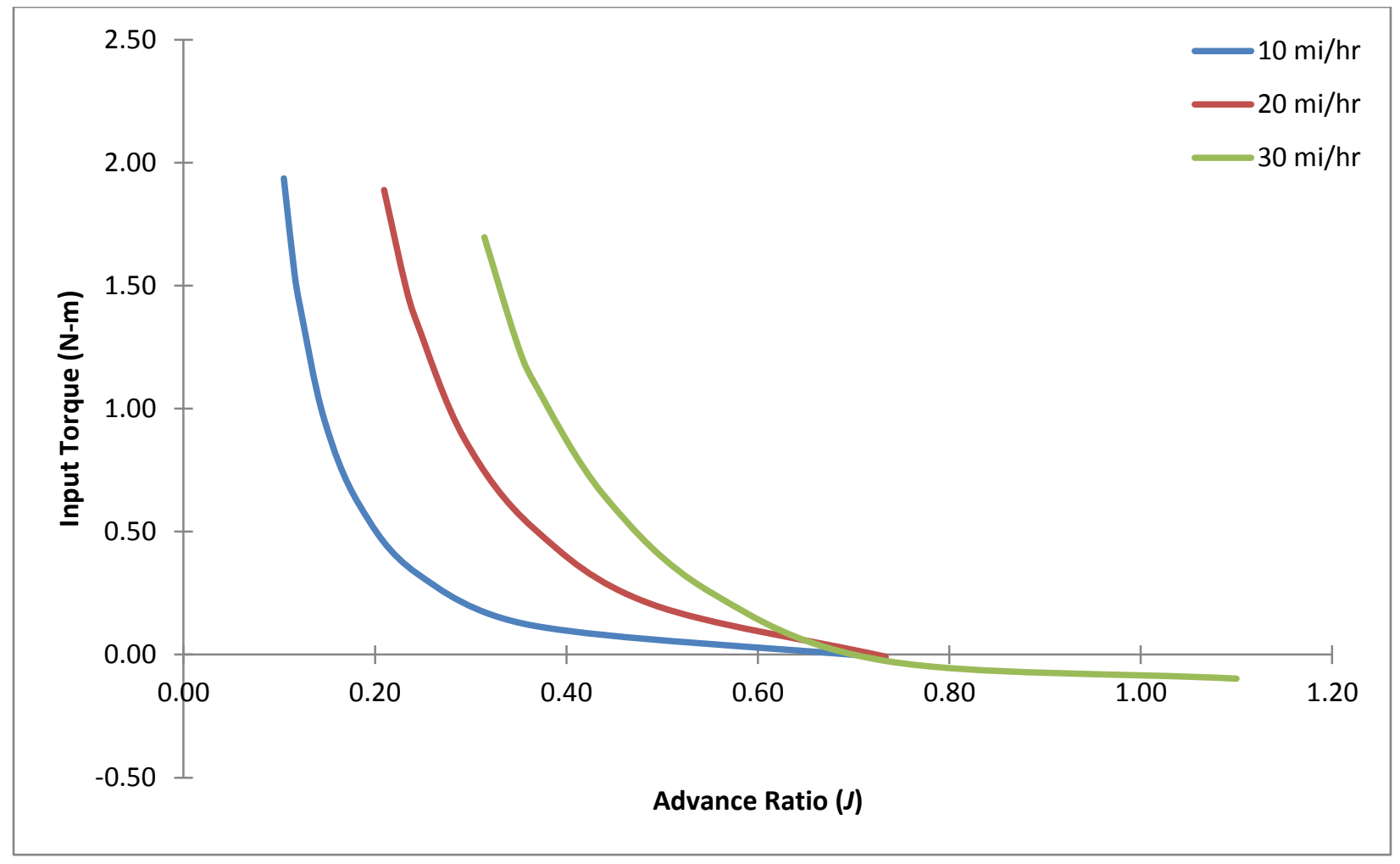

Figure 145. Input Torque with Respect to Advance Ratio Over Tunnel Speed (Unaugmented Prop) 


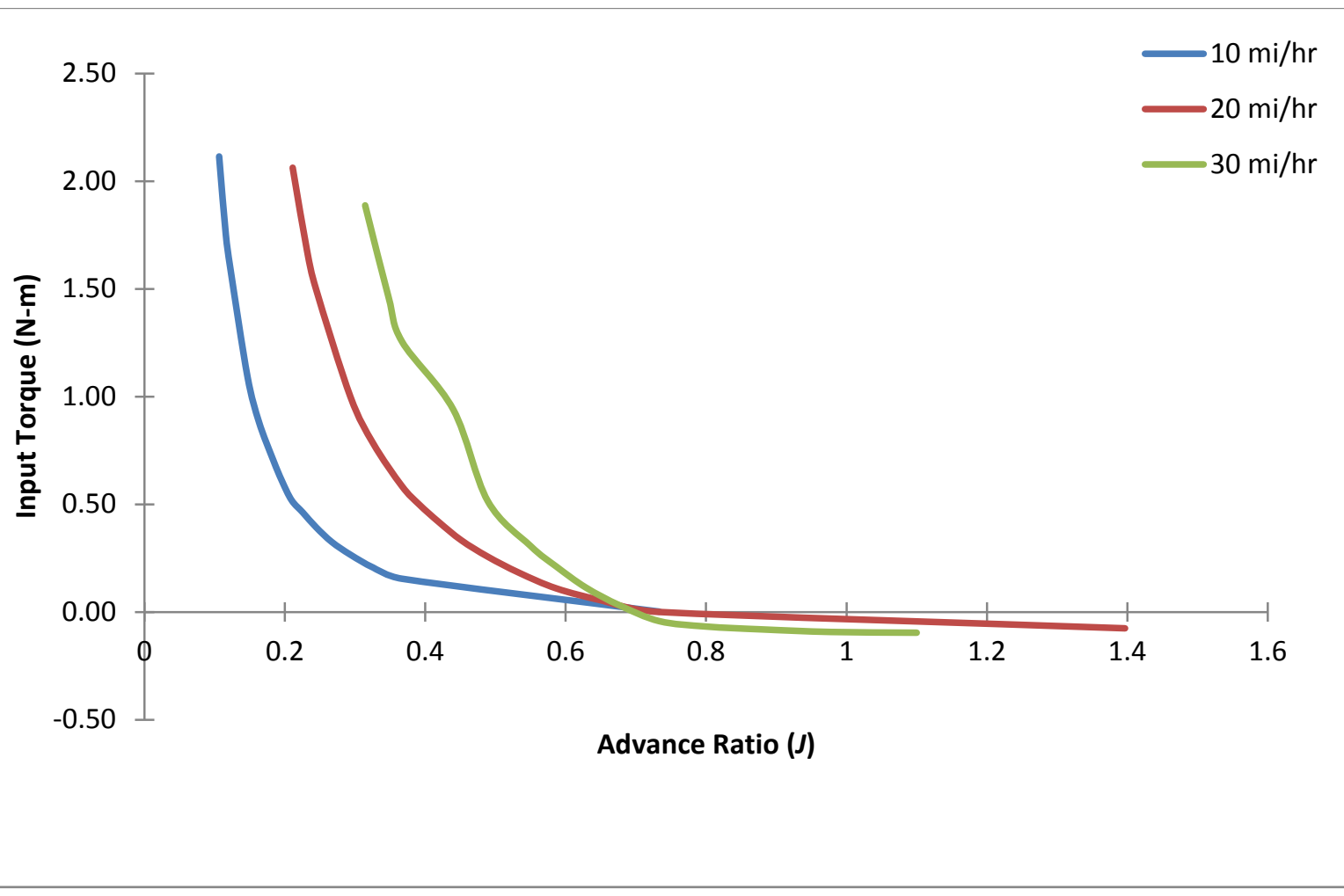

Figure 146. Input Torque with Respect to Advance Ratio Over Tunnel Speed (Augmented Prop) 


\section{Appendix K - Raw Data from Wind Tunnel Experiments}

This appendix sections serves to illustrate the comparison of data between the WrightPatterson Air Force Base Vertical Wind Tunnel experiments as well as the experiments performed at $W V U$. Each of the comparisons contained in this appendix, shows unaugmented propeller data compared with augmented data collected, and includes data from both experimental facilities. Each of the plots are based on coefficient data rendering the resulting data non-dimensional, and comparable to any propeller in any experimental situation. Corresponding analytical predictions and curve fit tend lines are also shown for each set of data.

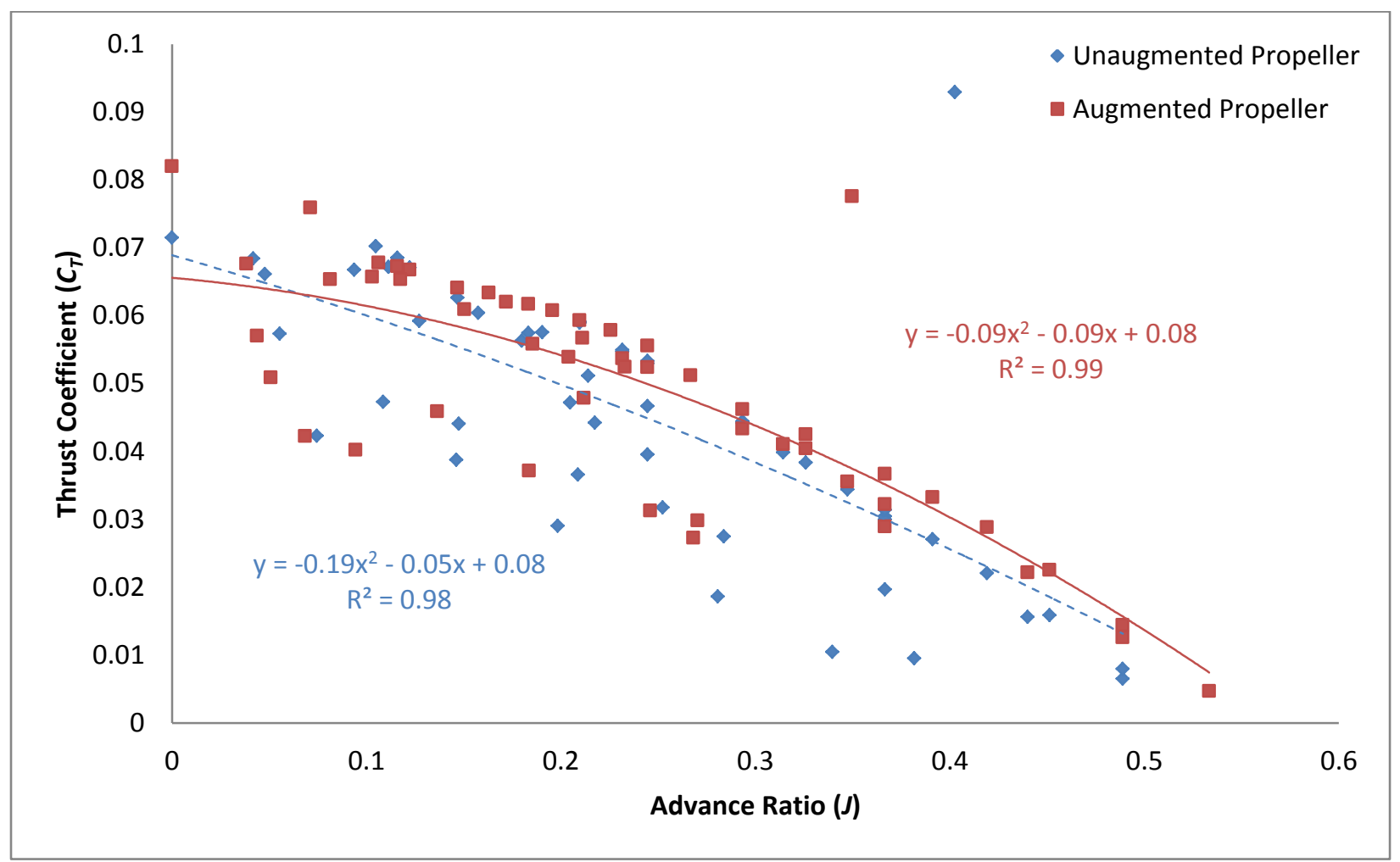

Figure 147. Thrust Coefficient versus Advance Ratio 


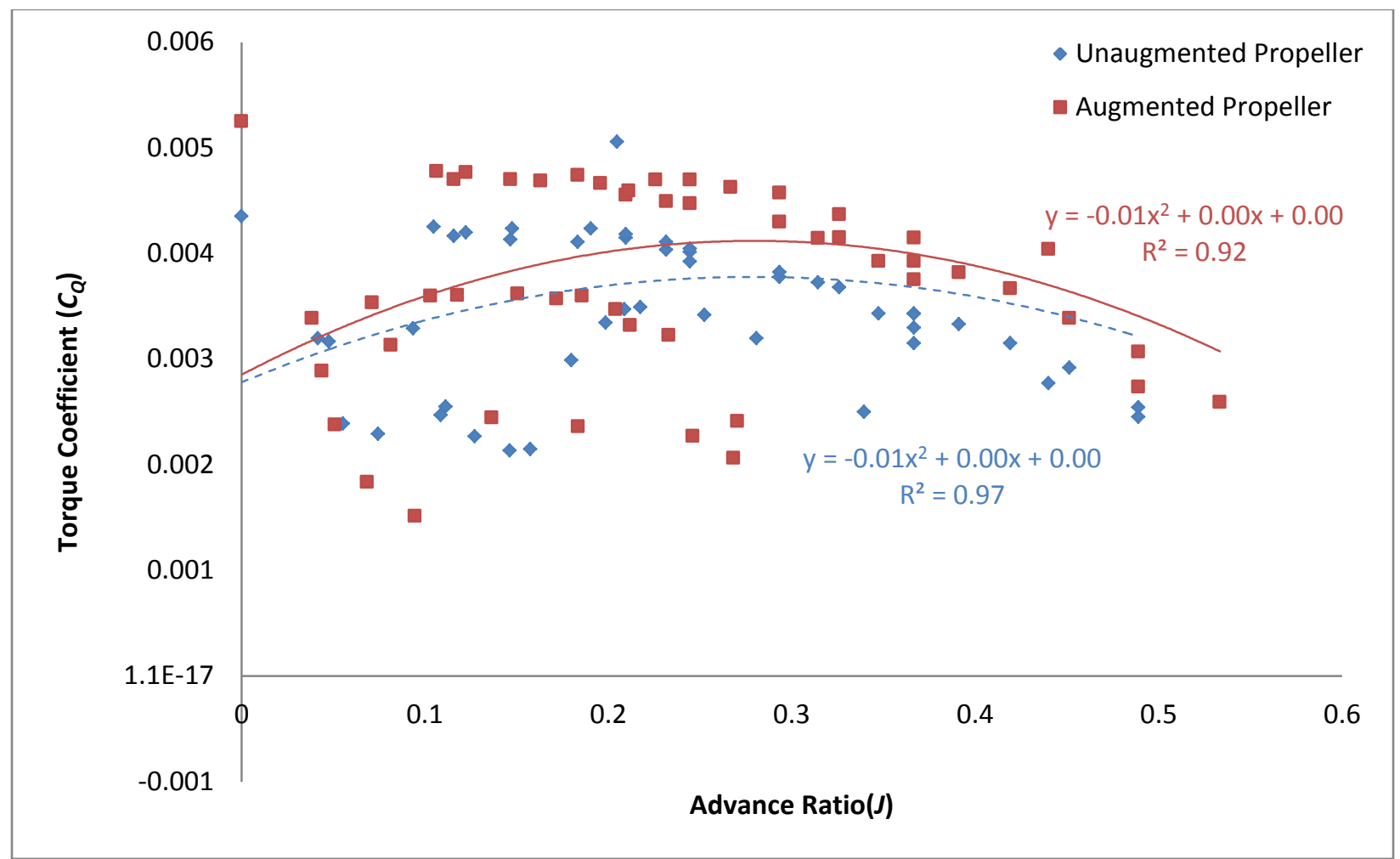

Figure 148. Coffecient of Torque versus Advance Ratio of Experimental Propellers

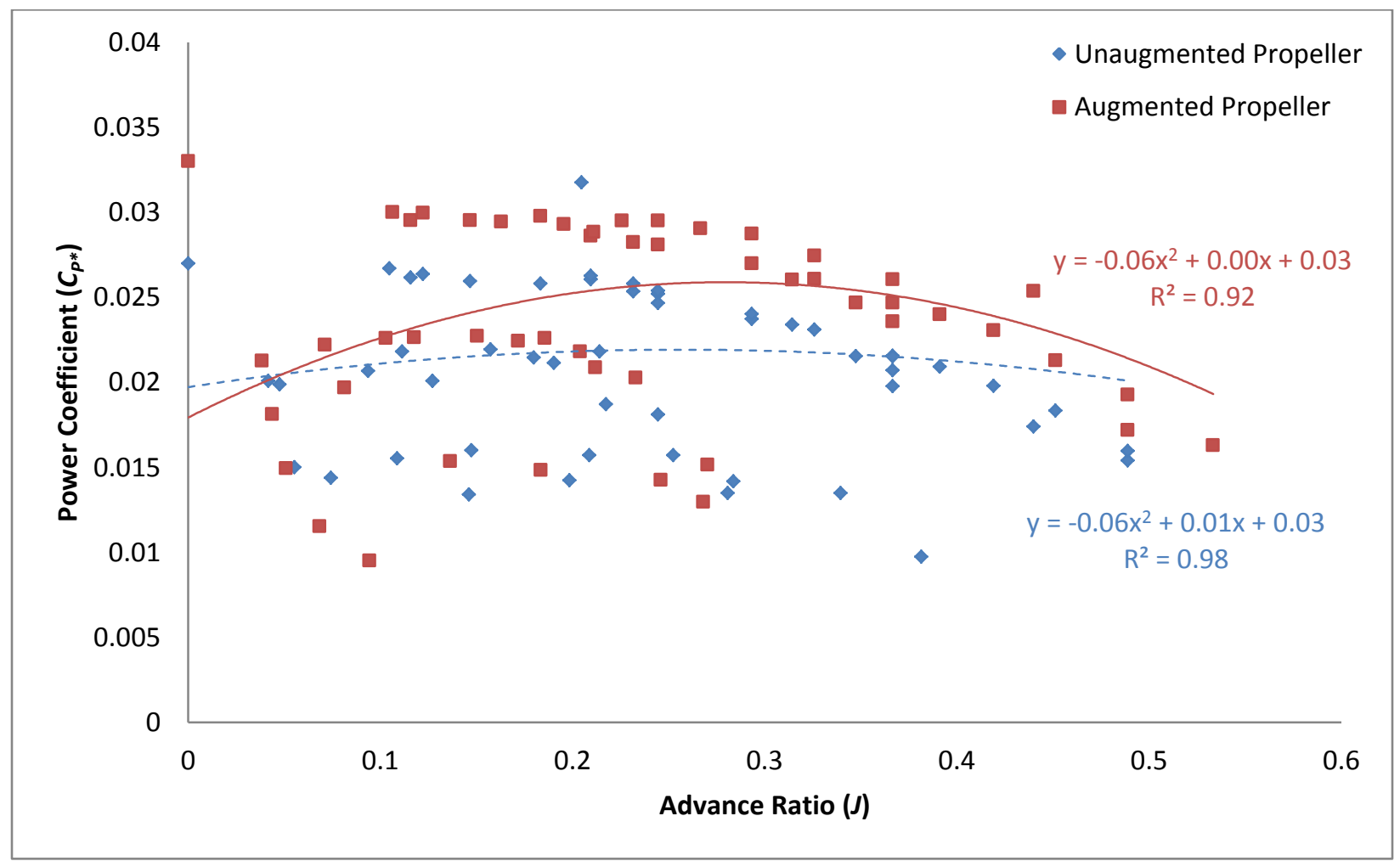

Figure 149. Propeller Power Coefficient versus Advance Ratio 


\section{Appendix L - Castle Link Speed Controller Data Logger}

This section documents the output files of the data logger used while testing in the $W P A F B$ vertical wind tunnel. The small device, made by Castle Creations, was attached to the $E S C$ and was able to correlate input power, voltage, current, and rotational speed of the motor to a time values over the course of the entire experimental test run. This was used a backup for the tunnel data acquisition system and provided a second way of measuring the rotational speed of the motor and propeller. This mainly acted as a checking mechanism to make sure the tunnel data acquisition system was running properly throughout the entire experiment.

\section{Logged Data from WPAFB Experiment - Unaugmented Propeller}

Information follows in the gathered data from ESC input parameters during experimentation with the unaugmented propeller tests conducted at WPAFB. The data served as backup information for the experiments run with the Zinger Inc. propeller at testing conditions and effectively saved, and plotted input rotational speeds, voltage, current and power draw for all the experimental conditions as a function of a time variable. Along with serving and a second set of data acquisition, this also served as an end of run check to make sure all the other components were working properly without the need to download and reduce all the data before moving on to a new data set. 


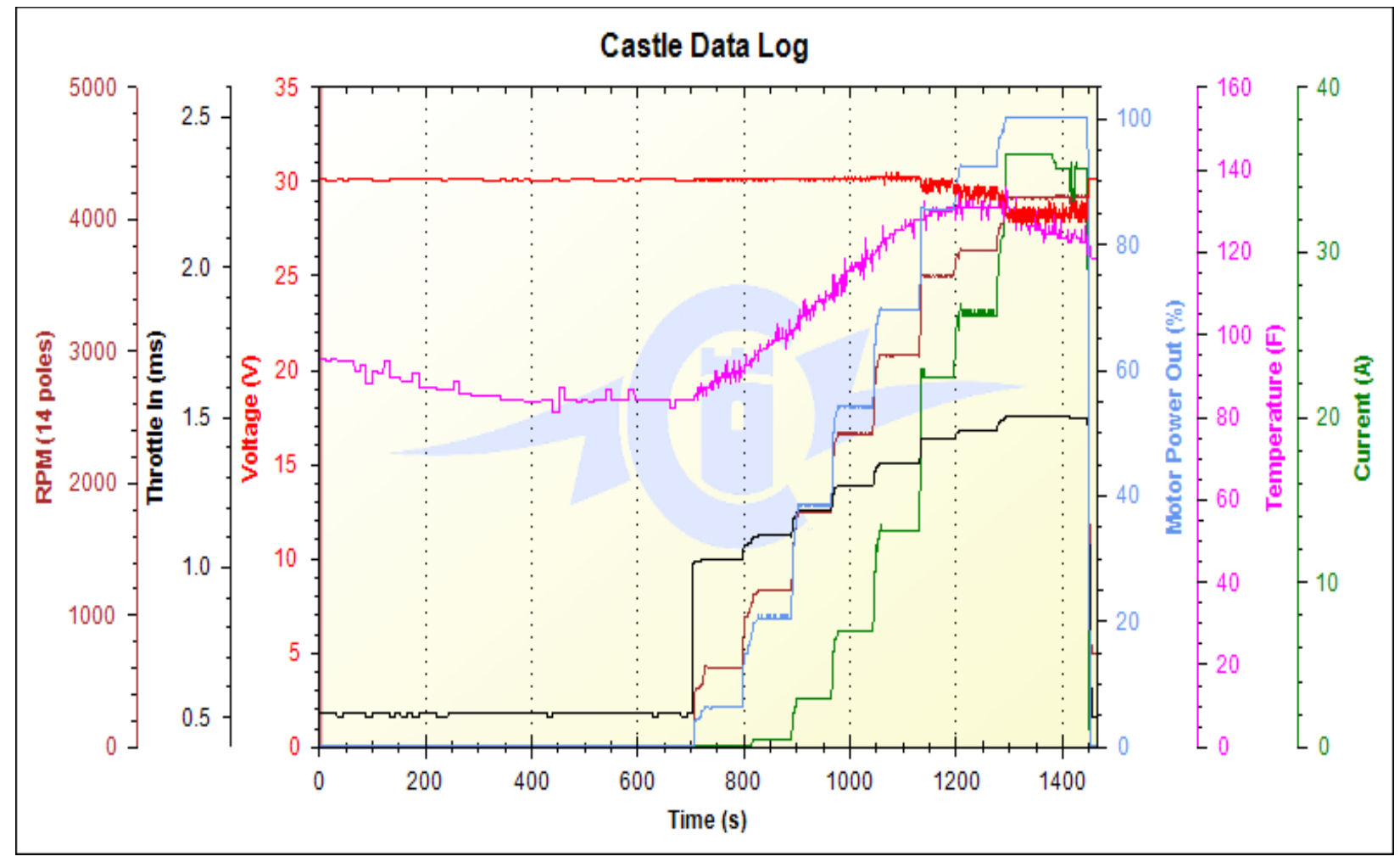

Figure 150. ESC Data at Tunnel Velocity of 10 Miles per Hour

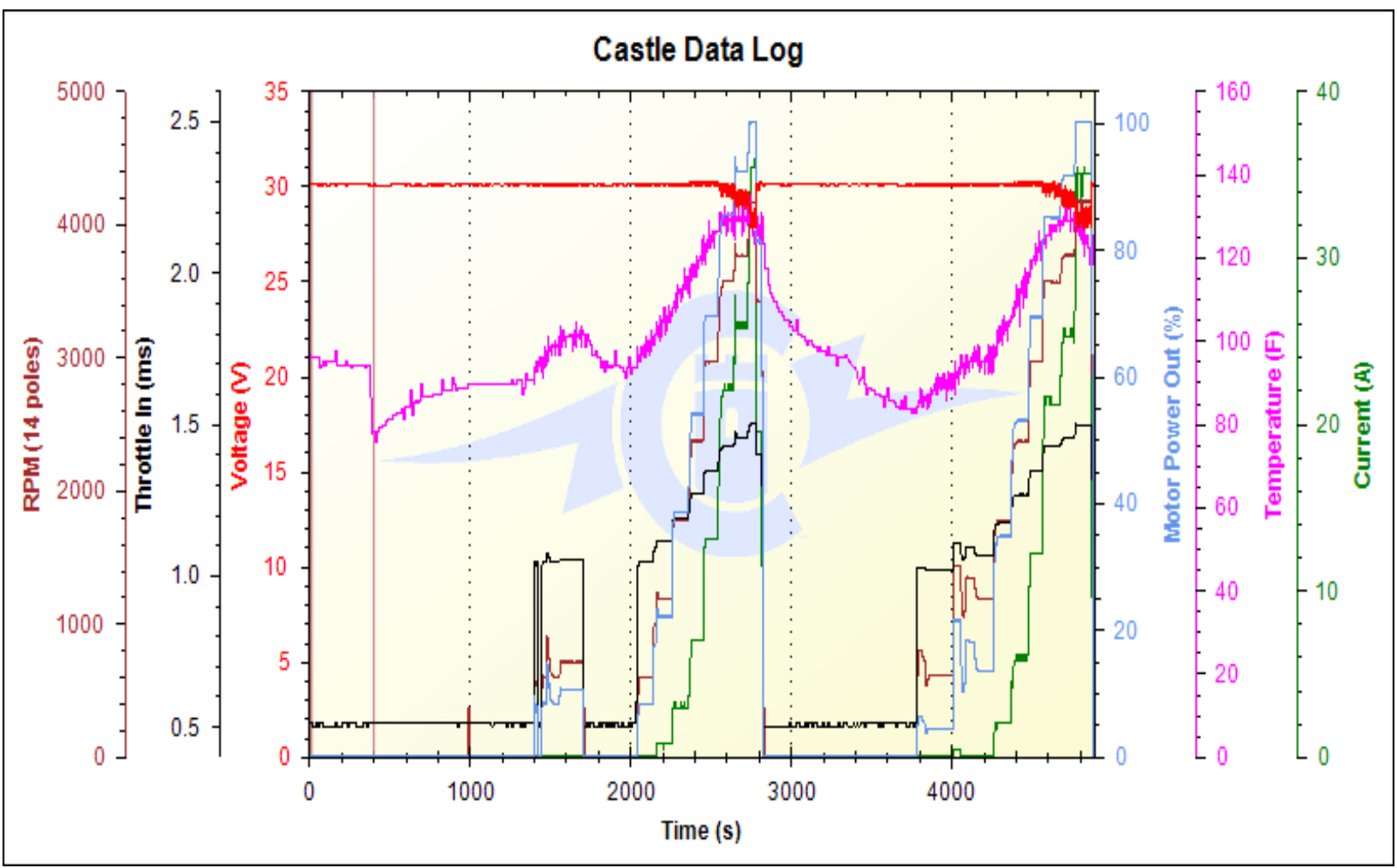

Figure 151. ESC Data at Tunnel Velocity of 20 Miles per Hour 


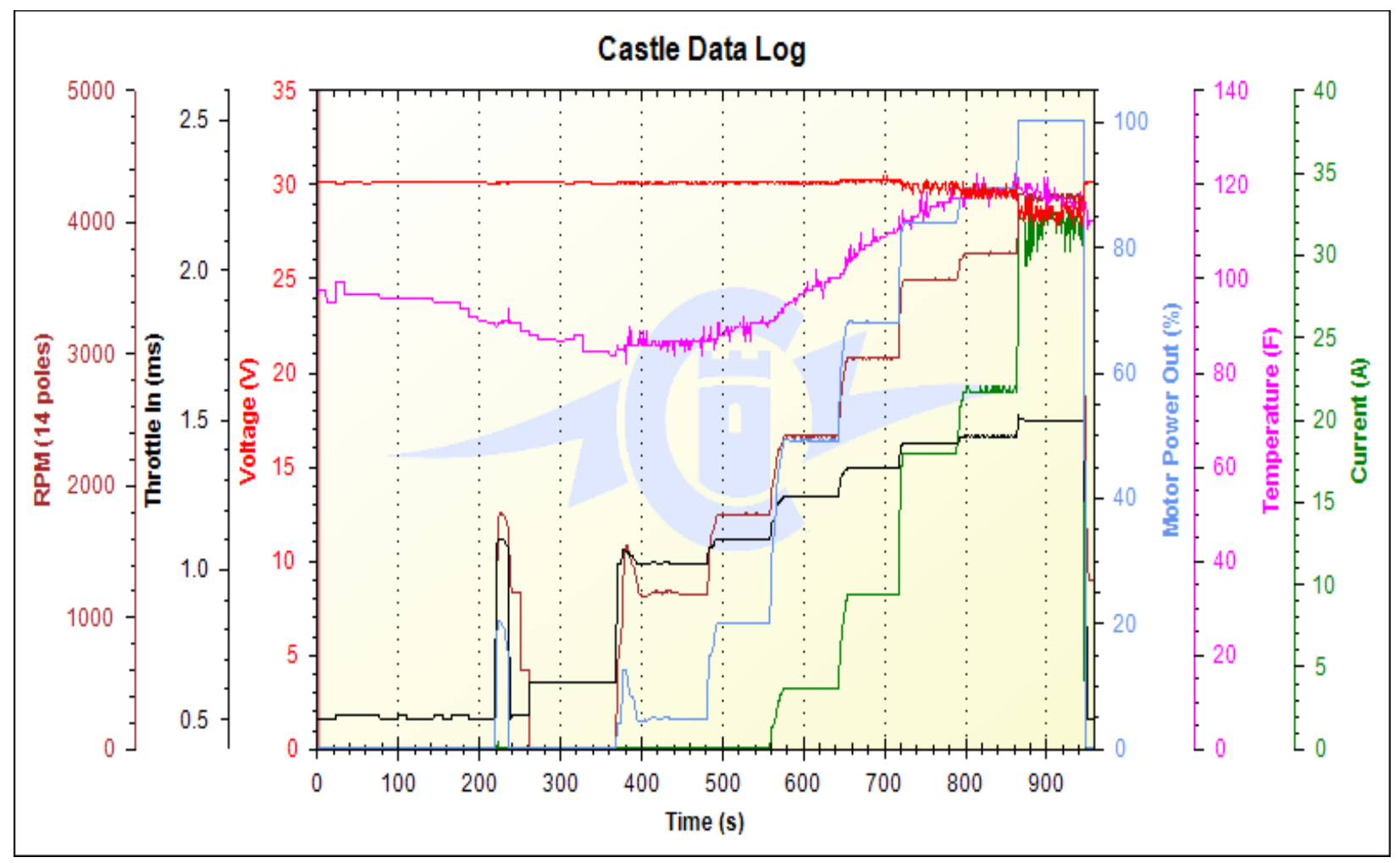

Figure 152. ESC Data at Tunnel Velocity of 30 Miles per Hour 


\section{Logged Data from WPAFB Experiment - Augmented Propeller}

Again similar data was saved from the speed controller with respect to input rotational speeds, temperature, voltage, current and input power as it related to the testing scenarios at the $W P A F B$ wind tunnel. This acted as a back-up set of data for the data acquisition system used while testing the propeller at advance ratios. The following plots (Figure 153, Figure 154, and Figure 155) show the information gathered for the augmented propeller while experimentation was conducted.

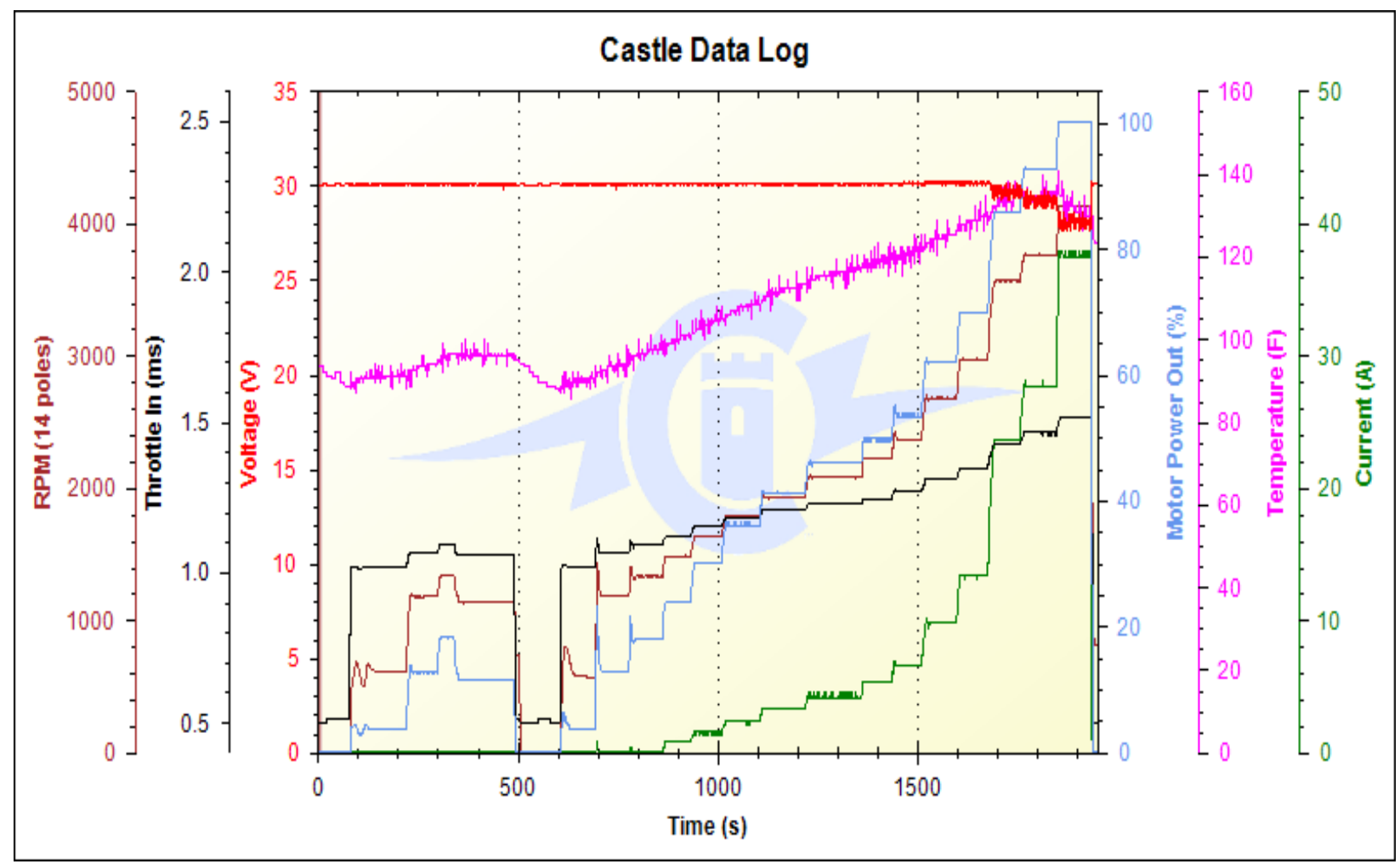

Figure 153. ESC Data at Tunnel Velocity of 10 Miles per Hour 


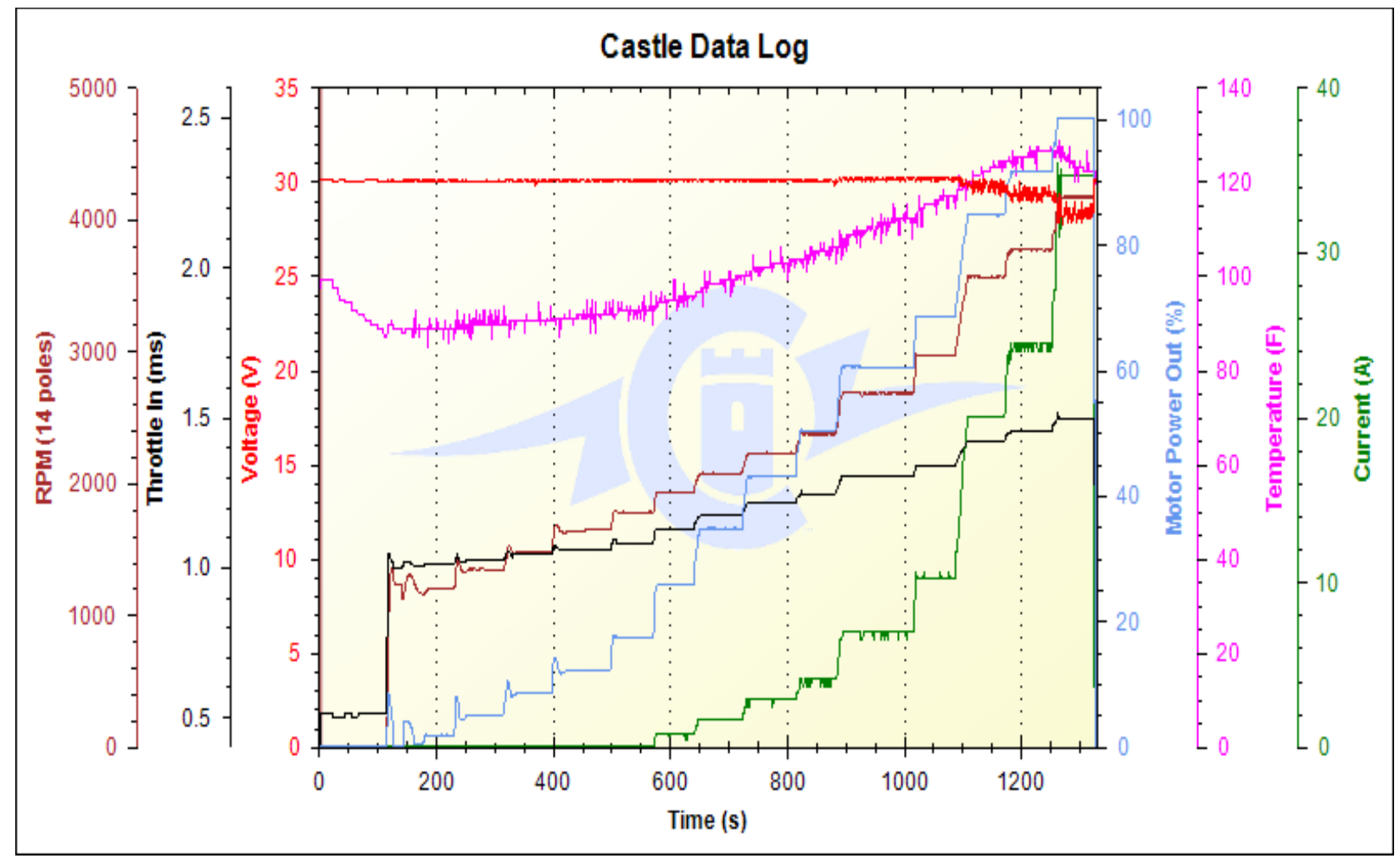

Figure 154. ESC Data at Tunnel Velocity of 20 Miles per Hour

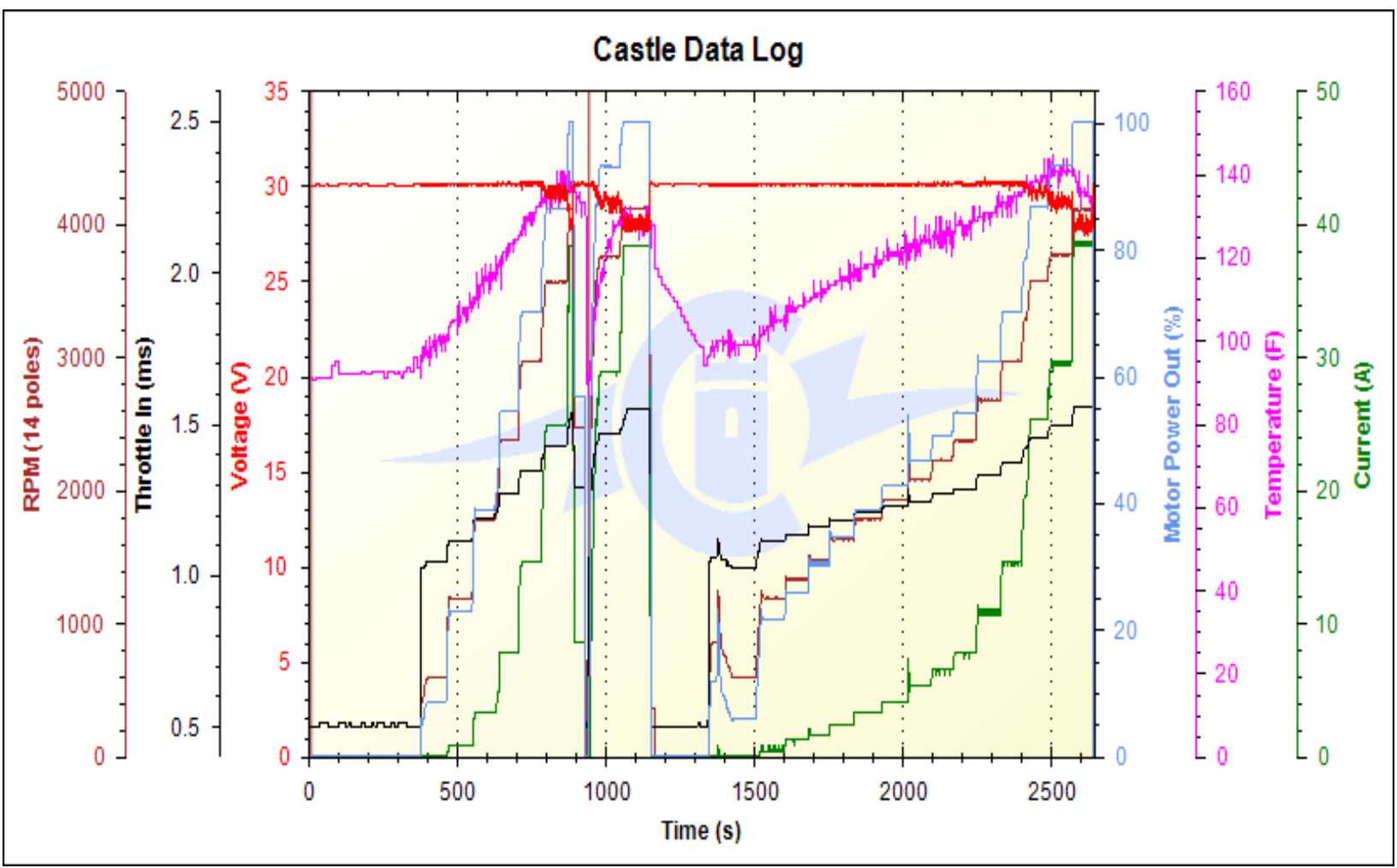

Figure 155. ESC Data at Tunnel Velocity of 30 Miles per Hour 


\section{Appendix M - Additional Computational CFD Images}

Three specific Reynolds Number values were studied in a computational analysis in order to provide information on lift coefficient and drag coefficient for the augmented propeller blade airfoil. The model was built in Fluent software and analyzed through AoA ranging from -30 to 30 degrees to coincide with the expected values of incidence angle that the propeller would encounter throughout its rotation. The three specific $R E$ values studied were 25,$495 ; 63,649$; and 127,298; corresponding to specific tunnel speeds during experimentation and based on the propeller chord length. This appendix serves to collect the images of the study together for reference, including streamlines, pressure contours, and velocity magnitudes.

\section{Reynolds Number Study at $R E=25495$}

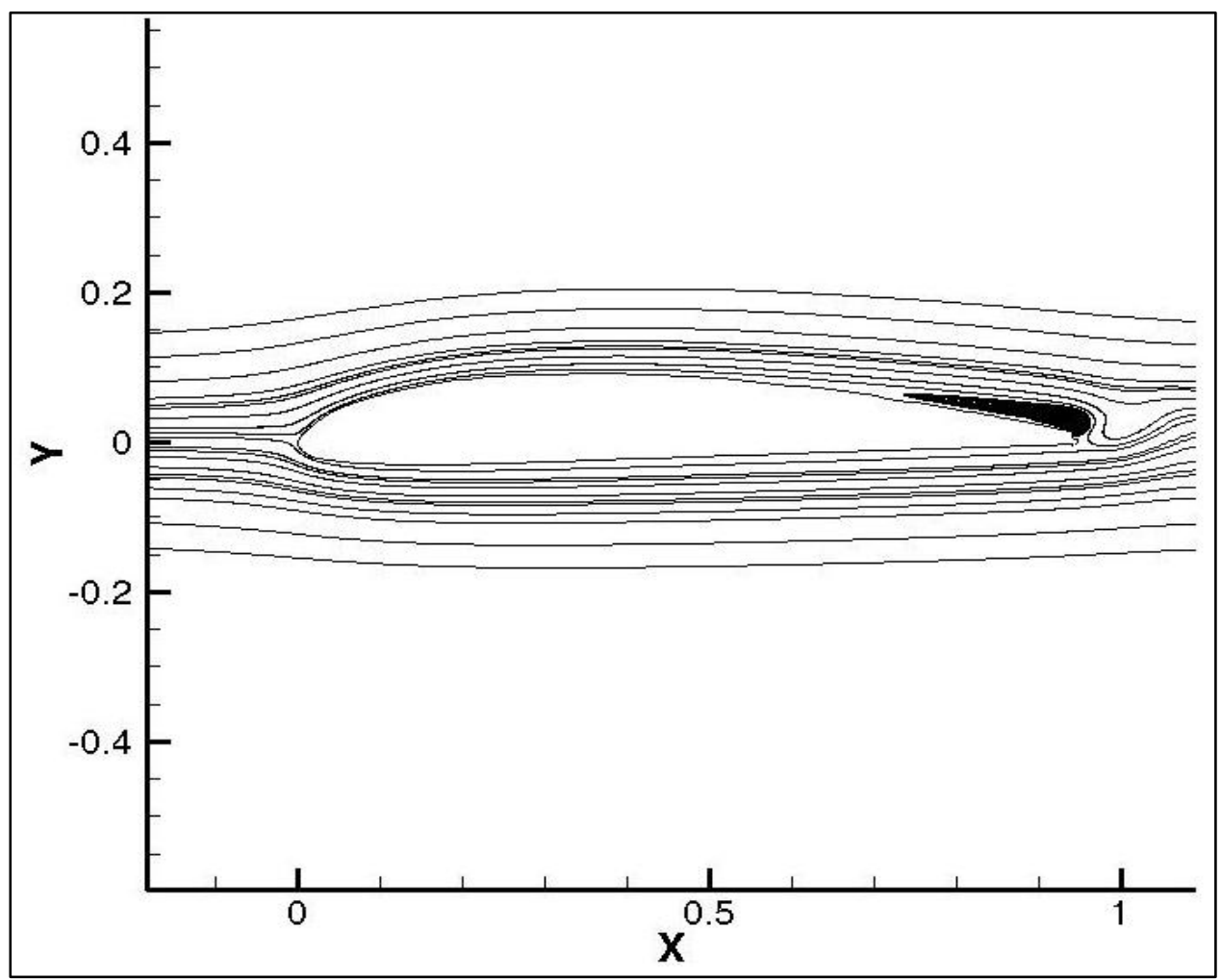

Figure 156. Streamline Plot for $R E=25495(0$ degrees $A o A)$ 


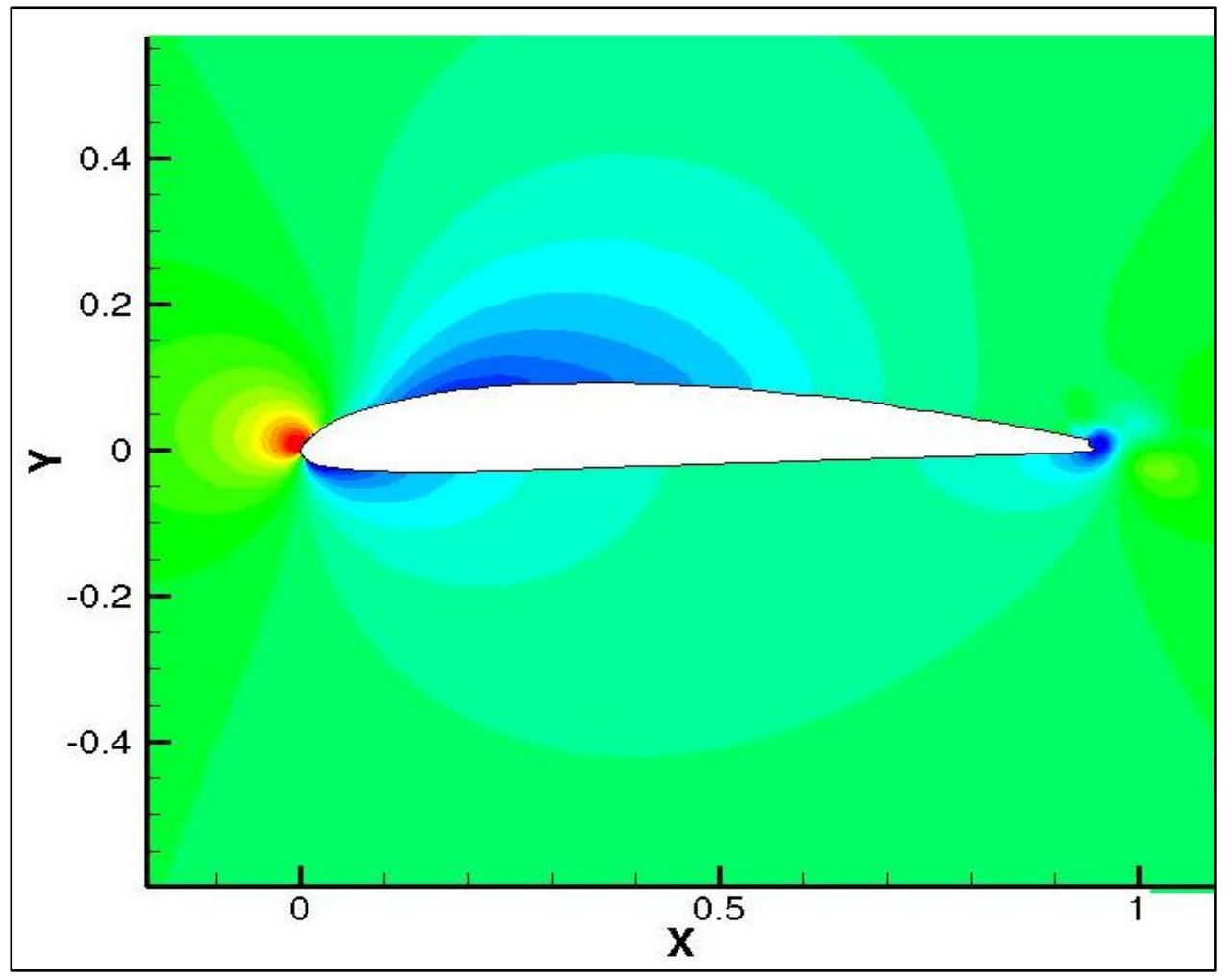

Figure 157. Pressure Contour Plot for $R E=25495(0$ degrees $A o A)$

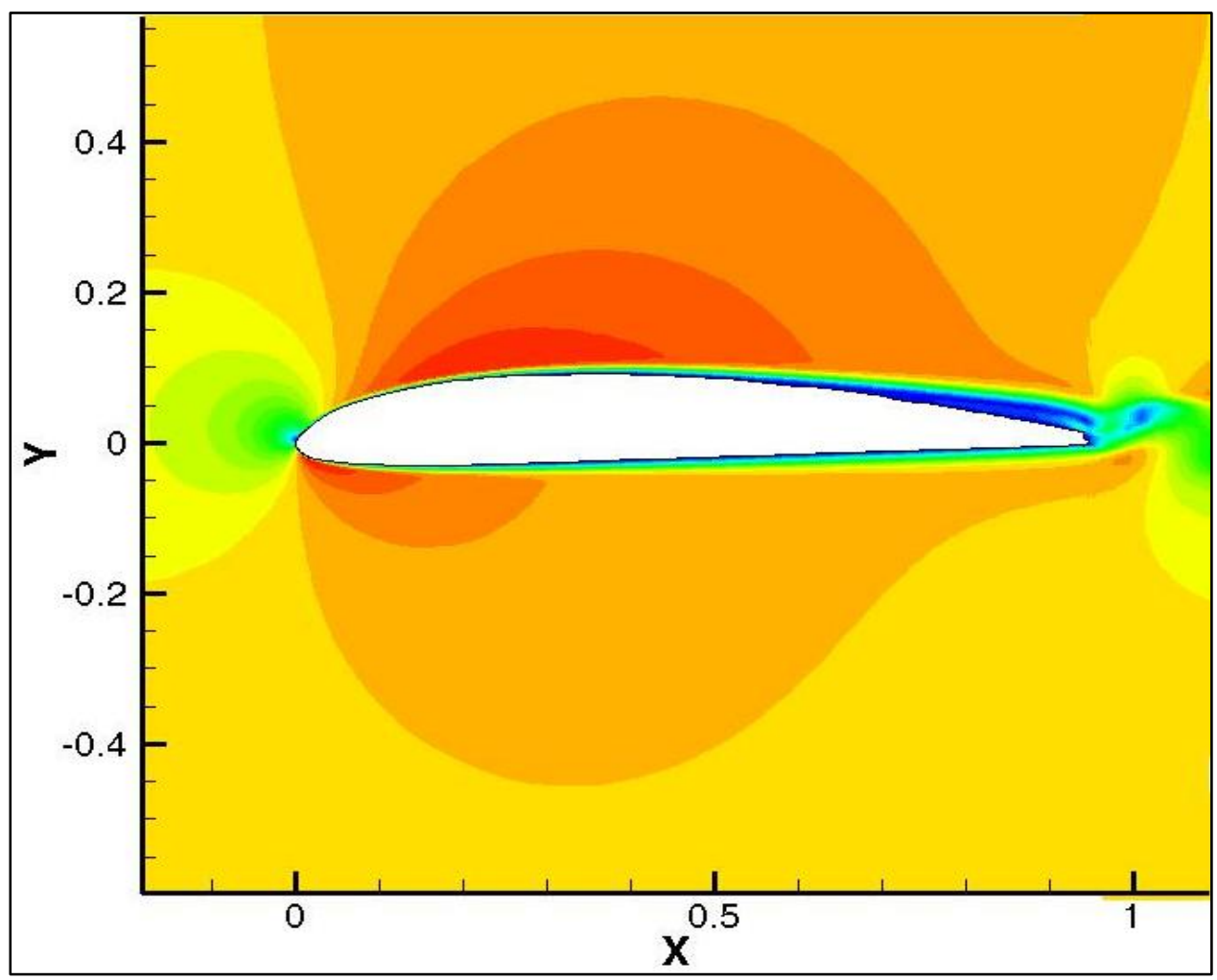

Figure 158. Velocity Magnitude Plot for $R E=25495(0$ degrees $A o A)$ 
Reynolds Number Study at $R E=63649$

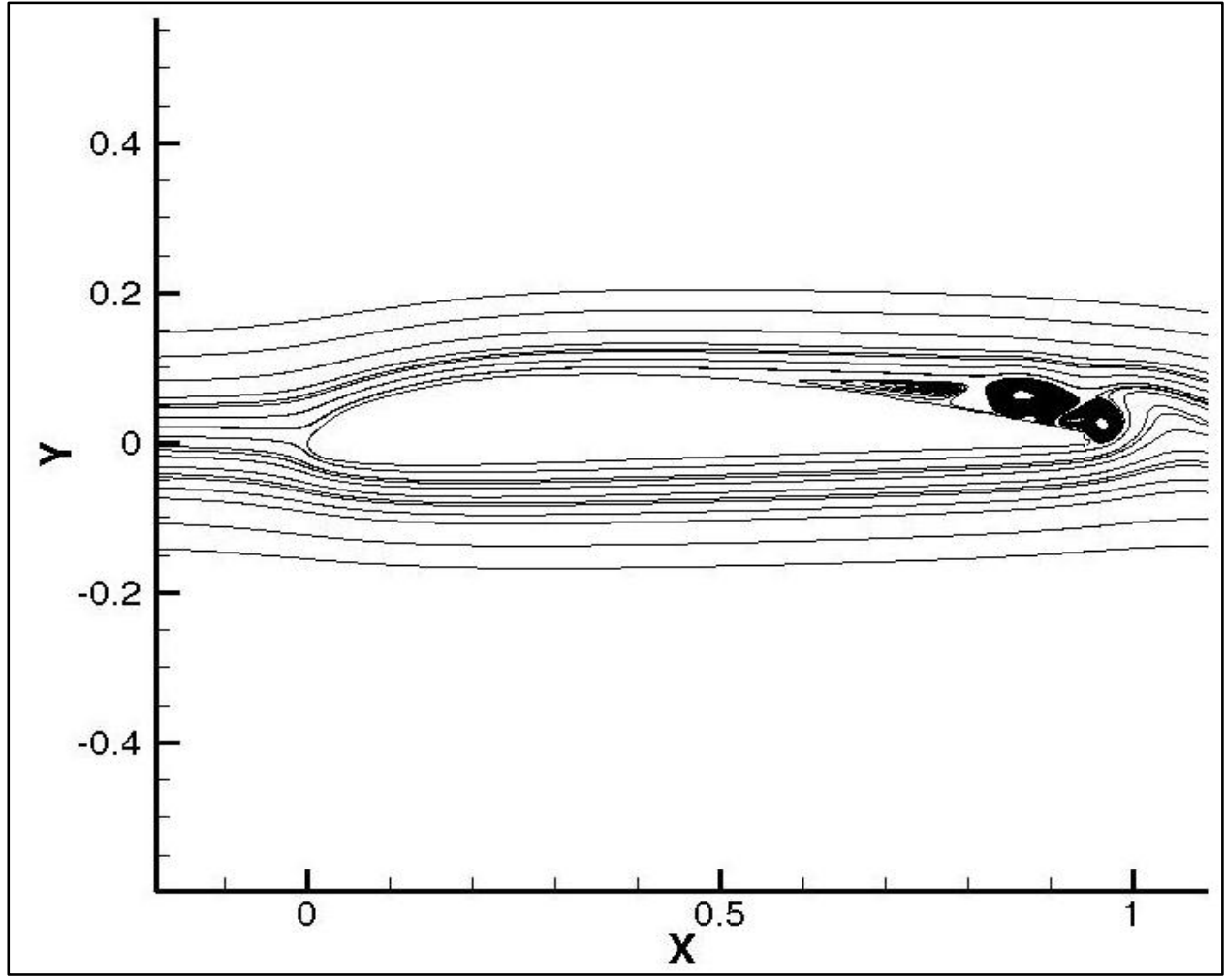

Figure 159. Streamline Plot for $R e=63649(0$ Degrees $A o A)$

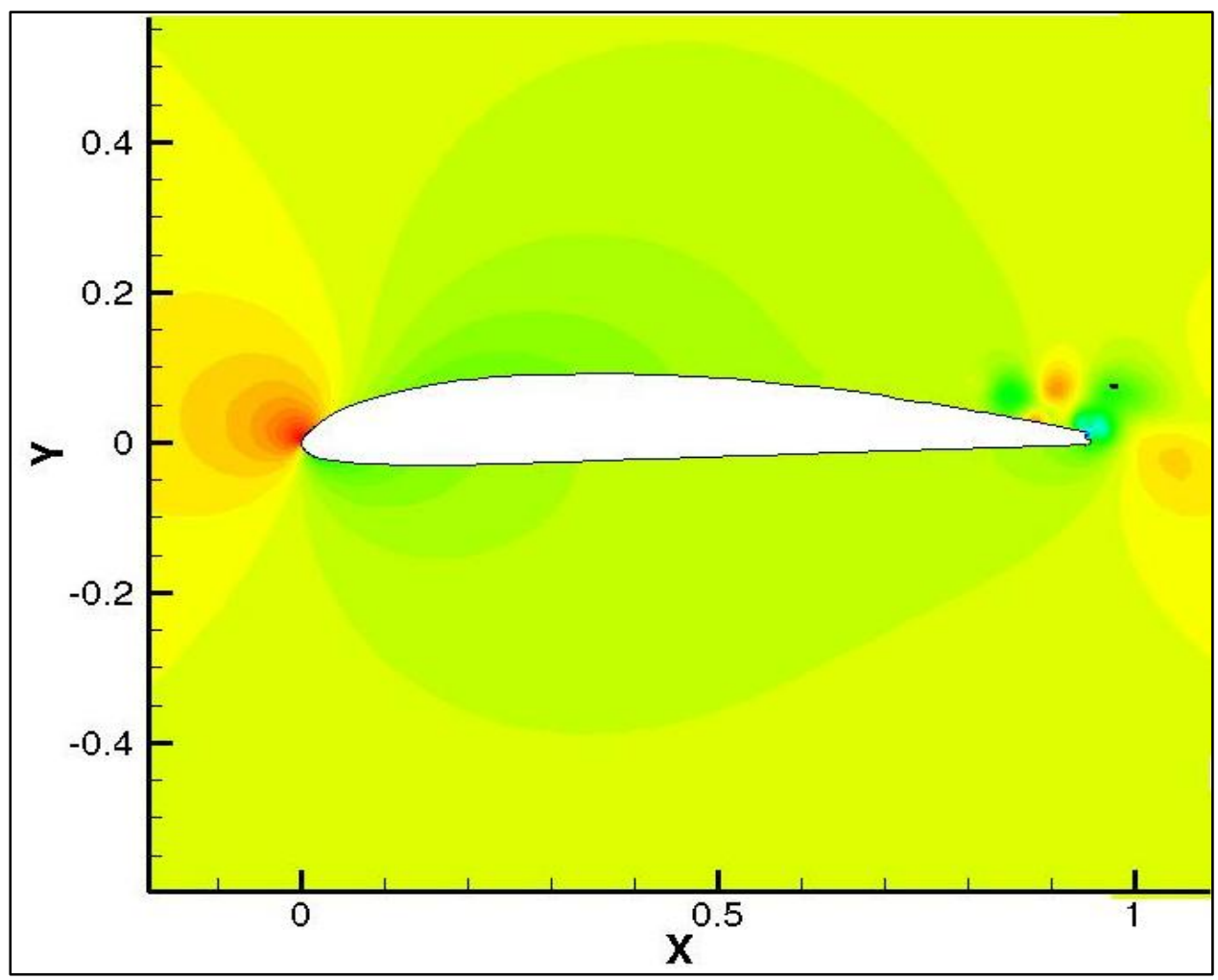

Figure 160. Pressure Contour Plot for $R e=63649(0$ Degrees $A o A)$ 


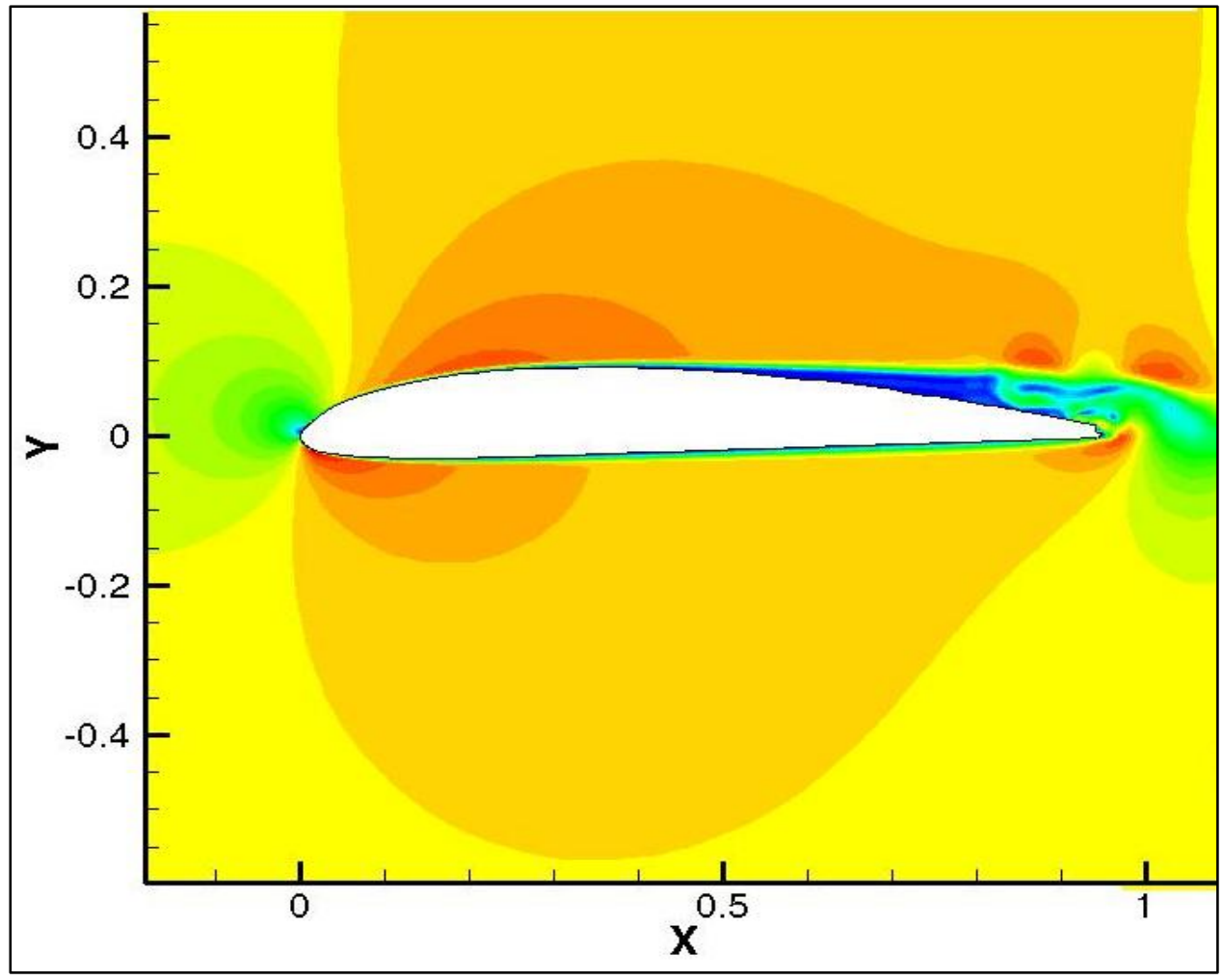

Figure 161. Velocity Magnitude Plot for $R e=63649(0$ Degrees $A o A)$

\section{Reynolds Number Study at $\boldsymbol{R E}=127298$}

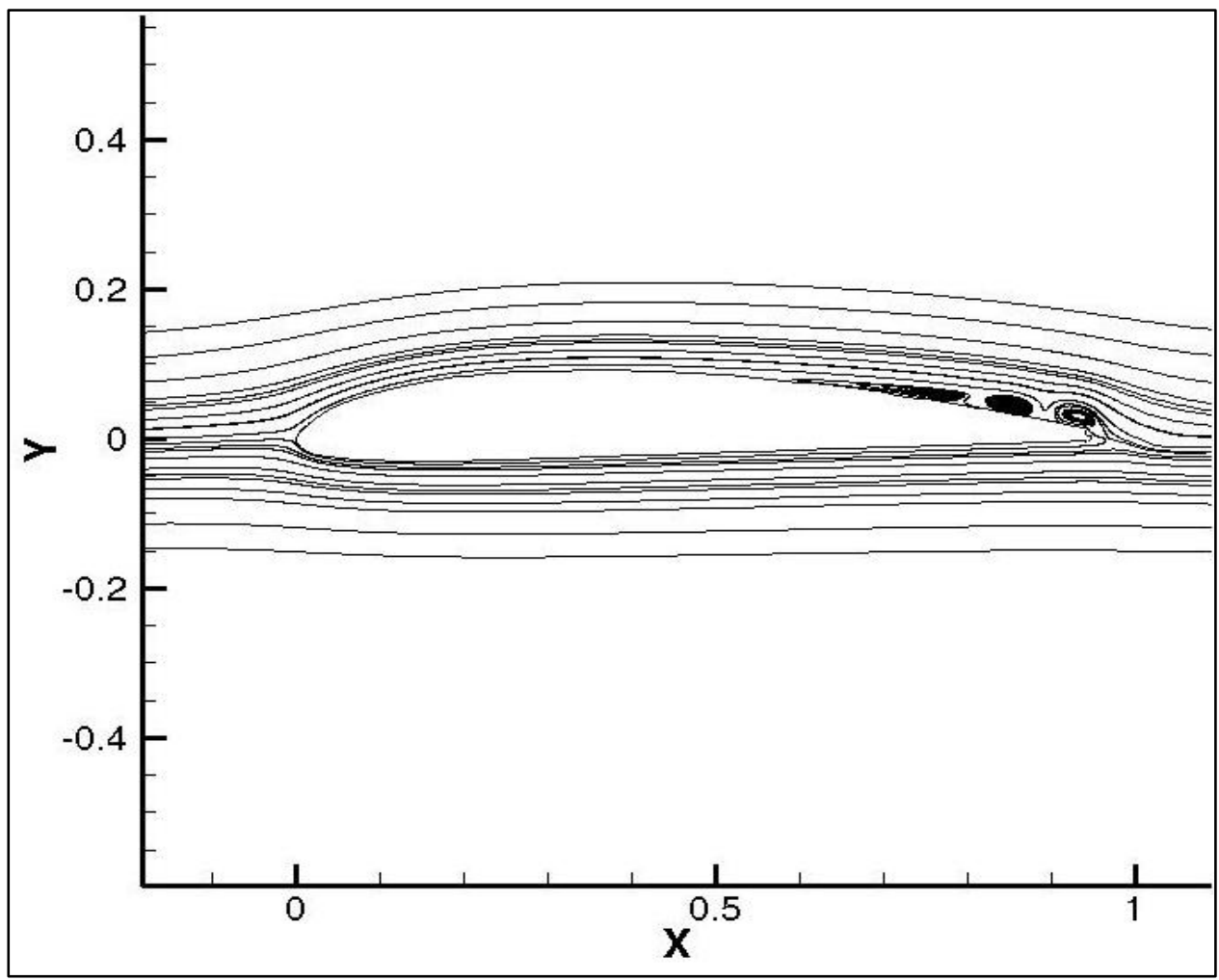

Figure 162. Stream Line Plot at $R E=127298(0$ Degrees $A o A)$ 


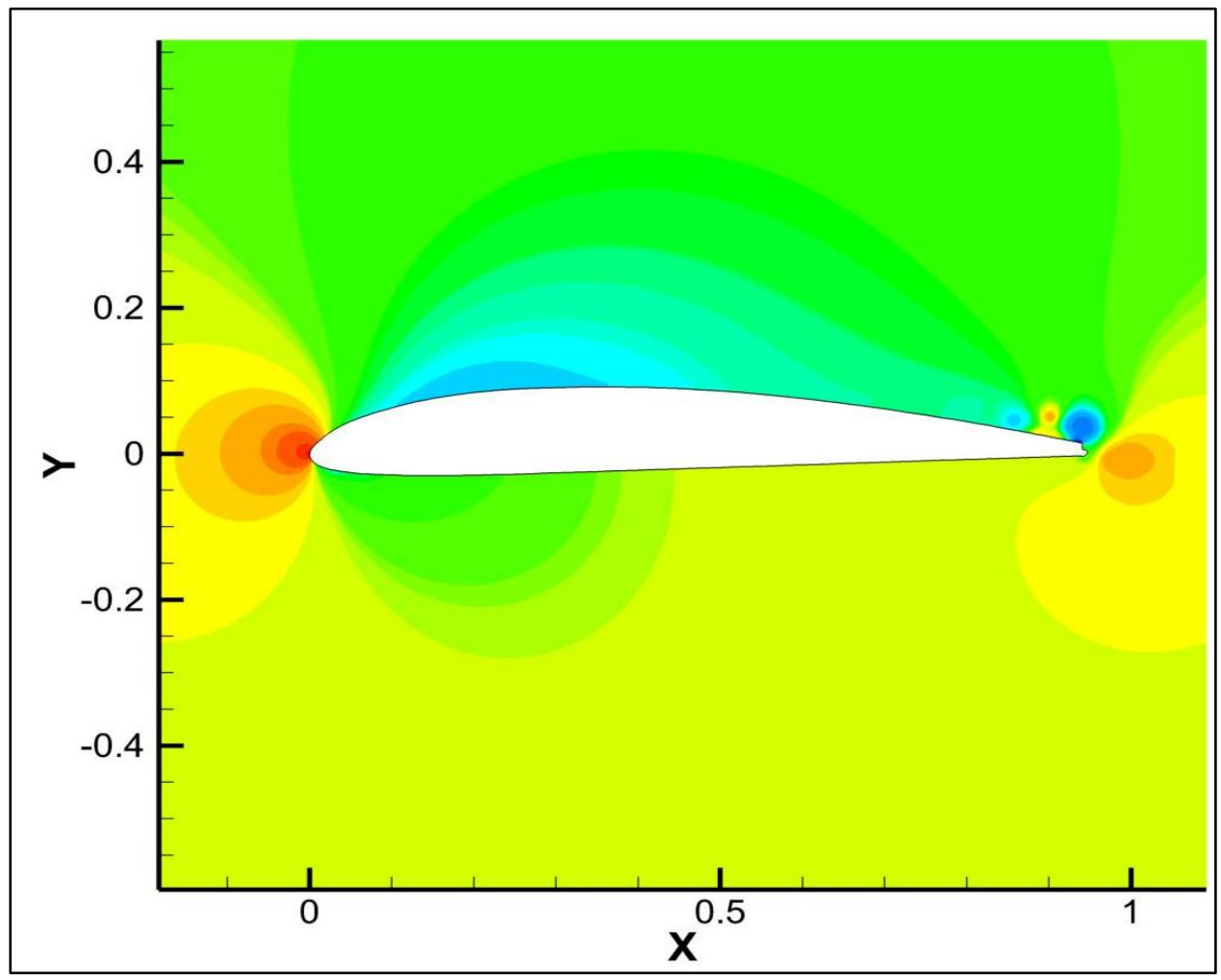

Figure 163. Pressure Contour Plot at $R E=127298(0$ Degrees $A o A)$

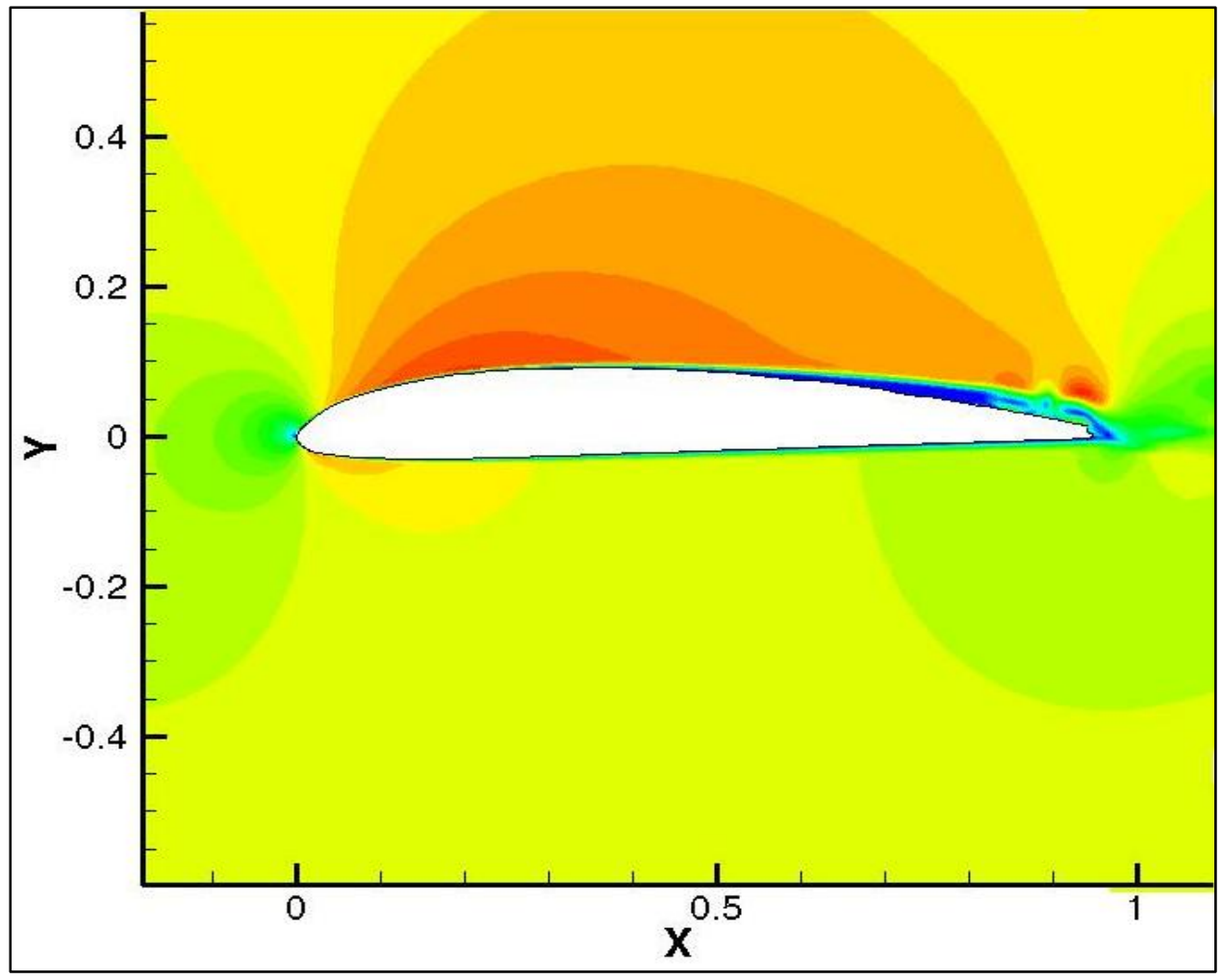

Figure 164. Velocity Magnitude Plot at $R E=127298(0$ Degrees $A o A)$ 


\title{
Augmented Propeller Design with the use of a Passive Circulation Control Pressurization System
}

\author{
Jonathan Kweder
}

\author{
A Dissertation Submitted to the \\ Benjamin M. Statler College of Engineering and Mineral Resources \\ at West Virginia University \\ in Partial Fulfillment of the Requirements \\ for the Degree of
}

Doctorate of Philosophy

in

Mechanical Engineering

Department of Mechanical and Aerospace Engineering

APPROVAL OF THE EXAMINING COMMITTEE

MaryAnn Clarke, Ph.D.

Andrew C. Nix, Ph.D.

Kenneth H. Means, Ph.D

William S. Wayne, Ph.D.

Date

James E. Smith, Ph.D., Chairperson 